\title{
Improved AIOMFAC model parameterisation of the temperature dependence of activity coefficients for aqueous organic mixtures
}

\author{
G. Ganbavale ${ }^{1}$, A. Zuend ${ }^{1,2,3}$, C. Marcolli ${ }^{1,4}$, and T. Peter ${ }^{1}$ \\ ${ }^{1}$ Institute for Atmospheric and Climate Science, ETH Zurich, Zurich, Switzerland \\ ${ }^{2}$ Department of Chemical Engineering, California Institute of Technology, Pasadena, California, USA \\ ${ }^{3}$ Department of Atmospheric and Oceanic Sciences, McGill University, Montreal, Québec, Canada \\ ${ }^{4}$ Marcolli Chemistry and Physics Consulting GmbH, Zurich, Switzerland \\ Correspondence to: A. Zuend (andreas.zuend@ mcgill.ca)
}

Received: 2 June 2014 - Published in Atmos. Chem. Phys. Discuss.: 26 June 2014

Revised: 13 October 2014 - Accepted: 17 October 2014 - Published: 14 January 2015

\begin{abstract}
This study presents a new, improved parameterisation of the temperature dependence of activity coefficients in the AIOMFAC (Aerosol Inorganic-Organic Mixtures Functional groups Activity Coefficients) model applicable for aqueous as well as water-free organic solutions. For electrolyte-free organic and organic-water mixtures the AIOMFAC model uses a group-contribution approach based on UNIFAC (UNIversal quasi-chemical Functional-group Activity Coefficients). This group-contribution approach explicitly accounts for interactions among organic functional groups and between organic functional groups and water. The previous AIOMFAC version uses a simple parameterisation of the temperature dependence of activity coefficients, aimed to be applicable in the temperature range from $\sim 275$ to $\sim 400 \mathrm{~K}$. With the goal to improve the description of a wide variety of organic compounds found in atmospheric aerosols, we extend the AIOMFAC parameterisation for the functional groups carboxyl, hydroxyl, ketone, aldehyde, ether, ester, alkyl, aromatic carbon-alcohol, and aromatic hydrocarbon to atmospherically relevant low temperatures. To this end we introduce a new parameterisation for the temperature dependence. The improved temperature dependence parameterisation is derived from classical thermodynamic theory by describing effects from changes in molar enthalpy and heat capacity of a multi-component system. Thermodynamic equilibrium data of aqueous organic and water-free organic mixtures from the literature are carefully assessed and complemented with new measurements to establish a comprehensive database, covering a wide temperature range ( $\sim 190$ to $\sim 440 \mathrm{~K}$ ) for many of the functional group com-
\end{abstract}

binations considered. Different experimental data types and their processing for the estimation of AIOMFAC model parameters are discussed. The new AIOMFAC parameterisation for the temperature dependence of activity coefficients from low to high temperatures shows an overall improvement of $28 \%$ in comparison to the previous model version, when both versions are compared to our database of experimentally determined activity coefficients and related thermodynamic data. When comparing the previous and new AIOMFAC model parameterisations to the subsets of experimental data with all temperatures below $274 \mathrm{~K}$ or all temperatures above $322 \mathrm{~K}$ (i.e. outside a $25 \mathrm{~K}$ margin of the reference temperature of $298 \mathrm{~K}$ ), applying the new parameterisation leads to $37 \%$ improvement in each of the two temperature ranges considered. The new parameterisation of AIOMFAC agrees well with a large number of experimental data sets. Larger model-measurement discrepancies were found particularly for some of the systems containing multi-functional organic compounds. The affected systems were typically also poorly represented at room temperature and further improvements will be necessary to achieve better performance of AIOMFAC in these cases (assuming the experimental data are reliable). The performance of the AIOMFAC parameterisation is typically better for systems containing relatively small organic compounds and larger deviations may occur in mixtures where molecules of high structural complexity such as highly oxygenated compounds or molecules of high molecular mass (e.g. oligomers) prevail. Nevertheless, the new parameterisation enables the calculation of activity coefficients for a wide variety of different aqueous/water-free organic 
solutions down to the low temperatures present in the upper troposphere.

\section{Introduction}

Atmospheric aerosols are complex mixtures of inorganic and organic components. A large variety of organic compounds account for a significant fraction of the tropospheric aerosol composition. Airborne and ground-based measurements suggest that the aerosols in the free troposphere are composed of $\sim 30 \%$ up to about $\sim 80 \%$ of carbonaceous material mostly in the form of organics (Murphy et al., 2006; Jacobson et al., 2000; Hallquist et al., 2009). Aerosol loading, size distribution, composition, morphology and physical states of particles affect the Earth's radiative budget through the direct effects of aerosols on climate and the indirect effects, in which aerosols act as cloud condensation (CCN) or ice nuclei (IN), affecting cloud particle number concentrations, precipitation, cloud albedo, and lifetime (Lohmann et al., 2005). Organic aerosols are expected to stay in a liquid, viscous semi-solid, or amorphous solid state, since the very large number of organic compounds depresses the temperature at which organic crystal formation takes place (Marcolli et al., 2004; Virtanen et al., 2010; Koop et al., 2011).

Non-ideal interactions between different organic and inorganic species in the particle phase influence water uptake and release (hygroscopicity), may induce liquid-liquid phase separation (LLPS) (e.g. Marcolli and Krieger, 2006; Zuend et al., 2010; Song et al., 2012), influence gas-particle partitioning of semivolatile compounds (e.g. Zuend et al., 2010; Zuend and Seinfeld, 2012), and alter efflorescence and deliquescence relative humidities (e.g. Krieger et al., 2012). Thermodynamic phase equilibrium calculations allow to determine whether the aerosol phase is a liquid (here liquid also refers to a homogeneous, yet potentially highly viscous amorphous phase), a crystalline solid, or a mixture of solid and liquid phases (when assumption of equilibrium is appropriate) and to what degree semivolatile species partition to the condensed phases (Pankow, 2003; Zuend et al., 2010; Zuend and Seinfeld, 2012; Shiraiwa et al., 2013). Furthermore, if the formation of crystalline phases is ignored intentionally in such calculations, metastable equilibria between the gas phase and supersaturated liquid solutions can be predicted. Phase equilibria calculations can be carried out by using composition dependent activity coefficients which account for the non-ideality of the liquid/amorphous phase (Gmehling, 1995; Raatikainen and Laaksonen, 2005; Zuend et al., 2010). The mole fraction based activity coefficient, $\gamma_{s}^{(x)}$ and activity $a_{s}^{(x)}$ of a compound $s$ are related by $a_{s}^{(x)}=$ $\gamma_{s}^{(x)} x_{s}$, where $x_{s}$ is the mole fraction of $s$ in the liquid (homogeneous, amorphous) mixture.

Thermodynamic models for mixtures of organics and water in condensed phases are usually based on the UNIQUAC
(UNIversal QUAsi Chemical) model (Abrams and Prausnitz, 1975) or its group contribution version UNIFAC (UNIquac Functional group Activity Coefficients) (Fredenslund et al., 1975). The original UNIFAC model was developed for vapour-liquid equilibria (VLE) calculations within a temperature range from $\sim 275$ to $\sim 400 \mathrm{~K}$. Using the UNIFAC model outside of its intended temperature range may result in poor predictions of real phase behaviour (Lohmann et al., 2001). For very dilute mixtures, UNIFAC thermodynamic model calculations for component activity coefficients at infinite dilution are sometimes not in agreement with the experimental data. This can be understood since most VLE measurements were performed for liquid mole fractions between 0.02 to 0.98 and, hence, do not provide specific information for the highly dilute regions (Compernolle and Müller, 2014). Inaccurate results were obtained for other types of thermodynamic data, e.g. molar enthalpies of mixing $\left(h^{E}\right)$ or solid-liquid equilibrium (SLE) data. Following the Gibbs-Helmholtz relation, this leads to inaccurate description of activity coefficients as a function of temperature (Lohmann et al., 2001; Gmehling, 2003, 2009). With the original UNIFAC model, due to data insufficiency, inaccurate predictions were often obtained for asymmetric systems (systems containing molecules of different sizes and shapes) (Lohmann et al., 2001; Gmehling, 2003). Since then, the original UNIFAC model has been improved and in addition, modified UNIFAC versions such as modified UNIFAC (Dortmund) and modified UNIFAC (Lyngby) have been developed (Larsen et al., 1987; Hansen et al., 1991; Gmehling et al., 1998, 2002; Jakob et al., 2006), which amended some of the original weaknesses. For mixtures containing multifunctional components, both UNIFAC and modified UNIFAC (Dortmund) sometimes show poor results since the functional group interaction parameters were mainly determined based on experimental data of mixtures of simple, monofunctional components (Weidlich and Gmehling, 1987; Gmehling et al., 2012).

One of the important differences between the UNIFAC model by Hansen et al. (1991), which we call here "standard UNIFAC", and the modified UNIFAC (Dortmund), is the use of a more elaborate parameterisation for the temperature dependence of activity coefficients in the modified UNIFAC (Dortmund) model. However, the modified UNIFAC models still may not provide reliable predictions of activity coefficients at low temperatures relevant in the troposphere. Calculations of water activity $\left(a_{\mathrm{w}}\right)$ of atmospherically relevant aqueous organic solutions have shown that the performance of standard UNIFAC may be poor when the organic fraction consists of multi-functional molecules typically carrying several strong polar functional groups with enhanced hydrogen-bonding potential (Saxena and Hildemann, 1997; Peng et al., 2001). Marcolli and Peter (2005) have therefore proposed improved sets of interaction parameters for standard UNIFAC for alcohols and polyols. Peng et al. (2001) re-parameterised the interaction of the water (group) with the 
carboxyl group and the hydroxyl group based on measured water activities of aqueous systems containing dicarboxylic acids and substituted dicarboxylic and tricarboxylic acids.

For atmospheric applications, an accurate description of aqueous organic mixtures at atmospherically relevant temperatures is required. At low temperatures $a_{\mathrm{w}}$ is a crucial parameter for homogeneous ice nucleation (Koop et al., 2000). Extrapolations of $a_{\mathrm{w}}$ of different aqueous organic solutions measured in the temperature range from the ice melting curve to $313 \mathrm{~K}$ suggest that if the temperature dependence of the activity coefficients is neglected, errors on the order of 10 to $15 \%$ result for $a_{\mathrm{w}}$ at the homogeneous freezing temperature (Zobrist et al., 2008). The uncertainty in predicted homogeneous ice nucleation temperatures is stated as \pm 0.025 in $a_{\mathrm{w}}$ (absolute uncertainties in $a_{\mathrm{w}}$ ) in case of most of the data at higher temperature and \pm 0.05 in $a_{\mathrm{w}}$ for all data collected at ice freezing temperatures (Koop et al., 2000; Koop, 2004). A small uncertainty in $a_{\mathrm{w}}$ of about 0.025 can change the corresponding homogeneous nucleation rate coefficients by 6 orders of magnitude and may significantly affect predictions of the onset of ice crystal formation in cloud microphysical models (Knopf and Rigg, 2011; Alpert et al., 2011). This shows the need for an improved UNIFAC (and AIOMFAC) parameterisation at low temperatures. In addition, the new AIOMFAC parameterisation introduced in this work leads also to substantial improvements in activity coefficient calculations at temperatures significantly higher than room temperature, which is of interest for applications in other fields of science and engineering, such as distillation.

\section{AIOMFAC model}

The AIOMFAC model (Aerosol Inorganic-Organic Mixtures Functional groups Activity Coefficients) by Zuend et al. $(2008,2011)$ is a thermodynamic group-contribution model specifically developed to meet the requirements of typical tropospheric aerosol compositions. The model enables calculations of activity coefficients covering inorganic (water, electrolytes), organic, and organic-inorganic interactions in multi-component solutions over a wide concentration range. AIOMFAC is based on the group-contribution model LIFAC by Yan et al. (1999) and, therefore, includes the standard UNIFAC model, yet also includes the modified parameter sets from Peng et al. (2001) and those from Marcolli and Peter (2005). In its short-range interaction part, the AIOMFAC model shares the simple temperature dependence expressions of the original UNIFAC model and involves only one main group interaction term involving two adjustable parameters, $a_{m, n}$ and $a_{n, m}$ per binary interaction (of groups $m$ and $n$ ). Throughout this article, we will refer to this (original) AIOMFAC model as "AIOMFAC-P1". The aim of this study is to improve the performance of AIOMFAC at low temperatures for multi-component organic + water systems. We will refer to the new AIOMFAC version, with an im- proved temperature dependence parameterisation with two additional main group interaction terms, as AIOMFAC-P3, indicating a three-term parameterisation in the short-range (modified UNIFAC) part. The focus is on a list of major organic functional groups that have been identified in tropospheric aerosols, namely hydroxyl, carboxyl, ketone, ether, ester, aldehyde, alkyl, and aromatic functionalities. Given the focus on organic + water systems, this work does not address the temperature dependence of interaction terms related to inorganic electrolyte/ionic components included in AIOMFAC. A few organic functional groups that have been considered explicitly in the AIOMFAC model development in the past are not included in this work. The excluded organic functionalities are the following: hydroperoxide, peroxy acid peroxide, peroxyacyl nitrate, and organonitrate, all introduced in the AIOMFAC model by Zuend and Seinfeld (2012) based on work by Compernolle et al. (2009). Note that these functional groups are available in AIOMFAC, but on the basis of the AIOMFAC-P1 model parameterisation only.

The thermodynamic group-contribution model AIOMFAC allows thermodynamically consistent calculations of activity coefficients at temperatures close to $298 \mathrm{~K}$ and covers multicomponent solutions containing water, inorganic ions, and organic compounds. For electrolyte-free systems of organic compounds and water, the applicable temperature range is $\sim 275$ to $\sim 400 \mathrm{~K}$, as for the original UNIFAC model. An estimate for the appropriate temperature range of AIOMFAC, when in addition to organic compounds and water also dissolved inorganic ions are included, is $298 \pm 10 \mathrm{~K}$. However, due to a rather weak temperature dependence of activity coefficients in aqueous electrolyte solutions, for many mixtures, the AIOMFAC model may also be applicable in a wider temperature range to good approximation (also relative to other uncertainties associated with a group-contribution model prediction). As mentioned above, the concept of AIOMFAC is based on the LIFAC model (Yan et al., 1999), which merges a Pitzer-like approach with a slightly modified version of the original UNIFAC model to calculate activity coefficients.

The non-ideality of a thermodynamic system is characterised by the excess Gibbs energy $G^{\mathrm{ex}}\left(p, T, n_{j}\right)$, which in AIOMFAC is expressed as the sum of long range (LR), middle range (MR) and short range (SR) contributions:

$G^{\mathrm{ex}}\left(p, T, n_{j}\right)=G_{\mathrm{LR}}^{\mathrm{ex}}+G_{\mathrm{MR}}^{\mathrm{ex}}+G_{\mathrm{SR}}^{\mathrm{ex}}$.

Here, $p$ is the total pressure, $T$ the absolute temperature, and $n_{j}(j=1, \ldots, k)$ the molar amounts of the $k$ components in a system. Mole fraction based activity coefficients $\gamma_{j}^{(x)}$ with $n_{j}$ moles in a mixture are derived from expressions for the different parts of $G^{\mathrm{ex}}$ using the relation

$\ln \gamma_{j}^{(x)}=\left[\frac{\partial G^{\mathrm{ex}} /(R T)}{\partial n_{j}}\right]_{p, T, n_{j^{\prime} \neq j}}$, 
where $R$ is the universal gas constant. Activity coefficients are calculated from the three model parts related to Eq. (1):

$\ln \gamma_{j}^{(x)}=\ln \gamma_{j}^{(x), \mathrm{LR}}+\ln \gamma_{j}^{(x), \mathrm{MR}}+\ln \gamma_{j}^{(x), \mathrm{SR}}$.

Electrolyte solutions, which may range from dilute to highly supersaturated concentrations are, aside from their SR contribution, considered in the Pitzer-like part, which combines LR and MR interactions. The LR interactions are described by an extended Debye-Hückel term and represents contributions by Coulomb electrostatic forces between permanently charged ions, moderated by the presence of the dielectric solvent medium (e.g. a homogeneous mixture of water + organic compounds act as the solvent medium). The MR part represents the effects of interactions involving ions and permanent or induced dipoles and contains most of the adjustable parameters to describe concentrated aqueous electrolyte solutions and organic-inorganic mixtures. The original AIOMFAC model by Zuend et al. (2008) has been extended and re-parameterised to include organic-inorganic interactions of most of the functional groups typically present in atmospheric organic compounds (carboxyl, hydroxyl, ketone, aldehyde, ether, ester, alkyl, aromatic carbon-alcohol, and aromatic hydrocarbon) (Zuend et al., 2011). In addition, based on the approach and UNIFAC parameters determined by Compernolle et al. (2009), Zuend and Seinfeld (2012) introduced in AIOMFAC the functional groups hydroperoxide, peroxy acid, and peroxide, including estimated interaction parameters with the inorganic ions of the model. For further details of the thermodynamic description of the LR and MR interactions within the Pitzer-like part of AIOMFAC we refer to Zuend et al. $(2008,2011)$. The interactions among noncharged species (organic molecules and water) are calculated in the SR part of AIOMFAC, see Sect. 2.2.

\subsection{Group-contribution method}

A group-contribution concept similar to the one in UNIFAC has been adopted for the AIOMFAC model. According to the group-contribution concept, it is assumed that the system (and its organic constituents) are composed of combinations of functional groups instead of whole molecule entities. The advantage of applying the group-contribution method is that a very large number of organic compounds can be defined using the various combinations of a limited number of functional groups. In accordance to the UNIFAC model, the functional groups are further classified into so-called main groups and subgroups for their application in different model parts (Fredenslund et al., 1975; Marcolli and Peter, 2005; Zuend et al., 2008, 2011). The main groups cover subgroups of the same functionality that only differ by the number of hydrogen atoms. The subgroup classification of a variety of organic compounds can be found in Table 1 .

\subsection{Short-range contribution}

As in the UNIFAC model, in the SR part of AIOMFAC, activity coefficients of a mixture component $j$ are in general expressed as the sum of contributions of a combinatorial part (denoted by superscript $\mathrm{C}$ ), which accounts for the size and shape of the molecule, and the residual part (denoted by superscript $\mathrm{R}$ ), which reflects the residual contribution from intermolecular (inter-group) interactions (Fredenslund et al., 1975; Marcolli and Peter, 2005; Zuend et al., 2008).

$\ln \gamma_{j}^{\mathrm{SR},(x)}=\ln \gamma_{j}^{\mathrm{C}}+\ln \gamma_{j}^{\mathrm{R}}$

The expression for the combinatorial part of UNIFAC is (Fredenslund et al., 1975; Zuend et al., 2008):

$\ln \gamma_{j}^{\mathrm{C}}=\ln \frac{\Phi_{j}}{x_{j}}+\frac{z}{2} q_{j} \ln \frac{\Theta_{j}}{\Phi_{j}}+l_{j}-\frac{\Phi_{j}}{x_{j}} \sum_{j^{\prime}} x_{j^{\prime}} l_{j^{\prime}}$

where

$\Phi_{j}=\frac{r_{j} x_{j}}{\sum_{j^{\prime}} r_{j^{\prime}} x_{j^{\prime}}} ; \quad \Theta_{j}=\frac{q_{j} x_{j}}{\sum_{j^{\prime}} q_{j^{\prime}} x_{j^{\prime}}}$

and

$r_{j}=\sum_{t} v_{t}^{(j)} R_{t} ; \quad q_{j}=\sum_{t} v_{t}^{(j)} Q_{t} ;$

$l_{j}=\frac{z}{2}\left(r_{j}-q_{j}\right)-\left(r_{j}-1\right)$.

In these equations, $x_{j}$ is the mole fraction of component $j$, and $v_{t}^{(j)}$ denotes the number of subgroups of type $t$ present in a formula unit of component $j$. The relative van der Waals subgroup volume and surface area are given by $R_{t}$ and $Q_{t}$, respectively. The lattice coordinate number $z$ is typically assumed to be a constant set to $z=10$ (Fredenslund et al., 1975). Relative subgroup volume and surface area parameters published by Hansen et al. (1991) are used for the neutral species.

Enthalpic interaction contributions are considered in the residual part of UNIFAC. The residual part $\left(\gamma_{j}^{\mathrm{R}}\right)$ of the activity coefficient of component $j$ is given by the following:

$\ln \gamma_{j}^{\mathrm{R}}=\sum_{t} v_{t}^{(j)}\left[\ln \Gamma_{t}-\ln \Gamma_{t}^{(j)}\right]$,

where $\Gamma_{t}$ is the group residual activity coefficient in the mixture, while $\Gamma_{t}^{(j)}$ represents the one in a reference liquid containing only compound $j . v_{t}^{(j)}$ is the number of subgroups of type $t$ in molecule $j$. The residual activity coefficient of subgroup $t$ is as follows:

$\ln \Gamma_{t}=Q_{t}\left[1-\ln \left(\sum_{m} \Theta_{m} \Psi_{m, t}\right)-\sum_{m}\left(\frac{\Theta_{m} \Psi_{t, m}}{\sum_{n} \Theta_{n} \Psi_{n, m}}\right)\right]$, 
where

$\Theta_{m}=\frac{Q_{m} X_{m}}{\sum_{n} Q_{n} X_{n}}$

In these expressions $\Theta_{m}$ is the relative surface area fraction of subgroup $m, X_{m}$ is the mole fraction of $m$ in the mixture of subgroups (note: $X_{m}$ is different from the mole fraction $x_{j}$ that would refer to the mixture of components, not mixture of subgroups). The standard UNIFAC temperature-dependent interaction between the subgroups $m$ and $n$ is given by Fredenslund et al. (1975):

$\ln \Psi_{n, m}=-\left[\frac{U_{n, m}-U_{n, n}}{R T}\right]$,

where $U_{n, m}$ is a measure of change in the molar Gibbs free energy due to interaction between subgroups $m$ and $n$. Eq. (12) is typically represented in the more compact form of Eq. (13).

$\ln \Psi_{n, m}=-\left[\frac{a_{n, m}}{T}\right]$

Due to the formulation of Eq. (12), with equivalent differences for the interactions between subgroups $m$ and $n$ (with the difference $U_{m, n}-U_{m, m}$ ), the main group interaction parameters $a_{n, m}$ are unsymmetrical, i.e. $a_{n, m} \neq a_{m, n}$. Note that all interaction parameters are only resolved on the main group level, i.e. all subgroups of a certain main group interacting with a subgroup of a different main group will share the same interaction parameter. Hence, we refer to the set of $a_{n, m}$ as main group interaction parameters. In standard UNIFAC, the $a_{n, m}$ interaction parameters of organic solutions were estimated using a large database of experimental vapour-liquid equilibrium (VLE) and a few liquid-liquid equilibrium (LLE) data sets. This approach leads to satisfying predictions for vapour-liquid equilibria, but reliable simultaneous description of VLE, LLE, solid-liquid equilibria (SLE), and molar enthalpies of mixing $\left(h^{E}\right)$ can often not be obtained (Lohmann et al., 2001). In order to overcome these deficiencies of the standard UNIFAC, modified UNIFAC (Dortmund) uses three main group interaction parameters in the residual part to improve predictions of activity coefficients over a wider range of temperatures and different types of phase equilibria (Gmehling et al., 1993; Lohmann et al., 2001; Jakob et al., 2006):

$\ln \Psi_{n, m}=-\left[\frac{a_{n, m}+b_{n, m} T+c_{n, m} T^{2}}{T}\right]$.

In modified UNIFAC (Dortmund) the relative van der Waals volume $\left(R_{t}\right)$ and surface $\left(Q_{t}\right)$ coefficients for the structural groups are not calculated from molecular parameters as in the standard UNIFAC approach; rather, they are fit together with the interaction parameters $\left(a_{n, m}, b_{n, m}, c_{n, m}\right)$ to experimental data.
The AIOMFAC model is aimed for a wide range of applications, including the calculation of solid-liquid equilibria and other thermodynamic phase equilibria. The temperature dependence of these equilibria is related to the molecular interaction of the components in the liquid phase. Hence, the temperature dependence of chemical reaction equilibria and phase equilibria are described by the same thermodynamic functions and we can express them with parameterisations for the temperature dependence of reaction equilibria. According to Clarke and Glew (1966), if the equilibrium constant $K_{p}$ of a chemical reaction or exchange process is a function of temperature, the changes in the standard thermodynamic functions, i.e. change in molar Gibbs free energy $\Delta g^{\circ}$, change in molar enthalpy $\Delta h^{\circ}$ and change in molar heat capacity $\Delta c_{p}^{\circ}$ are directly related to $K_{p}$ (by definition) and are well-behaved functions of $T$. The relationship for the equilibrium constant $K_{p}$ and temperature $T$, when excluding higher order derivatives of the molar heat capacity change with temperature, are given by (Clarke and Glew, 1966):

$R \ln K_{p}=-\frac{\Delta g_{T_{\ominus}}^{\circ}}{T_{\ominus}}+\Delta h_{T_{\ominus}}^{\circ}\left[\frac{1}{T_{\ominus}}-\frac{1}{T}\right]+\Delta c_{p, T_{\ominus}}^{\circ}\left[\frac{T_{\ominus}}{T}-1+\ln \frac{T}{T_{\ominus}}\right]$,

where $T_{\ominus}$ is a reference temperature at which the changes in $\Delta g^{\circ}, \Delta h^{\circ}$ and $\Delta c_{p}^{\circ}$ are determined or known. In order to better describe activity coefficients at low (and high) temperatures while preserving compatibility with the already estimated values of the interaction parameters $a_{n, m}$ at room temperature, we introduce a similar but slightly modified expression for $\Psi_{n, m}$. We define the temperature dependent interaction potential in AIOMFAC as

$\ln \Psi_{n, m}=-\frac{a_{n, m}}{T}+b_{n, m}\left[\frac{1}{T_{\ominus}}-\frac{1}{T}\right]+c_{n, m}\left[\frac{T_{\ominus}}{T}-1+\ln \frac{T}{T_{\ominus}}\right]$,

with the reference temperature $T_{\ominus}=298.15 \mathrm{~K}$. The first term on the right hand side is exactly the same as in standard UNIFAC, but slightly different from the equivalent term in Eq. (15), due to the use of actual temperature $T$ instead of reference temperature $T_{\ominus}$ for consistency with standard UNIFAC/AIOMFAC. This term in Eq. (16) therefore includes both changes in $\Delta g_{T_{\ominus}}^{\circ}$ as well as a part of the changes related to $\Delta h_{T_{\ominus}}^{\circ}$ (note: this is obvious when considering a hypothetical, very high reference temperature for the second term on the right hand side of Eq. 16). The second term includes the change in enthalpy and in addition acts as a correction term for parameters $a_{n, m}$ at temperatures different from the reference temperature. The third term accounts for the contribution related to the heat capacity change of a main group interaction, whose importance increases for temperatures far away from the reference temperature.

We use a database of experimental thermodynamic equilibrium data for organic and organic-water systems (see Sects. 3 and 4), covering a wide temperature and concentration range, to determine simultaneously the AIOMFAC 
group interaction parameters $b_{n, m}$ and $c_{n, m}$ for pertaining organic functional groups. To preserve compatibility with the AIOMFAC model version of Zuend et al. (2011), and its fitted organic-inorganic interaction parameters at room temperature, all group-interaction parameters $a_{m, n}$ are kept the same, which implies that the performance of AIOMFAC at $298.15 \mathrm{~K}$ will not be altered by the improved three-parameter temperature-dependence parameterisation. With the goal to describe a wide variety of organic compounds at relevant atmospheric temperatures, we focus on the aqueous systems of oxidised organics at lower temperatures. The temperature dependence formulation given by Eq. (16) will at this point only be parameterised for interactions between the UNIFAC main groups alkyl $\left(\mathrm{CH}_{\mathrm{n}}\right)$, specific variants of alkyl groups in alcohols $\left(\mathrm{CH}_{\mathrm{n}}^{\text {[alc] }}\right),\left(\mathrm{CH}_{\mathrm{n}}^{[\text {alc-tail] }}\right)$, and $\left(\mathrm{CH}_{\mathrm{n}}^{[\mathrm{OH}]}\right)$, hydroxyl $(\mathrm{OH})$, carboxyl $(\mathrm{COOH})$, ketone $\left(\mathrm{CH}_{\mathrm{n}} \mathrm{CO}\right)$, aldehyde $(\mathrm{CHO})$, ether $\left(\mathrm{CH}_{\mathrm{n}} \mathrm{O}\right)$, ester $(\mathrm{CCOO})$, alkenyl $(\mathrm{C}=\mathrm{C})$, aromatic carbon $\left(\mathrm{ACH}_{\mathrm{n}}\right)$, aromatic carbon-alcohol $(\mathrm{ACOH})$ (a phenol group), and water $\left(\mathrm{H}_{2} \mathrm{O}\right)$. For all other group interactions not considered, $b_{n, m}$ and $c_{n, m}$ are set to zero so that Eq. (16) reduces to Eq. (13). The rules for the use of specific alkyl groups are described below. With this approach, an improved description of activities for organic systems at low temperatures can be achieved, while maintaining compatibility with standard UNIFAC, hence, preserving the applicability of AIOMFAC to a wider range of functional groups.

The UNIFAC functional groups in AIOMFAC include some modifications with respect to standard UNIFAC to better describe the specific properties of organic aerosol constituents, which typically are molecules composed of several (polar) functional groups. Therefore a more detailed description of alcohol/polyol group interaction parameters published by Marcolli and Peter (2005) was implemented, where the relative positions of the $\mathrm{OH}$ functional group, as well as those of neighbouring alkyl groups are taken into account (Zuend et al., 2011). According to this approach, water-alkyl and water-hydroxyl group interaction parameters for alcohols/polyols are treated specifically, while keeping the alkyl-hydroxyl interaction parameter unchanged in order to maintain the performance of AIOMFAC in case of water free alkane/alcohol systems compatible with standard UNIFAC. Except for $\mathrm{CH}_{\mathrm{n}}^{[\mathrm{OH}]}$ groups directly bonded to an $\mathrm{OH}$ group, standard UNIFAC $\mathrm{CH}_{\mathrm{n}}$ groups are used for alkyl groups in multi-functional molecules that contain hydroxyl groups combined with different other functional groups. Another difference with respect to standard UNIFAC is that we use the parameters of Peng et al. (2001) for the interaction of the $\mathrm{COOH}$ group with the $\mathrm{OH}$ group and the $\mathrm{H}_{2} \mathrm{O}$ group. The use of these modified UNIFAC group interaction parameters leads to improvements for certain aqueous systems of alcohols, dicarboxylic and hydroxycarboxylic acids, while being compatible with the use of standard UNIFAC parameters for other group interactions, as described in more detail in Zuend et al. (2011).

\section{Experimental data}

A reliable estimation of group interaction parameters and temperature dependence relies on a comprehensive database covering a wide variety of compounds consisting of the targeted functional groups with consideration of a large temperature range. In order to establish such a database, an extensive literature search was carried out. The DETHERM databank (Gesellschaft für Chemische Technik und Biotechnologie e.V., http://www.dechema.de), which offers the world's largest collection of thermodynamic mixture data was used to check the completeness of the literature search and to directly purchase data for which the original publication was not easily accessible.

Figure 1 provides an overview of the database collected in this study. The matrix lists the number of data sets at temperatures substantially different from $298 \mathrm{~K}$ available for each main group pair interaction. The green bars indicate the maximum number of overall data sets including all data types available for each main group interaction pair. For each interaction pair, the highest temperature (red shaded boxes) and lowest temperature (blue shaded boxes), for which data points are available, is indicated. In addition, listed are the median and arithmetic mean values of the assigned initial data set weighting values $\left(w_{d}^{\text {init }}\right)$ pertaining to each main group interaction pair. The combination of these values serves as an approximate measure of the data quality. A higher median value $\left(\operatorname{median}\left(w_{d}^{\text {init }}\right) \geq \sim 1\right)$, paired with a large number of data sets and a wide temperature range covered, indicates the availability of reliable thermodynamic equilibrium data for the model parameterisation. For certain group interactions, the data coverage and reliability is clearly lacking, which was considered in the model parameterisation.

The database overall consists of 677 data sets covering different data types, for monofunctional and multi-functional organic molecules in aqueous and water-free mixtures of binary and ternary systems. Table 1 lists the data sets and the data types used for determining the main group interaction parameters $\left(b_{n, m}\right.$ and $\left.c_{n, m}\right)$ in the SR part of the AIOMFAC model. The table lists the mixture components, main groups, chemical formula (subgroups), data type, number of data points, temperature range, assigned initial weighting used in the model parameter fit, and the data source. Tables reporting new water activity measurements are provided in the Appendix (Tables A1 to A8). Different data types and their processing for use with the model parameterisation are described in the following.

\subsection{Solid-liquid equilibrium data}

Most low temperature data available for the model parameterisation are binary SLE data with water and an organic component. SLE data can be obtained by measuring the melting point depression of solutes as a function of solution 
Table 1. Components, main groups, temperature range, number of data points $\left(N_{d}\right)$, initial weighting ( $\left.w_{d}^{\text {init }}\right)$ and references of "water + organic" and "organic + organic" data sets used (where $w_{d}^{\text {init }}>0$ ) for the short-range parameterisation of organic main group $\leftrightarrow$ water and organic $\leftrightarrow$ organic main group interactions.

\begin{tabular}{|c|c|c|c|c|c|c|c|}
\hline Organic compounds & Org. main groups & Chemical formula (subgroups) & $T(\mathrm{~K})$ & Data type & $N_{d}$ & $w_{d}^{\text {init }}$ & Reference \\
\hline \multicolumn{8}{|c|}{ - water + alcohol/polyol - } \\
\hline ethanol & $\mathrm{CH}_{\mathrm{n}}^{[\text {alc-tail }]}, \mathrm{CH}_{\mathrm{n}}^{[\mathrm{OH}]}, \mathrm{OH}$ & $\left(\mathrm{CH}_{3}^{[\text {alc-tail }]}\right)\left(\mathrm{CH}_{2}^{[\mathrm{OH}]}\right)(\mathrm{OH})$ & $220-269$ & SLE & 7 & 5.00 & Ross (1954) \\
\hline ethanol & $\mathrm{CH}_{\mathrm{n}}^{[\text {alc-tail }]}, \mathrm{CH}_{\mathrm{n}}^{[\mathrm{OH}]}, \mathrm{OH}$ & $\left(\mathrm{CH}_{3}^{[\text {[alc-tail }]}\right)\left(\mathrm{CH}_{2}^{[\mathrm{OH}]}\right)(\mathrm{OH})$ & $265-273$ & SLE & 31 & 5.00 & Knight (1962) \\
\hline ethanol & $\mathrm{CH}_{\mathrm{n}}^{[\text {alc-tail }]}, \mathrm{CH}_{\mathrm{n}}^{[\mathrm{OH}]}, \mathrm{OH}$ & $\left(\mathrm{CH}_{3}^{[\text {[alc-tail }]}\right)\left(\mathrm{CH}_{2}^{[\mathrm{OH}]}\right)(\mathrm{OH})$ & $211-273$ & SLE & 62 & 1.00 & Pickering (1893) \\
\hline ethanol & $\mathrm{CH}_{\mathrm{n}}^{[\text {alc-tail }]}, \mathrm{CH}_{\mathrm{n}}^{[\mathrm{OH}]}, \mathrm{OH}$ & $\left(\mathrm{CH}_{3}^{[\text {[alc-tail }]}\right)\left(\mathrm{CH}_{2}^{[\mathrm{OH}]}\right)(\mathrm{OH})$ & $307-318$ & VLE & 11 & 1.00 & Gmehling and Onken (1977) \\
\hline ethanol & $\mathrm{CH}_{\mathrm{n}}^{[\text {alc-tail }]}, \mathrm{CH}_{\mathrm{n}}^{[\mathrm{OH}]}, \mathrm{OH}$ & $\left(\mathrm{CH}_{3}^{[\text {[alc-tail }]}\right)\left(\mathrm{CH}_{2}^{[\mathrm{OH}]}\right)(\mathrm{OH})$ & $341-353$ & VLE & 11 & 1.00 & Gmehling and Onken (1977) \\
\hline ethanol & $\mathrm{CH}_{\mathrm{n}}^{[\text {[alc-tail }]}, \mathrm{CH}_{\mathrm{n}}^{[\mathrm{OH}]}, \mathrm{OH}$ & $\left(\mathrm{CH}_{3}^{[\text {[alc-tail }]}\right)\left(\mathrm{CH}_{2}^{[\mathrm{OH}]}\right)(\mathrm{OH})$ & $350-363$ & VLE & 11 & 1.00 & Gmehling and Onken (1977) \\
\hline 1-propanol & $\mathrm{CH}_{\mathrm{n}}^{[\text {[alc-tail }]}, \mathrm{CH}_{\mathrm{n}}^{[\mathrm{OH}]}, \mathrm{OH}$ & $\left(\mathrm{CH}_{3}^{[\text {[alc-tail }]}\right)\left(\mathrm{CH}_{2}^{[\text {alc-tail }]}\right)\left(\mathrm{CH}_{2}^{[\mathrm{OH}]}\right)(\mathrm{OH})$ & $263-270$ & SLE & 3 & 5.00 & Ross (1954) \\
\hline 1-propanol & $\mathrm{CH}_{\mathrm{n}}^{[\text {[alc-tail }]}, \mathrm{CH}_{\mathrm{n}}^{[\mathrm{OH}]}, \mathrm{OH}$ & $\left(\mathrm{CH}_{3}^{[\text {[alc-tail }]}\right)\left(\mathrm{CH}_{2}^{[\text {alc-tail] }}\right)\left(\mathrm{CH}_{2}^{[\mathrm{OH}]}\right)(\mathrm{OH})$ & $264-270$ & SLE & 7 & 5.00 & Chapoy et al. (2008) \\
\hline 1-propanol & $\mathrm{CH}_{\mathrm{n}}^{[\text {[alc-tail }]}, \mathrm{CH}_{\mathrm{n}}^{[\mathrm{OH}]}, \mathrm{OH}$ & $\left(\mathrm{CH}_{3}^{[\text {[alc-tail }]}\right)\left(\mathrm{CH}_{2}^{[\text {alc-tail }]}\right)\left(\mathrm{CH}_{2}^{[\mathrm{OH}]}\right)(\mathrm{OH})$ & $264-270$ & SLE & 12 & 5.00 & Pickering (1893) \\
\hline 1-propanol & $\mathrm{CH}_{\mathrm{n}}^{[\text {[alc-tail }]}, \mathrm{CH}_{\mathrm{n}}^{[\mathrm{OH}]}, \mathrm{OH}$ & $\left(\mathrm{CH}_{3}^{[\text {[alc-tail }]}\right)\left(\mathrm{CH}_{2}^{[\text {alc-tail] }}\right)\left(\mathrm{CH}_{2}^{[\mathrm{OH}]}\right)(\mathrm{OH})$ & $264-270$ & SLE & 14 & 5.00 & Pickering (1893) \\
\hline 1-propanol & $\mathrm{CH}_{\mathrm{n}}^{[\text {[alc-tail }]}, \mathrm{CH}_{\mathrm{n}}^{[\mathrm{OH}]}, \mathrm{OH}$ & $\left(\mathrm{CH}_{3}^{[\text {[alc-tail }]}\right)\left(\mathrm{CH}_{2}^{[\text {alc-tail] }}\right)\left(\mathrm{CH}_{2}^{[\mathrm{OH}]}\right)(\mathrm{OH})$ & $330-339$ & VLE & 8 & 1.00 & Gmehling and Onken (1977) \\
\hline 1-propanol & $\mathrm{CH}_{\mathrm{n}}^{[\text {alc-tail }]}, \mathrm{CH}_{\mathrm{n}}^{[\mathrm{OH}]}, \mathrm{OH}$ & $\left(\mathrm{CH}_{3}^{[\text {alc-tail }]}\right)\left(\mathrm{CH}_{2}^{[\text {alc-tail }]}\right)\left(\mathrm{CH}_{2}^{[\mathrm{OH}]}\right)(\mathrm{OH})$ & $345-355$ & VLE & 8 & 1.00 & Gmehling and Onken (1977) \\
\hline 1-propanol & $\mathrm{CH}_{\mathrm{n}}^{[\text {alc-tail }]}, \mathrm{CH}_{\mathrm{n}}^{[\mathrm{OH}]}, \mathrm{OH}$ & $\left(\mathrm{CH}_{3}^{[\text {[alc-tail }]}\right)\left(\mathrm{CH}_{2}^{[\text {[alc-tail }]}\right)\left(\mathrm{CH}_{2}^{[\mathrm{OH}]}\right)(\mathrm{OH})$ & $355-365$ & VLE & 8 & 1.00 & Gmehling and Onken (1977) \\
\hline 1-propanol & $\mathrm{CH}_{\mathrm{n}}^{[\text {alc-tail }]}, \mathrm{CH}_{\mathrm{n}}^{[\mathrm{OH}]}, \mathrm{OH}$ & $\left(\mathrm{CH}_{3}^{[\text {[alc-tail }]}\right)\left(\mathrm{CH}_{2}^{[\text {[alc-tail }]}\right)\left(\mathrm{CH}_{2}^{[\mathrm{OH}]}\right)(\mathrm{OH})$ & $361-372$ & VLE & 8 & 1.00 & Gmehling and Onken (1977) \\
\hline 2-propanol & $\mathrm{CH}_{\mathrm{n}}^{\text {[alc] }}, \mathrm{CH}_{\mathrm{n}}^{[\mathrm{OH}]}, \mathrm{OH}$ & $\left(\mathrm{CH}_{3}^{[\mathrm{alc}]}\right)_{2}\left(\mathrm{CH}^{[\mathrm{OH}]}\right)(\mathrm{OH})$ & $271-273$ & SLE & 9 & 5.00 & Okamoto et al. (1978) \\
\hline 2-propanol & $\mathrm{CH}_{\mathrm{n}}^{\text {[alc] }}, \mathrm{CH}_{\mathrm{n}}^{[\mathrm{OH}]}, \mathrm{OH}$ & $\left(\mathrm{CH}_{3}^{[\text {alc }]}\right)_{2}\left(\mathrm{CH}^{[\mathrm{OH}]}\right)(\mathrm{OH})$ & $272-273$ & SLE & 17 & 5.00 & Webb and Lindsley (1934) \\
\hline 2-propanol & $\mathrm{CH}_{\mathrm{n}}^{[\text {alc }]}, \mathrm{CH}_{\mathrm{n}}^{[\mathrm{OH}]}, \mathrm{OH}$ & $\left(\mathrm{CH}_{3}^{\text {[alc }]}\right)_{2}\left(\mathrm{CH}^{[\mathrm{OH}]}\right)(\mathrm{OH})$ & $353-372$ & VLE & 24 & 1.00 & Gmehling and Onken (1977) \\
\hline 2-propanol & $\mathrm{CH}_{\mathrm{n}}^{\text {[alc] }}, \mathrm{CH}_{\mathrm{n}}^{[\mathrm{OH}]}, \mathrm{OH}$ & $\left(\mathrm{CH}_{3}^{[\text {alc }]}\right)_{2}\left(\mathrm{CH}^{[\mathrm{OH}]}\right)(\mathrm{OH})$ & $341-357$ & VLE & 19 & 1.00 & Gmehling and Onken (2003a) \\
\hline 2-propanol & $\mathrm{CH}_{\mathrm{n}}^{[\text {alc }]}, \mathrm{CH}_{\mathrm{n}}^{[\mathrm{OH}]}, \mathrm{OH}$ & $\left(\mathrm{CH}_{3}^{[\text {alc }]}\right)_{2}\left(\mathrm{CH}^{[\mathrm{OH}]}\right)(\mathrm{OH})$ & $325-340$ & VLE & 19 & 1.00 & Gmehling and Onken (2003a) \\
\hline 2-propanol & $\mathrm{CH}_{\mathrm{n}}^{\text {[alc] }}, \mathrm{CH}_{\mathrm{n}}^{[\mathrm{OH}]}, \mathrm{OH}$ & $\left(\mathrm{CH}_{3}^{[\text {alc }]}\right)_{2}\left(\mathrm{CH}^{[\mathrm{OH}]}\right)(\mathrm{OH})$ & 328 & VLE & 8 & 1.00 & Gmehling and Onken (1977) \\
\hline 2-propanol & $\mathrm{CH}_{\mathrm{n}}^{[\mathrm{alc}]}, \mathrm{CH}_{\mathrm{n}}^{[\mathrm{OH}]}, \mathrm{OH}$ & $\left(\mathrm{CH}_{3}^{\text {[alc] }}\right)_{2}\left(\mathrm{CH}^{[\mathrm{OH}]}\right)(\mathrm{OH})$ & 318 & VLE & 8 & 1.00 & Gmehling and Onken (1977) \\
\hline 2-propanol & $\mathrm{CH}_{\mathrm{n}}^{[\mathrm{alc}]}, \mathrm{CH}_{\mathrm{n}}^{[\mathrm{OH}]}, \mathrm{OH}$ & $\left(\mathrm{CH}_{3}^{[\mathrm{alc}]}\right)_{2}\left(\mathrm{CH}^{[\mathrm{OH}]}\right)(\mathrm{OH})$ & 308 & VLE & 8 & 1.00 & Gmehling and Onken (1977) \\
\hline 1-butanol & $\mathrm{CH}_{\mathrm{n}}^{[\text {[alc-tail }]}, \mathrm{CH}_{\mathrm{n}}^{[\mathrm{OH}]}, \mathrm{OH}$ & $\left(\mathrm{CH}_{3}^{[\text {[alc-tail }]}\right)\left(\mathrm{CH}_{2}^{[\text {alc-tail }]}\right)_{2}\left(\mathrm{CH}_{2}^{[\mathrm{OH}]}\right)(\mathrm{OH})$ & $271-273$ & SLE & 22 & 5.00 & Knight (1962) \\
\hline 1-butanol & $\mathrm{CH}_{\mathrm{n}}^{[\mathrm{alc}-\text { tail }]}, \mathrm{CH}_{\mathrm{n}}^{[\mathrm{OH}]}, \mathrm{OH}$ & $\left(\mathrm{CH}_{3}^{\text {[alc-tail }]}\right)\left(\mathrm{CH}_{2}^{\text {[alc-tail }]}\right)_{2}\left(\mathrm{CH}_{2}^{[\mathrm{OH}]}\right)(\mathrm{OH})$ & $200-273$ & SLE & 10 & 5.00 & Lohmann et al. (1997) \\
\hline 1-butanol & $\mathrm{CH}_{\mathrm{n}}^{[\text {alc-tail }]}, \mathrm{CH}_{\mathrm{n}}^{[\mathrm{OH}]}, \mathrm{OH}$ & $\left(\mathrm{CH}_{3}^{[\text {alc-tail }]}\right)\left(\mathrm{CH}_{2}^{[\text {[alc-tail }]}\right)_{2}\left(\mathrm{CH}_{2}^{[\mathrm{OH}]}\right)(\mathrm{OH})$ & $366-384$ & VLE & 12 & 1.00 & Gmehling and Onken (1977) \\
\hline 1-butanol & $\mathrm{CH}_{\mathrm{n}}^{[\text {alc-tail }]}, \mathrm{CH}_{\mathrm{n}}^{[\mathrm{OH}]}, \mathrm{OH}$ & $\left(\mathrm{CH}_{3}^{\text {[alc-tail }]}\right)\left(\mathrm{CH}_{2}^{\text {[alc-tail] }}\right)_{2}\left(\mathrm{CH}_{2}^{[\mathrm{OH}]}\right)(\mathrm{OH})$ & $365-382$ & VLE & 8 & 1.00 & Gmehling and Onken (2003a) \\
\hline 1-butanol & $\mathrm{CH}_{\mathrm{n}}^{\text {[alc-tail }]}, \mathrm{CH}_{\mathrm{n}}^{[\mathrm{OH}]}, \mathrm{OH}$ & $\left(\mathrm{CH}_{3}^{[\text {alc-tail }]}\right)\left(\mathrm{CH}_{2}^{\text {[alc-tail }]}\right)_{2}\left(\mathrm{CH}_{2}^{[\mathrm{OH}]}\right)(\mathrm{OH})$ & 323 & VLE & 4 & 1.00 & Gmehling et al. (1988) \\
\hline 1-butanol & $\mathrm{CH}_{\mathrm{n}}^{[\mathrm{alc}-\text { tail }]}, \mathrm{CH}_{\mathrm{n}}^{[\mathrm{OH}]}, \mathrm{OH}$ & $\left(\mathrm{CH}_{3}^{\text {[alc-tail }]}\right)\left(\mathrm{CH}_{2}^{\text {[alc-tail] }}\right)_{2}\left(\mathrm{CH}_{2}^{[\mathrm{OH}]}\right)(\mathrm{OH})$ & 343 & VLE & 4 & 1.00 & Gmehling et al. (1988) \\
\hline 1-butanol & $\mathrm{CH}_{\mathrm{n}}^{[\text {alc-tail }]}, \mathrm{CH}_{\mathrm{n}}^{[\mathrm{OH}]}, \mathrm{OH}$ & $\left(\mathrm{CH}_{3}^{\text {[alc-tail }]}\right)\left(\mathrm{CH}_{2}^{[\text {alc-tail }]}\right)_{2}\left(\mathrm{CH}_{2}^{[\mathrm{OOH}]}\right)(\mathrm{OH})$ & 363 & VLE & 4 & 1.00 & Gmehling et al. (1988) \\
\hline 2-butanol & $\mathrm{CH}_{\mathrm{n}}^{[\text {alc }]}, \mathrm{CH}_{\mathrm{n}}^{[\text {alc-tail }]}, \mathrm{CH}_{\mathrm{n}}^{[\mathrm{OH}]}, \mathrm{OH}$ & $\left(\mathrm{CH}_{3}^{\text {[alc] }]}\right)\left(\mathrm{CH}_{3}^{\text {[alc-tail }]}\right)\left(\mathrm{CH}_{2}^{[\text {alc-tail }]}\right)\left(\mathrm{CH}^{[\mathrm{OH}]}\right)(\mathrm{OH})$ & $215-259$ & SLE & 10 & 5.00 & Lohmann et al. (1997) \\
\hline 2-butanol & $\mathrm{CH}_{\mathrm{n}}^{[\text {alc }]}, \mathrm{CH}_{\mathrm{n}}^{[\mathrm{alc}-\text { tail }]}, \mathrm{CH}_{\mathrm{n}}^{[\mathrm{OH}]}, \mathrm{OH}$ & $\left(\mathrm{CH}_{3}^{[\text {alc }]}\right)\left(\mathrm{CH}_{3}^{[\text {alc-tail }]}\right)\left(\mathrm{CH}_{2}^{[\text {alc-tail] }}\right)\left(\mathrm{CH}^{[\mathrm{OH}]}\right)(\mathrm{OH})$ & $330-335$ & VLE & 11 & 1.00 & Gmehling and Onken (2003a) \\
\hline 2-butanol & $\mathrm{CH}_{\mathrm{n}}^{[\text {alc }]}, \mathrm{CH}_{\mathrm{n}}^{[\text {alc-tail }]}, \mathrm{CH}_{\mathrm{n}}^{[\mathrm{OH}]}, \mathrm{OH}$ & $\left(\mathrm{CH}_{3}^{\text {[alc] }]}\right)\left(\mathrm{CH}_{3}^{\text {[alc-tail }]}\right)\left(\mathrm{CH}_{2}^{\text {[alc-tail }]}\right)\left(\mathrm{CH}^{[\mathrm{OH}]}\right)(\mathrm{OH})$ & $345-351$ & VLE & 11 & 1.00 & Gmehling and Onken (2003a) \\
\hline 2-butanol & $\mathrm{CH}_{\mathrm{n}}^{[\text {alc }]}, \mathrm{CH}_{\mathrm{n}}^{[\mathrm{alc}-\text { tail }]}, \mathrm{CH}_{\mathrm{n}}^{[\mathrm{OH}]}, \mathrm{OH}$ & $\left(\mathrm{CH}_{3}^{[\text {alc }]}\right)\left(\mathrm{CH}_{3}^{\text {[alc-tail }]}\right)\left(\mathrm{CH}_{2}^{[\text {alc-tail] }}\right)\left(\mathrm{CH}^{[\mathrm{OH}]}\right)(\mathrm{OH})$ & $355-360$ & VLE & 11 & 1.00 & Gmehling and Onken (2003a) \\
\hline 2-butanol & $\mathrm{CH}_{\mathrm{n}}^{[\text {alc }]}, \mathrm{CH}_{\mathrm{n}}^{[\text {alc-tail }]}, \mathrm{CH}_{\mathrm{n}}^{[\mathrm{OH}]}, \mathrm{OH}$ & $\left(\mathrm{CH}_{3}^{\text {[alc] }}\right)\left(\mathrm{CH}_{3}^{\text {[alc-tail }]}\right)\left(\mathrm{CH}_{2}^{\text {[alc-tail }]}\right)\left(\mathrm{CH}^{[\mathrm{OH}]}\right)(\mathrm{OH})$ & $360-367$ & VLE & 20 & 1.00 & Gmehling and Onken (2003a) \\
\hline 2-butanol & $\mathrm{CH}_{\mathrm{n}}^{[\text {alc }]}, \mathrm{CH}_{\mathrm{n}}^{[\mathrm{alc}-\text { tail }]}, \mathrm{CH}_{\mathrm{n}}^{[\mathrm{OH}]}, \mathrm{OH}$ & $\left(\mathrm{CH}_{3}^{[\text {alc }]}\right)\left(\mathrm{CH}_{3}^{\text {[alc-tail] }}\right)\left(\mathrm{CH}_{2}^{[\text {alc-tail] }}\right)\left(\mathrm{CH}^{[\mathrm{OH}]}\right)(\mathrm{OH})$ & $361-367$ & VLE & 11 & 1.00 & Gmehling and Onken (2003a) \\
\hline isobutanol & $\mathrm{CH}_{\mathrm{n}}^{[\text {alc-tail }]}, \mathrm{CH}_{\mathrm{n}}^{[\mathrm{OH}]}, \mathrm{OH}$ & $\left(\mathrm{CH}_{3}^{\text {[alc-tail }]}\right)_{2}\left(\mathrm{CH}^{[\text {alc-tail }]}\right)\left(\mathrm{CH}_{2}^{[\mathrm{OH}]}\right)(\mathrm{OH})$ & $225-273$ & SLE & 10 & 5.00 & Lohmann et al. (1997) \\
\hline tert-butanol & $\mathrm{CH}_{\mathrm{n}}^{[\mathrm{alc}]}, \mathrm{CH}_{\mathrm{n}}^{[\mathrm{OH}]}, \mathrm{OH}$ & $\left(\mathrm{CH}_{3}^{\text {[alc] }}\right)_{3}\left(\mathrm{C}^{[\mathrm{OH}]}\right)(\mathrm{OH})$ & $266-273$ & SLE & 34 & 5.00 & Knight (1962) \\
\hline tert-butanol & $\mathrm{CH}_{\mathrm{n}}^{[\mathrm{alc}]}, \mathrm{CH}_{\mathrm{n}}^{[\mathrm{OH}]}, \mathrm{OH}$ & $\left(\mathrm{CH}_{3}^{\text {[alc }]}\right)_{3}\left(\mathrm{C}^{[\mathrm{OH}]}\right)(\mathrm{OH})$ & $353-364$ & VLE & 15 & 1.00 & Gmehling et al. (1981) \\
\hline tert-butanol & $\mathrm{CH}_{\mathrm{n}}^{[\mathrm{alc}]}, \mathrm{CH}_{\mathrm{n}}^{[\mathrm{OH}]}, \mathrm{OH}$ & $\left(\mathrm{CH}_{3}^{[\mathrm{alc}]}\right)_{3}\left(\mathrm{C}^{[\mathrm{OH}]}\right)(\mathrm{OH})$ & $331-339$ & VLE & 15 & 1.00 & Gmehling and Onken (1977) \\
\hline tert-butanol & $\mathrm{CH}_{\mathrm{n}}^{\text {[alc] }}, \mathrm{CH}_{\mathrm{n}}^{[\mathrm{OH}]}, \mathrm{OH}$ & $\left(\mathrm{CH}_{3}^{[\mathrm{alc}]}\right)_{3}\left(\mathrm{C}^{[\mathrm{OH}]}\right)(\mathrm{OH})$ & $308-317$ & VLE & 17 & 1.00 & Gmehling and Onken (1977) \\
\hline tert-butanol & $\mathrm{CH}_{\mathrm{n}}^{[\mathrm{alc}]}, \mathrm{CH}_{\mathrm{n}}^{[\mathrm{OH}]}, \mathrm{OH}$ & $\left(\mathrm{CH}_{3}^{[\mathrm{alc}]}\right)_{3}\left(\mathrm{C}^{[\mathrm{OH}]}\right)(\mathrm{OH})$ & 353 & VLE & 9 & 1.00 & Gmehling and Onken (2003a) \\
\hline tert-butanol & $\mathrm{CH}_{\mathrm{n}}^{[\text {alc }]}, \mathrm{CH}_{\mathrm{n}}^{[\mathrm{OH}]}, \mathrm{OH}$ & $\left(\mathrm{CH}_{3}^{\text {[alc }]}\right)_{3}\left(\mathrm{C}^{[\mathrm{OH}]}\right)(\mathrm{OH})$ & 343 & VLE & 9 & 1.00 & Gmehling and Onken (2003a) \\
\hline tert-butanol & $\mathrm{CH}_{\mathrm{n}}^{\text {[alc }]}, \mathrm{CH}_{\mathrm{n}}^{[\mathrm{OH}]}, \mathrm{OH}$ & $\left(\mathrm{CH}_{3}^{[\text {alc }]}\right)_{3}\left(\mathrm{C}^{[\mathrm{OH}]}\right)(\mathrm{OH})$ & 303 & VLE & 16 & 1.00 & Gmehling and Onken (2003a) \\
\hline glycerol & $\mathrm{CH}_{\mathrm{n}}^{[\mathrm{OH}]}, \mathrm{OH}$ & $\left(\mathrm{CH}_{2}^{[\mathrm{OH}]}\right)_{2}\left(\mathrm{CH}^{[\mathrm{OH}]}\right)(\mathrm{OH})_{3}$ & $189-231$ & $a_{\mathrm{W}}\left(T_{\mathrm{hom}}\right)^{\mathrm{a}}$ & 10 & 1.00 & Kanno et al. (2004) \\
\hline glycerol & $\mathrm{CH}_{\mathrm{n}}^{[\mathrm{OH}]}, \mathrm{OH}$ & $\left(\mathrm{CH}_{2}^{[\mathrm{OH}]}\right)_{2}\left(\mathrm{CH}^{[\mathrm{OH}]}\right)(\mathrm{OH})_{3}$ & $225-271$ & SLE & 7 & 5.00 & Ross (1954) \\
\hline glycerol & $\mathrm{CH}_{\mathrm{n}}^{[\mathrm{OH}]}, \mathrm{OH}$ & $\left(\mathrm{CH}_{2}^{[\mathrm{OH}]}\right)_{2}\left(\mathrm{CH}^{[\mathrm{OH}]}\right)(\mathrm{OH})_{3}$ & $232-271$ & SLE & 10 & 5.00 & Olsen et al. (1930) \\
\hline glycerol & $\mathrm{CH}_{\mathrm{n}}^{[\mathrm{OH}]}, \mathrm{OH}$ & $\left(\mathrm{CH}_{2}^{[\mathrm{OH}]}\right)_{2}\left(\mathrm{CH}^{[\mathrm{OH}]}\right)(\mathrm{OH})_{3}$ & $258-272$ & SLE & 5 & 5.00 & Lerici et al. (2006) \\
\hline glycerol & $\mathrm{CH}_{\mathrm{n}}^{[\mathrm{OH}]}, \mathrm{OH}$ & $\left(\mathrm{CH}_{2}^{[\mathrm{OH}]}\right)_{2}\left(\mathrm{CH}^{[\mathrm{OH}]}\right)(\mathrm{OH})_{3}$ & $255-291$ & SLE(org) ${ }^{\mathrm{d}}$ & 11 & 0.20 & Pushin and Glagoleva (1922) \\
\hline glycerol & $\mathrm{CH}_{\mathrm{n}}^{[\mathrm{OH}]}, \mathrm{OH}$ & $\left(\mathrm{CH}_{2}^{[\mathrm{OH}]}\right)_{2}\left(\mathrm{CH}^{[\mathrm{OH}]}\right)(\mathrm{OH})_{3}$ & 289 & $a_{\mathrm{w}}($ bulk) & 15 & 0.0 & this work \\
\hline glycerol & $\mathrm{CH}_{\mathrm{n}}^{[\mathrm{OH}]}, \mathrm{OH}$ & $\left(\mathrm{CH}_{2}^{[\mathrm{OH}]}\right)_{2}\left(\mathrm{CH}^{[\mathrm{OH}]}\right)(\mathrm{OH})_{3}$ & 298 & $a_{\mathrm{W}}$ (bulk) & 15 & 0.0 & this work \\
\hline glycerol & $\mathrm{CH}_{\mathrm{n}}^{[\mathrm{OH}]}, \mathrm{OH}$ & $\left(\mathrm{CH}_{2}^{[\mathrm{OH}]}\right)_{2}\left(\mathrm{CH}^{[\mathrm{OH}]}\right)(\mathrm{OH})_{3}$ & 313 & $a_{\mathrm{W}}$ (bulk) & 15 & 1.0 & this work \\
\hline glycerol & $\mathrm{CH}_{\mathrm{n}}^{[\mathrm{OH}]}, \mathrm{OH}$ & $\left(\mathrm{CH}_{2}^{[\mathrm{OH}]}\right)_{2}\left(\mathrm{CH}^{[\mathrm{OH}]}\right)(\mathrm{OH})_{3}$ & $347-421$ & VLE & 8 & 0.001 & Soujanya et al. (2010) \\
\hline glycerol & $\mathrm{CH}_{\mathrm{n}}^{[\mathrm{OH}]}, \mathrm{OH}$ & $\left(\mathrm{CH}_{2}^{[\mathrm{OH}]}\right)_{2}\left(\mathrm{CH}^{[\mathrm{OH}]}\right)(\mathrm{OH})_{3}$ & $353-447$ & VLE & 9 & 0.001 & Soujanya et al. (2010) \\
\hline glycerol & $\mathrm{CH}_{\mathrm{n}}^{[\mathrm{OH}]}, \mathrm{OH}$ & $\left(\mathrm{CH}_{2}^{[\mathrm{OH}]}\right)_{2}\left(\mathrm{CH}^{[\mathrm{OH}]}\right)(\mathrm{OH})_{3}$ & $358-454$ & VLE & 10 & 0.001 & Soujanya et al. (2010) \\
\hline glycerol & $\mathrm{CH}_{\mathrm{n}}^{[\mathrm{OH}]}, \mathrm{OH}$ & $\left(\mathrm{CH}_{2}^{[\mathrm{OH}]}\right)_{2}\left(\mathrm{CH}^{[\mathrm{OH}]}\right)(\mathrm{OH})_{3}$ & $364-484$ & VLE & 9 & 0.001 & Soujanya et al. (2010) \\
\hline glycerol & $\mathrm{CH}_{\mathrm{n}}^{[\mathrm{OH}]}, \mathrm{OH}$ & $\left(\mathrm{CH}_{2}^{[\mathrm{OH}]}\right)_{2}\left(\mathrm{CH}^{[\mathrm{OH}]}\right)(\mathrm{OH})_{3}$ & $373-410$ & VLE & 7 & 0.001 & Soujanya et al. (2010) \\
\hline 1,2-ethanediol & $\mathrm{CH}_{\mathrm{n}}^{[\mathrm{OH}]}, \mathrm{OH}$ & $\left(\mathrm{CH}_{2}^{[\mathrm{OH}]}\right)_{2}(\mathrm{OH})_{2}$ & $220-270$ & SLE & 6 & 5.00 & Dykyj et al. (1956) \\
\hline 1,2-ethanediol & $\mathrm{CH}_{\mathrm{n}}^{[\mathrm{OH}]}, \mathrm{OH}$ & $\left(\mathrm{CH}_{2}^{[\mathrm{OH}]}\right)_{2}(\mathrm{OH})_{2}$ & $271-273$ & SLE & 10 & 5.00 & Okamoto et al. (1978) \\
\hline 1,2-ethanediol & $\mathrm{CH}_{\mathrm{n}}^{[\mathrm{OH}]}, \mathrm{OH}$ & $\left(\mathrm{CH}_{2}^{[\mathrm{OH}]}\right)_{2}(\mathrm{OH})_{2}$ & $225-267$ & SLE & 7 & 5.00 & Ott et al. (1972) \\
\hline 1,2-ethanediol & $\mathrm{CH}_{\mathrm{n}}^{[\mathrm{OH}]}, \mathrm{OH}$ & $\left(\mathrm{CH}_{2}^{[\mathrm{OH}]}\right)_{2}(\mathrm{OH})_{2}$ & $224-273$ & SLE & 7 & 2.00 & Ott et al. (1972) \\
\hline 1,2-ethanediol & $\mathrm{CH}_{\mathrm{n}}^{[\mathrm{OH}]}, \mathrm{OH}$ & $\left(\mathrm{CH}_{2}^{[\mathrm{OH}]}\right)_{2}(\mathrm{OH})_{2}$ & $223-270$ & SLE & 6 & 5.00 & Clendenning (1946) \\
\hline
\end{tabular}


Table 1. Continued.

\begin{tabular}{|c|c|c|c|c|c|c|c|}
\hline Organic compounds & Org. main groups & Chemical formula (subgroups) & $T(\mathrm{~K})$ & Data type & $N_{d}$ & $w_{d}^{\text {init }}$ & Reference \\
\hline 1,2-ethanediol & $\mathrm{CH}_{\mathrm{n}}^{[\mathrm{OH}]}, \mathrm{OH}$ & $\left(\mathrm{CH}_{2}^{[\mathrm{OH}]}\right)_{2}(\mathrm{OH})_{2}$ & $230-260$ & SLE(org) ${ }^{\mathrm{d}}$ & 11 & 0.20 & Ott et al. (1972) \\
\hline 1,2-ethanediol & $\mathrm{CH}_{\mathrm{n}}^{[\mathrm{OH}]}, \mathrm{OH}$ & $\left(\mathrm{CH}_{2}^{[\mathrm{OOH}]}\right)_{2}(\mathrm{OH})_{2}$ & 298.15 & $a_{\mathrm{W}}($ bulk) & 14 & 0.0 & Marcolli and Peter (2005) \\
\hline 1,2-ethanediol & $\mathrm{CH}_{\mathrm{n}}^{[\mathrm{OH}]}, \mathrm{OH}$ & $\left(\mathrm{CH}_{2}^{[\mathrm{OH}]}\right)_{2}(\mathrm{OH})_{2}$ & 323 & VLE & 19 & 1.00 & Gmehling et al. (1988) \\
\hline 1,2-ethanediol & $\mathrm{CH}_{\mathrm{n}}^{[\mathrm{OH}]}, \mathrm{OH}$ & $\left(\mathrm{CH}_{2}^{[\mathrm{OH}]}\right)_{2}(\mathrm{OH})_{2}$ & 333 & VLE & 20 & 1.00 & Gmehling et al. (1988) \\
\hline 1,2-ethanediol & $\mathrm{CH}_{\mathrm{n}}^{[\mathrm{OH}]}, \mathrm{OH}$ & $\left(\mathrm{CH}_{2}^{[\mathrm{OH}]}\right)_{2}(\mathrm{OH})_{2}$ & 338 & VLE & 10 & 1.00 & Gmehling et al. (1988) \\
\hline 1,2-ethanediol & $\mathrm{CH}_{\mathrm{n}}^{[\mathrm{OH}]}, \mathrm{OH}$ & $\left(\mathrm{CH}_{2}^{[\mathrm{OH}]}\right)_{2}(\mathrm{OH})_{2}$ & 343 & VLE & 15 & 0.20 & Gmehling and Onken (2003a) \\
\hline 1,2-ethanediol & $\mathrm{CH}_{\mathrm{n}}^{[\mathrm{OH}]}, \mathrm{OH}$ & $\left(\mathrm{CH}_{2}^{[\mathrm{OH}]}\right)_{2}(\mathrm{OH})_{2}$ & 363 & VLE & 12 & 1.00 & Gmehling et al. (1988) \\
\hline 1,2-ethanediol & $\mathrm{CH}_{\mathrm{n}}^{[\mathrm{OH}]}, \mathrm{OH}$ & $\left(\mathrm{CH}_{2}^{[\mathrm{OH}]}\right)_{2}(\mathrm{OH})_{2}$ & $359-437$ & VLE & 9 & 1.00 & Gmehling and Onken (2003a) \\
\hline 1,2-propanediol & $\mathrm{CH}_{\mathrm{n}}^{[\mathrm{alc}]}, \mathrm{CH}_{\mathrm{n}}^{[\mathrm{OH}]}, \mathrm{OH}$ & $\left(\mathrm{CH}_{3}^{[\mathrm{alc}]}\right)\left(\mathrm{CH}_{2}^{[\mathrm{OH}]}\right)\left(\mathrm{CH}^{[\mathrm{OH}]}\right)(\mathrm{OH})_{2}$ & $207-270$ & SLE & 7 & 5.00 & Ross (1954) \\
\hline 1,2 propanediol & $\mathrm{CH}_{\mathrm{n}}^{[\mathrm{alc}]}, \mathrm{CH}_{\mathrm{n}}^{[\mathrm{OH}]}, \mathrm{OH}$ & $\left(\mathrm{CH}_{3}^{[\mathrm{lalc}]}\right)\left(\mathrm{CH}_{2}^{[\mathrm{OH}]}\right)\left(\mathrm{CH}^{[\mathrm{OH}]}\right)(\mathrm{OH})_{2}$ & $216-271$ & SLE & 12 & 5.00 & Boese et al. (1953) \\
\hline 1,2-propanediol & $\mathrm{CH}_{\mathrm{n}}^{[\text {alc }]}, \mathrm{CH}_{\mathrm{n}}^{[\mathrm{OH}]}, \mathrm{OH}$ & $\left(\mathrm{CH}_{3}^{[\text {[alc }]}\right)\left(\mathrm{CH}_{2}^{[\mathrm{OH}]}\right)\left(\mathrm{CH}^{[\mathrm{OH}]}\right)(\mathrm{OH})_{2}$ & 298 & $a_{\mathrm{W}}$ (bulk) & 13 & 0.0 & Marcolli and Peter (2005) \\
\hline 1,2-propanediol & $\mathrm{CH}_{\mathrm{n}}^{[\text {[alc }]}, \mathrm{CH}_{\mathrm{n}}^{[\mathrm{OH}]}, \mathrm{OH}$ & $\left(\mathrm{CH}_{3}^{[\mathrm{alc}]}\right)\left(\mathrm{CH}_{2}^{[\mathrm{OH}]}\right)\left(\mathrm{CH}^{[\mathrm{OH}]}\right)(\mathrm{OH})_{2}$ & 288 & VLE & 5 & 1.00 & Gmehling et al. (1988) \\
\hline 1,2-propanediol & $\mathrm{CH}_{\mathrm{n}}^{[\text {[alc }]}, \mathrm{CH}_{\mathrm{n}}^{[\mathrm{OH}]}, \mathrm{OH}$ & $\left(\mathrm{CH}_{3}^{[\mathrm{alc}]}\right)\left(\mathrm{CH}_{2}^{[\mathrm{OH}]}\right)\left(\mathrm{CH}^{[\mathrm{OH}]}\right)(\mathrm{OH})_{2}$ & 298 & VLE & 5 & 0.0 & Gmehling et al. (1988) \\
\hline 1,2-propanediol & $\mathrm{CH}_{\mathrm{n}}^{[\mathrm{alc}]}, \mathrm{CH}_{\mathrm{n}}^{[\mathrm{OH}]}, \mathrm{OH}$ & $\left(\mathrm{CH}_{3}^{\text {[alc] }}\right)\left(\mathrm{CH}_{2}^{2 \mathrm{OH}]}\right)\left(\mathrm{CH}^{[\mathrm{OH}]}\right)(\mathrm{OH})_{2}$ & 303 & VLE & 5 & 1.00 & Gmehling et al. (1988) \\
\hline 1,2-propanediol & $\mathrm{CH}_{\mathrm{n}}^{[\mathrm{alc}]}, \mathrm{CH}_{\mathrm{n}}^{[\mathrm{OH}]}, \mathrm{OH}$ & $\left(\mathrm{CH}_{3}^{\text {[alc] }}\right)\left(\mathrm{CH}_{2}^{[\mathrm{OH}]}\right)\left(\mathrm{CH}^{[\mathrm{OH}]}\right)(\mathrm{OH})_{2}$ & 308 & VLE & 5 & 1.00 & Gmehling et al. (1988) \\
\hline 1,2 -propanediol & $\mathrm{CH}_{\mathrm{n}}^{\text {[alc] }}, \mathrm{CH}_{\mathrm{n}}^{\mathrm{OOH}]}, \mathrm{OH}$ & $\left(\mathrm{CH}_{3}^{[\mathrm{alc}]}\right)\left(\mathrm{CH}_{2}^{[\mathrm{OH}]}\right)\left(\mathrm{CH}^{[\mathrm{OH}]}\right)(\mathrm{OH})_{2}$ & 318 & VLE & 5 & 1.00 & Gmehling et al. (1988) \\
\hline 1,2-propanediol & $\mathrm{CH}_{\mathrm{n}}^{[\text {alc }]}, \mathrm{CH}_{\mathrm{n}}^{[\mathrm{OH}]}, \mathrm{OH}$ & $\left(\mathrm{CH}_{3}^{[\mathrm{alc}]}\right)\left(\mathrm{CH}_{2}^{[\mathrm{OH}]}\right)\left(\mathrm{CH}^{[\mathrm{OH}]}\right)(\mathrm{OH})_{2}$ & 323 & VLE & 5 & 1.00 & Gmehling et al. (1988) \\
\hline 1,2-propanediol & $\mathrm{CH}_{\mathrm{n}}^{[\text {alc }]}, \mathrm{CH}_{\mathrm{n}}^{[\mathrm{OH}]}, \mathrm{OH}$ & $\left(\mathrm{CH}_{3}^{[\mathrm{alc}]}\right)\left(\mathrm{CH}_{2}^{[\mathrm{OH}]}\right)\left(\mathrm{CH}^{[\mathrm{OH}]}\right)(\mathrm{OH})_{2}$ & 353 & VLE & 8 & 1.00 & Gmehling and Onken (2003a) \\
\hline 1,2-propanediol & $\mathrm{CH}_{\mathrm{n}}^{[\text {[alc] }]}, \mathrm{CH}_{\mathrm{n}}^{[\mathrm{OOH}]}, \mathrm{OH}$ & $\left(\mathrm{CH}_{3}^{\mathrm{alc}]}\right)\left(\mathrm{CH}_{2}^{[\mathrm{OH}]}\right)\left(\mathrm{CH}^{[\mathrm{OH}]}\right)(\mathrm{OH})_{2}$ & 373 & VLE & 6 & 0.10 & Gmehling and Onken (2003a) \\
\hline 1,2-propanediol & $\mathrm{CH}_{\mathrm{n}}^{[\text {[alc }]}, \mathrm{CH}_{\mathrm{n}}^{[\mathrm{OH}]}, \mathrm{OH}$ & $\left(\mathrm{CH}_{3}^{[\mathrm{lalc}]}\right)\left(\mathrm{CH}_{2}^{[\mathrm{OH}]}\right)\left(\mathrm{CH}^{[\mathrm{OH}]}\right)(\mathrm{OH})_{2}$ & 383 & VLE & 11 & 1.00 & Gmehling and Onken (2003a) \\
\hline 1,2-propanediol & $\mathrm{CH}_{\mathrm{n}}^{[\mathrm{alc}]}, \mathrm{CH}_{\mathrm{n}}^{[\mathrm{OH}]}, \mathrm{OH}$ & $\left(\mathrm{CH}_{3}^{[\text {alc }]}\right)\left(\mathrm{CH}_{2}^{[\mathrm{OH}]}\right)\left(\mathrm{CH}^{[\mathrm{OH}]}\right)(\mathrm{OH})_{2}$ & 395 & VLE & 9 & 1.00 & Gmehling and Onken (2003a) \\
\hline 1,3-propanediol & $\mathrm{CH}_{\mathrm{n}}^{[\text {alc }]}, \mathrm{CH}_{\mathrm{n}}^{[\mathrm{OH}]}, \mathrm{OH}$ & $\left(\mathrm{CH}_{2}^{[\mathrm{alc}]}\right)\left(\mathrm{CH}_{2}^{[\mathrm{OH}]}\right)_{2}(\mathrm{OH})_{2}$ & $200-231$ & $a_{\mathrm{W}}\left(T_{\mathrm{hom}}\right)^{\mathrm{a}}$ & 4 & 1.00 & Ganbavale et al. (2014) \\
\hline 1,3-propanediol & $\mathrm{CH}_{\mathrm{n}}^{[\text {[alc }]}, \mathrm{CH}_{\mathrm{n}}^{[\mathrm{OH}]}, \mathrm{OH}$ & $\left(\mathrm{CH}_{2}^{[\mathrm{alc}]}\right)\left(\mathrm{CH}_{2}^{[\mathrm{OH}]}\right)_{2}(\mathrm{OH})_{2}$ & $256-270$ & SLE & 5 & 5.00 & Ganbavale et al. (2014) \\
\hline 1,3-propanediol & $\mathrm{CH}_{\mathrm{n}}^{[\text {[alc }]}, \mathrm{CH}_{\mathrm{n}}^{[\mathrm{OH}]}, \mathrm{OH}$ & $\left(\mathrm{CH}_{2}^{[\mathrm{falc}]}\right)\left(\mathrm{CH}_{2}^{[\mathrm{OH}]}\right)_{2}(\mathrm{OH})_{2}$ & $249-270$ & SLE & 5 & 5.00 & Ross (1954) \\
\hline 1,3-propanediol & $\mathrm{CH}_{\mathrm{n}}^{[\mathrm{alc}]}, \mathrm{CH}_{\mathrm{n}}^{[\mathrm{OH}]}, \mathrm{OH}$ & $\left(\mathrm{CH}_{2}^{[\mathrm{alc}]}\right)\left(\mathrm{CH}_{2}^{[\mathrm{OH}]}\right)_{2}(\mathrm{OH})_{2}$ & 298 & $a_{\mathrm{W}}($ bulk) & 13 & 0.00 & Marcolli and Peter (2005) \\
\hline 1,3-propanediol & $\mathrm{CH}_{\mathrm{n}}^{[\text {alc }]}, \mathrm{CH}_{\mathrm{n}}^{[\mathrm{OH}]}, \mathrm{OH}$ & $\left(\mathrm{CH}_{2}^{[\mathrm{alc}]}\right)\left(\mathrm{CH}_{2}^{[\mathrm{OOH}]}\right)_{2}(\mathrm{OH})_{2}$ & $343-442$ & VLE & 19 & 0.1 & Sanz et al. (2001) \\
\hline 1,3-propanediol & $\mathrm{CH}_{\mathrm{n}}^{[\text {[alc }]}, \mathrm{CH}_{\mathrm{n}}^{[\mathrm{OH}]}, \mathrm{OH}$ & $\left(\mathrm{CH}_{2}^{[\mathrm{falc}]}\right)\left(\mathrm{CH}_{2}^{[\mathrm{OH}]}\right)_{2}(\mathrm{OH})_{2}$ & $341-428$ & VLE & 12 & 1.00 & Mun and Lee (1999) \\
\hline 1,3-propanediol & $\mathrm{CH}_{\mathrm{n}}^{[\text {[alc }]}, \mathrm{CH}_{\mathrm{n}}^{[\mathrm{OH}]}, \mathrm{OH}$ & $\left(\mathrm{CH}_{2}^{[\mathrm{falc}]}\right)\left(\mathrm{CH}_{2}^{[\mathrm{OOH}]}\right)_{2}(\mathrm{OH})_{2}$ & $353-441$ & VLE & 18 & 0.20 & Mun and Lee (1999) \\
\hline 1,4-butanediol & $\mathrm{CH}_{\mathrm{n}}^{[\mathrm{alc}]}, \mathrm{CH}_{\mathrm{n}}^{[\mathrm{OH}]}, \mathrm{OH}$ & $\left(\mathrm{CH}_{2}^{\text {[alc] }}\right)_{2}\left(\mathrm{CH}_{2}^{[\mathrm{OH}]}\right)_{2}(\mathrm{OH})_{2}$ & $196-230$ & $a_{\mathrm{W}}\left(T_{\mathrm{hom}}\right)^{\mathrm{a}}$ & 4 & 1.00 & Zobrist et al. (2008) \\
\hline 1,4-butanediol & $\mathrm{CH}_{\mathrm{n}}^{[\mathrm{alc}]}, \mathrm{CH}_{\mathrm{n}}^{[\mathrm{OH}]}, \mathrm{OH}$ & $\left(\mathrm{CH}_{2}^{\text {[alc] }}\right)_{2}\left(\mathrm{CH}_{2}^{[\mathrm{OH}]}\right)_{2}(\mathrm{OH})_{2}$ & $258-270$ & SLE & 4 & 5.00 & Zobrist et al. (2008) \\
\hline 1,4-butanediol & $\mathrm{CH}_{\mathrm{n}}^{[\text {alc }]}, \mathrm{CH}_{\mathrm{n}}^{[\mathrm{OH}]}, \mathrm{OH}$ & $\left(\mathrm{CH}_{2}^{[\mathrm{Talc}]}\right)_{2}\left(\mathrm{CH}_{2}^{[\mathrm{fOH}]}\right)_{2}(\mathrm{OH})_{2}$ & 270 & $a_{\mathrm{W}}\left(p^{\mathrm{tot}}\right)^{\mathrm{e}}$ & 8 & 1.00 & Ganbavale et al. (2014) \\
\hline 1,4-butanediol & $\mathrm{CH}_{\mathrm{n}}^{[\text {[alc] }}, \mathrm{CH}_{\mathrm{n}}^{[\mathrm{OH}]}, \mathrm{OH}$ & $\left(\mathrm{CH}_{2}^{\text {[alc] }}\right)_{2}\left(\mathrm{CH}_{2}^{[\mathrm{OH}]}\right)_{2}(\mathrm{OH})_{2}$ & 273 & $a_{\mathrm{w}}\left(p^{\mathrm{tot}}\right)$ & 6 & 1.00 & Ganbavale et al. (2014) \\
\hline 1,4-butanediol & $\mathrm{CH}_{\mathrm{n}}^{[\text {alc }]}, \mathrm{CH}_{\mathrm{n}}^{[\mathrm{OH}]}, \mathrm{OH}$ & $\left(\mathrm{CH}_{2}^{[\mathrm{alcc}]}\right)_{2}\left(\mathrm{CH}_{2}^{[\mathrm{OH}]}\right)_{2}(\mathrm{OH})_{2}$ & 275 & $a_{\mathrm{w}}\left(p^{\mathrm{tot}}\right)$ & 8 & 1.00 & Ganbavale et al. (2014) \\
\hline 1,4-butanediol & $\mathrm{CH}_{\mathrm{n}}^{[\text {alc }]}, \mathrm{CH}_{\mathrm{n}}^{[\mathrm{OH}]}, \mathrm{OH}$ & $\left(\mathrm{CH}_{2}^{[\mathrm{alc}]}\right)_{2}\left(\mathrm{CH}_{2}^{[\mathrm{OOH}]}\right)_{2}(\mathrm{OH})_{2}$ & 278 & $a_{\mathrm{w}}\left(p^{\mathrm{tot}}\right)$ & 11 & 1.00 & Ganbavale et al. (2014) \\
\hline 1,4-butanediol & $\mathrm{CH}_{\mathrm{n}}^{[\text {alc }]}, \mathrm{CH}_{\mathrm{n}}^{[\mathrm{OH}]}, \mathrm{OH}$ & $\left(\mathrm{CH}_{2}^{[\mathrm{alc}]}\right)_{2}\left(\mathrm{CH}_{2}^{[\mathrm{OH}]}\right)_{2}(\mathrm{OH})_{2}$ & 280 & $a_{\mathrm{w}}\left(p^{\mathrm{tot}}\right)$ & 11 & 1.00 & Ganbavale et al. (2014) \\
\hline 1,4-butanediol & $\mathrm{CH}_{\mathrm{n}}^{[\mathrm{alc}]}, \mathrm{CH}_{\mathrm{n}}^{[\mathrm{OH}]}, \mathrm{OH}$ & $\left(\mathrm{CH}_{2}^{[\mathrm{alcc}]}\right)_{2}\left(\mathrm{CH}_{2}^{[\mathrm{OH}]}\right)_{2}(\mathrm{OH})_{2}$ & 283 & $a_{\mathrm{w}}\left(p^{\mathrm{tot}}\right)$ & 11 & 1.00 & Ganbavale et al. (2014) \\
\hline 1,4-butanediol & $\mathrm{CH}_{\mathrm{n}}^{[\mathrm{alc}]}, \mathrm{CH}_{\mathrm{n}}^{[\mathrm{OH}]}, \mathrm{OH}$ & $\left(\mathrm{CH}_{2}^{[\mathrm{alcc}]}\right)_{2}\left(\mathrm{CH}_{2}^{[\mathrm{fOH}]}\right)_{2}(\mathrm{OH})_{2}$ & 285 & $a_{\mathrm{w}}\left(p^{\mathrm{tot}}\right)$ & 11 & 1.00 & Ganbavale et al. (2014) \\
\hline 1,4-butanediol & $\mathrm{CH}_{\mathrm{n}}^{[\mathrm{alc}]}, \mathrm{CH}_{\mathrm{n}}^{[\mathrm{OH}]}, \mathrm{OH}$ & $\left(\mathrm{CH}_{2}^{[\mathrm{alc}]}\right)_{2}\left(\mathrm{CH}_{2}^{[\mathrm{OOH}]}\right)_{2}(\mathrm{OH})_{2}$ & 288 & $a_{\mathrm{w}}\left(p^{\mathrm{tot}}\right)$ & 11 & 0.00 & Ganbavale et al. (2014) \\
\hline 1,4-butanediol & $\mathrm{CH}_{\mathrm{n}}^{[\text {alc }]}, \mathrm{CH}_{\mathrm{n}}^{[\mathrm{OH}]}, \mathrm{OH}$ & $\left(\mathrm{CH}_{2}^{[\mathrm{alc}]}\right)_{2}\left(\mathrm{CH}_{2}^{[\mathrm{OH}]}\right)_{2}(\mathrm{OH})_{2}$ & 290 & $a_{\mathrm{w}}\left(p^{\mathrm{tot}}\right)$ & 11 & 0.00 & Ganbavale et al. (2014) \\
\hline 1,4-butanediol & $\mathrm{CH}_{\mathrm{n}}^{[\text {[alc }]}, \mathrm{CH}_{\mathrm{n}}^{[\mathrm{OH}]}, \mathrm{OH}$ & $\left(\mathrm{CH}_{2}^{[\mathrm{alc}]}\right)_{2}\left(\mathrm{CH}_{2}^{[\mathrm{fOH}]}\right)_{2}(\mathrm{OH})_{2}$ & 298 & $a_{\mathrm{W}}$ (bulk) & 16 & 0.00 & Marcolli and Peter (2005) \\
\hline 1,4-butanediol & $\mathrm{CH}_{\mathrm{n}}^{[\text {[alc }]}, \mathrm{CH}_{\mathrm{n}}^{[\mathrm{OH}]}, \mathrm{OH}$ & $\left(\mathrm{CH}_{2}^{\text {[alc] }}\right)_{2}\left(\mathrm{CH}_{2}^{[\mathrm{fOH}]}\right)_{2}(\mathrm{OH})_{2}$ & 289 & $a_{\mathrm{W}}$ (bulk) & 9 & 0.00 & Ganbavale et al. (2014) \\
\hline 1,4-butanediol & $\mathrm{CH}_{\mathrm{n}}^{[\mathrm{alc}]}, \mathrm{CH}_{\mathrm{n}}^{[\mathrm{OH}]}, \mathrm{OH}$ & $\left(\mathrm{CH}_{2}^{[\mathrm{alcc}]}\right)_{2}\left(\mathrm{CH}_{2}^{[\mathrm{OOH}]}\right)_{2}(\mathrm{OH})_{2}$ & 298 & $a_{\mathrm{W}}$ (bulk) & 9 & 0.00 & Ganbavale et al. (2014) \\
\hline 1,4-butanediol & $\mathrm{CH}_{\mathrm{n}}^{[\text {[alc }]}, \mathrm{CH}_{\mathrm{n}}^{[\mathrm{OH}]}, \mathrm{OH}$ & $\left(\mathrm{CH}_{2}^{[\text {alc }]}\right)_{2}\left(\mathrm{CH}_{2}^{[\mathrm{OH}]}\right)_{2}(\mathrm{OH})_{2}$ & 313 & $a_{\mathrm{W}}$ (bulk) & 9 & 1.00 & Ganbavale et al. (2014) \\
\hline 1,4-butanediol & $\mathrm{CH}_{\mathrm{n}}^{[\mathrm{alc}]}, \mathrm{CH}_{\mathrm{n}}^{[\mathrm{OH}]}, \mathrm{OH}$ & $\left(\mathrm{CH}_{2}^{[\mathrm{alc}]}\right)_{2}\left(\mathrm{CH}_{2}^{[\mathrm{OOH}]}\right)_{2}(\mathrm{OH})_{2}$ & 333 & VLE & 10 & 1.00 & Gmehling and Onken (2003b) \\
\hline 1,4-butanediol & $\mathrm{CH}_{\mathrm{n}}^{[\text {[alc }]}, \mathrm{CH}_{\mathrm{n}}^{[\mathrm{OH}]}, \mathrm{OH}$ & $\left(\mathrm{CH}_{2}^{[\mathrm{alc}]}\right)_{2}\left(\mathrm{CH}_{2}^{[\mathrm{fOH}]}\right)_{2}(\mathrm{OH})_{2}$ & 338 & VLE & 10 & 1.00 & Gmehling and Onken (2003b) \\
\hline 1,4-butanediol & $\mathrm{CH}_{\mathrm{n}}^{[\text {[alc }]}, \mathrm{CH}_{\mathrm{n}}^{[\mathrm{OH}]}, \mathrm{OH}$ & $\left(\mathrm{CH}_{2}^{[\text {[alc] }]}\right)_{2}\left(\mathrm{CH}_{2}^{[\mathrm{OH}]}\right)_{2}(\mathrm{OH})_{2}$ & 343 & VLE & 10 & 1.00 & Gmehling and Onken (2003b) \\
\hline 1,4-butanediol & $\mathrm{CH}_{\mathrm{n}}^{[\mathrm{alc}]}, \mathrm{CH}_{\mathrm{n}}^{[\mathrm{OH}]}, \mathrm{OH}$ & $\left(\mathrm{CH}_{2}^{[\text {alc }}\right)_{2}\left(\mathrm{CH}_{2}^{[\mathrm{OH}]}\right)_{2}(\mathrm{OH})_{2}$ & 348 & VLE & 10 & 1.00 & Gmehling and Onken (2003b) \\
\hline 1,4-butanediol & $\mathrm{CH}_{\mathrm{n}}^{[\mathrm{alc}]}, \mathrm{CH}_{\mathrm{n}}^{[\mathrm{OH}]}, \mathrm{OH}$ & $\left(\mathrm{CH}_{2}^{\text {[alc] }}\right)_{2}\left(\mathrm{CH}_{2}^{[\mathrm{OH}]}\right)_{2}(\mathrm{OH})_{2}$ & 353 & VLE & 9 & 1.00 & Gmehling and Onken (2003b) \\
\hline 1,4-butanediol & $\mathrm{CH}_{\mathrm{n}}^{[\mathrm{alc}]}, \mathrm{CH}_{\mathrm{n}}^{[\mathrm{OH}]}, \mathrm{OH}$ & $\left(\mathrm{CH}_{2}^{[\mathrm{alc}]}\right)_{2}\left(\mathrm{CH}_{2}^{[\mathrm{OH}]}\right)_{2}(\mathrm{OH})_{2}$ & 358 & VLE & 10 & 1.00 & Gmehling and Onken (2003b) \\
\hline 1,4-butanediol & $\mathrm{CH}_{\mathrm{n}}^{[\text {alc }]}, \mathrm{CH}_{\mathrm{n}}^{[\mathrm{OH}]}, \mathrm{OH}$ & $\left(\mathrm{CH}_{2}^{[\text {alc }]}\right)_{2}\left(\mathrm{CH}_{2}^{[\mathrm{fO}]}\right)_{2}(\mathrm{OH})_{2}$ & 363 & VLE & 10 & 1.00 & Gmehling and Onken (2003b) \\
\hline 1,4-butanediol & $\mathrm{CH}_{\mathrm{n}}^{[\text {alc }]}, \mathrm{CH}_{\mathrm{n}}^{[\mathrm{OH}]}, \mathrm{OH}$ & $\left(\mathrm{CH}_{2}^{\text {[alc] }]}\right)_{2}\left(\mathrm{CH}_{2}^{[\mathrm{OH}]}\right)_{2}(\mathrm{OH})_{2}$ & 368 & VLE & 10 & 1.00 & Gmehling and Onken (2003b) \\
\hline 1,4-butanediol & $\mathrm{CH}_{\mathrm{n}}^{[\text {alc }]}, \mathrm{CH}_{\mathrm{n}}^{[\mathrm{OH}]}, \mathrm{OH}$ & $\left(\mathrm{CH}_{2}^{[\text {[alc }]}\right)_{2}\left(\mathrm{CH}_{2}^{[\mathrm{OH}]}\right)_{2}(\mathrm{OH})_{2}$ & $367-409$ & VLE & 13 & 1.00 & Gmehling et al. (1988) \\
\hline 2,3-butanediol & $\mathrm{CH}_{\mathrm{n}}^{[\text {alc }]}, \mathrm{CH}_{\mathrm{n}}^{[\mathrm{OH}]}, \mathrm{OH}$ & $\left(\mathrm{CH}_{3}^{[\text {alc }]}\right)_{2}\left(\mathrm{CH}^{[\mathrm{OH}]}\right)_{2}(\mathrm{OH})_{2}$ & $232-270$ & SLE & 6 & 5.00 & Clendenning (1946) \\
\hline 2,3-butanediol & $\mathrm{CH}_{\mathrm{n}}^{[\mathrm{alc}]}, \mathrm{CH}_{\mathrm{n}}^{[\mathrm{OH}]}, \mathrm{OH}$ & $\left(\mathrm{CH}_{3}^{\text {[alc }]}\right)_{2}\left(\mathrm{CH}^{[\mathrm{OH}]}\right)_{2}(\mathrm{OH})_{2}$ & 298 & $a_{\mathrm{W}}$ (bulk) & 13 & 0.00 & Marcolli and Peter (2005) \\
\hline 2,3-butanediol & $\mathrm{CH}_{\mathrm{n}}^{[\text {[alc }]}, \mathrm{CH}_{\mathrm{n}}^{[\mathrm{OH}]}, \mathrm{OH}$ & $\left(\mathrm{CH}_{3}^{\text {[alc] }}\right)_{2}\left(\mathrm{CH}^{[\mathrm{OH}]}\right)_{2}(\mathrm{OH})_{2}$ & $375-420$ & VLE & 8 & 1.00 & Gmehling et al. (1988) \\
\hline 2,3-butanediol & $\mathrm{CH}_{\mathrm{n}}^{[\mathrm{alc}]}, \mathrm{CH}_{\mathrm{n}}^{[\mathrm{OH}]}, \mathrm{OH}$ & $\left(\mathrm{CH}_{3}^{\text {[alc] }}\right)_{2}\left(\mathrm{CH}^{[\mathrm{OH}]}\right)_{2}(\mathrm{OH})_{2}$ & $373-379$ & VLE & 7 & 1.00 & Gmehling et al. (1988) \\
\hline 2,3-butanediol & $\mathrm{CH}_{\mathrm{n}}^{[\text {alc }]}, \mathrm{CH}_{\mathrm{n}}^{[\mathrm{OH}]}, \mathrm{OH}$ & $\left(\mathrm{CH}_{3}^{[\mathrm{alc}]}\right)_{2}\left(\mathrm{CH}^{[\mathrm{OH}]}\right)_{2}(\mathrm{OH})_{2}$ & $367-411$ & VLE & 8 & 1.00 & Gmehling et al. (1988) \\
\hline 2,3-butanediol & $\mathrm{CH}_{\mathrm{n}}^{[\text {[alc] }]}, \mathrm{CH}_{\mathrm{n}}^{[\mathrm{OH}]}, \mathrm{OH}$ & $\left(\mathrm{CH}_{3}^{\text {[alc }]}\right)_{2}\left(\mathrm{CH}^{[\mathrm{OH}]}\right)_{2}(\mathrm{OH})_{2}$ & 356-399 & VLE & 8 & 1.00 & Gmehling et al. (1988) \\
\hline 2,3-butanediol & $\mathrm{CH}_{\mathrm{n}}^{[\text {alc }]}, \mathrm{CH}_{\mathrm{n}}^{[\mathrm{OH}]}, \mathrm{OH}$ & $\left(\mathrm{CH}_{3}^{\text {[alc] }}\right)_{2}\left(\mathrm{CH}^{[\mathrm{OH}]}\right)_{2}(\mathrm{OH})_{2}$ & $340-380$ & VLE & 8 & 1.00 & Gmehling et al. (1988) \\
\hline 2,3-butanediol & $\mathrm{CH}_{\mathrm{n}}^{[\mathrm{alc}]}, \mathrm{CH}_{\mathrm{n}}^{[\mathrm{OH}]}, \mathrm{OH}$ & $\left(\mathrm{CH}_{3}^{[\mathrm{lalc}]}\right)_{2}\left(\mathrm{CH}^{[\mathrm{OH}]}\right)_{2}(\mathrm{OH})_{2}$ & $408-410$ & VLE & 8 & 1.00 & Gmehling et al. (1988) \\
\hline 2,3-butanediol & $\mathrm{CH}_{\mathrm{n}}^{[\mathrm{alc}]}, \mathrm{CH}_{\mathrm{n}}^{[\mathrm{OH}]}, \mathrm{OH}$ & $\left(\mathrm{CH}_{3}^{\text {[alc }]}\right)_{2}\left(\mathrm{CH}^{[\mathrm{OH}]}\right)_{2}(\mathrm{OH})_{2}$ & $426-431$ & VLE & 7 & 1.00 & Gmehling et al. (1988) \\
\hline 1,5-pentanediol & $\mathrm{CH}_{\mathrm{n}}^{[\text {alc-tail] }]}, \mathrm{CH}_{\mathrm{n}}^{[\mathrm{OH}]}, \mathrm{OH}$ & $\left(\mathrm{CH}_{2}^{\text {[alc-tail] }}\right)_{3}\left(\mathrm{CH}_{2}^{[\mathrm{OH}]}\right)_{2}(\mathrm{OH})_{2}$ & $200-232$ & $a_{\mathrm{w}}\left(T_{\mathrm{hom}}\right)^{\mathrm{a}}$ & 5 & 1.00 & Ganbavale et al. (2014) \\
\hline 1,5-pentanediol & $\mathrm{CH}_{\mathrm{n}}^{[\text {alc-tail] }}, \mathrm{CH}_{\mathrm{n}}^{[\mathrm{OH}]}, \mathrm{OH}$ & $\left(\mathrm{CH}_{2}^{[\text {alc-tail] }}\right)_{3}\left(\mathrm{CH}_{2}^{[\mathrm{OH}]}\right)_{2}(\mathrm{OH})_{2}$ & $260-272$ & SLE & 5 & 5.00 & Ganbavale et al. (2014) \\
\hline
\end{tabular}


Table 1. Continued.

\begin{tabular}{|c|c|c|c|c|c|c|c|}
\hline Organic compounds & Org. main groups & Chemical formula (subgroups) & $T(\mathrm{~K})$ & Data type & $N_{d}$ & $w_{d}^{\text {init }}$ & Reference \\
\hline 1,5-pentanediol & $\begin{array}{l}\mathrm{CH}_{\mathrm{n}}^{[\text {alc-tail }]}, \quad \mathrm{CH}_{\mathrm{n}}^{[\mathrm{OH}]}, \\
\mathrm{OH}\end{array}$ & $\left(\mathrm{CH}_{2}^{[\text {alc-tail }]}\right)_{3}\left(\mathrm{CH}_{2}^{[\mathrm{OH}]}\right)_{2}(\mathrm{OH})_{2}$ & 298 & $a_{\mathrm{W}}$ (bulk) & 14 & 0.00 & Marcolli and Peter (2005) \\
\hline 1,2-hexanediol & $\begin{array}{l}\mathrm{CH}_{\mathrm{n}}^{[\mathrm{alc}-\text { tail }]}, \quad \mathrm{CH}_{\mathrm{n}}^{[\mathrm{OH}]}, \\
\mathrm{OH}\end{array}$ & $\begin{array}{l}\left(\mathrm{CH}_{3}^{[\text {alc-tail }]}\right)\left(\mathrm{CH}_{2}^{[\text {alc-tail }]}\right)_{3} \\
\left(\mathrm{CH}_{2}^{[\mathrm{OH}]}\right)\left(\mathrm{CH}^{[\mathrm{OH}]}\right)(\mathrm{OH})_{2}\end{array}$ & $223-232$ & $a_{\mathrm{w}}\left(T_{\mathrm{hom}}\right)^{\mathrm{a}}$ & 4 & 1.00 & Ganbavale et al. (2014). \\
\hline 1,2-hexanediol & $\begin{array}{l}\mathrm{CH}_{\mathrm{n}}^{[\text {alc-tail }]}, \quad \mathrm{CH}_{\mathrm{n}}^{[\mathrm{OH}]}, \\
\mathrm{OH}\end{array}$ & $\begin{array}{l}\left(\mathrm{CH}_{3}^{[\text {alc-tail }]}\right)\left(\mathrm{CH}_{2}^{[\text {alc-tail }]}\right)_{3} \\
\left(\mathrm{CH}_{2}^{[\mathrm{OH}]}\right)\left(\mathrm{CH}^{[\mathrm{OH}]}\right)(\mathrm{OH})_{2}\end{array}$ & $272-271$ & SLE & 4 & 5.00 & Ganbavale et al. (2014). \\
\hline 1,2-hexanediol & $\begin{array}{l}\mathrm{CH}_{\mathrm{n}}^{[\mathrm{alc}-\mathrm{tail}]}, \quad \mathrm{CH}_{\mathrm{n}}^{[\mathrm{OH}]}, \\
\mathrm{OH}\end{array}$ & $\begin{array}{l}\left(\mathrm{CH}_{3}^{\text {[alc-tail }]}\right)\left(\mathrm{CH}_{2}^{[\text {alc-tail }]}\right)_{3} \\
\left(\mathrm{CH}_{2}^{[\mathrm{OH}]}\right)\left(\mathrm{CH}^{[\mathrm{OH}]}\right)(\mathrm{OH})_{2}\end{array}$ & 298 & $a_{\mathrm{w}}($ bulk) & 12 & 0.00 & Marcolli and Peter (2005) \\
\hline 2,5-hexanediol & $\mathrm{CH}_{\mathrm{n}}^{[\mathrm{alc}]}, \mathrm{CH}_{\mathrm{n}}^{[\mathrm{OH}]}, \mathrm{OH}$ & $\left(\mathrm{CH}_{3}^{[\mathrm{falc}]}\right)_{2}\left(\mathrm{CH}_{2}^{[\mathrm{alc}]}\right)_{2}\left(\mathrm{CH}^{[\mathrm{OH}]}\right)_{2}(\mathrm{OH})_{2}$ & $204-230$ & $a_{\mathrm{w}}\left(T_{\mathrm{hom}}\right)^{\mathrm{a}}$ & 3 & 1.00 & Zobrist et al. (2008) \\
\hline 2,5-hexanediol & $\mathrm{CH}_{\mathrm{n}}^{[\text {[alc }]}, \mathrm{CH}_{\mathrm{n}}^{[\mathrm{OH}]}, \mathrm{OH}$ & $\left(\mathrm{CH}_{3}^{[\text {[alc] }}\right)_{2}\left(\mathrm{CH}_{2}^{[\text {[alc }}\right)_{2}\left(\mathrm{CH}^{[\mathrm{OH}]}\right)_{2}(\mathrm{OH})_{2}$ & $264-271$ & SLE & 3 & 5.00 & Zobrist et al. (2008) \\
\hline 2,5-hexanediol & $\mathrm{CH}_{\mathrm{n}}^{[\mathrm{alc}]}, \mathrm{CH}_{\mathrm{n}}^{[\mathrm{OH}]}, \mathrm{OH}$ & $\left(\mathrm{CH}_{3}^{[\text {alc }]}\right)_{2}\left(\mathrm{CH}_{2}^{[\text {alc }]}\right)_{2}\left(\mathrm{CH}^{[\mathrm{OH}]}\right)_{2}(\mathrm{OH})_{2}$ & 289 & $a_{\mathrm{w}}($ bulk) & 9 & 0.00 & this work \\
\hline 2,5-hexanediol & $\mathrm{CH}_{\mathrm{n}}^{[\text {[alc] }}, \mathrm{CH}_{\mathrm{n}}^{[\mathrm{OH}]}, \mathrm{OH}$ & $\left(\mathrm{CH}_{3}^{\text {[alc] }}\right)_{2}\left(\mathrm{CH}_{2}^{\text {[alc }}\right)_{2}\left(\mathrm{CH}^{[\mathrm{OH}]}\right)_{2}(\mathrm{OH})_{2}$ & 298 & $a_{\mathrm{W}}$ (bulk) & 9 & 0.00 & this work \\
\hline 2,5-hexanediol & $\mathrm{CH}_{\mathrm{n}}^{[\mathrm{alc}]}, \mathrm{CH}_{\mathrm{n}}^{[\mathrm{OH}]}, \mathrm{OH}$ & $\left(\mathrm{CH}_{3}^{[\mathrm{alc}]}\right)_{2}\left(\mathrm{CH}_{2}^{[\mathrm{falc}]}\right)_{2}\left(\mathrm{CH}^{[\mathrm{OH}]}\right)_{2}(\mathrm{OH})_{2}$ & 313 & $a_{\mathrm{W}}$ (bulk) & 9 & 1.00 & this work \\
\hline 1,2,6-hexanetriol & $\mathrm{CH}_{\mathrm{n}}^{[\text {[alc] }}, \mathrm{CH}_{\mathrm{n}}^{[\mathrm{OH}]}, \mathrm{OH}$ & $\left(\mathrm{CH}_{2}^{\text {lalc] }}\right)_{3}\left(\mathrm{CH}_{2}^{[\mathrm{OH}]}\right)_{2}\left(\mathrm{CH}^{[\mathrm{OH}]}\right)(\mathrm{OH})_{3}$ & $202-231$ & $a_{\mathrm{W}}\left(T_{\mathrm{hom}}\right)^{\mathrm{a}}$ & 4 & 1.00 & Zobrist et al. (2008) \\
\hline 1,2,6-hexanetriol & $\mathrm{CH}_{\mathrm{n}}^{[\mathrm{alc}]}, \mathrm{CH}_{\mathrm{n}}^{[\mathrm{OH}]}, \mathrm{OH}$ & $\left(\mathrm{CH}_{2}^{[\mathrm{alc}]}\right)_{3}\left(\mathrm{CH}_{2}^{[\mathrm{OH}]}\right)_{2}\left(\mathrm{CH}^{[\mathrm{OH}]}\right)(\mathrm{OH})_{3}$ & $251-272$ & SLE & 6 & 5.00 & Ross (1954) \\
\hline 1,2,6-hexanetriol & $\mathrm{CH}_{\mathrm{n}}^{[\text {[alc }]}, \mathrm{CH}_{\mathrm{n}}^{[\mathrm{OH}]}, \mathrm{OH}$ & $\left(\mathrm{CH}_{2}^{[\mathrm{alc}]}\right)_{3}\left(\mathrm{CH}_{2}^{[\mathrm{OH}]}\right)_{2}\left(\mathrm{CH}^{[\mathrm{OH}]}\right)(\mathrm{OH})_{3}$ & $263-271$ & SLE & 4 & 5.00 & Zobrist et al. (2008) \\
\hline 1,2,6-hexanetriol & $\mathrm{CH}_{\mathrm{n}}^{[\text {alc }]}, \mathrm{CH}_{\mathrm{n}}^{[\mathrm{OH}]}, \mathrm{OH}$ & $\left(\mathrm{CH}_{2}^{[\mathrm{alc}]}\right)_{3}\left(\mathrm{CH}_{2}^{[\mathrm{OH}]}\right)_{2}\left(\mathrm{CH}^{[\mathrm{OH}]}\right)(\mathrm{OH})_{3}$ & 298 & $a_{\mathrm{W}}$ (bulk) & 9 & 0.00 & this work \\
\hline 1,2,6-hexanetriol & $\mathrm{CH}_{\mathrm{n}}^{[\mathrm{alc}]}, \mathrm{CH}_{\mathrm{n}}^{[\mathrm{OH}]}, \mathrm{OH}$ & $\left(\mathrm{CH}_{2}^{[\mathrm{alc}]}\right)_{3}\left(\mathrm{CH}_{2}^{[\mathrm{OH}]}\right)_{2}\left(\mathrm{CH}^{[\mathrm{OH}]}\right)(\mathrm{OH})_{3}$ & 313 & $a_{\mathrm{w}}$ (bulk) & 9 & 1.00 & this work \\
\hline $1,2,7,8$-octanetetrol & $\mathrm{CH}_{\mathrm{n}}^{[\text {alc }]}, \mathrm{CH}_{\mathrm{n}}^{[\mathrm{OH}]}, \mathrm{OH}$ & $\left(\mathrm{CH}_{2}^{[\mathrm{alc}]}\right)_{4}\left(\mathrm{CH}_{2}^{[\mathrm{OH}]}\right)_{2}\left(\mathrm{CH}^{[\mathrm{OH}]}\right)_{2}(\mathrm{OH})_{4}$ & $203-232$ & $a_{\mathrm{w}}\left(T_{\mathrm{hom}}\right)^{\mathrm{a}}$ & 4 & 1.00 & Zobrist et al. (2008) \\
\hline 1,2,7,8-octanetetrol & $\mathrm{CH}_{\mathrm{n}}^{[\mathrm{alc}]}, \mathrm{CH}_{\mathrm{n}}^{[\mathrm{OH}]}, \mathrm{OH}$ & $\left(\mathrm{CH}_{2}^{[\mathrm{alc}]}\right)_{4}\left(\mathrm{CH}_{2}^{[\mathrm{OH}]}\right)_{2}\left(\mathrm{CH}^{[\mathrm{OH}]}\right)_{2}(\mathrm{OH})_{4}$ & $266-273$ & SLE & 4 & 5.00 & Zobrist et al. (2008) \\
\hline 1,2,7,8-octanetetrol & $\mathrm{CH}_{\mathrm{n}}^{[\mathrm{alc}]}, \mathrm{CH}_{\mathrm{n}}^{[\mathrm{OH}]}, \mathrm{OH}$ & $\left(\mathrm{CH}_{2}^{[\mathrm{alc}]}\right)_{4}\left(\mathrm{CH}_{2}^{[\mathrm{OH}]}\right)_{2}\left(\mathrm{CH}^{[\mathrm{OH}]}\right)_{2}(\mathrm{OH})_{4}$ & 289 & $a_{\mathrm{W}}$ (bulk) & 8 & 0.00 & this work \\
\hline 1,2,7,8-octanetetrol & $\mathrm{CH}_{\mathrm{n}}^{[\text {alc }]}, \mathrm{CH}_{\mathrm{n}}^{[\mathrm{OH}]}, \mathrm{OH}$ & $\left(\mathrm{CH}_{2}^{[\mathrm{alc}]}\right)_{4}\left(\mathrm{CH}_{2}^{[\mathrm{OH}]}\right)_{2}\left(\mathrm{CH}^{[\mathrm{OH}]}\right)_{2}(\mathrm{OH})_{4}$ & 298 & $a_{\mathrm{w}}$ (bulk) & 8 & 0.00 & this work \\
\hline $1,2,7,8$-octanetetrol & $\mathrm{CH}_{\mathrm{n}}^{[\mathrm{alc}]}, \mathrm{CH}_{\mathrm{n}}^{[\mathrm{OH}]}, \mathrm{OH}$ & $\left(\mathrm{CH}_{2}^{[\mathrm{alc}]}\right)_{4}\left(\mathrm{CH}_{2}^{[\mathrm{OH}]}\right)_{2}\left(\mathrm{CH}^{[\mathrm{OH}]}\right)_{2}(\mathrm{OH})_{4}$ & 313 & $a_{\mathrm{W}}$ (bulk) & 9 & 1.00 & this work \\
\hline $\begin{array}{l}\text { 2,2,6,6-tetrakis } \\
\text { (hydroxymethyl)cyclohexanol }\end{array}$ & $\mathrm{CH}_{\mathrm{n}}^{[\mathrm{alc}]}, \mathrm{CH}_{\mathrm{n}}^{[\mathrm{OH}]}, \mathrm{OH}$ & $\begin{array}{l}\left(\mathrm{CH}_{2}^{[\mathrm{alc}]}\right)_{2}\left(\mathrm{CH}^{[\mathrm{alc}]}\right)_{2}\left(\mathrm{CH}_{2}^{[\mathrm{OH}]}\right)_{4} \\
\left(\mathrm{CH}^{[\mathrm{OH}]}\right)\left(\mathrm{C}^{[\mathrm{alc}]}\right)(\mathrm{OH})_{5}\end{array}$ & $208-232$ & $a_{\mathrm{w}}\left(T_{\mathrm{hom}}\right)^{\mathrm{a}}$ & 4 & 1.00 & Zobrist et al. (2008) \\
\hline $\begin{array}{l}\text { 2,2,6,6-tetrakis } \\
\text { (hydroxymethyl)cyclohexanol }\end{array}$ & $\mathrm{CH}_{\mathrm{n}}^{[\mathrm{alc}]}, \mathrm{CH}_{\mathrm{n}}^{[\mathrm{OH}]}, \mathrm{OH}$ & $\begin{array}{l}\left(\mathrm{CH}_{2}^{[\mathrm{alc}]}\right)_{2}\left(\mathrm{CH}^{[\mathrm{alc}]}\right)_{2}\left(\mathrm{CH}_{2}^{[\mathrm{OH}]}\right)_{4} \\
\left(\mathrm{CH}^{[\mathrm{OH}]}\right)\left(\mathrm{C}^{[\mathrm{alc}]}\right)(\mathrm{OH})_{5}\end{array}$ & $265-272$ & SLE & 5 & 5.00 & Zobrist et al. (2008) \\
\hline $\begin{array}{l}\text { 2,2,6,6-tetrakis } \\
\text { (hydroxymethyl)cyclohexanol }\end{array}$ & $\mathrm{CH}_{\mathrm{n}}^{[\mathrm{alc}]}, \mathrm{CH}_{\mathrm{n}}^{[\mathrm{OH}]}, \mathrm{OH}$ & $\begin{array}{l}\left(\mathrm{CH}_{2}^{[\mathrm{alc}]}\right)_{2}\left(\mathrm{CH}^{[\mathrm{alc}]}\right)_{2}\left(\mathrm{CH}_{2}^{[\mathrm{OH}]}\right)_{4} \\
\left(\mathrm{CH}^{[\mathrm{OH}]}\right)\left(\mathrm{C}^{[\mathrm{alc}]}\right)(\mathrm{OH})_{5}\end{array}$ & 289 & $a_{\mathrm{W}}($ bulk) & 8 & 0.00 & this work \\
\hline $\begin{array}{l}\text { 2,2,6,6-tetrakis } \\
\text { (hydroxymethyl)cyclohexanol }\end{array}$ & $\mathrm{CH}_{\mathrm{n}}^{[\mathrm{alc}]}, \mathrm{CH}_{\mathrm{n}}^{[\mathrm{OH}]}, \mathrm{OH}$ & $\begin{array}{l}\left(\mathrm{CH}_{2}^{[\mathrm{alc}]}\right)_{2}\left(\mathrm{CH}^{[\mathrm{alc}]}\right)_{2}\left(\mathrm{CH}_{2}^{[\mathrm{OH}]}\right)_{4} \\
\left(\mathrm{CH}^{[\mathrm{OH}]}\right)\left(\mathrm{C}^{[\mathrm{alc}]}\right)(\mathrm{OH})_{5}\end{array}$ & 298 & $a_{\mathrm{w}}$ (bulk) & 8 & 0.50 & this work \\
\hline $\begin{array}{l}\text { 2,2,6,6-tetrakis } \\
\text { (hydroxymethyl)cyclohexanol }\end{array}$ & $\mathrm{CH}_{\mathrm{n}}^{[\mathrm{alc}]}, \mathrm{CH}_{\mathrm{n}}^{[\mathrm{OH}]}, \mathrm{OH}$ & $\begin{array}{l}\left(\mathrm{CH}_{2}^{[\mathrm{alc}]}\right)_{2}\left(\mathrm{CH}^{[\mathrm{alc}]}\right)_{2}\left(\mathrm{CH}_{2}^{[\mathrm{OH}]}\right)_{4} \\
\left(\mathrm{CH}^{[\mathrm{OH}]}\right)\left(\mathrm{C}^{[\mathrm{alc}]}\right)(\mathrm{OH})_{5}\end{array}$ & 313 & $a_{\mathrm{w}}($ bulk $)$ & 8 & 1.00 & this work \\
\hline sorbitol & $\mathrm{CH}_{\mathrm{n}}^{[\mathrm{OH}]}, \mathrm{OH}$ & $\left(\mathrm{CH}_{2}^{[\mathrm{OH}]}\right)_{2}\left(\mathrm{CH}^{[\mathrm{OH}]}\right)_{4}(\mathrm{OH})_{6}$ & $256-272$ & SLE & 6 & 5.00 & Ganbavale et al. (2014). \\
\hline sorbitol & $\mathrm{CH}_{\mathrm{n}}^{[\mathrm{OH}]}, \mathrm{OH}$ & $\left(\mathrm{CH}_{2}^{[\mathrm{OH}]}\right)_{2}\left(\mathrm{CH}^{[\mathrm{OH}]}\right)_{4}(\mathrm{OH})_{6}$ & 298 & $a_{\mathrm{w}}$ (bulk) & 7 & 0.00 & Ganbavale et al. (2014). \\
\hline sorbitol & $\mathrm{CH}_{\mathrm{n}}^{[\mathrm{OH}]}, \mathrm{OH}$ & $\left(\mathrm{CH}_{2}^{[\mathrm{OH}]}\right)_{2}\left(\mathrm{CH}^{[\mathrm{OH}]}\right)_{4}(\mathrm{OH})_{6}$ & 298 & $a_{\mathrm{W}}$ (bulk) & 8 & 0.00 & Bower and Robinson (1963) \\
\hline sorbitol & $\mathrm{CH}_{\mathrm{n}}^{[\mathrm{OH}]}, \mathrm{OH}$ & $\left(\mathrm{CH}_{2}^{[\mathrm{OH}]}\right)_{2}\left(\mathrm{CH}^{[\mathrm{OH}]}\right)_{4}(\mathrm{OH})_{6}$ & 298 & $a_{\mathrm{W}}$ (bulk) & 8 & 0.00 & Peng et al. (2001) \\
\hline \multicolumn{8}{|c|}{ - water + carboxylic/dicarboxylic acid systems - } \\
\hline acetic acid & $\mathrm{CH}_{\mathrm{n}}, \mathrm{COOH}$ & $\left(\mathrm{CH}_{3}\right)(\mathrm{COOH})$ & $249-272$ & SLE & 12 & 5.00 & Faucon (1910) \\
\hline acetic acid & $\mathrm{CH}_{\mathrm{n}}, \mathrm{COOH}$ & $\left(\mathrm{CH}_{3}\right)(\mathrm{COOH})$ & $250-289$ & SLE & 26 & 5.00 & Pickering (1893) \\
\hline acetic acid & $\mathrm{CH}_{\mathrm{n}}, \mathrm{COOH}$ & $\left(\mathrm{CH}_{3}\right)(\mathrm{COOH})$ & $249-290$ & SLE(org) ${ }^{\mathrm{d}}$ & 11 & 0.20 & Faucon $(1910)$ \\
\hline acetic acid & $\mathrm{CH}_{\mathrm{n}}, \mathrm{COOH}$ & $\left(\mathrm{CH}_{3}\right)(\mathrm{COOH})$ & $251-273$ & SLE(org) ${ }^{\mathrm{d}}$ & 20 & 0.20 & Pickering (1893) \\
\hline acetic acid & $\mathrm{CH}_{\mathrm{n}}, \mathrm{COOH}$ & $\left(\mathrm{CH}_{3}\right)(\mathrm{COOH})$ & 298 & VLE & 8 & 0.00 & Campbell et al. (1963) \\
\hline acetic acid & $\mathrm{CH}_{\mathrm{n}}, \mathrm{COOH}$ & $\left(\mathrm{CH}_{3}\right)(\mathrm{COOH})$ & 374-389 & VLE & 10 & 0.20 & Sebastiani and Lacquaniti (1967) \\
\hline acetic acid & $\mathrm{CH}_{\mathrm{n}}, \mathrm{COOH}$ & $\left(\mathrm{CH}_{3}\right)(\mathrm{COOH})$ & $373-390$ & VLE & 16 & 0.20 & Ito and Yoshida (1963) \\
\hline acetic acid & $\mathrm{CH}_{\mathrm{n}}, \mathrm{COOH}$ & $\left(\mathrm{CH}_{3}\right)(\mathrm{COOH})$ & $340-351$ & VLE & 15 & 0.20 & Ito and Yoshida (1963) \\
\hline acetic acid & $\mathrm{CH}_{\mathrm{n}}, \mathrm{COOH}$ & $\left(\mathrm{CH}_{3}\right)(\mathrm{COOH})$ & $318-326$ & VLE & 14 & 0.20 & Ito and Yoshida (1963) \\
\hline acetic acid & $\mathrm{CH}_{\mathrm{n}}, \mathrm{COOH}$ & $\left(\mathrm{CH}_{3}\right)(\mathrm{COOH})$ & 343 & VLE & 11 & 0.20 & Arich and Tagliavini (1958) \\
\hline acetic acid & $\mathrm{CH}_{\mathrm{n}}, \mathrm{COOH}$ & $\left(\mathrm{CH}_{3}\right)(\mathrm{COOH})$ & 353 & VLE & 11 & 0.20 & Arich and Tagliavini (1958) \\
\hline acetic acid & $\mathrm{CH}_{\mathrm{n}}, \mathrm{COOH}$ & $\left(\mathrm{CH}_{3}\right)(\mathrm{COOH})$ & 363 & VLE & 11 & 0.20 & Arich and Tagliavini (1958) \\
\hline acetic acid & $\mathrm{CH}_{\mathrm{n}}, \mathrm{COOH}$ & $\left(\mathrm{CH}_{3}\right)(\mathrm{COOH})$ & $322-329$ & VLE & 8 & 0.20 & Keyes (1933) \\
\hline acetic acid & $\mathrm{CH}_{\mathrm{n}}, \mathrm{COOH}$ & $\left(\mathrm{CH}_{3}\right)(\mathrm{COOH})$ & $337-342$ & VLE & 8 & 0.20 & Keyes (1933) \\
\hline acetic acid & $\mathrm{CH}_{\mathrm{n}}, \mathrm{COOH}$ & $\left(\mathrm{CH}_{3}\right)(\mathrm{COOH})$ & $350-355$ & VLE & 8 & 0.20 & Keyes (1933) \\
\hline acetic acid & $\mathrm{CH}_{\mathrm{n}}, \mathrm{COOH}$ & $\left(\mathrm{CH}_{3}\right)(\mathrm{COOH})$ & $373-386$ & VLE & 9 & 0.20 & Narayana et al. (1985) \\
\hline propanoic acid & $\mathrm{CH}_{\mathrm{n}}, \mathrm{COOH}$ & $\left(\mathrm{CH}_{3}\right)\left(\mathrm{CH}_{2}\right)(\mathrm{COOH})$ & $245-273$ & SLE & 19 & 5.00 & Faucon (1910) \\
\hline propanoic acid & $\mathrm{CH}_{\mathrm{n}}, \mathrm{COOH}$ & $\left(\mathrm{CH}_{3}\right)\left(\mathrm{CH}_{2}\right)(\mathrm{COOH})$ & $244-254$ & SLE(org) ${ }^{\mathrm{d}}$ & 8 & 0.20 & Faucon (1910) \\
\hline propanoic acid & $\mathrm{CH}_{\mathrm{n}}, \mathrm{COOH}$ & $\left(\mathrm{CH}_{3}\right)\left(\mathrm{CH}_{2}\right)(\mathrm{COOH})$ & $373-405$ & VLE & 8 & 0.20 & Ito and Yoshida (1963) \\
\hline propanoic acid & $\mathrm{CH}_{\mathrm{n}}, \mathrm{COOH}$ & $\left(\mathrm{CH}_{3}\right)\left(\mathrm{CH}_{2}\right)(\mathrm{COOH})$ & $340-368$ & VLE & 9 & 0.20 & Ito and Yoshida (1963) \\
\hline propanoic acid & $\mathrm{CH}_{\mathrm{n}}, \mathrm{COOH}$ & $\left(\mathrm{CH}_{3}\right)\left(\mathrm{CH}_{2}\right)(\mathrm{COOH})$ & $318-345$ & VLE & 7 & 0.01 & Ito and Yoshida (1963) \\
\hline propanoic acid & $\mathrm{CH}_{\mathrm{n}}, \mathrm{COOH}$ & $\left(\mathrm{CH}_{3}\right)\left(\mathrm{CH}_{2}\right)(\mathrm{COOH})$ & $372-401$ & VLE & 18 & 0.20 & Dakshinamurty et al. (1961) \\
\hline propanoic acid & $\mathrm{CH}_{\mathrm{n}}, \mathrm{COOH}$ & $\left(\mathrm{CH}_{3}\right)\left(\mathrm{CH}_{2}\right)(\mathrm{COOH})$ & $325-354$ & VLE & 24 & 0.2 & Gmehling and Onken (1977) \\
\hline propanoic acid & $\mathrm{CH}_{\mathrm{n}}, \mathrm{COOH}$ & $\left(\mathrm{CH}_{3}\right)\left(\mathrm{CH}_{2}\right)(\mathrm{COOH})$ & $339-373$ & VLE & 25 & 0.2 & Gmehling and Onken (1977) \\
\hline propanoic acid & $\mathrm{CH}_{\mathrm{n}}, \mathrm{COOH}$ & $\left(\mathrm{CH}_{3}\right)\left(\mathrm{CH}_{2}\right)(\mathrm{COOH})$ & $356-392$ & VLE & 23 & 0.20 & Gmehling and Onken (1977) \\
\hline propanoic acid & $\mathrm{CH}_{\mathrm{n}}, \mathrm{COOH}$ & $\left(\mathrm{CH}_{3}\right)\left(\mathrm{CH}_{2}\right)(\mathrm{COOH})$ & $373-411$ & VLE & 24 & 0.20 & Gmehling and Onken (1977) \\
\hline propanoic acid & $\mathrm{CH}_{\mathrm{n}}, \mathrm{COOH}$ & $\left(\mathrm{CH}_{3}\right)\left(\mathrm{CH}_{2}\right)(\mathrm{COOH})$ & $373-411$ & VLE & 12 & 0.01 & Gmehling and Onken (2003a) \\
\hline butanoic acid & $\mathrm{CH}_{\mathrm{n}}, \mathrm{COOH}$ & $\left(\mathrm{CH}_{3}\right)\left(\mathrm{CH}_{2}\right)_{2}(\mathrm{COOH})$ & $261-273$ & SLE & 19 & 5.00 & Faucon $(1910)$ \\
\hline butanoic acid & $\mathrm{CH}_{\mathrm{n}}, \mathrm{COOH}$ & $\left(\mathrm{CH}_{3}\right)\left(\mathrm{CH}_{2}\right)_{2}(\mathrm{COOH})$ & $261-269$ & SLE(org) ${ }^{\mathrm{d}}$ & 8 & 0.20 & Faucon $(1910)$ \\
\hline butanoic acid & $\mathrm{CH}_{\mathrm{n}}, \mathrm{COOH}$ & $\left(\mathrm{CH}_{3}\right)\left(\mathrm{CH}_{2}\right)_{2}(\mathrm{COOH})$ & 303 & VLE & 7 & 0.00 & Wright and Akhtar (1970) \\
\hline butanoic acid & $\mathrm{CH}_{\mathrm{n}}, \mathrm{COOH}$ & $\left(\mathrm{CH}_{3}\right)\left(\mathrm{CH}_{2}\right)_{2}(\mathrm{COOH})$ & 373-394 & VLE & 8 & 1.00 & Gmehling and Onken (1977) \\
\hline
\end{tabular}


Table 1. Continued.

\begin{tabular}{|c|c|c|c|c|c|c|c|}
\hline Organic compounds & Org. main groups & Chemical formula (subgroups) & $T(\mathrm{~K})$ & Data type & $N_{d}$ & $w_{d}^{\text {init }}$ & Reference \\
\hline oxalic acid & $\mathrm{COOH}$ & $(\mathrm{COOH})_{2}$ & $272-273$ & SLE & 4 & 5.00 & Braban et al. (2003) \\
\hline oxalic acid & $\mathrm{COOH}$ & $(\mathrm{COOH})_{2}$ & $277-308$ & $\operatorname{SLE}(\text { org })^{\mathrm{d}}$ & 11 & 0.00 & Braban et al. (2003) \\
\hline oxalic acid & $\mathrm{COOH}$ & $(\mathrm{COOH})_{2}$ & $278-338$ & $\operatorname{SLE}(\text { org })^{\mathrm{d}}$ & 13 & 0.00 & Apelblat and Manzurola (1987) \\
\hline oxalic acid & $\mathrm{COOH}$ & $(\mathrm{COOH})_{2}$ & 298 & $a_{\mathrm{W}}$ & 14 & 0.00 & Peng et al. (2001) \\
\hline oxalic acid & $\mathrm{COOH}$ & $(\mathrm{COOH})_{2}$ & $284-352$ & SLE(org) ${ }^{\mathrm{d}}$ & 8 & 0.00 & Omar and Ulrich (2006) \\
\hline malic acid & $\mathrm{CH}_{\mathrm{n}}, \mathrm{CH}_{\mathrm{n}}^{[\mathrm{OH}]}, \mathrm{COOH}, \mathrm{OH}$ & $\left(\mathrm{CH}_{2}\right)\left(\mathrm{CH}^{[\mathrm{OH}]}\right)(\mathrm{COOH})_{2}(\mathrm{OH})$ & $278-338$ & $\operatorname{SLE}(\text { org })^{\mathrm{d}}$ & 13 & 0.2 & Apelblat and Manzurola (1987) \\
\hline malic acid & $\mathrm{CH}_{\mathrm{n}}, \mathrm{CH}_{\mathrm{n}}^{[\mathrm{OH}]}, \mathrm{COOH}, \mathrm{OH}$ & $\left(\mathrm{CH}_{2}\right)\left(\mathrm{CH}^{[\mathrm{OH}]}\right)(\mathrm{COOH})_{2}(\mathrm{OH})$ & $262-273$ & SLE & 16 & 2.00 & Beyer et al. (2008) \\
\hline malonic acid & $\mathrm{CH}_{\mathrm{n}}, \mathrm{COOH}$ & $\left(\mathrm{CH}_{2}\right)(\mathrm{COOH})_{2}$ & $262-273$ & SLE & 22 & 5.00 & Braban et al. (2003) \\
\hline malonic acid & $\mathrm{CH}_{\mathrm{n}}, \mathrm{COOH}$ & $\left(\mathrm{CH}_{2}\right)(\mathrm{COOH})_{2}$ & $278-338$ & $\operatorname{SLE}(\text { org })^{\mathrm{d}}$ & 13 & 0.2 & Apelblat and Manzurola (1987) \\
\hline malonic acid & $\mathrm{CH}_{\mathrm{n}}, \mathrm{COOH}$ & $\left(\mathrm{CH}_{2}\right)(\mathrm{COOH})_{2}$ & 298 & $a_{\mathrm{W}}$ & 6 & 0.00 & Peng et al. (2001) \\
\hline malonic acid & $\mathrm{CH}_{\mathrm{n}}, \mathrm{COOH}$ & $\left(\mathrm{CH}_{2}\right)(\mathrm{COOH})_{2}$ & 298 & $a_{\mathrm{W}}$ & 6 & 0.00 & Maffia and Meirelles (2001) \\
\hline malonic acid & $\mathrm{CH}_{\mathrm{n}}, \mathrm{COOH}$ & $\left(\mathrm{CH}_{2}\right)(\mathrm{COOH})_{2}$ & 298 & $a_{\mathrm{W}}$ & 7 & 0.00 & Peng et al. (2001) \\
\hline succinic acid & $\mathrm{CH}_{\mathrm{n}}, \mathrm{COOH}$ & $\left(\mathrm{CH}_{2}\right)_{2}(\mathrm{COOH})_{2}$ & 273 & SLE & 9 & 5.00 & Beyer et al. (2008) \\
\hline succinic acid & $\mathrm{CH}_{\mathrm{n}}, \mathrm{COOH}$ & $\left(\mathrm{CH}_{2}\right)_{2}(\mathrm{COOH})_{2}$ & $296-447$ & SLE $(\text { org })^{\mathrm{d}}$ & 10 & 0.20 & Lin et al. (2007) \\
\hline succinic acid & $\mathrm{CH}_{\mathrm{n}}, \mathrm{COOH}$ & $\left(\mathrm{CH}_{2}\right)_{2}(\mathrm{COOH})_{2}$ & $278-338$ & $\operatorname{SLE}(\text { org })^{\mathrm{d}}$ & 13 & 0.20 & Apelblat and Manzurola (1987) \\
\hline succinic acid & $\mathrm{CH}_{\mathrm{n}}, \mathrm{COOH}$ & $\left(\mathrm{CH}_{2}\right)_{2}(\mathrm{COOH})_{2}$ & 298 & $a_{\mathrm{W}}$ & 5 & 0.00 & Maffia and Meirelles (2001) \\
\hline glutaric acid & $\mathrm{CH}_{\mathrm{n}}, \mathrm{COOH}$ & $\left(\mathrm{CH}_{2}\right)_{3}(\mathrm{COOH})_{2}$ & $271-273$ & SLE & 5 & 5.00 & Beyer et al. (2008) \\
\hline glutaric acid & $\mathrm{CH}_{\mathrm{n}}, \mathrm{COOH}$ & $\left(\mathrm{CH}_{2}\right)_{3}(\mathrm{COOH})_{2}$ & $279-336$ & SLE(org) ${ }^{\mathrm{d}}$ & 24 & 0.10 & Apelblat and Manzurola (1989) \\
\hline glutaric acid & $\mathrm{CH}_{\mathrm{n}}, \mathrm{COOH}$ & $\left(\mathrm{CH}_{2}\right)_{3}(\mathrm{COOH})_{2}$ & $277-298$ & $\operatorname{SLE}(\text { org })^{\mathrm{d}}$ & 23 & 0.10 & Beyer et al. (2008) \\
\hline glutaric acid & $\mathrm{CH}_{\mathrm{n}}, \mathrm{COOH}$ & $\left(\mathrm{CH}_{2}\right)_{3}(\mathrm{COOH})_{2}$ & 298 & $a_{\mathrm{W}}$ & 34 & 0.00 & Peng et al. (2001) \\
\hline glutaric acid & $\mathrm{CH}_{\mathrm{n}}, \mathrm{COOH}$ & $\left(\mathrm{CH}_{2}\right)_{3}(\mathrm{COOH})_{2}$ & 291 & $a_{\mathrm{W}}$ & 57 & 0.00 & Zardini et al. (2008) \\
\hline citric acid & $\mathrm{CH}_{\mathrm{n}}, \mathrm{C}^{[\mathrm{OH}]}, \mathrm{COOH}, \mathrm{OH}$ & $\left(\mathrm{CH}_{2}\right)_{2}\left(\mathrm{C}^{[\mathrm{OH}]}\right)(\mathrm{COOH})_{3}(\mathrm{OH})$ & $278-338$ & SLE(org) ${ }^{\mathrm{d}}$ & 13 & 0.0 & Apelblat and Manzurola (1987) \\
\hline citric acid & $\mathrm{CH}_{\mathrm{n}}, \mathrm{C}^{[\mathrm{OH}]}, \mathrm{COOH}, \mathrm{OH}$ & $\left(\mathrm{CH}_{2}\right)_{2}\left(\mathrm{C}^{[\mathrm{OH}]}\right)(\mathrm{COOH})_{3}(\mathrm{OH})$ & 291 & $a_{\mathrm{W}}$ & 90 & 0.00 & Zardini et al. (2008) \\
\hline citric acid & $\mathrm{CH}_{\mathrm{n}}, \mathrm{C}^{[\mathrm{OH}]}, \mathrm{COOH}, \mathrm{OH}$ & $\left(\mathrm{CH}_{2}\right)_{2}\left(\mathrm{C}^{[\mathrm{OH}]}\right)(\mathrm{COOH})_{3}(\mathrm{OH})$ & 298 & $a_{\mathrm{W}}$ & 25 & 0.00 & Peng et al. (2001) \\
\hline adipic acid & $\mathrm{CH}_{\mathrm{n}}, \mathrm{COOH}$ & $\left(\mathrm{CH}_{2}\right)_{4}(\mathrm{COOH})_{2}$ & $278-338$ & SLE(org) $)^{\mathrm{d}}$ & 13 & 0.20 & Apelblat and Manzurola (1987) \\
\hline pimelic acid & $\mathrm{CH}_{\mathrm{n}}, \mathrm{COOH}$ & $\left(\mathrm{CH}_{2}\right)_{5}(\mathrm{COOH})_{2}$ & $279-342$ & $\operatorname{SLE}(\text { org })^{\mathrm{d}}$ & 21 & 0.20 & Apelblat and Manzurola (1989) \\
\hline $\mathrm{M} 5^{\mathrm{b}}$ & $\mathrm{CH}_{\mathrm{n}}, \mathrm{CH}_{\mathrm{n}}^{[\mathrm{OH}]}, \mathrm{COOH}, \mathrm{OH}, \mathrm{C}=\mathrm{C}$ & $\mathrm{c}$ & $191-230$ & $a_{\mathrm{W}}\left(T_{\mathrm{hom}}\right)^{\mathrm{a}}$ & 6 & 1.00 & Zobrist et al. (2008) \\
\hline M5 ${ }^{\mathrm{b}}$ & $\mathrm{CH}_{\mathrm{n}}, \mathrm{CH}_{\mathrm{n}}^{[\mathrm{OH}]}, \mathrm{COOH}, \mathrm{OH}, \mathrm{C}=\mathrm{C}$ & c & $255-271$ & SLE & 6 & 5.00 & Zobrist et al. (2008) \\
\hline M5 $5^{b}$ & $\mathrm{CH}_{\mathrm{n}}, \mathrm{CH}_{\mathrm{n}}^{[\mathrm{OH}]}, \mathrm{COOH}, \mathrm{OH}, \mathrm{C}=\mathrm{C}$ & $\mathrm{c}$ & 233 & $a_{\mathrm{W}}(\mathrm{EDB})^{\mathrm{f}}$ & 3 & 0.20 & Ganbavale et al. (2014) \\
\hline $\mathrm{M}^{\mathrm{b}}$ & $\mathrm{CH}_{\mathrm{n}}, \mathrm{CH}_{\mathrm{n}}^{[\mathrm{OH}]}, \mathrm{COOH}, \mathrm{OH}, \mathrm{C}=\mathrm{C}$ & c & 236 & $a_{\mathrm{W}}(\mathrm{EDB})^{\mathrm{f}}$ & 2 & 0.20 & Ganbavale et al. (2014) \\
\hline M5 $5^{\mathrm{b}}$ & $\mathrm{CH}_{\mathrm{n}}, \mathrm{CH}_{\mathrm{n}}^{[\mathrm{OH}]}, \mathrm{COOH}, \mathrm{OH}, \mathrm{C}=\mathrm{C}$ & $\mathrm{c}$ & 244 & $a_{\mathrm{W}}(\mathrm{EDB})^{\mathrm{f}}$ & 6 & 0.50 & Ganbavale et al. (2014) \\
\hline M5 $5^{\mathrm{b}}$ & $\mathrm{CH}_{\mathrm{n}}, \mathrm{CH}_{\mathrm{n}}^{[\mathrm{OH}]}, \mathrm{COOH}, \mathrm{OH}, \mathrm{C}=\mathrm{C}$ & $\mathrm{c}$ & 253 & $a_{\mathrm{W}}(\mathrm{EDB})^{\mathrm{f}}$ & 3 & 0.50 & Ganbavale et al. (2014) \\
\hline $\mathrm{M}^{\mathrm{b}}$ & $\mathrm{CH}_{\mathrm{n}}, \mathrm{CH}_{\mathrm{n}}^{[\mathrm{OH}]}, \mathrm{COOH}, \mathrm{OH}, \mathrm{C}=\mathrm{C}$ & c & 253 & $a_{\mathrm{W}}\left(p^{\mathrm{tot}}\right)$ & 5 & 1.00 & Ganbavale et al. (2014) \\
\hline $\mathrm{M}^{\mathrm{b}}$ & $\mathrm{CH}_{\mathrm{n}}, \mathrm{CH}_{\mathrm{n}}^{[\mathrm{OH}]}, \mathrm{COOH}, \mathrm{OH}, \mathrm{C}=\mathrm{C}$ & c & 260 & $a_{\mathrm{W}}\left(p^{\mathrm{tot}}\right)$ & 4 & 1.00 & Ganbavale et al. (2014) \\
\hline $\mathrm{M} 5^{\mathrm{b}}$ & $\mathrm{CH}_{\mathrm{n}}, \mathrm{CH}_{\mathrm{n}}^{[\mathrm{OH}]}, \mathrm{COOH}, \mathrm{OH}, \mathrm{C}=\mathrm{C}$ & $\mathrm{c}$ & 263 & $a_{\mathrm{W}}(\mathrm{EDB})^{\mathrm{f}}$ & 2 & 1.00 & Ganbavale et al. (2014) \\
\hline $\mathrm{M}^{\mathrm{b}}$ & $\mathrm{CH}_{\mathrm{n}}, \mathrm{CH}_{\mathrm{n}}^{[\mathrm{OH}]}, \mathrm{COOH}, \mathrm{OH}, \mathrm{C}=\mathrm{C}$ & $\mathrm{c}$ & 263 & $a_{\mathrm{W}}(\mathrm{EDB})^{\mathrm{f}}$ & 5 & 1.00 & Ganbavale et al. (2014) \\
\hline $\mathrm{M} 5^{\mathrm{b}}$ & $\mathrm{CH}_{\mathrm{n}}, \mathrm{CH}_{\mathrm{n}}^{[\mathrm{OH}]}, \mathrm{COOH}, \mathrm{OH}, \mathrm{C}=\mathrm{C}$ & c & 265 & $a_{\mathrm{W}}\left(p^{\mathrm{tot}}\right)$ & 3 & 1.00 & Ganbavale et al. (2014) \\
\hline M5 ${ }^{\mathrm{b}}$ & $\mathrm{CH}_{\mathrm{n}}, \mathrm{CH}_{\mathrm{n}}^{[\mathrm{OH}]}, \mathrm{COOH}, \mathrm{OH}, \mathrm{C}=\mathrm{C}$ & c & 268 & $a_{\mathrm{W}}\left(p^{\mathrm{tot}}\right)$ & 3 & 1.00 & Ganbavale et al. (2014) \\
\hline M5 $5^{\mathrm{b}}$ & $\mathrm{CH}_{\mathrm{n}}, \mathrm{CH}_{\mathrm{n}}^{[\mathrm{OH}]}, \mathrm{COOH}, \mathrm{OH}, \mathrm{C}=\mathrm{C}$ & c & 268 & $a_{\mathrm{W}}(\mathrm{EDB})^{\mathrm{f}}$ & 8 & 1.00 & Ganbavale et al. (2014) \\
\hline M5 $5^{\mathrm{b}}$ & $\mathrm{CH}_{\mathrm{n}}, \mathrm{CH}_{\mathrm{n}}^{[\mathrm{OH}]}, \mathrm{COOH}, \mathrm{OH}, \mathrm{C}=\mathrm{C}$ & $\mathrm{c}$ & 270 & $a_{\mathrm{W}}\left(p^{\mathrm{tot}}\right)$ & 3 & 1.00 & Ganbavale et al. (2014) \\
\hline $\mathrm{M} 5^{\mathrm{b}}$ & $\mathrm{CH}_{\mathrm{n}}, \mathrm{CH}_{\mathrm{n}}^{[\mathrm{OH}]}, \mathrm{COOH}, \mathrm{OH}, \mathrm{C}=\mathrm{C}$ & $\mathrm{c}$ & 273 & $a_{\mathrm{W}}\left(p^{\mathrm{tot}}\right)$ & 3 & 1.00 & Ganbavale et al. (2014) \\
\hline M5 $5^{\mathrm{b}}$ & $\mathrm{CH}_{\mathrm{n}}, \mathrm{CH}_{\mathrm{n}}^{[\mathrm{OH}]}, \mathrm{COOH}, \mathrm{OH}, \mathrm{C}=\mathrm{C}$ & c & 273 & $a_{\mathrm{W}}(\mathrm{EDB})^{\mathrm{f}}$ & 10 & 1.00 & Ganbavale et al. (2014) \\
\hline $\mathrm{M} 5^{\mathrm{b}}$ & $\mathrm{CH}_{\mathrm{n}}, \mathrm{CH}_{\mathrm{n}}^{[\mathrm{OH}]}, \mathrm{COOH}, \mathrm{OH}, \mathrm{C}=\mathrm{C}$ & $\mathrm{c}$ & 275 & $a_{\mathrm{W}}\left(p^{\mathrm{tot}}\right)$ & 3 & 1.00 & Ganbavale et al. (2014) \\
\hline $\mathrm{M}^{\mathrm{b}}$ & $\mathrm{CH}_{\mathrm{n}}, \mathrm{CH}_{\mathrm{n}}^{[\mathrm{OH}]}, \mathrm{COOH}, \mathrm{OH}, \mathrm{C}=\mathrm{C}$ & c & 278 & $a_{\mathrm{W}}\left(p^{\mathrm{tot}}\right)$ & 5 & 1.00 & Ganbavale et al. (2014) \\
\hline M5 $5^{\mathrm{b}}$ & $\mathrm{CH}_{\mathrm{n}}, \mathrm{CH}_{\mathrm{n}}^{[\mathrm{OH}]}, \mathrm{COOH}, \mathrm{OH}, \mathrm{C}=\mathrm{C}$ & $\mathrm{c}$ & 279 & $a_{\mathrm{W}}($ bulk) & 9 & 1.00 & Ganbavale et al. (2014) \\
\hline $\mathrm{M} 5^{\mathrm{b}}$ & $\mathrm{CH}_{\mathrm{n}}, \mathrm{CH}_{\mathrm{n}}^{[\mathrm{OH}]}, \mathrm{COOH}, \mathrm{OH}, \mathrm{C}=\mathrm{C}$ & c & 280 & $a_{\mathrm{W}}\left(p^{\mathrm{tot}}\right)$ & 5 & 1.00 & Ganbavale et al. (2014) \\
\hline $\mathrm{M} 5^{\mathrm{b}}$ & $\mathrm{CH}_{\mathrm{n}}, \mathrm{CH}_{\mathrm{n}}^{[\mathrm{OH}]}, \mathrm{COOH}, \mathrm{OH}, \mathrm{C}=\mathrm{C}$ & c & 283 & $a_{\mathrm{w}}\left(p^{\mathrm{tot}}\right)$ & 5 & 1.00 & Ganbavale et al. (2014) \\
\hline M5 $5^{\mathrm{b}}$ & $\mathrm{CH}_{\mathrm{n}}, \mathrm{CH}_{\mathrm{n}}^{[\mathrm{OH}]}, \mathrm{COOH}, \mathrm{OH}, \mathrm{C}=\mathrm{C}$ & $\mathrm{c}$ & 285 & $a_{\mathrm{W}}\left(p^{\mathrm{tot}}\right)$ & 5 & 1.00 & Ganbavale et al. (2014) \\
\hline M5 $5^{\mathrm{b}}$ & $\mathrm{CH}_{\mathrm{n}}, \mathrm{CH}_{\mathrm{n}}^{[\mathrm{OH}]}, \mathrm{COOH}, \mathrm{OH}, \mathrm{C}=\mathrm{C}$ & $\mathrm{c}$ & 288 & $a_{\mathrm{w}}\left(p^{\mathrm{tot}}\right)$ & 5 & 1.00 & Ganbavale et al. (2014) \\
\hline M5 $5^{\mathrm{b}}$ & $\mathrm{CH}_{\mathrm{n}}, \mathrm{CH}_{\mathrm{n}}^{[\mathrm{OH}]}, \mathrm{COOH}, \mathrm{OH}, \mathrm{C}=\mathrm{C}$ & c & 289 & $a_{\mathrm{W}}(\mathrm{EDB})^{\mathrm{f}}$ & 10 & 0.00 & Ganbavale et al. (2014) \\
\hline M5 $5^{\mathrm{b}}$ & $\mathrm{CH}_{\mathrm{n}}, \mathrm{CH}_{\mathrm{n}}^{[\mathrm{OH}]}, \mathrm{COOH}, \mathrm{OH}, \mathrm{C}=\mathrm{C}$ & $\mathrm{c}$ & 289 & $a_{\mathrm{W}}$ (bulk) & 10 & 0.00 & Ganbavale et al. (2014) \\
\hline $\mathrm{M}^{\mathrm{b}}$ & $\mathrm{CH}_{\mathrm{n}}, \mathrm{CH}_{\mathrm{n}}^{[\mathrm{OH}]}, \mathrm{COOH}, \mathrm{OH}, \mathrm{C}=\mathrm{C}$ & c & 290 & $a_{\mathrm{W}}\left(p^{\mathrm{tot}}\right)$ & 5 & 0.00 & Ganbavale et al. (2014) \\
\hline $\mathrm{M} 5^{\mathrm{b}}$ & $\mathrm{CH}_{\mathrm{n}}, \mathrm{CH}_{\mathrm{n}}^{[\mathrm{OH}]}, \mathrm{COOH}, \mathrm{OH}, \mathrm{C}=\mathrm{C}$ & c & 298 & $a_{\mathrm{W}}$ (bulk) & 10 & 0.00 & Ganbavale et al. (2014) \\
\hline $\mathrm{M} 5^{\mathrm{b}}$ & $\mathrm{CH}_{\mathrm{n}}, \mathrm{CH}_{\mathrm{n}}^{[\mathrm{OH}]}, \mathrm{COOH}, \mathrm{OH}, \mathrm{C}=\mathrm{C}$ & $\mathrm{c}$ & 313 & $a_{\mathrm{W}}($ bulk) & 10 & 0.00 & Ganbavale et al. (2014) \\
\hline \multicolumn{8}{|c|}{ - water + ketone systems - } \\
\hline acetone & $\mathrm{CH}_{\mathrm{n}}, \mathrm{CH}_{\mathrm{n}} \mathrm{CO}$ & $\left(\mathrm{CH}_{3}\right)\left(\mathrm{CH}_{3} \mathrm{CO}\right)$ & $221-273$ & SLE & 17 & 5.00 & Jakob (1994) \\
\hline acetone & $\mathrm{CH}_{\mathrm{n}}, \mathrm{CH}_{\mathrm{n}} \mathrm{CO}$ & $\left(\mathrm{CH}_{3}\right)\left(\mathrm{CH}_{3} \mathrm{CO}\right)$ & 298 & VLE & 13 & 0.00 & Gmehling et al. (1988) \\
\hline acetone & $\mathrm{CH}_{\mathrm{n}}, \mathrm{CH}_{\mathrm{n}} \mathrm{CO}$ & $\left(\mathrm{CH}_{3}\right)\left(\mathrm{CH}_{3} \mathrm{CO}\right)$ & 308 & VLE & 13 & 1.00 & Gmehling et al. (1988) \\
\hline acetone & $\mathrm{CH}_{\mathrm{n}}, \mathrm{CH}_{\mathrm{n}} \mathrm{CO}$ & $\left(\mathrm{CH}_{3}\right)\left(\mathrm{CH}_{3} \mathrm{CO}\right)$ & 318 & VLE & 13 & 1.00 & Gmehling et al. (1988) \\
\hline acetone & $\mathrm{CH}_{\mathrm{n}}, \mathrm{CH}_{\mathrm{n}} \mathrm{CO}$ & $\left(\mathrm{CH}_{3}\right)\left(\mathrm{CH}_{3} \mathrm{CO}\right)$ & 323 & VLE & 13 & 1.00 & Gmehling et al. (1988) \\
\hline acetone & $\mathrm{CH}_{\mathrm{n}}, \mathrm{CH}_{\mathrm{n}} \mathrm{CO}$ & $\left(\mathrm{CH}_{3}\right)\left(\mathrm{CH}_{3} \mathrm{CO}\right)$ & 328 & VLE & 13 & 1.00 & Gmehling et al. (1988) \\
\hline acetone & $\mathrm{CH}_{\mathrm{n}}, \mathrm{CH}_{\mathrm{n}} \mathrm{CO}$ & $\left(\mathrm{CH}_{3}\right)\left(\mathrm{CH}_{3} \mathrm{CO}\right)$ & 373 & VLE & 20 & 1.00 & Griswold and Wong (1952) \\
\hline acetone & $\mathrm{CH}_{\mathrm{n}}, \mathrm{CH}_{\mathrm{n}} \mathrm{CO}$ & $\left(\mathrm{CH}_{3}\right)\left(\mathrm{CH}_{3} \mathrm{CO}\right)$ & $295-321$ & VLE & 10 & 1.00 & Othmer and Benenati (1945) \\
\hline
\end{tabular}


Table 1. Continued.

\begin{tabular}{|c|c|c|c|c|c|c|c|}
\hline Organic compounds & Org. main groups & Chemical formula (subgroups) & $T(\mathrm{~K})$ & Data type & $N_{d}$ & $w_{d}^{\text {init }}$ & Reference \\
\hline acetone & $\mathrm{CH}_{\mathrm{n}}, \mathrm{CH}_{\mathrm{n}} \mathrm{CO}$ & $\left(\mathrm{CH}_{3}\right)\left(\mathrm{CH}_{3} \mathrm{CO}\right)$ & $309-340$ & VLE & 12 & 1.00 & Othmer and Benenati (1945) \\
\hline acetone & $\mathrm{CH}_{\mathrm{n}}, \mathrm{CH}_{\mathrm{n}} \mathrm{CO}$ & $\left(\mathrm{CH}_{3}\right)\left(\mathrm{CH}_{3} \mathrm{CO}\right)$ & $318-345$ & VLE & 13 & 1.00 & Othmer and Benenati (1945) \\
\hline acetone & $\mathrm{CH}_{\mathrm{n}}, \mathrm{CH}_{\mathrm{n}} \mathrm{CO}$ & $\left(\mathrm{CH}_{3}\right)\left(\mathrm{CH}_{3} \mathrm{CO}\right)$ & $331-363$ & VLE & 10 & 1.00 & Othmer and Benenati (1945) \\
\hline acetone & $\mathrm{CH}_{\mathrm{n}}, \mathrm{CH}_{\mathrm{n}} \mathrm{CO}$ & $\left(\mathrm{CH}_{3}\right)\left(\mathrm{CH}_{3} \mathrm{CO}\right)$ & $330-361$ & VLE & 13 & 1.00 & Othmer et al. (1952) \\
\hline acetone & $\mathrm{CH}_{\mathrm{n}}, \mathrm{CH}_{\mathrm{n}} \mathrm{CO}$ & $\left(\mathrm{CH}_{3}\right)\left(\mathrm{CH}_{3} \mathrm{CO}\right)$ & $371-396$ & VLE & 12 & 1.00 & Othmer et al. (1952) \\
\hline 2-butanone & $\mathrm{CH}_{\mathrm{n}}, \mathrm{CH}_{\mathrm{n}} \mathrm{CO}$ & $\left(\mathrm{CH}_{3}\right)\left(\mathrm{CH}_{2}\right)\left(\mathrm{CH}_{3} \mathrm{CO}\right)$ & $198-273$ & SLE & 19 & 5.00 & Lohmann et al. (1997) \\
\hline 2-butanone & $\mathrm{CH}_{\mathrm{n}}, \mathrm{CH}_{\mathrm{n}} \mathrm{CO}$ & $\left(\mathrm{CH}_{3}\right)\left(\mathrm{CH}_{2}\right)\left(\mathrm{CH}_{3} \mathrm{CO}\right)$ & 293 & VLE & 5 & 0.00 & Gmehling et al. (1988) \\
\hline 2-butanone & $\mathrm{CH}_{\mathrm{n}}, \mathrm{CH}_{\mathrm{n}} \mathrm{CO}$ & $\left(\mathrm{CH}_{3}\right)\left(\mathrm{CH}_{2}\right)\left(\mathrm{CH}_{3} \mathrm{CO}\right)$ & 308 & VLE & 4 & 1.00 & Gmehling et al. (1988) \\
\hline 2-butanone & $\mathrm{CH}_{\mathrm{n}}, \mathrm{CH}_{\mathrm{n}} \mathrm{CO}$ & $\left(\mathrm{CH}_{3}\right)\left(\mathrm{CH}_{2}\right)\left(\mathrm{CH}_{3} \mathrm{CO}\right)$ & 323 & VLE & 4 & 1.00 & Gmehling et al. (1988) \\
\hline 2-butanone & $\mathrm{CH}_{\mathrm{n}}, \mathrm{CH}_{\mathrm{n}} \mathrm{CO}$ & $\left(\mathrm{CH}_{3}\right)\left(\mathrm{CH}_{2}\right)\left(\mathrm{CH}_{3} \mathrm{CO}\right)$ & 323 & VLE & 15 & 1.00 & Gaube et al. (1996) \\
\hline 2-butanone & $\mathrm{CH}_{\mathrm{n}}, \mathrm{CH}_{\mathrm{n}} \mathrm{CO}$ & $\left(\mathrm{CH}_{3}\right)\left(\mathrm{CH}_{2}\right)\left(\mathrm{CH}_{3} \mathrm{CO}\right)$ & 333 & VLE & 20 & 1.00 & Zou and Prausnitz (1987) \\
\hline 2-butanone & $\mathrm{CH}_{\mathrm{n}}, \mathrm{CH}_{\mathrm{n}} \mathrm{CO}$ & $\left(\mathrm{CH}_{3}\right)\left(\mathrm{CH}_{2}\right)\left(\mathrm{CH}_{3} \mathrm{CO}\right)$ & 343 & VLE & 22 & 1.00 & Zou and Prausnitz (1987) \\
\hline 2-butanone & $\mathrm{CH}_{\mathrm{n}}, \mathrm{CH}_{\mathrm{n}} \mathrm{CO}$ & $\left(\mathrm{CH}_{3}\right)\left(\mathrm{CH}_{2}\right)\left(\mathrm{CH}_{3} \mathrm{CO}\right)$ & $313-326$ & VLE & 8 & 1.00 & Gmehling et al. (1981) \\
\hline 2-butanone & $\mathrm{CH}_{\mathrm{n}}, \mathrm{CH}_{\mathrm{n}} \mathrm{CO}$ & $\left(\mathrm{CH}_{3}\right)\left(\mathrm{CH}_{2}\right)\left(\mathrm{CH}_{3} \mathrm{CO}\right)$ & $330-338$ & VLE & 8 & 1.00 & Gmehling et al. (1981) \\
\hline 2-butanone & $\mathrm{CH}_{\mathrm{n}}, \mathrm{CH}_{\mathrm{n}} \mathrm{CO}$ & $\left(\mathrm{CH}_{3}\right)\left(\mathrm{CH}_{2}\right)\left(\mathrm{CH}_{3} \mathrm{CO}\right)$ & $340-348$ & VLE & 8 & 1.00 & Gmehling et al. (1981) \\
\hline 2-butanone & $\mathrm{CH}_{\mathrm{n}}, \mathrm{CH}_{\mathrm{n}} \mathrm{CO}$ & $\left(\mathrm{CH}_{3}\right)\left(\mathrm{CH}_{2}\right)\left(\mathrm{CH}_{3} \mathrm{CO}\right)$ & $347-363$ & VLE & 8 & 1.00 & Gmehling et al. (1981) \\
\hline 2-butanone & $\mathrm{CH}_{\mathrm{n}}, \mathrm{CH}_{\mathrm{n}} \mathrm{CO}$ & $\left(\mathrm{CH}_{3}\right)\left(\mathrm{CH}_{2}\right)\left(\mathrm{CH}_{3} \mathrm{CO}\right)$ & $385-406$ & VLE & 19 & 1.00 & Othmer et al. (1952) \\
\hline 2-pentanone & $\mathrm{CH}_{\mathrm{n}}, \mathrm{CH}_{\mathrm{n}} \mathrm{CO}$ & $\left(\mathrm{CH}_{3}\right)\left(\mathrm{CH}_{2}\right)_{2}\left(\mathrm{CH}_{3} \mathrm{CO}\right)$ & $273-363$ & solubil. & 20 & 1.00 & Stephenson (1992) \\
\hline 3-pentanone & $\mathrm{CH}_{\mathrm{n}}, \mathrm{CH}_{\mathrm{n}} \mathrm{CO}$ & $\left(\mathrm{CH}_{3}\right)_{2}\left(\mathrm{CH}_{2}\right)\left(\mathrm{CH}_{2} \mathrm{CO}\right)$ & $273-353$ & solubil. & 18 & 1.00 & Stephenson (1992) \\
\hline 3-pentanone & $\mathrm{CH}_{\mathrm{n}}, \mathrm{CH}_{\mathrm{n}} \mathrm{CO}$ & $\left(\mathrm{CH}_{3}\right)_{2}\left(\mathrm{CH}_{2}\right)\left(\mathrm{CH}_{2} \mathrm{CO}\right)$ & 323 & VLE & 12 & 1.00 & Gmehling and Onken (2003b) \\
\hline 3-pentanone & $\mathrm{CH}_{\mathrm{n}}, \mathrm{CH}_{\mathrm{n}} \mathrm{CO}$ & $\left(\mathrm{CH}_{3}\right)_{2}\left(\mathrm{CH}_{2}\right)\left(\mathrm{CH}_{2} \mathrm{CO}\right)$ & 338 & VLE & 12 & 0.50 & Gmehling and Onken (2003b) \\
\hline 3-pentanone & $\mathrm{CH}_{\mathrm{n}}, \mathrm{CH}_{\mathrm{n}} \mathrm{CO}$ & $\left(\mathrm{CH}_{3}\right)_{2}\left(\mathrm{CH}_{2}\right)\left(\mathrm{CH}_{2} \mathrm{CO}\right)$ & 353 & VLE & 12 & 0.50 & Gmehling and Onken (2003b) \\
\hline 3-pentanone & $\mathrm{CH}_{\mathrm{n}}, \mathrm{CH}_{\mathrm{n}} \mathrm{CO}$ & $\left(\mathrm{CH}_{3}\right)_{2}\left(\mathrm{CH}_{2}\right)\left(\mathrm{CH}_{2} \mathrm{CO}\right)$ & 368 & VLE & 12 & 0.50 & Gmehling and Onken (2003b) \\
\hline 3-pentanone & $\mathrm{CH}_{\mathrm{n}}, \mathrm{CH}_{\mathrm{n}} \mathrm{CO}$ & $\left(\mathrm{CH}_{3}\right)_{2}\left(\mathrm{CH}_{2}\right)\left(\mathrm{CH}_{2} \mathrm{CO}\right)$ & 383 & VLE & 12 & 1.00 & Gmehling and Onken (2003b) \\
\hline \multicolumn{8}{|l|}{ - water + ether systems - } \\
\hline diethyl ether & $\mathrm{CH}_{\mathrm{n}}, \mathrm{CH}_{\mathrm{n}} \mathrm{O}$ & $\left(\mathrm{CH}_{3}\right)_{2}\left(\mathrm{CH}_{2}\right)\left(\mathrm{CH}_{2} \mathrm{O}\right)$ & $269-272$ & SLE(org) ${ }^{\mathrm{d}}$ & 7 & 5.00 & Lalande (1934) \\
\hline diethyl ether & $\mathrm{CH}_{\mathrm{n}}, \mathrm{CH}_{\mathrm{n}} \mathrm{O}$ & $\left(\mathrm{CH}_{3}\right)_{2}\left(\mathrm{CH}_{2}\right)\left(\mathrm{CH}_{2} \mathrm{O}\right)$ & $269-303$ & solubli. & 14 & 0.20 & Hill (1923) \\
\hline diethyl ether & $\mathrm{CH}_{\mathrm{n}}, \mathrm{CH}_{\mathrm{n}} \mathrm{O}$ & $\left(\mathrm{CH}_{3}\right)_{2}\left(\mathrm{CH}_{2}\right)\left(\mathrm{CH}_{2} \mathrm{O}\right)$ & $307-367$ & VLE & 10 & 0.05 & Borisova et al. (1983) \\
\hline 2-methoxyethanol & $\mathrm{CH}_{\mathrm{n}}, \mathrm{CH}_{\mathrm{n}}^{[\mathrm{OH}]}, \mathrm{CH}_{\mathrm{n}} \mathrm{O}, \mathrm{OH}$ & $\left(\mathrm{CH}_{2}\right)\left(\mathrm{CH}_{2}^{[\mathrm{OH}]}\right)\left(\mathrm{CH}_{3} \mathrm{O}\right)(\mathrm{OH})$ & 343 & VLE & 16 & 0.50 & Chiavone-Filho et al. (1993) \\
\hline 2-methoxyethanol & $\mathrm{CH}_{\mathrm{n}}, \mathrm{CH}_{\mathrm{n}}^{[\mathrm{OH}]}, \mathrm{CH}_{\mathrm{n}} \mathrm{O}, \mathrm{OH}$ & $\left(\mathrm{CH}_{2}\right)\left(\mathrm{CH}_{2}^{[\mathrm{OH}]}\right)\left(\mathrm{CH}_{3} \mathrm{O}\right)(\mathrm{OH})$ & 363 & VLE & 16 & 0.50 & Chiavone-Filho et al. (1993) \\
\hline 2-methoxyethanol & $\mathrm{CH}_{\mathrm{n}}, \mathrm{CH}_{\mathrm{n}}^{[\mathrm{OH}]}, \mathrm{CH}_{\mathrm{n}} \mathrm{O}, \mathrm{OH}$ & $\left(\mathrm{CH}_{2}\right)\left(\mathrm{CH}_{2}^{[\mathrm{OH}]}\right)\left(\mathrm{CH}_{3} \mathrm{O}\right)(\mathrm{OH})$ & $373-394$ & VLE & 12 & 0.50 & Gmehling and Onken (2003a) \\
\hline 2-ethoxyethanol & $\mathrm{CH}_{\mathrm{n}}, \mathrm{CH}_{\mathrm{n}}^{[\mathrm{OH}]}, \mathrm{CH}_{\mathrm{n}} \mathrm{O}, \mathrm{OH}$ & $\left(\mathrm{CH}_{3}\right)\left(\mathrm{CH}_{2}\right)\left(\mathrm{CH}_{2}^{[\mathrm{OH}]}\right)\left(\mathrm{CH}_{2} \mathrm{O}\right)(\mathrm{OH})$ & 343 & VLE & 20 & 0.50 & Chiavone-Filho et al. (1993) \\
\hline 2-ethoxyethanol & $\mathrm{CH}_{\mathrm{n}}, \mathrm{CH}_{\mathrm{n}}^{[\mathrm{OH}]}, \mathrm{CH}_{\mathrm{n}} \mathrm{O}, \mathrm{OH}$ & $\left(\mathrm{CH}_{3}\right)\left(\mathrm{CH}_{2}\right)\left(\mathrm{CH}_{2}^{[\mathrm{OH}]}\right)\left(\mathrm{CH}_{2} \mathrm{O}\right)(\mathrm{OH})$ & 363 & VLE & 18 & 0.50 & Chiavone-Filho et al. (1993) \\
\hline 2-ethoxyethanol & $\mathrm{CH}_{\mathrm{n}}, \mathrm{CH}_{\mathrm{n}}^{[\mathrm{OH}]}, \mathrm{CH}_{\mathrm{n}} \mathrm{O}, \mathrm{OH}$ & $\left(\mathrm{CH}_{3}\right)\left(\mathrm{CH}_{2}\right)\left(\mathrm{CH}_{2}^{[\mathrm{OH}]}\right)\left(\mathrm{CH}_{2} \mathrm{O}\right)(\mathrm{OH})$ & $373-407$ & VLE & 34 & 0.50 & Hirata and Hoshino (1982) \\
\hline 2-ethoxyethanol & $\mathrm{CH}_{\mathrm{n}}, \mathrm{CH}_{\mathrm{n}}^{[\mathrm{OH}]}, \mathrm{CH}_{\mathrm{n}} \mathrm{O}, \mathrm{OH}$ & $\left(\mathrm{CH}_{3}\right)\left(\mathrm{CH}_{2}\right)\left(\mathrm{CH}_{2}^{[\mathrm{OH}]}\right)\left(\mathrm{CH}_{2} \mathrm{O}\right)(\mathrm{OH})$ & $372-406$ & VLE & 17 & 0.50 & Gmehling and Onken (2003b) \\
\hline 2-butoxyethanol & $\mathrm{CH}_{\mathrm{n}}, \mathrm{CH}_{\mathrm{n}}^{[\mathrm{OH}]}, \mathrm{CH}_{\mathrm{n}} \mathrm{O}, \mathrm{OH}$ & $\left(\mathrm{CH}_{3}\right)\left(\mathrm{CH}_{2}\right)_{3}\left(\mathrm{CH}_{2}^{[\mathrm{OH}]}\right)\left(\mathrm{CH}_{2} \mathrm{O}\right)(\mathrm{OH})$ & $252-273$ & SLE & 23 & 0.50 & Koga et al. (1994) \\
\hline 2-butoxyethanol & $\mathrm{CH}_{\mathrm{n}}, \mathrm{CH}_{\mathrm{n}}^{[\mathrm{OH}]}, \mathrm{CH}_{\mathrm{n}} \mathrm{O}, \mathrm{OH}$ & $\left(\mathrm{CH}_{3}\right)\left(\mathrm{CH}_{2}\right)_{3}\left(\mathrm{CH}_{2}^{[\mathrm{OH}]}\right)\left(\mathrm{CH}_{2} \mathrm{O}\right)(\mathrm{OH})$ & $261-273$ & SLE & 23 & 5.00 & Koga et al. (1994) \\
\hline 2-butoxyethanol & $\mathrm{CH}_{\mathrm{n}}, \mathrm{CH}_{\mathrm{n}}^{[\mathrm{OH}]}, \mathrm{CH}_{\mathrm{n}} \mathrm{O}, \mathrm{OH}$ & $\left(\mathrm{CH}_{3}\right)\left(\mathrm{CH}_{2}\right)_{3}\left(\mathrm{CH}_{2}^{[\mathrm{OH}]}\right)\left(\mathrm{CH}_{2} \mathrm{O}\right)(\mathrm{OH})$ & 298 & VLE & 8 & 0.00 & Scatchard and Wilson (1964) \\
\hline 2-butoxyethanol & $\mathrm{CH}_{\mathrm{n}}, \mathrm{CH}_{\mathrm{n}}^{[\mathrm{OH}]}, \mathrm{CH}_{\mathrm{n}} \mathrm{O}, \mathrm{OH}$ & $\left(\mathrm{CH}_{3}\right)\left(\mathrm{CH}_{2}\right)_{3}\left(\mathrm{CH}_{2}^{[\mathrm{OH}]}\right)\left(\mathrm{CH}_{2} \mathrm{O}\right)(\mathrm{OH})$ & 318 & VLE & 8 & 0.05 & Scatchard and Wilson (1964) \\
\hline 2-butoxyethanol & $\mathrm{CH}_{\mathrm{n}}, \mathrm{CH}_{\mathrm{n}}^{[\mathrm{OH}]}, \mathrm{CH}_{\mathrm{n}} \mathrm{O}, \mathrm{OH}$ & $\left(\mathrm{CH}_{3}\right)\left(\mathrm{CH}_{2}\right)_{3}\left(\mathrm{CH}_{2}^{[\mathrm{OH}]}\right)\left(\mathrm{CH}_{2} \mathrm{O}\right)(\mathrm{OH})$ & 338 & VLE & 7 & 0.05 & Scatchard and Wilson (1964) \\
\hline 2-butoxyethanol & $\mathrm{CH}_{\mathrm{n}}, \mathrm{CH}_{\mathrm{n}}^{[\mathrm{OH}]}, \mathrm{CH}_{\mathrm{n}} \mathrm{O}, \mathrm{OH}$ & $\left(\mathrm{CH}_{3}\right)\left(\mathrm{CH}_{2}\right)_{3}\left(\mathrm{CH}_{2}^{[\mathrm{OH}]}\right)\left(\mathrm{CH}_{2} \mathrm{O}\right)(\mathrm{OH})$ & 358 & VLE & 7 & 0.50 & Scatchard and Wilson (1964) \\
\hline 2-butoxyethanol & $\mathrm{CH}_{\mathrm{n}}, \mathrm{CH}_{\mathrm{n}}^{[\mathrm{OH}]}, \mathrm{CH}_{\mathrm{n}} \mathrm{O}, \mathrm{OH}$ & $\left(\mathrm{CH}_{3}\right)\left(\mathrm{CH}_{2}\right)_{3}\left(\mathrm{CH}_{2}^{[\mathrm{OH}]}\right)\left(\mathrm{CH}_{2} \mathrm{O}\right)(\mathrm{OH})$ & 358 & VLE & 19 & 0.50 & Chiavone-Filho et al. (1993) \\
\hline 2-butoxyethanol & $\mathrm{CH}_{\mathrm{n}}, \mathrm{CH}_{\mathrm{n}}^{[\mathrm{OH}]}, \mathrm{CH}_{\mathrm{n}} \mathrm{O}, \mathrm{OH}$ & $\left(\mathrm{CH}_{3}\right)\left(\mathrm{CH}_{2}\right)_{3}\left(\mathrm{CH}_{2}^{[\mathrm{OH}]}\right)\left(\mathrm{CH}_{2} \mathrm{O}\right)(\mathrm{OH})$ & 363 & VLE & 22 & 0.50 & Escobedo-Alvarado and Sandler (1999) \\
\hline 2-butoxyethanol & $\mathrm{CH}_{\mathrm{n}}, \mathrm{CH}_{\mathrm{n}}^{[\mathrm{OH}]}, \mathrm{CH}_{\mathrm{n}} \mathrm{O}, \mathrm{OH}$ & $\left(\mathrm{CH}_{3}\right)\left(\mathrm{CH}_{2}\right)_{3}\left(\mathrm{CH}_{2}^{[\mathrm{OH}]}\right)\left(\mathrm{CH}_{2} \mathrm{O}\right)(\mathrm{OH})$ & 368 & VLE & 19 & 0.50 & Chiavone-Filho et al. (1993) \\
\hline 2-butoxyethanol & $\mathrm{CH}_{\mathrm{n}}, \mathrm{CH}_{\mathrm{n}}^{[\mathrm{OH}]}, \mathrm{CH}_{\mathrm{n}} \mathrm{O}, \mathrm{OH}$ & $\left(\mathrm{CH}_{3}\right)\left(\mathrm{CH}_{2}\right)_{3}\left(\mathrm{CH}_{2}^{[\mathrm{OH}]}\right)\left(\mathrm{CH}_{2} \mathrm{O}\right)(\mathrm{OH})$ & 371 & VLE & 20 & 0.50 & Escobedo-Alvarado and Sandler (1999) \\
\hline 2-butoxyethanol & $\mathrm{CH}_{\mathrm{n}}, \mathrm{CH}_{\mathrm{n}}^{[\mathrm{OH}]}, \mathrm{CH}_{\mathrm{n}} \mathrm{O}, \mathrm{OH}$ & $\left(\mathrm{CH}_{3}\right)\left(\mathrm{CH}_{2}\right)_{3}\left(\mathrm{CH}_{2}^{[\mathrm{OH}]}\right)\left(\mathrm{CH}_{2} \mathrm{O}\right)(\mathrm{OH})$ & 383 & VLE & 21 & 0.50 & Schneider and Wilhelm (1959) \\
\hline 2-butoxyethanol & $\mathrm{CH}_{\mathrm{n}}, \mathrm{CH}_{\mathrm{n}}^{[\mathrm{OH}]}, \mathrm{CH}_{\mathrm{n}} \mathrm{O}, \mathrm{OH}$ & $\left(\mathrm{CH}_{3}\right)\left(\mathrm{CH}_{2}\right)_{3}\left(\mathrm{CH}_{2}^{[\mathrm{OH}]}\right)\left(\mathrm{CH}_{2} \mathrm{O}\right)(\mathrm{OH})$ & $372-423$ & VLE & 8 & 0.50 & Newman et al. (1949) \\
\hline 2-isopropoxyethanol & $\mathrm{CH}_{\mathrm{n}}, \mathrm{CH}_{\mathrm{n}}^{[\mathrm{OH}]}, \mathrm{CH}_{\mathrm{n}} \mathrm{O}, \mathrm{OH}$ & $\left(\mathrm{CH}_{3}\right)_{2}(\mathrm{CH})\left(\mathrm{CH}_{2}^{[\mathrm{OH}]}\right)\left(\mathrm{CH}_{2} \mathrm{O}\right)(\mathrm{OH})$ & 358 & SLE & 16 & 0.50 & Chiavone-Filho et al. (1993) \\
\hline 2-isopropoxyethanol & $\mathrm{CH}_{\mathrm{n}}, \mathrm{CH}_{\mathrm{n}}^{[\mathrm{OH}]}, \mathrm{CH}_{\mathrm{n}} \mathrm{O}, \mathrm{OH}$ & $\left(\mathrm{CH}_{3}\right)_{2}(\mathrm{CH})\left(\mathrm{CH}_{2}^{[\mathrm{OH}]}\right)\left(\mathrm{CH}_{2} \mathrm{O}\right)(\mathrm{OH})$ & 368 & SLE & 16 & 0.50 & Chiavone-Filho et al. (1993) \\
\hline 1-methoxy-2-propanol & $\mathrm{CH}_{\mathrm{n}}, \mathrm{CH}_{\mathrm{n}}^{[\mathrm{OH}]}, \mathrm{CH}_{\mathrm{n}} \mathrm{O}, \mathrm{OH}$ & $\left(\mathrm{CH}_{3}\right)\left(\mathrm{CH}_{2}\right)\left(\mathrm{CH}^{[\mathrm{OH}]}\right)\left(\mathrm{CH}_{3} \mathrm{O}\right)(\mathrm{OH})$ & 353 & VLE & 13 & 0.50 & Chiavone-Filho et al. (1993) \\
\hline 1-methoxy-2-propanol & $\mathrm{CH}_{\mathrm{n}}, \mathrm{CH}_{\mathrm{n}}^{[\mathrm{OH}]}, \mathrm{CH}_{\mathrm{n}} \mathrm{O}, \mathrm{OH}$ & $\left(\mathrm{CH}_{3}\right)\left(\mathrm{CH}_{2}\right)\left(\mathrm{CH}^{[\mathrm{OH}]}\right)\left(\mathrm{CH}_{3} \mathrm{O}\right)(\mathrm{OH})$ & 363 & VLE & 13 & 0.50 & Chiavone-Filho et al. (1993) \\
\hline \multicolumn{8}{|l|}{ - water + ester systems - } \\
\hline methyl acetate & $\mathrm{CH}_{\mathrm{n}}, \mathrm{CCOO}$ & $\left(\mathrm{CH}_{3}\right)\left(\mathrm{CH}_{3} \mathrm{COO}\right)$ & $233-273$ & SLE & 7 & 5.00 & Ahlers (1998) \\
\hline methyl acetate & $\mathrm{CH}_{\mathrm{n}}, \mathrm{CCOO}$ & $\left(\mathrm{CH}_{3}\right)\left(\mathrm{CH}_{3} \mathrm{COO}\right)$ & 298 & VLE & 5 & 0.00 & Gmehling and Onken (1977) \\
\hline methyl acetate & $\mathrm{CH}_{\mathrm{n}}, \mathrm{CCOO}$ & $\left(\mathrm{CH}_{3}\right)\left(\mathrm{CH}_{3} \mathrm{COO}\right)$ & 308 & VLE & 5 & 1.00 & Gmehling and Onken (1977) \\
\hline methyl acetate & $\mathrm{CH}_{\mathrm{n}}, \mathrm{CCOO}$ & $\left(\mathrm{CH}_{3}\right)\left(\mathrm{CH}_{3} \mathrm{COO}\right)$ & 323 & VLE & 8 & 0.02 & Gmehling and Onken (1977) \\
\hline methyl acetate & $\mathrm{CH}_{\mathrm{n}}, \mathrm{CCOO}$ & $\left(\mathrm{CH}_{3}\right)\left(\mathrm{CH}_{3} \mathrm{COO}\right)$ & 323 & VLE & 30 & 1.00 & Loehe et al. (1983) \\
\hline methyl acetate & $\mathrm{CH}_{\mathrm{n}}, \mathrm{CCOO}$ & $\left(\mathrm{CH}_{3}\right)\left(\mathrm{CH}_{3} \mathrm{COO}\right)$ & 333 & VLE & 8 & 1.00 & Gmehling and Onken (1977) \\
\hline methyl acetate & $\mathrm{CH}_{\mathrm{n}}, \mathrm{CCOO}$ & $\left(\mathrm{CH}_{3}\right)\left(\mathrm{CH}_{3} \mathrm{COO}\right)$ & 343 & VLE & 8 & 1.00 & Gmehling and Onken (1977) \\
\hline methyl acetate & $\mathrm{CH}_{\mathrm{n}}, \mathrm{CCOO}$ & $\left(\mathrm{CH}_{3}\right)\left(\mathrm{CH}_{3} \mathrm{COO}\right)$ & 353 & VLE & 8 & 1.00 & Gmehling and Onken (1977) \\
\hline methyl acetate & $\mathrm{CH}_{\mathrm{n}}, \mathrm{CCOO}$ & $\left(\mathrm{CH}_{3}\right)\left(\mathrm{CH}_{3} \mathrm{COO}\right)$ & $330-369$ & VLE & 12 & 1.00 & Álvarez et al. (2011) \\
\hline ethyl acetate & $\mathrm{CH}_{\mathrm{n}}, \mathrm{CCOO}$ & $\left(\mathrm{CH}_{3}\right)\left(\mathrm{CH}_{2}\right)\left(\mathrm{CH}_{3} \mathrm{COO}\right)$ & $273-344$ & solubil. & 16 & 1.00 & Stephenson and Stuart (1986) \\
\hline ethyl acetate & $\mathrm{CH}_{\mathrm{n}}, \mathrm{CCOO}$ & $\left(\mathrm{CH}_{3}\right)\left(\mathrm{CH}_{2}\right)\left(\mathrm{CH}_{3} \mathrm{COO}\right)$ & 323 & VLE & 9 & 1.00 & Gmehling et al. (1988) \\
\hline ethyl acetate & $\mathrm{CH}_{\mathrm{n}}, \mathrm{CCOO}$ & $\left(\mathrm{CH}_{3}\right)\left(\mathrm{CH}_{2}\right)\left(\mathrm{CH}_{3} \mathrm{COO}\right)$ & 333 & VLE & 8 & 1.00 & Gmehling et al. (1988) \\
\hline ethyl acetate & $\mathrm{CH}_{\mathrm{n}}, \mathrm{CCOO}$ & $\left(\mathrm{CH}_{3}\right)\left(\mathrm{CH}_{2}\right)\left(\mathrm{CH}_{3} \mathrm{COO}\right)$ & 343 & VLE & 9 & 1.00 & Gmehling et al. (1988) \\
\hline ethyl acetate & $\mathrm{CH}_{\mathrm{n}}, \mathrm{CCOO}$ & $\left(\mathrm{CH}_{3}\right)\left(\mathrm{CH}_{2}\right)\left(\mathrm{CH}_{3} \mathrm{COO}\right)$ & 353 & VLE & 9 & 1.00 & Gmehling et al. (1988) \\
\hline ethyl acetate & $\mathrm{CH}_{\mathrm{n}}, \mathrm{CCOO}$ & $\left(\mathrm{CH}_{3}\right)\left(\mathrm{CH}_{2}\right)\left(\mathrm{CH}_{3} \mathrm{COO}\right)$ & $345-367$ & VLE & 9 & 1.00 & Gmehling et al. (1988) \\
\hline
\end{tabular}


Table 1. Continued.

\begin{tabular}{|c|c|c|c|c|c|c|c|}
\hline Organic compounds & Org. main groups & Chemical formula (subgroups) & $T(\mathrm{~K})$ & Data type & $N_{d}$ & $w_{d}^{\text {init }}$ & Reference \\
\hline ethyl acetate & $\mathrm{CH}_{\mathrm{n}}, \mathrm{CCOO}$ & $\left(\mathrm{CH}_{3}\right)\left(\mathrm{CH}_{2}\right)\left(\mathrm{CH}_{3} \mathrm{COO}\right)$ & $344-349$ & VLE & 11 & 0.20 & Gmehling et al. (1988) \\
\hline 1-propyl acetate & $\mathrm{CH}_{\mathrm{n}}, \mathrm{CCOO}$ & $\left(\mathrm{CH}_{3}\right)\left(\mathrm{CH}_{2}\right)_{2}\left(\mathrm{CH}_{3} \mathrm{COO}\right)$ & $273-353$ & solubil. & 18 & 1.00 & Stephenson and Stuart (1986) \\
\hline 1-propyl acetate & $\mathrm{CH}_{\mathrm{n}}, \mathrm{CCOO}$ & $\left(\mathrm{CH}_{3}\right)\left(\mathrm{CH}_{2}\right)_{2}\left(\mathrm{CH}_{3} \mathrm{COO}\right)$ & $324-338$ & VLE & 7 & 1.00 & Gmehling et al. (1988) \\
\hline 1-propyl acetate & $\mathrm{CH}_{\mathrm{n}}, \mathrm{CCOO}$ & $\left(\mathrm{CH}_{3}\right)\left(\mathrm{CH}_{2}\right)_{2}\left(\mathrm{CH}_{3} \mathrm{COO}\right)$ & $340-354$ & VLE & 7 & 1.00 & Gmehling et al. (1988) \\
\hline 1-propyl acetate & $\mathrm{CH}_{\mathrm{n}}, \mathrm{CCOO}$ & $\left(\mathrm{CH}_{3}\right)\left(\mathrm{CH}_{2}\right)_{2}\left(\mathrm{CH}_{3} \mathrm{COO}\right)$ & $350-365$ & VLE & 7 & 1.00 & Gmehling et al. (1988) \\
\hline 1-propyl acetate & $\mathrm{CH}_{\mathrm{n}}, \mathrm{CCOO}$ & $\left(\mathrm{CH}_{3}\right)\left(\mathrm{CH}_{2}\right)_{2}\left(\mathrm{CH}_{3} \mathrm{COO}\right)$ & $356-371$ & VLE & 7 & 1.00 & Gmehling et al. (1988) \\
\hline 1-propyl acetate & $\mathrm{CH}_{\mathrm{n}}, \mathrm{CCOO}$ & $\left(\mathrm{CH}_{3}\right)\left(\mathrm{CH}_{2}\right)_{2}\left(\mathrm{CH}_{3} \mathrm{COO}\right)$ & 338 & VLE & 7 & 1.00 & Gmehling et al. (1988) \\
\hline 1-propyl acetate & $\mathrm{CH}_{\mathrm{n}}, \mathrm{CCOO}$ & $\left(\mathrm{CH}_{3}\right)\left(\mathrm{CH}_{2}\right)_{2}\left(\mathrm{CH}_{3} \mathrm{COO}\right)$ & 353 & VLE & 7 & 1.00 & Gmehling et al. (1988) \\
\hline 1-butyl acetate & $\mathrm{CH}_{\mathrm{n}}, \mathrm{CCOO}$ & $\left(\mathrm{CH}_{3}\right)\left(\mathrm{CH}_{2}\right)_{3}\left(\mathrm{CH}_{3} \mathrm{COO}\right)$ & $273-364$ & solubil. & 20 & 0.50 & Stephenson and Stuart (1986) \\
\hline 1-butyl acetate & $\mathrm{CH}_{\mathrm{n}}, \mathrm{CCOO}$ & $\left(\mathrm{CH}_{3}\right)\left(\mathrm{CH}_{2}\right)_{3}\left(\mathrm{CH}_{3} \mathrm{COO}\right)$ & $364-397$ & VLE & 31 & 0.20 & Cho et al. (1983) \\
\hline isobutyl acetate & $\mathrm{CH}_{\mathrm{n}}, \mathrm{CCOO}$ & $\left(\mathrm{CH}_{3}\right)_{2}\left(\mathrm{CH}_{2}\right)(\mathrm{CH})\left(\mathrm{CH}_{3} \mathrm{COO}\right)$ & $273-353$ & solubil. & 18 & 0.50 & Stephenson and Stuart (1986) \\
\hline 2-butyl acetate & $\mathrm{CH}_{\mathrm{n}}, \mathrm{CCOO}$ & $\left(\mathrm{CH}_{3}\right)_{2}\left(\mathrm{CH}_{2}\right)(\mathrm{CH})\left(\mathrm{CH}_{3} \mathrm{COO}\right)$ & $273-364$ & solubil. & 20 & 0.50 & Stephenson and Stuart (1986) \\
\hline 1-pentyl acetate & $\mathrm{CH}_{\mathrm{n}}, \mathrm{CCOO}$ & $\left(\mathrm{CH}_{3}\right)\left(\mathrm{CH}_{2}\right)_{4}\left(\mathrm{CH}_{3} \mathrm{COO}\right)$ & $273-353$ & solubil. & 16 & 0.20 & Stephenson and Stuart (1986) \\
\hline 1-hexyl acetate & $\mathrm{CH}_{\mathrm{n}}, \mathrm{CCOO}$ & $\left(\mathrm{CH}_{3}\right)\left(\mathrm{CH}_{2}\right)_{5}\left(\mathrm{CH}_{3} \mathrm{COO}\right)$ & $273-363$ & solubil. & 20 & 0.20 & Stephenson and Stuart (1986) \\
\hline 1-hexyl acetate & $\mathrm{CH}_{\mathrm{n}}, \mathrm{CCOO}$ & $\left(\mathrm{CH}_{3}\right)\left(\mathrm{CH}_{2}\right)_{5}\left(\mathrm{CH}_{3} \mathrm{COO}\right)$ & $371-349$ & VLE & 6 & 0.001 & Bomshtein et al. (1983) \\
\hline \multicolumn{8}{|c|}{ - water + multifunctional aromatic compounds systems - } \\
\hline benzene & $\mathrm{ACH}_{\mathrm{n}}$ & $(\mathrm{ACH})_{6}$ & $293-353$ & solubil. & 8 & 1.00 & Udovenko (1963) \\
\hline benzene & $\mathrm{ACH}_{\mathrm{n}}$ & $(\mathrm{ACH})_{6}$ & $274-339$ & solubil. & 10 & 1.00 & Alexander (1959) \\
\hline benzene & $\mathrm{ACH}_{\mathrm{n}}$ & $(\mathrm{ACH})_{6}$ & $273-229$ & solubil. & 8 & 1.00 & May et al. (1983) \\
\hline benzene & $\mathrm{ACH}_{\mathrm{n}}$ & $(\mathrm{ACH})_{6}$ & $342-371$ & VLE & 20 & 0.01 & Gmehling and Onken (2003b) \\
\hline phenol & $\mathrm{ACH}_{\mathrm{n}}, \mathrm{ACOH}$ & $(\mathrm{ACH})_{5}(\mathrm{ACOH})$ & $284-314$ & SLE(org) $)^{d}$ & 23 & 0.20 & Paterno and Ampola (1897) \\
\hline phenol & $\mathrm{ACH}_{\mathrm{n}}, \mathrm{ACOH}$ & $(\mathrm{ACH})_{5}(\mathrm{ACOH})$ & $293-308$ & SLE (org) ${ }^{d}$ & 16 & 0.00 & Jaoui et al. (2002) \\
\hline phenol & $\mathrm{ACH}_{\mathrm{n}}, \mathrm{ACOH}$ & $(\mathrm{ACH})_{5}(\mathrm{ACOH})$ & 318 & VLE & 22 & 1.00 & Gmehling et al. (1981) \\
\hline phenol & $\mathrm{ACH}_{\mathrm{n}}, \mathrm{ACOH}$ & $(\mathrm{ACH})_{5}(\mathrm{ACOH})$ & $340-400$ & VLE & 21 & 1.0 & Kliment et al. (1964) \\
\hline phenol & $\mathrm{ACH}_{\mathrm{n}}, \mathrm{ACOH}$ & $(\mathrm{ACH})_{5}(\mathrm{ACOH})$ & $373-442$ & VLE & 15 & 1.0 & Schreinemakers $(1900)$ \\
\hline phenol & $\mathrm{ACH}_{\mathrm{n}}, \mathrm{ACOH}$ & $(\mathrm{ACH})_{5}(\mathrm{ACOH})$ & $373-455$ & VLE & 14 & 1.0 & Gmehling and Onken (2003b) \\
\hline phenol & $\mathrm{ACH}_{\mathrm{n}}, \mathrm{ACOH}$ & $(\mathrm{ACH})_{5}(\mathrm{ACOH})$ & $373-444$ & VLE & 11 & 1.00 & Tochigi et al. (1997) \\
\hline tert-butyl acetate & $\mathrm{CH}_{\mathrm{n}}, \mathrm{CCOO}$ & $\left(\mathrm{CH}_{3}\right)_{3}(\mathrm{C})\left(\mathrm{CH}_{3} \mathrm{COO}\right)$ & $273-354$ & solubil. & 18 & 0.50 & Stephenson and Stuart (1986) \\
\hline \multicolumn{8}{|c|}{ - water + aldehyde systems - } \\
\hline acetaldehyde & $\mathrm{CH}_{\mathrm{n}}, \mathrm{CHO}$ & $\left(\mathrm{CH}_{3}\right)(\mathrm{CHO})$ & 283 & VLE & 5 & 1.00 & d'Avila and Silva (1970) \\
\hline acetaldehyde & $\mathrm{CH}_{\mathrm{n}}, \mathrm{CHO}$ & $\left(\mathrm{CH}_{3}\right)(\mathrm{CHO})$ & 288 & VLE & 5 & 0.00 & d'Avila and Silva (1970) \\
\hline acetaldehyde & $\mathrm{CH}_{\mathrm{n}}, \mathrm{CHO}$ & $\left(\mathrm{CH}_{3}\right)(\mathrm{CHO})$ & 293 & VLE & 5 & 0.00 & d'Avila and Silva (1970) \\
\hline acetaldehyde & $\mathrm{CH}_{\mathrm{n}}, \mathrm{CHO}$ & $\left(\mathrm{CH}_{3}\right)(\mathrm{CHO})$ & 298 & VLE & 5 & 0.00 & d'Avila and Silva (1970) \\
\hline acetaldehyde & $\mathrm{CH}_{\mathrm{n}}, \mathrm{CHO}$ & $\left(\mathrm{CH}_{3}\right)(\mathrm{CHO})$ & 303 & VLE & 5 & 0.00 & d'Avila and Silva (1970) \\
\hline acetaldehyde & $\mathrm{CH}_{\mathrm{n}}, \mathrm{CHO}$ & $\left(\mathrm{CH}_{3}\right)(\mathrm{CHO})$ & $306-367$ & VLE & 5 & 1.00 & Coles and Popper (1950) \\
\hline propionaldehyde & $\mathrm{CH}_{\mathrm{n}}, \mathrm{CHO}$ & $\left(\mathrm{CH}_{3}\right)\left(\mathrm{CH}_{2}\right)(\mathrm{CHO})$ & $288-313$ & solubil. & 12 & 1.00 & Ferino et al. (1983) \\
\hline propionaldehyde & $\mathrm{CH}_{\mathrm{n}}, \mathrm{CHO}$ & $\left(\mathrm{CH}_{3}\right)\left(\mathrm{CH}_{2}\right)(\mathrm{CHO})$ & $321-342$ & VLE & 6 & 1.00 & Mozzhukhin et al. (1967) \\
\hline butyraldehyde & $\mathrm{CH}_{\mathrm{n}}, \mathrm{CHO}$ & $\left(\mathrm{CH}_{3}\right)\left(\mathrm{CH}_{2}\right)_{2}(\mathrm{CHO})$ & $278-313$ & solubil. & 16 & 1.00 & Ferino et al. (1983) \\
\hline butyraldehyde & $\mathrm{CH}_{\mathrm{n}}, \mathrm{CHO}$ & $\left(\mathrm{CH}_{3}\right)\left(\mathrm{CH}_{2}\right)_{2}(\mathrm{CHO})$ & 323 & VLE & 13 & 0.20 & Tapper et al. (1985) \\
\hline butyraldehyde & $\mathrm{CH}_{\mathrm{n}}, \mathrm{CHO}$ & $\left(\mathrm{CH}_{3}\right)\left(\mathrm{CH}_{2}\right)_{2}(\mathrm{CHO})$ & 338 & VLE & 12 & 0.20 & Tapper et al. (1985) \\
\hline \multicolumn{8}{|c|}{ - water + multifunctional systems - } \\
\hline glucose & $\mathrm{CH}_{\mathrm{n}}^{[\mathrm{OH}]}, \mathrm{OH}, \mathrm{CHO}[$ ether] & $\left(\mathrm{CH}_{2}^{[\mathrm{OH}]}\right)\left(\mathrm{CH}^{[\mathrm{OH}]}\right)_{4}(\mathrm{CHO}$ [ether] $)(\mathrm{OH})_{5}$ & $217-233$ & $a_{\mathrm{W}}\left(T_{\mathrm{hom}}\right)^{\mathrm{a}}$ & 9 & 1.00 & Miyata and Kanno (2005) \\
\hline glucose & $\mathrm{CH}_{\mathrm{n}}^{[\mathrm{OH}]}, \mathrm{OH}, \mathrm{CHO}[\mathrm{ether}]$ & $\left(\mathrm{CH}_{2}^{[\mathrm{OH}]}\right)\left(\mathrm{CH}^{[\mathrm{OH}]}\right)_{4}(\mathrm{CHO}[$ ether $])(\mathrm{OH})_{5}$ & $204-231$ & $a_{\mathrm{W}}\left(T_{\mathrm{hom}}\right)^{\mathrm{a}}$ & 5 & 1.00 & Zobrist et al. (2008) \\
\hline glucose & $\mathrm{CH}_{\mathrm{n}}^{[\mathrm{OH}]}, \mathrm{OH}, \mathrm{CHO}[$ ether] & $\left(\mathrm{CH}_{2}^{[\mathrm{OH}]}\right)\left(\mathrm{CH}^{[\mathrm{OH}]}\right)_{4}\left(\mathrm{CHO}[\right.$ ether $](\mathrm{OH})_{5}$ & $243-273$ & SLE & 8 & 5.00 & Young (1957) \\
\hline glucose & $\mathrm{CH}_{\mathrm{n}}^{[\mathrm{OH}]}, \mathrm{OH}, \mathrm{CHO}[$ ether] & $\left(\mathrm{CH}_{2}^{[\mathrm{OH}]}\right)\left(\mathrm{CH}^{[\mathrm{OH}]}\right)_{4}(\mathrm{CHO}[$ ether $])(\mathrm{OH})_{5}$ & $260-273$ & SLE & 5 & 5.00 & Zobrist et al. (2008) \\
\hline glucose & $\mathrm{CH}_{\mathrm{n}}^{[\mathrm{OH}]}, \mathrm{OH}, \mathrm{CHO}[$ ether] & $\left(\mathrm{CH}_{2}^{[\mathrm{OH}]}\right)\left(\mathrm{CH}^{[\mathrm{OH}]}\right)_{4}(\mathrm{CHO}[$ ether $])(\mathrm{OH})_{5}$ & 298 & $a_{\mathrm{W}}$ (bulk) & 20 & 0.00 & Ruegg and Blanc (1981) \\
\hline glucose & $\mathrm{CH}_{\mathrm{n}}^{[\mathrm{OH}]}, \mathrm{OH}, \mathrm{CHO}[$ ether] & $\left(\mathrm{CH}_{2}^{[\mathrm{OH}]}\right)\left(\mathrm{CH}^{[\mathrm{OH}]}\right)_{4}\left(\mathrm{CHO}[\right.$ ether] $)(\mathrm{OH})_{5}$ & 298 & $a_{\mathrm{W}}($ bulk) & 26 & 0.00 & Bonner and Breazeale (1965) \\
\hline glucose & $\mathrm{CH}_{\mathrm{n}}^{[\mathrm{OH}]}, \mathrm{OH}, \mathrm{CHO}[$ ether] & $\left(\mathrm{CH}_{2}^{[\mathrm{OH}]}\right)\left(\mathrm{CH}^{[\mathrm{OH}]}\right)_{4}(\mathrm{CHO}[$ ether $])(\mathrm{OH})_{5}$ & 298 & $a_{\mathrm{W}}($ bulk) & 8 & 0.00 & Peng et al. (2001) \\
\hline sucrose & $\mathrm{CH}_{\mathrm{n}}^{[\mathrm{OH}]}, \mathrm{OH}, \mathrm{CHO}[$ ether] & $(\mathrm{C})\left(\mathrm{CH}_{2}^{[\mathrm{OH}]}\right)_{3}\left(\mathrm{CH}^{[\mathrm{OH}]}\right)_{5}\left(\mathrm{CHO}[\text { ether] })_{3}(\mathrm{OH})_{8}\right.$ & $211-235$ & $a_{\mathrm{W}}\left(T_{\mathrm{hom}}\right)^{\mathrm{a}}$ & 16 & 1.00 & Kanno et al. (2007) \\
\hline sucrose & $\mathrm{CH}_{\mathrm{n}}^{[\mathrm{OH}]}, \mathrm{OH}, \mathrm{CHO}[\mathrm{ether}]$ & $(\mathrm{C})\left(\mathrm{CH}_{2}^{[\mathrm{OH}]}\right)_{3}\left(\mathrm{CH}^{[\mathrm{OH}]}\right)_{5}(\mathrm{CHO}[\text { ether }])_{3}(\mathrm{OH})_{8}$ & $217-232$ & $a_{\mathrm{w}}\left(T_{\mathrm{hom}}\right)^{\mathrm{a}}$ & 6 & 1.00 & Ganbavale et al. (2014) \\
\hline sucrose & $\mathrm{CH}_{\mathrm{n}}^{[\mathrm{OH}]}, \mathrm{OH}, \mathrm{CHO}[$ ether] & $(\mathrm{C})\left(\mathrm{CH}_{2}^{[\mathrm{OH}]}\right)_{3}\left(\mathrm{CH}^{[\mathrm{OH}]}\right)_{5}(\mathrm{CHO}[\mathrm{ether}])_{3}(\mathrm{OH})_{8}$ & $237-273$ & SLE & 10 & 5.00 & Ablett et al. (1992) \\
\hline sucrose & $\mathrm{CH}_{\mathrm{n}}^{[\mathrm{OH}]}, \mathrm{OH}, \mathrm{CHO}[$ ether] & (C) $\left(\mathrm{CH}_{2}^{[\mathrm{OH}]}\right)_{3}\left(\mathrm{CH}^{[\mathrm{OH}]}\right)_{5}(\mathrm{CHO}[\text { ether }])_{3}(\mathrm{OH})_{8}$ & $247-273$ & SLE & 9 & 5.00 & Williams and Carnahan (1990) \\
\hline sucrose & $\mathrm{CH}_{\mathrm{n}}^{[\mathrm{OH}]}, \mathrm{OH}, \mathrm{CHO}[$ ether] & $(\mathrm{C})\left(\mathrm{CH}_{2}^{[\mathrm{OH}]}\right)_{3}\left(\mathrm{CH}^{[\mathrm{OH}]}\right)_{5}\left(\mathrm{CHO}[\text { ether] })_{3}(\mathrm{OH})_{8}\right.$ & $259-271$ & SLE & 9 & 5.00 & Blond et al. (1997) \\
\hline sucrose & $\mathrm{CH}_{\mathrm{n}}^{[\mathrm{OH}]}, \mathrm{OH}, \mathrm{CHO}[$ ether] & $(\mathrm{C})\left(\mathrm{CH}_{2}^{[\mathrm{OH}]}\right)_{3}\left(\mathrm{CH}^{[\mathrm{OH}]}\right)_{5}\left(\mathrm{CHO}[\text { ether] })_{3}(\mathrm{OH})_{8}\right.$ & $261-272$ & SLE & 8 & 5.00 & Zobrist et al. (2008) \\
\hline sucrose & $\mathrm{CH}_{\mathrm{n}}^{[\mathrm{OH}]}, \mathrm{OH}, \mathrm{CHO}[$ ether] & (C) $\left(\mathrm{CH}_{2}^{[\mathrm{OH}]}\right)_{3}\left(\mathrm{CH}^{[\mathrm{OH}]}\right)_{5}\left(\mathrm{CHO}[\text { ether] })_{3}(\mathrm{OH})_{8}\right.$ & $262-273$ & SLE & 16 & 5.00 & Kanno et al. (2007) \\
\hline sucrose & $\mathrm{CH}_{\mathrm{n}}^{[\mathrm{OH}]}, \mathrm{OH}, \mathrm{CHO}[$ ether] & $(\mathrm{C})\left(\mathrm{CH}_{2}^{[\mathrm{OH}]}\right)_{3}\left(\mathrm{CH}^{[\mathrm{OH}]}\right)_{5}(\mathrm{CHO}[\text { ether }])_{3}(\mathrm{OH})_{8}$ & $264-272$ & SLE & 5 & 5.00 & Sei and Gonda (2006) \\
\hline sucrose & $\mathrm{CH}_{\mathrm{n}}^{[\mathrm{OH}]}, \mathrm{OH}, \mathrm{CHO}[$ ether] & (C) $\left(\mathrm{CH}_{2}^{[\mathrm{OH}]}\right)_{3}\left(\mathrm{CH}^{[\mathrm{OH}]}\right)_{5}(\mathrm{CHO}[\mathrm{ether}])_{3}(\mathrm{OH})_{8}$ & $269-273$ & SLE & 6 & 5.00 & Lerici et al. (2006) \\
\hline sucrose & $\mathrm{CH}_{\mathrm{n}}^{[\mathrm{OH}]}, \mathrm{OH}, \mathrm{CHO}[$ ether] & $(\mathrm{C})\left(\mathrm{CH}_{2}^{[\mathrm{OH}]}\right)_{3}\left(\mathrm{CH}^{[\mathrm{OH}]}\right)_{5}(\mathrm{CHO}[\text { ether }])_{3}(\mathrm{OH})_{8}$ & 289 & $a_{\mathrm{W}}($ bulk) & 8 & 0.00 & this work \\
\hline sucrose & $\mathrm{CH}_{\mathrm{n}}^{[\mathrm{OH}]}, \mathrm{OH}, \mathrm{CHO}[$ ether] & $(\mathrm{C})\left(\mathrm{CH}_{2}^{[\mathrm{OH}]}\right)_{3}\left(\mathrm{CH}^{[\mathrm{OH}]}\right)_{5}(\mathrm{CHO}[\text { ether }])_{3}(\mathrm{OH})_{8}$ & 298 & $a_{\mathrm{W}}($ bulk) & 8 & 0.00 & this work \\
\hline sucrose & $\mathrm{CH}_{\mathrm{n}}^{[\mathrm{OH}]}, \mathrm{OH}, \mathrm{CHO}[\mathrm{ether}]$ & $(\mathrm{C})\left(\mathrm{CH}_{2}^{[\mathrm{OH}]}\right)_{3}\left(\mathrm{CH}^{[\mathrm{OH}]}\right)_{5}(\mathrm{CHO}[\text { ether }])_{3}(\mathrm{OH})_{8}$ & 313 & $a_{\mathrm{W}}($ bulk $)$ & 8 & 1.00 & this work \\
\hline raffinose & $\mathrm{CH}_{\mathrm{n}}, \mathrm{CH}_{\mathrm{n}}^{[\mathrm{OH}]}, \mathrm{OH}, \mathrm{CHO}[\mathrm{ether}]$ & $\begin{array}{l}(\mathrm{C})(\mathrm{CH})\left(\mathrm{CH}_{2}^{[\mathrm{OH}]}\right)_{3}\left(\mathrm{CH}^{[\mathrm{OH}]}\right)_{8} \\
\left(\mathrm{CH}_{2} \mathrm{O}\right)\left(\mathrm{CHO}_{\text {ether }]}\right)_{4}(\mathrm{OH})_{11}\end{array}$ & $214-233$ & $a_{\mathrm{W}}\left(T_{\mathrm{hom}}\right)^{\mathrm{a}}$ & 4 & 1.00 & Zobrist et al. (2008) \\
\hline raffinose & $\mathrm{CH}_{\mathrm{n}}, \mathrm{CH}_{\mathrm{n}}^{[\mathrm{OH}]}, \mathrm{OH}, \mathrm{CHO}[\mathrm{ether}]$ & $\begin{array}{l}(\mathrm{C})(\mathrm{CH})\left(\mathrm{CH}_{2}^{[\mathrm{OH}]}\right)_{3}\left(\mathrm{CH}^{[\mathrm{OH}]}\right)_{8} \\
\left(\mathrm{CH}_{2} \mathrm{O}\right)\left(\mathrm{CHO}[\text { ether] })_{4}(\mathrm{OH})_{11}\right.\end{array}$ & $266-273$ & SLE & 4 & 5.00 & Zobrist et al. (2008) \\
\hline raffinose & $\mathrm{CH}_{\mathrm{n}}, \mathrm{CH}_{\mathrm{n}}^{[\mathrm{OH}]}, \mathrm{OH}, \mathrm{CHO}[\mathrm{ether}]$ & $\begin{array}{l}(\mathrm{C})(\mathrm{CH})\left(\mathrm{CH}_{2}^{[\mathrm{OH}]}\right)_{3}\left(\mathrm{CH}^{[\mathrm{OH}]}\right)_{8} \\
\left(\mathrm{CH}_{2} \mathrm{O}\right)\left(\mathrm{CHO}[\text { ether] })_{4}(\mathrm{OH})_{11}\right.\end{array}$ & 289 & $a_{\mathrm{W}}$ (bulk) & 4 & 0.00 & this work \\
\hline raffinose & $\mathrm{CH}_{\mathrm{n}}, \mathrm{CH}_{\mathrm{n}}^{[\mathrm{OH}]}, \mathrm{OH}, \mathrm{CHO}[\mathrm{ether}]$ & $\begin{array}{l}(\mathrm{C})(\mathrm{CH})\left(\mathrm{CH}_{2}^{[\mathrm{OH}]}\right)_{3}\left(\mathrm{CH}^{[\mathrm{OH}]}\right)_{8} \\
\left(\mathrm{CH}_{2} \mathrm{O}\right)(\mathrm{CHO}[\mathrm{ether}])_{4}(\mathrm{OH})_{11}\end{array}$ & 298 & $a_{\mathrm{W}}$ (bulk) & 5 & 0.00 & this work \\
\hline raffinose & $\mathrm{CH}_{\mathrm{n}}, \mathrm{CH}_{\mathrm{n}}^{[\mathrm{OH}]}, \mathrm{OH}, \mathrm{CHO}$ [ether] & $\begin{array}{l}(\mathrm{C})(\mathrm{CH})\left(\mathrm{CH}_{2}^{[\mathrm{OH}]}\right)_{3}\left(\mathrm{CH}^{[\mathrm{OH}]}\right)_{8} \\
\left(\mathrm{CH}_{2} \mathrm{O}\right)\left(\mathrm{CHO}[\text { ether })_{4}(\mathrm{OH})_{11}\right.\end{array}$ & 313 & $a_{\mathrm{W}}($ bulk) & 4 & 1.00 & this work \\
\hline levoglucosan & $\mathrm{CH}_{\mathrm{n}}, \mathrm{CH}_{\mathrm{n}}^{[\mathrm{OH}]}, \mathrm{OH}, \mathrm{CHO}[\mathrm{ether}]$ & $(\mathrm{CH})\left(\mathrm{CH}^{[\mathrm{OH}]}\right)_{3}\left(\mathrm{CH}_{2} \mathrm{O}\right)\left(\mathrm{CHO}[\right.$ ether] $)(\mathrm{OH})_{3}$ & $192-233$ & $a_{\mathrm{W}}\left(T_{\mathrm{hom}}\right)^{\mathrm{a}}$ & 8 & 1.00 & Zobrist et al. (2008) \\
\hline
\end{tabular}


Table 1. Continued.

\begin{tabular}{|c|c|c|c|c|c|c|c|}
\hline Organic compounds & Org. main groups & Chemical formula (subgroups) & $T(\mathrm{~K})$ & Data type & $N_{d}$ & $w_{d}^{\text {init }}$ & Reference \\
\hline levoglucosan & $\mathrm{CH}_{\mathrm{n}}, \mathrm{CH}_{\mathrm{n}}^{[\mathrm{OH}]}, \mathrm{OH}, \mathrm{CHO}[$ ether] & $(\mathrm{CH})\left(\mathrm{CH}^{[\mathrm{OH}]}\right)_{3}\left(\mathrm{CH}_{2} \mathrm{O}\right)\left(\mathrm{CHO}[\right.$ ether] $)(\mathrm{OH})_{3}$ & $187-230$ & $a_{\mathrm{w}}\left(T_{\mathrm{hom}}\right)^{\mathrm{a}}$ & 6 & 1.00 & Lienhard et al. (2012) \\
\hline levoglucosan & $\mathrm{CH}_{\mathrm{n}}, \mathrm{CH}_{\mathrm{n}}^{[\mathrm{OH}]}, \mathrm{OH}, \mathrm{CHO}[$ ether] & $(\mathrm{CH})\left(\mathrm{CH}^{[\mathrm{OH}]}\right)_{3}\left(\mathrm{CH}_{2} \mathrm{O}\right)(\mathrm{CHO}$ ether] $)(\mathrm{OH})_{3}$ & $255-272$ & SLE & 7 & 5.00 & Zobrist et al. (2008) \\
\hline levoglucosan & $\mathrm{CH}_{\mathrm{n}}, \mathrm{CH}_{\mathrm{n}}^{[\mathrm{OH}]}, \mathrm{OH}, \mathrm{CHO}[$ ether] & $(\mathrm{CH})\left(\mathrm{CH}^{[\mathrm{OH}]}\right)_{3}\left(\mathrm{CH}_{2} \mathrm{O}\right)\left(\mathrm{CHO}[\right.$ ether] $)(\mathrm{OH})_{3}$ & $254-272$ & SLE & 7 & 5.00 & Lienhard et al. (2012) \\
\hline levoglucosan & $\mathrm{CH}_{\mathrm{n}}, \mathrm{CH}_{\mathrm{n}}^{[\mathrm{OH}]}, \mathrm{OH}, \mathrm{CHO}[$ ether] & $(\mathrm{CH})\left(\mathrm{CH}^{[\mathrm{OH}]}\right)_{3}\left(\mathrm{CH}_{2} \mathrm{O}\right)\left(\mathrm{CHO}[\right.$ ether] $)(\mathrm{OH})_{3}$ & 291 & $a_{\mathrm{W}}$ (bulk) & 8 & 0.00 & Lienhard et al. (2012) \\
\hline levoglucosan & $\mathrm{CH}_{\mathrm{n}}, \mathrm{CH}_{\mathrm{n}}^{[\mathrm{OH}]}, \mathrm{OH}, \mathrm{CHO}[$ ether] & $(\mathrm{CH})\left(\mathrm{CH}^{[\mathrm{OH}]}\right)_{3}\left(\mathrm{CH}_{2} \mathrm{O}\right)(\mathrm{CHO}$ ether] $)(\mathrm{OH})_{3}$ & 296 & $a_{\mathrm{W}}$ (bulk) & 6 & 0.00 & Chan et al. (2005) \\
\hline levoglucosan & $\mathrm{CH}_{\mathrm{n}}, \mathrm{CH}_{\mathrm{n}}^{[\mathrm{OH}]}, \mathrm{OH}, \mathrm{CHO}[$ ether] & $(\mathrm{CH})\left(\mathrm{CH}^{[\mathrm{OH}]}\right)_{3}\left(\mathrm{CH}_{2} \mathrm{O}\right)(\mathrm{CHO}[\mathrm{ether}])(\mathrm{OH})_{3}$ & 298 & $a_{\mathrm{w}}$ (bulk) & 7 & 0.00 & Lienhard et al. (2012) \\
\hline glycolic acid & $\mathrm{CH}_{\mathrm{n}}^{[\mathrm{OH}]}, \mathrm{OH}, \mathrm{COOH}$ & $\left(\mathrm{CH}_{2}^{[\mathrm{OH}]}\right)(\mathrm{OH})(\mathrm{COOH})$ & $206-230$ & $a_{\mathrm{w}}\left(T_{\mathrm{hom}}\right)^{\mathrm{a}}$ & 4 & 1.00 & Ganbavale et al. (2014) \\
\hline glycolic acid & $\mathrm{CH}_{\mathrm{n}}^{[\mathrm{OH}]}, \mathrm{OH}, \mathrm{COOH}$ & $\left(\mathrm{CH}_{2}^{[\mathrm{OH}]}\right)(\mathrm{OH})(\mathrm{COOH})$ & $259-271$ & SLE & 4 & 5.00 & Ganbavale et al. (2014) \\
\hline glycolic acid & $\mathrm{CH}_{\mathrm{n}}^{[\mathrm{OH}]}, \mathrm{OH}, \mathrm{COOH}$ & $\left(\mathrm{CH}_{2}^{[\mathrm{OH}]}\right)(\mathrm{OH})(\mathrm{COOH})$ & 298 & $a_{\mathrm{w}}($ bulk $)$ & 8 & 0.00 & Ganbavale et al. (2014) \\
\hline pyruvic acid & $\mathrm{COOH}, \mathrm{CH}_{\mathrm{n}} \mathrm{CO}$ & $\left(\mathrm{CH}_{3} \mathrm{CO}\right)(\mathrm{COOH})$ & $211-232$ & $a_{\mathrm{w}}\left(T_{\mathrm{hom}}\right)^{\mathrm{a}}$ & 3 & 1.00 & Ganbavale et al. (2014) \\
\hline pyruvic acid & $\mathrm{COOH}, \mathrm{CH}_{\mathrm{n}} \mathrm{CO}$ & $\left(\mathrm{CH}_{3} \mathrm{CO}\right)(\mathrm{COOH})$ & $254-271$ & SLE & 4 & 5.00 & Ganbavale et al. (2014) \\
\hline pyruvic acid & $\mathrm{COOH}, \mathrm{CH}_{n} \mathrm{CO}$ & $\left(\mathrm{CH}_{3} \mathrm{CO}\right)(\mathrm{COOH})$ & 298 & $a_{\mathrm{w}}$ (bulk) & 9 & 0.00 & Ganbavale et al. (2014) \\
\hline 2-methoxyacetic acid & $\mathrm{CH}_{\mathrm{n}}, \mathrm{COOH}, \mathrm{CH}_{\mathrm{n}} \mathrm{O}$ & $\left(\mathrm{CH}_{2}\right)\left(\mathrm{CH}_{3} \mathrm{O}\right)(\mathrm{COOH})$ & $194-232$ & $a_{\mathrm{w}}\left(T_{\mathrm{hom}}\right)^{\mathrm{a}}$ & 5 & 1.00 & Ganbavale et al. (2014) \\
\hline 2-methoxyacetic acid & $\mathrm{CH}_{\mathrm{n}}, \mathrm{COOH}, \mathrm{CH}_{\mathrm{n}} \mathrm{O}$ & $\left(\mathrm{CH}_{2}\right)\left(\mathrm{CH}_{3} \mathrm{O}\right)(\mathrm{COOH})$ & $251-271$ & SLE & 3 & 2.00 & Ganbavale et al. (2014) \\
\hline 2-methoxyacetic acid & $\mathrm{CH}_{\mathrm{n}}, \mathrm{COOH}, \mathrm{CH}_{\mathrm{n}} \mathrm{O}$ & $\left(\mathrm{CH}_{2}\right)\left(\mathrm{CH}_{3} \mathrm{O}\right)(\mathrm{COOH})$ & 268 & $a_{\mathrm{w}}\left(p^{\mathrm{tot}}\right)$ & 4 & 1.00 & Ganbavale et al. (2014) \\
\hline 2-methoxyacetic acid & $\mathrm{CH}_{\mathrm{n}}, \mathrm{COOH}, \mathrm{CH}_{\mathrm{n}} \mathrm{O}$ & $\left(\mathrm{CH}_{2}\right)\left(\mathrm{CH}_{3} \mathrm{O}\right)(\mathrm{COOH})$ & 270 & $a_{\mathrm{w}}\left(p^{\mathrm{tot}}\right)$ & 4 & 1.00 & Ganbavale et al. (2014) \\
\hline 2-methoxyacetic acid & $\mathrm{CH}_{\mathrm{n}}, \mathrm{COOH}, \mathrm{CH}_{\mathrm{n}} \mathrm{O}$ & $\left(\mathrm{CH}_{2}\right)\left(\mathrm{CH}_{3} \mathrm{O}\right)(\mathrm{COOH})$ & 273 & $a_{\mathrm{W}}\left(p^{\mathrm{tot}}\right)$ & 4 & 0.50 & Ganbavale et al. (2014) \\
\hline 2-methoxyacetic acid & $\mathrm{CH}_{\mathrm{n}}, \mathrm{COOH}, \mathrm{CH}_{\mathrm{n}} \mathrm{O}$ & $\left(\mathrm{CH}_{2}\right)\left(\mathrm{CH}_{3} \mathrm{O}\right)(\mathrm{COOH})$ & 275 & $a_{\mathrm{w}}\left(p^{\mathrm{tot}}\right)$ & 5 & 0.50 & Ganbavale et al. (2014) \\
\hline 2-methoxyacetic acid & $\mathrm{CH}_{\mathrm{n}}, \mathrm{COOH}, \mathrm{CH}_{\mathrm{n}} \mathrm{O}$ & $\left(\mathrm{CH}_{2}\right)\left(\mathrm{CH}_{3} \mathrm{O}\right)(\mathrm{COOH})$ & 278 & $a_{\mathrm{w}}\left(p^{\mathrm{tot}}\right)$ & 10 & 1.00 & Ganbavale et al. (2014) \\
\hline 2-methoxyacetic acid & $\mathrm{CH}_{\mathrm{n}}, \mathrm{COOH}, \mathrm{CH}_{\mathrm{n}} \mathrm{O}$ & $\left(\mathrm{CH}_{2}\right)\left(\mathrm{CH}_{3} \mathrm{O}\right)(\mathrm{COOH})$ & 279 & $a_{\mathrm{w}}($ bulk) & 10 & 1.00 & Ganbavale et al. (2014) \\
\hline 2-methoxyacetic acid & $\mathrm{CH}_{\mathrm{n}}, \mathrm{COOH}, \mathrm{CH}_{\mathrm{n}} \mathrm{O}$ & $\left(\mathrm{CH}_{2}\right)\left(\mathrm{CH}_{3} \mathrm{O}\right)(\mathrm{COOH})$ & 280 & $a_{\mathrm{w}}\left(p^{\mathrm{tot}}\right)$ & 10 & 1.00 & Ganbavale et al. (2014) \\
\hline 2-methoxyacetic acid & $\mathrm{CH}_{\mathrm{n}}, \mathrm{COOH}, \mathrm{CH}_{\mathrm{n}} \mathrm{O}$ & $\left(\mathrm{CH}_{2}\right)\left(\mathrm{CH}_{3} \mathrm{O}\right)(\mathrm{COOH})$ & 283 & $a_{\mathrm{W}}\left(p^{\mathrm{tot}}\right)$ & 10 & 1.00 & Ganbavale et al. (2014) \\
\hline 2-methoxyacetic acid & $\mathrm{CH}_{\mathrm{n}}, \mathrm{COOH}, \mathrm{CH}_{\mathrm{n}} \mathrm{O}$ & $\left(\mathrm{CH}_{2}\right)\left(\mathrm{CH}_{3} \mathrm{O}\right)(\mathrm{COOH})$ & 285 & $a_{\mathrm{w}}\left(p^{\mathrm{tot}}\right)$ & 10 & 1.00 & Ganbavale et al. (2014) \\
\hline 2-methoxyacetic acid & $\mathrm{CH}_{\mathrm{n}}, \mathrm{COOH}, \mathrm{CH}_{\mathrm{n}} \mathrm{O}$ & $\left(\mathrm{CH}_{2}\right)\left(\mathrm{CH}_{3} \mathrm{O}\right)(\mathrm{COOH})$ & 288 & $a_{\mathrm{w}}\left(p^{\mathrm{tot}}\right)$ & 10 & 1.00 & Ganbavale et al. (2014) \\
\hline 2-methoxyacetic acid & $\mathrm{CH}_{\mathrm{n}}, \mathrm{COOH}, \mathrm{CH}_{\mathrm{n}} \mathrm{O}$ & $\left(\mathrm{CH}_{2}\right)\left(\mathrm{CH}_{3} \mathrm{O}\right)(\mathrm{COOH})$ & 289 & $a_{\mathrm{w}}($ bulk) & 10 & 0.00 & Ganbavale et al. (2014) \\
\hline 2-methoxyacetic acid & $\mathrm{CH}_{\mathrm{n}}, \mathrm{COOH}, \mathrm{CH}_{\mathrm{n}} \mathrm{O}$ & $\left(\mathrm{CH}_{2}\right)\left(\mathrm{CH}_{3} \mathrm{O}\right)(\mathrm{COOH})$ & 290 & $a_{\mathrm{W}}($ bulk) & 10 & 0.00 & Ganbavale et al. (2014) \\
\hline 2-methoxyacetic acid & $\mathrm{CH}_{\mathrm{n}}, \mathrm{COOH}, \mathrm{CH}_{\mathrm{n}} \mathrm{O}$ & $\left(\mathrm{CH}_{2}\right)\left(\mathrm{CH}_{3} \mathrm{O}\right)(\mathrm{COOH})$ & 298 & $a_{\mathrm{w}}($ bulk) & 9 & 0.00 & Ganbavale et al. (2014) \\
\hline 2-ethoxyethyl acetate & $\mathrm{CH}_{\mathrm{n}}, \mathrm{CH}_{\mathrm{n}} \mathrm{O}, \mathrm{CCOO}$ & $\left(\mathrm{CH}_{3}\right)\left(\mathrm{CH}_{2}\right)\left(\mathrm{CH}_{2} \mathrm{O}\right)_{2}\left(\mathrm{CH}_{3} \mathrm{COO}\right)$ & $208-233$ & $a_{\mathrm{W}}\left(T_{\mathrm{hom}}\right)^{\mathrm{a}}$ & 3 & 1.00 & Ganbavale et al. (2014) \\
\hline 2-ethoxyethyl acetate & $\mathrm{CH}_{\mathrm{n}}, \mathrm{CH}_{\mathrm{n}} \mathrm{O}, \mathrm{CCOO}$ & $\left(\mathrm{CH}_{3}\right)\left(\mathrm{CH}_{2}\right)\left(\mathrm{CH}_{2} \mathrm{O}\right)_{2}\left(\mathrm{CH}_{3} \mathrm{COO}\right)$ & $271-272$ & SLE & 3 & 2.00 & Ganbavale et al. (2014) \\
\hline 2-ethoxyethyl acetate & $\mathrm{CH}_{\mathrm{n}}, \mathrm{CH}_{\mathrm{n}} \mathrm{O}, \mathrm{CCOO}$ & $\left(\mathrm{CH}_{3}\right)\left(\mathrm{CH}_{2}\right)\left(\mathrm{CH}_{2} \mathrm{O}\right)_{2}\left(\mathrm{CH}_{3} \mathrm{COO}\right)$ & $276-368$ & solubli. & 12 & 1.00 & Carvoli and Delogu (1986) \\
\hline resorcinol & $\mathrm{ACH}_{\mathrm{n}}, \mathrm{ACOH}$ & $(\mathrm{ACH})_{4}(\mathrm{ACOH})_{2}$ & $223-232$ & $a_{\mathrm{W}}\left(T_{\mathrm{hom}}\right)^{\mathrm{a}}$ & 4 & 1.00 & Ganbavale et al. (2014) \\
\hline resorcinol & $\mathrm{ACH}_{\mathrm{n}}, \mathrm{ACOH}$ & $(\mathrm{ACH})_{4}(\mathrm{ACOH})_{2}$ & $267-272$ & SLE & 4 & 2.00 & Ganbavale et al. (2014) \\
\hline resorcinol & $\mathrm{ACH}_{\mathrm{n}}, \mathrm{ACOH}$ & $(\mathrm{ACH})_{4}(\mathrm{ACOH})_{2}$ & 298 & $a_{\mathrm{W}}($ bulk) & 7 & 0.00 & Ganbavale et al. (2014) \\
\hline 2-hydroxybenzoic acid & $\mathrm{ACH}_{\mathrm{n}}, \mathrm{ACOH}, \mathrm{COOH}$ & $(\mathrm{ACH})_{4}(\mathrm{AC})(\mathrm{ACOH})(\mathrm{COOH})$ & $298-348$ & SLE(org) ${ }^{\mathrm{d}}$ & 11 & 0.20 & Shalmashi and Eliassi (2008) \\
\hline 2-hydroxybenzoic acid & $\mathrm{ACH}_{\mathrm{n}}, \mathrm{ACOH}, \mathrm{COOH}$ & $(\mathrm{ACH})_{4}(\mathrm{AC})(\mathrm{ACOH})(\mathrm{COOH})$ & $283-339$ & SLE(org) ${ }^{\mathrm{d}}$ & 13 & 0.20 & Apelblat and Manzurola (1989) \\
\hline 2-(2-ethoxyethoxy)ethanol & $\mathrm{CH}_{\mathrm{n}}, \mathrm{CH}_{\mathrm{n}} \mathrm{O}, \mathrm{OH}$ & $\left(\mathrm{CH}_{3}\right)\left(\mathrm{CH}_{2}\right)_{2}\left(\mathrm{CH}_{2} \mathrm{O}\right)_{2}(\mathrm{OH})$ & $211-233$ & $a_{\mathrm{W}}\left(T_{\mathrm{hom}}\right)^{\mathrm{a}}$ & 3 & 0.10 & Ganbavale et al. (2014) \\
\hline 2-(2-ethoxyethoxy)ethanol & $\mathrm{CH}_{\mathrm{n}}, \mathrm{CH}_{\mathrm{n}} \mathrm{O}, \mathrm{OH}$ & $\left(\mathrm{CH}_{3}\right)\left(\mathrm{CH}_{2}\right)_{2}\left(\mathrm{CH}_{2} \mathrm{O}\right)_{2}(\mathrm{OH})$ & $260-272$ & SLE & 4 & 0.10 & Ganbavale et al. (2014) \\
\hline 2-(2-ethoxyethoxy)ethanol & $\mathrm{CH}_{\mathrm{n}}, \mathrm{CH}_{\mathrm{n}} \mathrm{O}, \mathrm{OH}$ & $\left(\mathrm{CH}_{3}\right)\left(\mathrm{CH}_{2}\right)_{2}\left(\mathrm{CH}_{2} \mathrm{O}\right)_{2}(\mathrm{OH})$ & 265 & $a_{\mathrm{w}}\left(p^{\mathrm{tot}}\right)$ & 5 & 0.10 & Ganbavale et al. (2014) \\
\hline 2-(2-ethoxyethoxy)ethanol & $\mathrm{CH}_{\mathrm{n}}, \mathrm{CH}_{\mathrm{n}} \mathrm{O}, \mathrm{OH}$ & $\left(\mathrm{CH}_{3}\right)\left(\mathrm{CH}_{2}\right)_{2}\left(\mathrm{CH}_{2} \mathrm{O}\right)_{2}(\mathrm{OH})$ & 268 & $a_{\mathrm{w}}\left(p^{\mathrm{tot}}\right)$ & 5 & 0.10 & Ganbavale et al. (2014) \\
\hline 2-(2-ethoxyethoxy)ethanol & $\mathrm{CH}_{\mathrm{n}}, \mathrm{CH}_{\mathrm{n}} \mathrm{O}, \mathrm{OH}$ & $\left(\mathrm{CH}_{3}\right)\left(\mathrm{CH}_{2}\right)_{2}\left(\mathrm{CH}_{2} \mathrm{O}\right)_{2}(\mathrm{OH})$ & 270 & $a_{\mathrm{w}}\left(p^{\mathrm{tot}}\right)$ & 5 & 0.10 & Ganbavale et al. (2014) \\
\hline 2-(2-ethoxyethoxy)ethanol & $\mathrm{CH}_{\mathrm{n}}, \mathrm{CH}_{\mathrm{n}} \mathrm{O}, \mathrm{OH}$ & $\left(\mathrm{CH}_{3}\right)\left(\mathrm{CH}_{2}\right)_{2}\left(\mathrm{CH}_{2} \mathrm{O}\right)_{2}(\mathrm{OH})$ & 273 & $a_{\mathrm{w}}\left(p^{\mathrm{tot}}\right)$ & 5 & 0.10 & Ganbavale et al. (2014) \\
\hline 2-(2-ethoxyethoxy)ethanol & $\mathrm{CH}_{\mathrm{n}}, \mathrm{CH}_{\mathrm{n}} \mathrm{O}, \mathrm{OH}$ & $\left(\mathrm{CH}_{3}\right)\left(\mathrm{CH}_{2}\right)_{2}\left(\mathrm{CH}_{2} \mathrm{O}\right)_{2}(\mathrm{OH})$ & 275 & $a_{\mathrm{W}}\left(p^{\mathrm{tot}}\right)$ & 6 & 0.10 & Ganbavale et al. (2014) \\
\hline 2-(2-ethoxyethoxy)ethanol & $\mathrm{CH}_{\mathrm{n}}, \mathrm{CH}_{\mathrm{n}} \mathrm{O}, \mathrm{OH}$ & $\left(\mathrm{CH}_{3}\right)\left(\mathrm{CH}_{2}\right)_{2}\left(\mathrm{CH}_{2} \mathrm{O}\right)_{2}(\mathrm{OH})$ & 278 & $a_{\mathrm{w}}\left(p^{\mathrm{tot}}\right)$ & 9 & 0.10 & Ganbavale et al. (2014) \\
\hline 2-(2-ethoxyethoxy)ethanol & $\mathrm{CH}_{\mathrm{n}}, \mathrm{CH}_{\mathrm{n}} \mathrm{O}, \mathrm{OH}$ & $\left(\mathrm{CH}_{3}\right)\left(\mathrm{CH}_{2}\right)_{2}\left(\mathrm{CH}_{2} \mathrm{O}\right)_{2}(\mathrm{OH})$ & 279 & $a_{\mathrm{w}}($ bulk) & 12 & 0.10 & Ganbavale et al. (2014) \\
\hline 2-(2-ethoxyethoxy)ethanol & $\mathrm{CH}_{\mathrm{n}}, \mathrm{CH}_{\mathrm{n}} \mathrm{O}, \mathrm{OH}$ & $\left(\mathrm{CH}_{3}\right)\left(\mathrm{CH}_{2}\right)_{2}\left(\mathrm{CH}_{2} \mathrm{O}\right)_{2}(\mathrm{OH})$ & 280 & $a_{\mathrm{w}}\left(p^{\mathrm{tot}}\right)$ & 9 & 0.10 & Ganbavale et al. (2014) \\
\hline 2-(2-ethoxyethoxy)ethanol & $\mathrm{CH}_{\mathrm{n}}, \mathrm{CH}_{\mathrm{n}} \mathrm{O}, \mathrm{OH}$ & $\left(\mathrm{CH}_{3}\right)\left(\mathrm{CH}_{2}\right)_{2}\left(\mathrm{CH}_{2} \mathrm{O}\right)_{2}(\mathrm{OH})$ & 283 & $a_{\mathrm{w}}\left(p^{\mathrm{tot}}\right)$ & 9 & 0.10 & Ganbavale et al. (2014) \\
\hline 2-(2-ethoxyethoxy)ethanol & $\mathrm{CH}_{\mathrm{n}}, \mathrm{CH}_{\mathrm{n}} \mathrm{O}, \mathrm{OH}$ & $\left(\mathrm{CH}_{3}\right)\left(\mathrm{CH}_{2}\right)_{2}\left(\mathrm{CH}_{2} \mathrm{O}\right)_{2}(\mathrm{OH})$ & 285 & $a_{\mathrm{w}}\left(p^{\mathrm{tot}}\right)$ & 9 & 0.10 & Ganbavale et al. (2014) \\
\hline 2-(2-ethoxyethoxy)ethanol & $\mathrm{CH}_{\mathrm{n}}, \mathrm{CH}_{\mathrm{n}} \mathrm{O}, \mathrm{OH}$ & $\left(\mathrm{CH}_{3}\right)\left(\mathrm{CH}_{2}\right)_{2}\left(\mathrm{CH}_{2} \mathrm{O}\right)_{2}(\mathrm{OH})$ & 288 & $a_{\mathrm{w}}\left(p^{\mathrm{tot}}\right)$ & 9 & 0.10 & Ganbavale et al. (2014) \\
\hline 2-(2-ethoxyethoxy)ethanol & $\mathrm{CH}_{\mathrm{n}}, \mathrm{CH}_{\mathrm{n}} \mathrm{O}, \mathrm{OH}$ & $\left(\mathrm{CH}_{3}\right)\left(\mathrm{CH}_{2}\right)_{2}\left(\mathrm{CH}_{2} \mathrm{O}\right)_{2}(\mathrm{OH})$ & 289 & $a_{\mathrm{W}}($ bulk) & 12 & 0.00 & Ganbavale et al. (2014) \\
\hline 2-(2-ethoxyethoxy)ethanol & $\mathrm{CH}_{\mathrm{n}}, \mathrm{CH}_{\mathrm{n}} \mathrm{O}, \mathrm{OH}$ & $\left(\mathrm{CH}_{3}\right)\left(\mathrm{CH}_{2}\right)_{2}\left(\mathrm{CH}_{2} \mathrm{O}\right)_{2}(\mathrm{OH})$ & 290 & $a_{\mathrm{w}}($ bulk) & 9 & 0.00 & Ganbavale et al. (2014) \\
\hline 2-(2-ethoxyethoxy)ethanol & $\mathrm{CH}_{\mathrm{n}}, \mathrm{CH}_{\mathrm{n}} \mathrm{O}, \mathrm{OH}$ & $\left(\mathrm{CH}_{3}\right)\left(\mathrm{CH}_{2}\right)_{2}\left(\mathrm{CH}_{2} \mathrm{O}\right)_{2}(\mathrm{OH})$ & 298 & $a_{\mathrm{w}}($ bulk $)$ & 12 & 0.00 & Ganbavale et al. (2014) \\
\hline vanillylmandelic acid & $\begin{array}{l}\mathrm{ACH}_{\mathrm{n}}, \mathrm{ACOH}, \mathrm{CH}_{\mathrm{n}}^{[\mathrm{OH}]}, \\
\mathrm{COOH}, \mathrm{CH}_{\mathrm{n}} \mathrm{O}, \mathrm{OH}\end{array}$ & $\begin{array}{l}(\mathrm{ACH})_{3}(\mathrm{AC})_{2}(\mathrm{ACOH})\left(\mathrm{CH}_{3} \mathrm{O}\right) \\
\left(\mathrm{CH}^{[\mathrm{OH}]}\right)(\mathrm{OH})(\mathrm{COOH})\end{array}$ & 214-232 & $a_{\mathrm{w}}\left(T_{\mathrm{hom}}\right)^{\mathrm{a}}$ & 4 & 1.00 & Zobrist et al. (2008) \\
\hline vanillylmandelic acid & $\begin{array}{l}\mathrm{ACH}_{\mathrm{n}}, \mathrm{ACOH}, \mathrm{CH}_{\mathrm{n}}^{[\mathrm{OH}]} \\
\mathrm{COOH}, \mathrm{CH}_{\mathrm{n}} \mathrm{O}, \mathrm{OH}\end{array}$ & $\begin{array}{l}(\mathrm{ACH})_{3}(\mathrm{AC})_{2}(\mathrm{ACOH})\left(\mathrm{CH}_{3} \mathrm{O}\right) \\
\left(\mathrm{CH}^{[\mathrm{OH}]}\right)(\mathrm{OH})(\mathrm{COOH})\end{array}$ & $267-272$ & SLE & 4 & 5.00 & Zobrist et al. (2008) \\
\hline vanillylmandelic acid & $\begin{array}{l}\mathrm{ACH}_{\mathrm{n}}, \mathrm{ACOH}, \mathrm{CH}_{\mathrm{n}}^{[\mathrm{OH}]}, \\
\mathrm{COOH}, \mathrm{CH}_{\mathrm{n}} \mathrm{O}, \mathrm{OH}\end{array}$ & $\begin{array}{l}(\mathrm{ACH})_{3}(\mathrm{AC})_{2}(\mathrm{ACOH})\left(\mathrm{CH}_{3} \mathrm{O}\right) \\
\left(\mathrm{CH}^{[\mathrm{OH}]}\right)(\mathrm{OH})(\mathrm{COOH})\end{array}$ & 289 & $a_{\mathrm{W}}$ (bulk) & 6 & 0.00 & this work \\
\hline vanillylmandelic acid & $\begin{array}{l}\mathrm{ACH}_{\mathrm{n}}, \mathrm{ACOH}, \mathrm{CH}_{\mathrm{n}}^{[\mathrm{OH}]}, \\
\mathrm{COOH}, \mathrm{CH}_{\mathrm{n}} \mathrm{O}, \mathrm{OH}\end{array}$ & $\begin{array}{l}(\mathrm{ACH})_{3}(\mathrm{AC})_{2}(\mathrm{ACOH})\left(\mathrm{CH}_{3} \mathrm{O}\right) \\
\left(\mathrm{CH}^{[\mathrm{OH}]}\right)(\mathrm{OH})(\mathrm{COOH})\end{array}$ & 298 & $a_{\mathrm{w}}$ (bulk) & 6 & 0.00 & this work \\
\hline vanillylmandelic acid & $\begin{array}{l}\mathrm{ACH}_{\mathrm{n}}, \mathrm{ACOH}, \mathrm{CH}_{\mathrm{n}}^{[\mathrm{OH}]} \\
\mathrm{COOH}, \mathrm{CH}_{\mathrm{n}} \mathrm{O}, \mathrm{OH}\end{array}$ & $\begin{array}{l}(\mathrm{ACH})_{3}(\mathrm{AC})_{2}(\mathrm{ACOH})\left(\mathrm{CH}_{3} \mathrm{O}\right) \\
\left(\mathrm{CH}^{[\mathrm{OH}]}\right)(\mathrm{OH})(\mathrm{COOH})\end{array}$ & 313 & $a_{\mathrm{w}}$ (bulk) & 6 & 1.00 & this work \\
\hline \multicolumn{8}{|c|}{ - water + alcohol + alcohol systems - } \\
\hline 1-butanol, 1-propanol & $\mathrm{CH}_{\mathrm{n}}^{[\mathrm{alc}-\text { tail }]}, \mathrm{CH}_{\mathrm{n}}^{[\mathrm{OH}]}, \mathrm{OH}$ & $\begin{array}{l}\left(\mathrm{CH}_{3}^{[\text {alc-tail }]}\right)\left(\mathrm{CH}_{2}^{[\text {alc-tail }]}\right)_{2}\left(\mathrm{CH}_{2}^{[\mathrm{OH}]}\right)(\mathrm{OH}), \\
\left(\mathrm{CH}_{3}^{[\text {alc-tail }]}\right)\left(\mathrm{CH}_{2}^{[\text {alc-tail }]}\right)\left(\mathrm{CH}_{2}^{[\mathrm{OH}]}\right)(\mathrm{OH})\end{array}$ & 298 & LLE & 20 & 0.00 & Gomis-Yagües et al. (1998) \\
\hline 1-butanol, 1-propanol & $\mathrm{CH}_{\mathrm{n}}^{[\text {alc-tail }]}, \mathrm{CH}_{\mathrm{n}}^{[\mathrm{OH}]}, \mathrm{OH}$ & $\begin{array}{l}\left(\mathrm{CH}_{3}^{[\text {alc-tail }]}\right)\left(\mathrm{CH}_{2}^{\text {[alc-tail }]}\right)_{2}\left(\mathrm{CH}_{2}^{[\mathrm{OH}]}\right)(\mathrm{OH}), \\
\left(\mathrm{CH}_{3}^{[\text {alc-tail }]}\right)\left(\mathrm{CH}_{2}^{[\text {[alc-tail }]}\right)\left(\mathrm{CH}_{2}^{[\mathrm{OH}]}\right)(\mathrm{OH})\end{array}$ & 323 & LLE & 10 & 1.00 & Gomis-Yagües et al. (1998) \\
\hline 1-butanol, 1-propanol & $\mathrm{CH}_{\mathrm{n}}^{[\mathrm{alc}-\text { tail }]}, \mathrm{CH}_{\mathrm{n}}^{[\mathrm{OH}]}, \mathrm{OH}$ & $\begin{array}{l}\left(\mathrm{CH}_{3}^{[\text {alc-tail }]}\right)\left(\mathrm{CH}_{2}^{[\text {alc-tail }]}\right)_{2}\left(\mathrm{CH}_{2}^{[\mathrm{OH}]}\right)(\mathrm{OH}), \\
\left(\mathrm{CH}_{3}^{[\text {alc-tail }]}\right)\left(\mathrm{CH}_{2}^{[\text {alc-tail }]}\right)\left(\mathrm{CH}_{2}^{[\mathrm{OH}]}\right)(\mathrm{OH})\end{array}$ & 358 & LLE & 6 & 1.00 & Gomis-Yagües et al. (1998) \\
\hline 1-butanol, 1-propanol & $\mathrm{CH}_{\mathrm{n}}^{[\text {alc-tail }]}, \mathrm{CH}_{\mathrm{n}}^{[\mathrm{OH}]}, \mathrm{OH}$ & $\begin{array}{l}\left(\mathrm{CH}_{3}^{[\text {alc-tail }]}\right)\left(\mathrm{CH}_{2}^{\text {[alc-tail }]}\right)_{2}\left(\mathrm{CH}_{2}^{[\mathrm{OH}]}\right)(\mathrm{OH}), \\
\left(\mathrm{CH}_{3}^{[\text {alc-tail }]}\right)\left(\mathrm{CH}_{2}^{[\text {alc-tail }]}\right)\left(\mathrm{CH}_{2}^{[\mathrm{OH}]}\right)(\mathrm{OH})\end{array}$ & 368 & LLE & 8 & 1.00 & Gomis-Yagües et al. (1998) \\
\hline 1-pentanol, ethanol & $\mathrm{CH}_{\mathrm{n}}^{[\mathrm{alc}-\text { tail }]}, \mathrm{CH}_{\mathrm{n}}^{[\mathrm{OH}]}, \mathrm{OH}$ & $\begin{array}{l}\left(\mathrm{CH}_{3}^{[\text {alc-tail }]}\right)\left(\mathrm{CH}_{2}^{[\text {[alc-tail }]}\right)_{3}\left(\mathrm{CH}_{2}^{[\mathrm{OH}]}\right)(\mathrm{OH}) \\
\left(\mathrm{CH}_{3}^{[\text {alc-tail }]}\right)\left(\mathrm{CH}_{2}^{[\mathrm{OH}]}\right)(\mathrm{OH})\end{array}$ & 298 & LLE & 12 & 0.00 & Fernández-Torres et al. (1999) \\
\hline 1-pentanol, ethanol & $\mathrm{CH}_{\mathrm{n}}^{[\text {alc-tail }]}, \mathrm{CH}_{\mathrm{n}}^{[\mathrm{OH}]}, \mathrm{OH}$ & $\begin{array}{l}\left(\mathrm{CH}_{3}^{[\text {alc-tail }]}\right)\left(\mathrm{CH}_{2}^{[\text {[alc-tail }]}\right)_{3}\left(\mathrm{CH}_{2}^{[\mathrm{OH}]}\right)(\mathrm{OH}) \\
\left(\mathrm{CH}_{3}^{[\text {[alc-tail }]}\right)\left(\mathrm{CH}_{2}^{[\mathrm{OH}]}\right)(\mathrm{OH})\end{array}$ & 323 & LLE & 12 & 1.00 & Fernández-Torres et al. (1999) \\
\hline
\end{tabular}


Table 1. Continued.

\begin{tabular}{|c|c|c|c|c|c|c|c|}
\hline Organic compounds & Org. main groups & Chemical formula (subgroups) & $T(\mathrm{~K})$ & Data type & $N_{d}$ & $w_{d}^{\text {init }}$ & Reference \\
\hline 1-pentanol, ethanol & $\mathrm{CH}_{\mathrm{n}}^{[\mathrm{alc}-\text { tail }]}, \mathrm{CH}_{\mathrm{n}}^{[\mathrm{OH}]}, \mathrm{OH}$ & $\begin{array}{l}\left(\mathrm{CH}_{3}^{[\text {alc-tail }]}\right)\left(\mathrm{CH}_{2}^{[\text {alc-tail }]}\right)_{3}\left(\mathrm{CH}_{2}^{[\mathrm{OH}]}\right)(\mathrm{OH}) \\
\left(\mathrm{CH}_{3}^{[\text {alc-tail }]}\right)\left(\mathrm{CH}_{2}^{[\mathrm{OH}]}\right)(\mathrm{OH})\end{array}$ & 298 & LLE & 12 & 0.00 & Fernández-Torres et al. (1999) \\
\hline 1-pentanol, ethanol & $\mathrm{CH}_{\mathrm{n}}^{[\text {alc-tail }]}, \mathrm{CH}_{\mathrm{n}}^{[\mathrm{OH}]}, \mathrm{OH}$ & $\begin{array}{l}\left(\mathrm{CH}_{3}^{\text {[alc-tail }]}\right)\left(\mathrm{CH}_{2}^{\text {[alc-tail }]}\right)_{3}\left(\mathrm{CH}_{2}^{[\mathrm{OH}]}\right)(\mathrm{OH}) \\
\left(\mathrm{CH}_{3}^{[\text {alc-tail }]}\right)\left(\mathrm{CH}_{2}^{[\mathrm{OH}]}\right)(\mathrm{OH})\end{array}$ & 323 & LLE & 12 & 1.00 & Fernández-Torres et al. (1999) \\
\hline 1-pentanol, ethanol & $\mathrm{CH}_{\mathrm{n}}^{[\text {alc-tail }]}, \mathrm{CH}_{\mathrm{n}}^{[\mathrm{OH}]}, \mathrm{OH}$ & $\begin{array}{l}\left(\mathrm{CH}_{3}^{\text {[alc-tail] }}\right)\left(\mathrm{CH}_{2}^{\text {[alc-tail }]}\right)_{3}\left(\mathrm{CH}_{2}^{[\mathrm{OH}]}\right)(\mathrm{OH}) \\
\left(\mathrm{CH}_{3}^{[\text {alc-tail }]}\right)\left(\mathrm{CH}_{2}^{[\mathrm{OH}]}\right)(\mathrm{OH})\end{array}$ & 358 & LLE & 8 & 1.00 & Fernández-Torres et al. (1999) \\
\hline 1-pentanol, ethanol & $\mathrm{CH}_{\mathrm{n}}^{[\text {alc-tail }]}, \mathrm{CH}_{\mathrm{n}}^{[\mathrm{OH}]}, \mathrm{OH}$ & $\begin{array}{l}\left(\mathrm{CH}_{3}^{\text {[alc-tail }]}\right)\left(\mathrm{CH}_{2}^{\text {[alc-tail }]}\right)_{3}\left(\mathrm{CH}_{2}^{[\mathrm{OH}]}\right)(\mathrm{OH}) \\
\left(\mathrm{CH}_{3}^{[\text {alc-tail] }}\right)\left(\mathrm{CH}_{2}^{[\mathrm{OH}]}\right)(\mathrm{OH})\end{array}$ & 368 & LLE & 12 & 1.00 & Fernández-Torres et al. (1999) \\
\hline \multicolumn{8}{|c|}{ - water + alcohol + acid systems - } \\
\hline 1-butanol, acetic acid & $\begin{array}{l}\mathrm{CH}_{\mathrm{n}}, \mathrm{CH}_{\mathrm{n}}^{[\text {alc-tail }]}, \mathrm{CH}_{\mathrm{n}}^{[\mathrm{OH}]}, \mathrm{OH}, \\
\mathrm{COOH}\end{array}$ & $\begin{array}{l}\left(\mathrm{CH}_{3}^{[\text {alc-tail }]}\right)\left(\mathrm{CH}_{2}^{[\text {alc-tail }]}\right)_{2}\left(\mathrm{CH}_{2}^{[\mathrm{OH}]}\right)(\mathrm{OH}) \\
\left(\mathrm{CH}_{3}\right)(\mathrm{COOH})\end{array}$ & 298 & LLE & 10 & 0.00 & Ruiz Bevia et al. (1984) \\
\hline 1-butanol, acetic acid & $\begin{array}{l}\mathrm{CH}_{\mathrm{n}}, \mathrm{CH}_{\mathrm{n}}^{[\text {alc-tail }]}, \mathrm{CH}_{\mathrm{n}}^{[\mathrm{OH}]}, \mathrm{OH} \\
\mathrm{COOH}\end{array}$ & $\begin{array}{l}\left(\mathrm{CH}_{3}^{[\text {alc-tail }]}\right)\left(\mathrm{CH}_{2}^{[\text {alc-tail }]}\right)_{2}\left(\mathrm{CH}_{2}^{[\mathrm{OH}]}\right)(\mathrm{OH}) \\
\left(\mathrm{CH}_{3}\right)(\mathrm{COOH})\end{array}$ & 303 & LLE & 12 & 0.00 & Esquível and Bernardo-Gil (1990) \\
\hline 1-butanol, acetic acid & $\begin{array}{l}\mathrm{CH}_{\mathrm{n}}, \mathrm{CH}_{\mathrm{n}}^{[\text {alc-tail }]}, \mathrm{CH}_{\mathrm{n}}^{[\mathrm{OH}]}, \mathrm{OH} \\
\mathrm{COOH}\end{array}$ & $\begin{array}{l}\left(\mathrm{CH}_{3}^{\text {[alc-tail }]}\right)\left(\mathrm{CH}_{2}^{[\text {alc-tail }]}\right)_{2}\left(\mathrm{CH}_{2}^{[\mathrm{OH}]}\right)(\mathrm{OH}), \\
\left(\mathrm{CH}_{3}\right)(\mathrm{COOH})\end{array}$ & 323 & LLE & 14 & 1.00 & Esquível and Bernardo-Gil (1990) \\
\hline 2-butanol, acetic acid & $\begin{array}{l}\mathrm{CH}_{\mathrm{n}}, \mathrm{CH}_{\mathrm{n}}^{[\text {alc-tail }]}, \mathrm{CH}_{\mathrm{n}}^{[\mathrm{OH}]}, \mathrm{OH} \\
\mathrm{COOH}\end{array}$ & $\begin{array}{l}\left(\mathrm{CH}_{3}^{[\text {alc }]}\right)\left(\mathrm{CH}_{3}^{[\text {alc-tail }]}\right) \\
\left(\mathrm{CH}_{2}^{[\text {alc-tail }]}\right)\left(\mathrm{CH}^{[\mathrm{OH}]}\right)(\mathrm{OH}),\left(\mathrm{CH}_{3}\right)(\mathrm{COOH})\end{array}$ & 303 & LLE & 10 & 0.00 & Esquível and Bernardo-Gil (1990) \\
\hline 2-butanol, acetic acid & $\begin{array}{l}\mathrm{CH}_{\mathrm{n}}, \mathrm{CH}_{\mathrm{n}}^{[\text {alc-tail }]}, \mathrm{CH}_{\mathrm{n}}^{[\mathrm{OH}]}, \mathrm{OH}, \\
\mathrm{COOH}\end{array}$ & $\begin{array}{l}\left(\mathrm{CH}_{3}^{[\text {alc }]}\right)\left(\mathrm{CH}_{3}^{\text {[alc-tail] }}\right) \\
\left(\mathrm{CH}_{2}^{[\text {alc-tail }]}\right)\left(\mathrm{CH}^{[\mathrm{OH}]}\right)(\mathrm{OH}),\left(\mathrm{CH}_{3}\right)(\mathrm{COOH})\end{array}$ & 323 & LLE & 14 & 1.00 & Esquível and Bernardo-Gil (1990) \\
\hline 1-butanol, propanoic acid & $\begin{array}{l}\mathrm{CH}_{\mathrm{n}}, \mathrm{CH}_{\mathrm{n}}^{[\text {alc-tail }]}, \mathrm{CH}_{\mathrm{n}}^{[\mathrm{OH}]}, \mathrm{OH}, \\
\mathrm{COOH}\end{array}$ & $\begin{array}{l}\left(\mathrm{CH}_{3}^{\text {alc-tail }]}\right)\left(\mathrm{CH}_{2}^{[\text {alc-tail }]}\right)_{2}\left(\mathrm{CH}_{2}^{[\mathrm{OH}]}\right)(\mathrm{OH}), \\
\left(\mathrm{CH}_{3}\right)\left(\mathrm{CH}_{2}\right)(\mathrm{COOH})\end{array}$ & 298 & LLE & 8 & 0.00 & Kim and Park (2005) \\
\hline 1-butanol, propanoic acid & $\begin{array}{l}\mathrm{CH}_{\mathrm{n}}, \mathrm{CH}_{\mathrm{n}}^{[\text {alc-tail }]}, \mathrm{CH}_{\mathrm{n}}^{[\mathrm{OH}]}, \mathrm{OH} \\
\mathrm{COOH}\end{array}$ & $\begin{array}{l}\left(\mathrm{CH}_{3}^{\text {[alc-tail }]}\right)\left(\mathrm{CH}_{2}^{[\text {alc-tail }]}\right)_{2}\left(\mathrm{CH}_{2}^{[\mathrm{OH}]}\right)(\mathrm{OH}), \\
\left(\mathrm{CH}_{3}\right)\left(\mathrm{CH}_{2}\right)(\mathrm{COOH})\end{array}$ & 303 & LLE & 14 & 0.00 & Sólimo et al. (1997) \\
\hline 2-butanol, citric acid & $\begin{array}{l}\mathrm{CH}_{\mathrm{n}}, \mathrm{CH}_{\mathrm{n}}^{[\text {alc }]}, \quad \mathrm{CH}_{\mathrm{n}}^{[\text {alc-tail }]}, \\
\mathrm{CH}_{\mathrm{n}}^{[\mathrm{OH}]}, \mathrm{OH}, \mathrm{COOH}\end{array}$ & $\begin{array}{l}\left(\mathrm{CH}_{3}^{\text {[alc }]}\right)\left(\mathrm{CH}_{3}^{\text {[alc-tail }]}\right) \\
\left(\mathrm{CH}_{2}^{\text {[alc-tail] }}\right)\left(\mathrm{CH}^{[\mathrm{OH}]}\right)(\mathrm{OH}) \\
\left(\mathrm{CH}_{2}\right)_{2}\left(\mathrm{C}^{[\mathrm{OH}]}\right)(\mathrm{COOH})_{3}(\mathrm{OH})\end{array}$ & 298 & LLE & 8 & 0.00 & Lintomen et al. (2001) \\
\hline 2-pentanol, acetic acid & $\begin{array}{l}\mathrm{CH}_{\mathrm{n}}, \quad \mathrm{CH}_{\mathrm{n}}^{[\text {alc }]}, \quad \mathrm{CH}_{\mathrm{n}}^{[\text {alc }- \text { tail }]}, \\
\mathrm{CH}_{\mathrm{n}}^{[\mathrm{OH}]}, \mathrm{OH}, \mathrm{COOH}\end{array}$ & $\begin{array}{l}\left(\mathrm{CH}_{3}^{\text {[alc }]}\right)\left(\mathrm{CH}_{3}^{\text {[alc-tail] }}\right) \\
\left(\mathrm{CH}_{2}^{\text {[alc-tail] }}\right)_{2}\left(\mathrm{CH}^{[\mathrm{OH}]}\right)(\mathrm{OH}),\left(\mathrm{CH}_{3}\right)(\mathrm{COOH})\end{array}$ & 288 & LLE & 20 & 0.20 & Al-Muhtaseb and Fahim (1996) \\
\hline 2-pentanol, acetic acid & $\begin{array}{l}\mathrm{CH}_{\mathrm{n}}, \quad \mathrm{CH}_{\mathrm{n}}^{[\text {alc }]}, \quad \mathrm{CH}_{\mathrm{n}}^{[\text {alc-tail }]}, \\
\mathrm{CH}_{\mathrm{n}}^{[\mathrm{OH}]}, \mathrm{OH}, \mathrm{COOH}\end{array}$ & $\begin{array}{l}\left(\mathrm{CH}_{3}^{[\text {alc }]}\right)\left(\mathrm{CH}_{3}^{\text {[alc-tail }]}\right) \\
\left(\mathrm{CH}_{2}^{\text {[alc-tail] }}\right)_{2}\left(\mathrm{CH}^{[\mathrm{OH}]}\right)(\mathrm{OH}),\left(\mathrm{CH}_{3}\right)(\mathrm{COOH})\end{array}$ & 298 & LLE & 20 & 0.00 & Al-Muhtaseb and Fahim (1996) \\
\hline 2-pentanol, acetic acid & $\begin{array}{l}\mathrm{CH}_{\mathrm{n}}, \mathrm{CH}_{\mathrm{n}}^{[\text {alc }]}, \mathrm{CH}_{\mathrm{n}}^{[\text {[alc-tail }]}, \\
\mathrm{CH}_{\mathrm{n}}^{[\mathrm{OH}]}, \mathrm{OH}, \mathrm{COOH}\end{array}$ & $\begin{array}{l}\left(\mathrm{CH}_{3}^{[\text {alc }]}\right)\left(\mathrm{CH}_{3}^{\text {[alc-tail }]}\right) \\
\left(\mathrm{CH}_{2}^{\text {[alc-tail] }}\right)_{2}\left(\mathrm{CH}^{[\mathrm{OH}]}\right)(\mathrm{OH}),\left(\mathrm{CH}_{3}\right)(\mathrm{COOH})\end{array}$ & 303 & LLE & 8 & 0.00 & Al-Muhtaseb and Fahim (1996) \\
\hline 2-pentanol, acetic acid & $\begin{array}{l}\mathrm{CH}_{\mathrm{n}}, \quad \mathrm{CH}_{\mathrm{n}}^{[\text {alc }]}, \quad \mathrm{CH}_{\mathrm{n}}^{[\text {alc-tail }]}, \\
\mathrm{CH}_{\mathrm{n}}^{[\mathrm{OH}]}, \mathrm{OH}, \mathrm{COOH}\end{array}$ & $\begin{array}{l}\left(\mathrm{CH}_{3}^{[\text {alc }]}\right)\left(\mathrm{CH}_{3}^{\text {[alc-tail] }}\right) \\
\left(\mathrm{CH}_{2}^{[\text {alc-tail] }}\right)_{2}\left(\mathrm{CH}^{[\mathrm{OH}]}\right)(\mathrm{OH}),\left(\mathrm{CH}_{3}\right)(\mathrm{COOH})\end{array}$ & 308 & LLE & 10 & 1.00 & Al-Muhtaseb and Fahim (1996) \\
\hline 2-pentanol, acetic acid & $\begin{array}{l}\mathrm{CH}_{\mathrm{n}}, \quad \mathrm{CH}_{\mathrm{n}}^{\text {[alc] }}, \quad \mathrm{CH}_{\mathrm{n}}^{\text {[alc-tail] }}, \\
\mathrm{CH}_{\mathrm{n}}^{[\mathrm{[H}]}, \mathrm{OH}, \mathrm{COOH}\end{array}$ & $\begin{array}{l}\left(\mathrm{CH}_{3}^{\text {[alc }]}\right)\left(\mathrm{CH}_{3}^{\text {[alc-tail }]}\right)\left(\mathrm{CH}_{2}^{[\text {alc-tail }]}\right)_{2} \\
\left(\mathrm{CH}^{[\mathrm{OH}]}\right)(\mathrm{OH}),\left(\mathrm{CH}_{3}\right)(\mathrm{COOH})\end{array}$ & 318 & LLE & 8 & 1.00 & Al-Muhtaseb and Fahim (1996) \\
\hline 2-pentanol, acetic acid & $\begin{array}{l}\mathrm{CH}_{\mathrm{n}}, \mathrm{CH}_{\mathrm{n}}^{[\text {alc }]}, \quad \mathrm{CH}_{\mathrm{n}}^{[\text {alc-tail }]}, \\
\mathrm{CH}_{\mathrm{n}}^{[\mathrm{OH}]}, \mathrm{OH}, \mathrm{COOH}\end{array}$ & $\begin{array}{l}\left(\mathrm{CH}_{3}^{[\text {alc }]}\right)\left(\mathrm{CH}_{3}^{\text {[alc-tail }]}\right) \\
\left(\mathrm{CH}_{2}^{[\text {alc-tail] }}\right)_{2}\left(\mathrm{CH}^{[\mathrm{OH}]}\right)(\mathrm{OH}),\left(\mathrm{CH}_{3}\right)(\mathrm{COOH})\end{array}$ & 323 & LLE & 14 & 1.00 & Al-Muhtaseb and Fahim (1996) \\
\hline 1-hexanol, acetic acid & $\begin{array}{l}\mathrm{CH}_{\mathrm{n}}, \mathrm{CH}_{\mathrm{n}}^{[\mathrm{OH}]}, \mathrm{CH}_{\mathrm{n}}^{[\text {alc-tail }]}, \mathrm{OH} \\
\mathrm{COOH}\end{array}$ & $\begin{array}{l}\left(\mathrm{CH}_{3}^{\text {[alc-tail }]}\right)\left(\mathrm{CH}_{2}^{[\text {alc-tail }]}\right)_{4}\left(\mathrm{CH}_{2}^{[\mathrm{OH}]}\right)(\mathrm{OH}) \\
\left(\mathrm{CH}_{3}\right)(\mathrm{COOH})\end{array}$ & 293 & LLE & 8 & 0.00 & Senol (2004) \\
\hline \multicolumn{8}{|c|}{ - water + alcohol + ketone systems - } \\
\hline $\begin{array}{l}\text { tert-butanol, 4-methyl-2- } \\
\text { pentanone }\end{array}$ & $\begin{array}{l}\mathrm{CH}_{\mathrm{n}}, \mathrm{CH}_{\mathrm{n}}^{[\mathrm{alc}]}, \mathrm{CH}_{\mathrm{n}}^{[\mathrm{OH}]}, \mathrm{OH}, \\
\mathrm{CH}_{\mathrm{n}} \mathrm{CO}\end{array}$ & $\begin{array}{l}\left(\mathrm{CH}_{3}^{[\mathrm{alc}]}\right)_{3}\left(\mathrm{C}^{[\mathrm{OH}]}\right)(\mathrm{OH}) \\
\left(\mathrm{CH}_{3}\right)_{2}\left(\mathrm{CH}_{2}\right)(\mathrm{CH})\left(\mathrm{CH}_{3} \mathrm{CO}\right)\end{array}$ & 288 & LLE & 14 & 0.10 & Fang et al. (2008) \\
\hline $\begin{array}{l}\text { tert-butanol, 4-methyl-2- } \\
\text { pentanone }\end{array}$ & $\begin{array}{l}\mathrm{CH}_{\mathrm{n}}, \mathrm{CH}_{\mathrm{n}}^{[\mathrm{alc}]}, \mathrm{CH}_{\mathrm{n}}^{[\mathrm{OH}]}, \mathrm{OH}, \\
\mathrm{CH}_{\mathrm{n}} \mathrm{CO}\end{array}$ & $\begin{array}{l}\left(\mathrm{CH}_{3}^{[\mathrm{alc}]}\right)_{3}\left(\mathrm{C}^{[\mathrm{OH}]}\right)(\mathrm{OH}) \\
\left(\mathrm{CH}_{3}\right)_{2}\left(\mathrm{CH}_{2}\right)(\mathrm{CH})\left(\mathrm{CH}_{3} \mathrm{CO}\right)\end{array}$ & 304 & LLE & 16 & 0.00 & Fang et al. (2008) \\
\hline $\begin{array}{l}\text { tert-butanol, 4-methyl-2- } \\
\text { pentanone }\end{array}$ & $\begin{array}{l}\mathrm{CH}_{\mathrm{n}}, \mathrm{CH}_{\mathrm{n}}^{[\mathrm{alc}]}, \mathrm{CH}_{\mathrm{n}}^{[\mathrm{OH}]}, \mathrm{OH}, \\
\mathrm{CH}_{\mathrm{n}} \mathrm{CO}\end{array}$ & $\begin{array}{l}\left(\mathrm{CH}_{3}^{\text {[alc] }}\right)_{3}\left(\mathrm{C}^{[\mathrm{OH}]}\right)(\mathrm{OH}) \\
\left(\mathrm{CH}_{3}\right)_{2}\left(\mathrm{CH}_{2}\right)(\mathrm{CH})\left(\mathrm{CH}_{3} \mathrm{CO}\right)\end{array}$ & 318 & LLE & 18 & 0.10 & Fang et al. (2008) \\
\hline $\begin{array}{l}\text { tert-butanol, 4-methyl-2- } \\
\text { pentanone }\end{array}$ & $\begin{array}{l}\mathrm{CH}_{\mathrm{n}}, \mathrm{CH}_{\mathrm{n}}^{[\mathrm{alc}]}, \mathrm{CH}_{\mathrm{n}}^{[\mathrm{OH}]}, \mathrm{OH} \\
\mathrm{CH}_{\mathrm{n}} \mathrm{CO}\end{array}$ & $\begin{array}{l}\left(\mathrm{CH}_{3}^{[\mathrm{ialc}]}\right)_{3}\left(\mathrm{C}^{[\mathrm{OH}]}\right)(\mathrm{OH}) \\
\left(\mathrm{CH}_{3}\right)_{2}\left(\mathrm{CH}_{2}\right)(\mathrm{CH})\left(\mathrm{CH}_{3} \mathrm{CO}\right)\end{array}$ & 333 & LLE & 16 & 0.10 & Fang et al. (2008) \\
\hline 1-pentanol, acetone & $\begin{array}{l}\mathrm{CH}_{\mathrm{n}}, \mathrm{CH}_{\mathrm{n}}^{[\text {alc-tail }]}, \mathrm{CH}_{\mathrm{n}}^{[\mathrm{OH}]}, \mathrm{OH} \\
\mathrm{CH}_{\mathrm{n}} \mathrm{CO}\end{array}$ & $\begin{array}{l}\left(\mathrm{CH}_{3}^{\text {[alc-tail }]}\right)\left(\mathrm{CH}_{2}^{[\text {alc-tail }]}\right)_{3}\left(\mathrm{CH}_{2}^{[\mathrm{OH}]}\right)(\mathrm{OH}), \\
\left(\mathrm{CH}_{3}\right)\left(\mathrm{CH}_{3} \mathrm{CO}\right)\end{array}$ & 298 & LLE & 16 & 0.00 & Tiryaki et al. (1994) \\
\hline 1-pentanol, acetone & $\begin{array}{l}\mathrm{CH}_{\mathrm{n}}, \mathrm{CH}_{\mathrm{n}}^{[\text {alc-tail }]}, \mathrm{CH}_{\mathrm{n}}^{[\mathrm{OH}]}, \mathrm{OH}, \\
\mathrm{CH}_{\mathrm{n}} \mathrm{CO}\end{array}$ & $\begin{array}{l}\left(\mathrm{CH}_{3}^{\text {alc-tail }]}\right)\left(\mathrm{CH}_{2}^{[\text {alc-tail }]}\right)_{3}\left(\mathrm{CH}_{2}^{[\mathrm{OH}]}\right)(\mathrm{OH}), \\
\left(\mathrm{CH}_{3}\right)\left(\mathrm{CH}_{3} \mathrm{CO}\right)\end{array}$ & 303 & LLE & 16 & 0.00 & Tiryaki et al. (1994) \\
\hline 1-pentanol, acetone & $\begin{array}{l}\mathrm{CH}_{\mathrm{n}}, \mathrm{CH}_{\mathrm{n}}^{[\text {alc-tail] }}, \mathrm{CH}_{\mathrm{n}}^{[\mathrm{OH}]}, \mathrm{OH}, \\
\mathrm{CH}_{\mathrm{n}} \mathrm{CO}\end{array}$ & $\begin{array}{l}\left(\mathrm{CH}_{3}^{\text {[alc-tail }]}\right)\left(\mathrm{CH}_{2}^{[\text {alc-tail })_{3}}\left(\mathrm{CH}_{2}^{[\mathrm{OH}]}\right)(\mathrm{OH})\right. \\
\left(\mathrm{CH}_{3}\right)\left(\mathrm{CH}_{3} \mathrm{CO}\right)\end{array}$ & 308 & LLE & 16 & 0.00 & Tiryaki et al. (1994) \\
\hline 2-octanol, acetone & $\begin{array}{ll}\mathrm{CH}_{\mathrm{n}}, & \mathrm{CH}_{\mathrm{n}}^{[\text {alc-tail }]}, \\
\mathrm{CH}_{\mathrm{n}}^{[\mathrm{OH}]}, \mathrm{OH}, \mathrm{CH}_{\mathrm{n}} \mathrm{CO}\end{array}$ & $\begin{array}{l}\left(\mathrm{CH}_{3}^{\text {[alc-tail] }}\right)\left(\mathrm{CH}_{3}^{[\text {alc] }}\right) \\
\left(\mathrm{CH}_{2}^{\text {[alc-tail }]}\right)_{5}\left(\mathrm{CH}^{[\mathrm{OH}]}\right)(\mathrm{OH}),\left(\mathrm{CH}_{3}\right)\left(\mathrm{CH}_{3} \mathrm{CO}\right)\end{array}$ & 298 & LLE & 18 & 0.00 & Tiryaki et al. (1994) \\
\hline 2-octanol, acetone & $\begin{array}{ll}\mathrm{CH}_{\mathrm{n}}, & \mathrm{CH}_{\mathrm{n}}^{\text {[alc-tail }]}, \\
\mathrm{CH}_{\mathrm{n}}^{[\mathrm{OH}]}, \mathrm{OH}, \mathrm{CH}_{\mathrm{n}} \mathrm{CO}\end{array}$ & $\begin{array}{l}\left(\mathrm{CH}_{3}^{\text {[alc-tail }]}\right)\left(\mathrm{CH}_{3}^{[\text {alc }]}\right) \\
\left(\mathrm{CH}_{2}^{[\text {alc-tail }]}\right)_{5}\left(\mathrm{CH}^{[\mathrm{OH}]}\right)(\mathrm{OH}),\left(\mathrm{CH}_{3}\right)\left(\mathrm{CH}_{3} \mathrm{CO}\right)\end{array}$ & 303 & LLE & 18 & 0.00 & Tiryaki et al. (1994) \\
\hline 2-octanol, acetone & $\begin{array}{l}\mathrm{CH}_{\mathrm{n}}, \quad \mathrm{CH}_{\mathrm{n}}^{[\text {[alc-tail }]}, \quad \mathrm{CH}_{\mathrm{n}}^{[\text {alc }]}, \\
\mathrm{CH}_{\mathrm{n}}^{[\mathrm{OH}]}, \mathrm{OH}, \mathrm{CH}_{\mathrm{n}} \mathrm{CO}\end{array}$ & $\begin{array}{l}\left(\mathrm{CH}_{3}^{\text {[alc-tail }]}\right)\left(\mathrm{CH}_{3}^{[\text {alc }]}\right) \\
\left(\mathrm{CH}_{2}^{[\text {alc-tail }]}\right)_{5}\left(\mathrm{CH}^{[\mathrm{OH}]}\right)(\mathrm{OH}),\left(\mathrm{CH}_{3}\right)\left(\mathrm{CH}_{3} \mathrm{CO}\right)\end{array}$ & 308 & LLE & 16 & 1.00 & Tiryaki et al. (1994) \\
\hline \multicolumn{8}{|c|}{ - water + alcohol + ether systems - } \\
\hline $\begin{array}{l}\text { ethanol, 2-ethoxy-2- } \\
\text { methyl-propane }\end{array}$ & $\begin{array}{l}\mathrm{CH}_{\mathrm{n}}, \mathrm{CH}_{\mathrm{n}}^{[\text {[alc-tail }]}, \mathrm{CH}_{\mathrm{n}}^{[\mathrm{OH}]}, \mathrm{OH}, \\
\mathrm{CH}_{\mathrm{n}} \mathrm{O}\end{array}$ & $\left(\mathrm{CH}_{3}^{[\text {alc-tail] }}\right)\left(\mathrm{CH}_{2}^{[\mathrm{OH}]}\right)(\mathrm{OH}),\left(\mathrm{CH}_{3}\right)_{4}(\mathrm{C})\left(\mathrm{CH}_{2} \mathrm{O}\right)$ & 288 & LLE & 14 & 0.20 & Fandary et al. (1999) \\
\hline $\begin{array}{l}\text { ethanol, 2-ethoxy-2- } \\
\text { methyl-propane }\end{array}$ & $\begin{array}{l}\mathrm{CH}_{\mathrm{n}}, \mathrm{CH}_{\mathrm{n}}^{[\text {[alc-tail }]}, \mathrm{CH}_{\mathrm{n}}^{[\mathrm{OH}]}, \mathrm{OH}, \\
\mathrm{CH}_{\mathrm{n}} \mathrm{O}\end{array}$ & $\left(\mathrm{CH}_{3}^{[\text {alc-tail }]}\right)\left(\mathrm{CH}_{2}^{[\mathrm{OH}]}\right)(\mathrm{OH}),\left(\mathrm{CH}_{3}\right)_{4}(\mathrm{C})\left(\mathrm{CH}_{2} \mathrm{O}\right)$ & 298 & LLE & 14 & 0.00 & Fandary et al. (1999) \\
\hline $\begin{array}{l}\text { exthanol, 2-ethoxy-2- } \\
\text { methyl-propane }\end{array}$ & $\begin{array}{l}\mathrm{CH}_{\mathrm{n}}, \mathrm{CH}_{\mathrm{n}}^{[\text {[alc-tail }]}, \mathrm{CH}_{\mathrm{n}}^{[\mathrm{OH}]}, \mathrm{OH}, \\
\mathrm{CH}_{\mathrm{n}} \mathrm{O}\end{array}$ & $\left(\mathrm{CH}_{3}^{[\text {alc-tail }]}\right)\left(\mathrm{CH}_{2}^{[\mathrm{OH}]}\right)(\mathrm{OH}),\left(\mathrm{CH}_{3}\right)_{4}(\mathrm{C})\left(\mathrm{CH}_{2} \mathrm{O}\right)$ & 303 & LLE & 14 & 0.00 & Fandary et al. (1999) \\
\hline
\end{tabular}


Table 1. Continued.

\begin{tabular}{|c|c|c|c|c|c|c|c|}
\hline Organic compounds & \multirow{2}{*}{$\begin{array}{l}\text { Org. main groups } \\
\mathrm{CH}_{\mathrm{n}}, \mathrm{CH}_{\mathrm{n}}^{[\mathrm{alc}-\text { tail }]}, \mathrm{CH}_{\mathrm{n}}^{[\mathrm{OH}]}, \mathrm{OH}, \\
\mathrm{CH}_{\mathrm{n}} \mathrm{O}\end{array}$} & \multirow{2}{*}{$\begin{array}{l}\text { Chemical formula (subgroups) } \\
\left(\mathrm{CH}_{3}^{[\text {alc-tail }]}\right)\left(\mathrm{CH}_{2}^{[\mathrm{OH}]}\right)(\mathrm{OH}),\left(\mathrm{CH}_{3}\right)_{4}(\mathrm{C})\left(\mathrm{CH}_{2} \mathrm{O}\right)\end{array}$} & \multirow{2}{*}{$\frac{T(\mathrm{~K})}{308}$} & \multirow{2}{*}{$\begin{array}{l}\text { Data type } \\
\text { LLE }\end{array}$} & \multirow{2}{*}{$\frac{N_{d}}{14}$} & \multirow{2}{*}{$\frac{w_{d}^{\text {init }}}{0.00}$} & \multirow{2}{*}{$\begin{array}{l}\text { Reference } \\
\text { Fandary et al. (1999) }\end{array}$} \\
\hline $\begin{array}{l}\text { ethanol, 2-ethoxy-2- } \\
\text { methyl-propane }\end{array}$ & & & & & & & \\
\hline \multicolumn{8}{|c|}{ - water + alcohol + ester systems - } \\
\hline ethanol, ethyl acetate & $\begin{array}{l}\mathrm{CH}_{\mathrm{n}}, \mathrm{CH}_{\mathrm{n}}^{[\text {alc-tail }]}, \mathrm{CH}_{\mathrm{n}}^{[\mathrm{OH}]}, \mathrm{OH} \\
\mathrm{CCOO}\end{array}$ & $\begin{array}{l}\left(\mathrm{CH}_{3}^{[\text {alc-tail }]}\right)\left(\mathrm{CH}_{2}^{[\mathrm{OH}]}\right)(\mathrm{OH}) \\
\left(\mathrm{CH}_{3}\right)\left(\mathrm{CH}_{2}\right)\left(\mathrm{CH}_{3} \mathrm{COO}\right)\end{array}$ & 313 & LLE & 10 & 1.00 & Mertl (1972) \\
\hline ethanol, ethyl acetate & $\begin{array}{l}\mathrm{CH}_{\mathrm{n}}, \mathrm{CH}_{\mathrm{n}}^{[\text {alc-tail }]}, \mathrm{CH}_{\mathrm{n}}^{[\mathrm{OH}]}, \mathrm{OH} \\
\mathrm{CCOO}\end{array}$ & $\begin{array}{l}\left(\mathrm{CH}_{3}^{[\text {alc-tail] }}\right)\left(\mathrm{CH}_{2}^{[\mathrm{OH}]}\right)(\mathrm{OH}) \\
\left(\mathrm{CH}_{3}\right)\left(\mathrm{CH}_{2}\right)\left(\mathrm{CH}_{3} \mathrm{COO}\right)\end{array}$ & 328 & LLE & 10 & 1.00 & Mertl (1972) \\
\hline ethanol, ethyl acetate & $\begin{array}{l}\mathrm{CH}_{\mathrm{n}}, \mathrm{CH}_{\mathrm{n}}^{[\mathrm{alc}-\text { tail }]}, \mathrm{CH}_{\mathrm{n}}^{[\mathrm{OH}]}, \mathrm{OH} \\
\mathrm{CCOO}\end{array}$ & $\begin{array}{l}\left(\mathrm{CH}_{3}^{[\text {alc-tail }]}\right)\left(\mathrm{CH}_{2}^{[\mathrm{OH}]}\right)(\mathrm{OH}) \\
\left(\mathrm{CH}_{3}\right)\left(\mathrm{CH}_{2}\right)\left(\mathrm{CH}_{3} \mathrm{COO}\right)\end{array}$ & 343 & LLE & 10 & 1.00 & Mertl (1972) \\
\hline \multicolumn{8}{|c|}{ - water + alcohol + aromatic systems - } \\
\hline 1-butanol, phenol & $\begin{array}{l}\mathrm{CH}_{\mathrm{n}}^{[\text {alc-tail }]}, \mathrm{CH}_{\mathrm{n}}^{[\mathrm{OH}]}, \mathrm{OH} \\
\mathrm{ACH}_{\mathrm{n}}, \mathrm{ACOH}\end{array}$ & $\begin{array}{l}\left(\mathrm{CH}_{3}^{[\text {alc-tail }]}\right)\left(\mathrm{CH}_{2}^{[\text {alc-tail }]}\right)_{2}\left(\mathrm{CH}_{2}^{[\mathrm{OH}]}\right)(\mathrm{OH}), \\
(\mathrm{ACH})_{5}(\mathrm{ACOH})\end{array}$ & 298 & LLE & 12 & 0.00 & De Oliveira and Aznar (2010) \\
\hline 2-butanol, phenol & $\begin{array}{l}\mathrm{CH}_{\mathrm{n}}^{[\text {alc }}, \mathrm{CH}_{\mathrm{n}}^{[\text {alc-tail }]}, \mathrm{CH}_{\mathrm{n}}^{[\mathrm{OH}]}, \\
\mathrm{OH},\end{array}$ & $\begin{array}{l}\left(\mathrm{CH}_{3}^{[\text {alc }]}\right)\left(\mathrm{CH}_{3}^{\text {[alc-tail }]}\right) \\
\left(\mathrm{CH}_{2}^{\text {[alc-tail }]}\right)\left(\mathrm{CH}^{[\mathrm{OH}]}\right)(\mathrm{OH}),(\mathrm{ACH})_{5}(\mathrm{ACOH})\end{array}$ & 298 & LLE & 12 & 0.00 & De Oliveira and Aznar (2010) \\
\hline 2-butanol, phenol & $\begin{array}{l}\mathrm{CH}_{\mathrm{n}}^{[\text {alc }]}, \mathrm{CH}_{\mathrm{n}}^{[\text {alc-tail }]}, \mathrm{CH}_{\mathrm{n}}^{[\mathrm{OH}]}, \\
\mathrm{OH}, \\
\mathrm{ACH}_{\mathrm{n}}, \mathrm{ACOH}\end{array}$ & $\begin{array}{l}\left(\mathrm{CH}_{3}^{[\text {alc }]}\right)\left(\mathrm{CH}_{3}^{[\text {alc-tail }]}\right) \\
\left(\mathrm{CH}_{2}^{\text {[alc-tail] }}\right)\left(\mathrm{CH}^{[\mathrm{OH}]}\right)(\mathrm{OH}),(\mathrm{ACH})_{5}(\mathrm{ACOH})\end{array}$ & 313 & LLE & 12 & 1.00 & De Oliveira and Aznar (2010) \\
\hline \multicolumn{8}{|c|}{ - water + alcohol + aldehyde systems - } \\
\hline ethanol, butyraldehyde & $\begin{array}{l}\mathrm{CH}_{\mathrm{n}}, \mathrm{CH}_{\mathrm{n}}^{[\text {alc-tail }]}, \mathrm{CH}_{\mathrm{n}}^{[\mathrm{OH}]}, \mathrm{OH} \\
\mathrm{CHO}\end{array}$ & $\begin{array}{l}\left(\mathrm{CH}_{3}^{[\text {alc-tail }]}\right)\left(\mathrm{CH}_{2}^{[\mathrm{OH}]}\right)(\mathrm{OH}) \\
\left(\mathrm{CH}_{3}\right)\left(\mathrm{CH}_{2}\right)_{2}(\mathrm{CHO})\end{array}$ & 298 & LLE & 10 & 0.00 & Letcher et al. (1996) \\
\hline 2-propanol, butyraldehyde & $\begin{array}{l}\mathrm{CH}_{\mathrm{n}}, \mathrm{CH}_{\mathrm{n}}^{[\mathrm{alc}]}, \mathrm{CH}_{\mathrm{n}}^{[\mathrm{OH}]}, \mathrm{OH} \\
\mathrm{CHO}\end{array}$ & $\left(\mathrm{CH}_{3}^{[\mathrm{alc}]}\right)_{2}\left(\mathrm{CH}^{[\mathrm{OH}]}\right)(\mathrm{OH}),\left(\mathrm{CH}_{3}\right)\left(\mathrm{CH}_{2}\right)_{2}(\mathrm{CHO})$ & 298 & LLE & 10 & 0.00 & Letcher et al. (1996) \\
\hline 2-butanol, butyraldehyde & $\begin{array}{l}\mathrm{CH}_{\mathrm{n}}, \mathrm{CH}_{\mathrm{n}}^{[\text {alc-tail }]}, \\
\mathrm{CH}_{\mathrm{n}}^{[\mathrm{OH}]}, \mathrm{CH}, \mathrm{CHO}\end{array}$ & $\begin{array}{l}\left(\mathrm{CH}_{3}^{[\text {alc }]}\right)\left(\mathrm{CH}_{3}^{[\text {alc-tail }]}\right) \\
\left(\mathrm{CH}_{2}^{[\text {alc-tail }]}\right)\left(\mathrm{CH}^{[\mathrm{OH}]}\right)(\mathrm{OH}) \\
\left(\mathrm{CH}_{3}\right)\left(\mathrm{CH}_{2}\right)_{2}(\mathrm{CHO})\end{array}$ & 298 & LLE & 10 & 0.00 & Letcher et al. (1996) \\
\hline \multicolumn{8}{|c|}{ - water + acid + ketone systems -} \\
\hline acetic acid, 2-butanone & $\mathrm{CH}_{\mathrm{n}}, \mathrm{COOH}, \mathrm{CH}_{\mathrm{n}} \mathrm{CO}$ & $\left(\mathrm{CH}_{3}\right)(\mathrm{COOH}),\left(\mathrm{CH}_{3}\right)\left(\mathrm{CH}_{2}\right)\left(\mathrm{CH}_{3} \mathrm{CO}\right)$ & 298 & LLE & 8 & 0.00 & Correa et al. (1987) \\
\hline acetic acid, 2-butanone & $\mathrm{CH}_{\mathrm{n}}, \mathrm{COOH}, \mathrm{CH}_{\mathrm{n}} \mathrm{CO}$ & $\left(\mathrm{CH}_{3}\right)(\mathrm{COOH}),\left(\mathrm{CH}_{3}\right)\left(\mathrm{CH}_{2}\right)\left(\mathrm{CH}_{3} \mathrm{CO}\right)$ & 308 & LLE & 8 & 1.00 & Correa et al. (1987) \\
\hline acetic acid, 2-butanone & $\mathrm{CH}_{\mathrm{n}}, \mathrm{COOH}, \mathrm{CH}_{\mathrm{n}} \mathrm{CO}$ & $\left(\mathrm{CH}_{3}\right)(\mathrm{COOH}),\left(\mathrm{CH}_{3}\right)\left(\mathrm{CH}_{2}\right)\left(\mathrm{CH}_{3} \mathrm{CO}\right)$ & 318 & LLE & 8 & 1.00 & Correa et al. (1987) \\
\hline propanoic acid, 2-butanone & $\mathrm{CH}_{\mathrm{n}}, \mathrm{COOH}, \mathrm{CH}_{\mathrm{n}} \mathrm{CO}$ & $\left(\mathrm{CH}_{3}\right)\left(\mathrm{CH}_{2}\right)(\mathrm{COOH}),\left(\mathrm{CH}_{3}\right)\left(\mathrm{CH}_{2}\right)\left(\mathrm{CH}_{3} \mathrm{CO}\right)$ & 298 & LLE & 8 & 0.00 & Arce et al. (1995) \\
\hline propanoic acid, 2-butanone & $\mathrm{CH}_{\mathrm{n}}, \mathrm{COOH}, \mathrm{CH}_{\mathrm{n}} \mathrm{CO}$ & $\left(\mathrm{CH}_{3}\right)\left(\mathrm{CH}_{2}\right)(\mathrm{COOH}),\left(\mathrm{CH}_{3}\right)\left(\mathrm{CH}_{2}\right)\left(\mathrm{CH}_{3} \mathrm{CO}\right)$ & 308 & LLE & 12 & 1.00 & Arce et al. (1995) \\
\hline propanoic acid, 2-butanone & $\mathrm{CH}_{\mathrm{n}}, \mathrm{COOH}, \mathrm{CH}_{\mathrm{n}} \mathrm{CO}$ & $\left(\mathrm{CH}_{3}\right)\left(\mathrm{CH}_{2}\right)(\mathrm{COOH}),\left(\mathrm{CH}_{3}\right)\left(\mathrm{CH}_{2}\right)\left(\mathrm{CH}_{3} \mathrm{CO}\right)$ & 318 & LLE & 10 & 1.00 & Arce et al. (1995) \\
\hline $\begin{array}{l}\text { propanoic acid, 2- } \\
\text { pentanone }\end{array}$ & $\mathrm{CH}_{\mathrm{n}}, \mathrm{COOH}, \mathrm{CH}_{\mathrm{n}} \mathrm{CO}$ & $\left(\mathrm{CH}_{3}\right)\left(\mathrm{CH}_{2}\right)(\mathrm{COOH}),\left(\mathrm{CH}_{3}\right)\left(\mathrm{CH}_{2}\right)_{2}\left(\mathrm{CH}_{3} \mathrm{CO}\right)$ & 298 & LLE & 12 & 0.00 & Arce et al. (1995) \\
\hline $\begin{array}{l}\text { propanoic acid, } 2- \\
\text { pentanone }\end{array}$ & $\mathrm{CH}_{\mathrm{n}}, \mathrm{COOH}, \mathrm{CH}_{\mathrm{n}} \mathrm{CO}$ & $\left(\mathrm{CH}_{3}\right)\left(\mathrm{CH}_{2}\right)(\mathrm{COOH}),\left(\mathrm{CH}_{3}\right)\left(\mathrm{CH}_{2}\right)_{2}\left(\mathrm{CH}_{3} \mathrm{CO}\right)$ & 308 & LLE & 12 & 1.00 & Arce et al. (1995) \\
\hline $\begin{array}{l}\text { propanoic acid, } 2- \\
\text { pentanone }\end{array}$ & $\mathrm{CH}_{\mathrm{n}}, \mathrm{COOH}, \mathrm{CH}_{\mathrm{n}} \mathrm{CO}$ & $\left(\mathrm{CH}_{3}\right)\left(\mathrm{CH}_{2}\right)(\mathrm{COOH}),\left(\mathrm{CH}_{3}\right)\left(\mathrm{CH}_{2}\right)_{2}\left(\mathrm{CH}_{3} \mathrm{CO}\right)$ & 318 & LLE & 12 & 1.00 & Arce et al. (1995) \\
\hline $\begin{array}{l}\text { propanoic acid, 2- } \\
\text { pentanone }\end{array}$ & $\mathrm{CH}_{\mathrm{n}}, \mathrm{COOH}, \mathrm{CH}_{\mathrm{n}} \mathrm{CO}$ & $\left(\mathrm{CH}_{3}\right)\left(\mathrm{CH}_{2}\right)(\mathrm{COOH}),\left(\mathrm{CH}_{3}\right)\left(\mathrm{CH}_{2}\right)_{2}\left(\mathrm{CH}_{3} \mathrm{CO}\right)$ & 328 & LLE & 16 & 1.00 & Arce et al. (1995) \\
\hline \multicolumn{8}{|c|}{ - water + acid + ether systems - } \\
\hline $\begin{array}{l}\text { acetic acid, 2-methoxy-2- } \\
\text { methylpropane }\end{array}$ & $\mathrm{CH}_{\mathrm{n}}, \mathrm{COOH}, \mathrm{CH}_{\mathrm{n}} \mathrm{O}$ & $\left(\mathrm{CH}_{3}\right)(\mathrm{COOH}),\left(\mathrm{CH}_{3}\right)_{3}(\mathrm{C})\left(\mathrm{CH}_{3} \mathrm{O}\right)$ & 293 & LLE & 18 & 0.00 & Miao et al. (2007) \\
\hline $\begin{array}{l}\text { acetic acid, 2-methoxy-2- } \\
\text { methylpropane }\end{array}$ & $\mathrm{CH}_{\mathrm{n}}, \mathrm{COOH}, \mathrm{CH}_{\mathrm{n}} \mathrm{O}$ & $\left(\mathrm{CH}_{3}\right)(\mathrm{COOH}),\left(\mathrm{CH}_{3}\right)_{3}(\mathrm{C})\left(\mathrm{CH}_{3} \mathrm{O}\right)$ & 298 & LLE & 18 & 0.00 & Miao et al. (2007) \\
\hline $\begin{array}{l}\text { acetic acid, 2-methoxy-2- } \\
\text { methylpropane }\end{array}$ & $\mathrm{CH}_{\mathrm{n}}, \mathrm{COOH}, \mathrm{CH}_{\mathrm{n}} \mathrm{O}$ & $\left(\mathrm{CH}_{3}\right)(\mathrm{COOH}),\left(\mathrm{CH}_{3}\right)_{3}(\mathrm{C})\left(\mathrm{CH}_{3} \mathrm{O}\right)$ & 303 & LLE & 18 & 0.00 & Miao et al. (2007) \\
\hline $\begin{array}{l}\text { acetic acid, 2-methoxy-2- } \\
\text { methylpropane }\end{array}$ & $\mathrm{CH}_{\mathrm{n}}, \mathrm{COOH}, \mathrm{CH}_{\mathrm{n}} \mathrm{O}$ & $\left(\mathrm{CH}_{3}\right)(\mathrm{COOH}),\left(\mathrm{CH}_{3}\right)_{3}(\mathrm{C})\left(\mathrm{CH}_{3} \mathrm{O}\right)$ & 308 & LLE & 18 & 0.10 & Miao et al. (2007) \\
\hline \multicolumn{8}{|c|}{ - water + acid + ester systems -} \\
\hline acetic acid, ethyl acetate & $\mathrm{CH}_{\mathrm{n}}, \mathrm{COOH}, \mathrm{CCOO}$ & $\left(\mathrm{CH}_{3}\right)(\mathrm{COOH}),\left(\mathrm{CH}_{3}\right)\left(\mathrm{CH}_{2}\right)\left(\mathrm{CH}_{3} \mathrm{COO}\right)$ & 283 & LLE & 12 & 1.00 & Colombo et al. (1999) \\
\hline acetic acid, ethyl acetate & $\mathrm{CH}_{\mathrm{n}}, \mathrm{COOH}, \mathrm{CCOO}$ & $\left(\mathrm{CH}_{3}\right)(\mathrm{COOH}),\left(\mathrm{CH}_{3}\right)\left(\mathrm{CH}_{2}\right)\left(\mathrm{CH}_{3} \mathrm{COO}\right)$ & 298 & LLE & 12 & 0.00 & Colombo et al. (1999) \\
\hline acetic acid, ethyl acetate & $\mathrm{CH}_{\mathrm{n}}, \mathrm{COOH}, \mathrm{CCOO}$ & $\left(\mathrm{CH}_{3}\right)(\mathrm{COOH}),\left(\mathrm{CH}_{3}\right)\left(\mathrm{CH}_{2}\right)\left(\mathrm{CH}_{3} \mathrm{COO}\right)$ & 313 & LLE & 12 & 1.00 & Colombo et al. (1999) \\
\hline acetic acid, 1-butyl acetate & $\mathrm{CH}_{\mathrm{n}}, \mathrm{COOH}, \mathrm{CCOO}$ & $\left(\mathrm{CH}_{3}\right)(\mathrm{COOH}),\left(\mathrm{CH}_{3}\right)\left(\mathrm{CH}_{2}\right)_{3}\left(\mathrm{CH}_{3} \mathrm{COO}\right)$ & 304 & LLE & 18 & 0.00 & Wang et al. (2007) \\
\hline acetic acid, 1-butyl acetate & $\mathrm{CH}_{\mathrm{n}}, \mathrm{COOH}, \mathrm{CCOO}$ & $\left(\mathrm{CH}_{3}\right)(\mathrm{COOH}),\left(\mathrm{CH}_{3}\right)\left(\mathrm{CH}_{2}\right)_{3}\left(\mathrm{CH}_{3} \mathrm{COO}\right)$ & 332 & LLE & 16 & 1.00 & Wang et al. (2007) \\
\hline acetic acid, 1-butyl acetate & $\mathrm{CH}_{\mathrm{n}}, \mathrm{COOH}, \mathrm{CCOO}$ & $\left(\mathrm{CH}_{3}\right)(\mathrm{COOH}),\left(\mathrm{CH}_{3}\right)\left(\mathrm{CH}_{2}\right)_{3}\left(\mathrm{CH}_{3} \mathrm{COO}\right)$ & 366 & LLE & 16 & 1.00 & Wang et al. (2007) \\
\hline acetic acid, isobutyl acetate & $\mathrm{CH}_{\mathrm{n}}, \mathrm{COOH}, \mathrm{CCOO}$ & $\left(\mathrm{CH}_{3}\right)(\mathrm{COOH}),\left(\mathrm{CH}_{3}\right)_{2}\left(\mathrm{CH}_{2}\right)(\mathrm{CH})\left(\mathrm{CH}_{3} \mathrm{COO}\right)$ & 304 & LLE & 16 & 0.00 & Wang et al. (2007) \\
\hline acetic acid, isobutyl acetate & $\mathrm{CH}_{\mathrm{n}}, \mathrm{COOH}, \mathrm{CCOO}$ & $\left(\mathrm{CH}_{3}\right)(\mathrm{COOH}),\left(\mathrm{CH}_{3}\right)_{2}\left(\mathrm{CH}_{2}\right)(\mathrm{CH})\left(\mathrm{CH}_{3} \mathrm{COO}\right)$ & 332 & LLE & 16 & 1.00 & Wang et al. (2007) \\
\hline acetic acid, isobutyl acetate & $\mathrm{CH}_{\mathrm{n}}, \mathrm{COOH}, \mathrm{CCOO}$ & $\left(\mathrm{CH}_{3}\right)(\mathrm{COOH}),\left(\mathrm{CH}_{3}\right)_{2}\left(\mathrm{CH}_{2}\right)(\mathrm{CH})\left(\mathrm{CH}_{3} \mathrm{COO}\right)$ & 366 & LLE & 14 & 1.00 & Wang et al. (2007) \\
\hline \multicolumn{8}{|c|}{- water + acid + aromatic systems -} \\
\hline acetic acid, benzene & $\mathrm{CH}_{\mathrm{n}}, \mathrm{COOH}, \mathrm{ACH}_{\mathrm{n}}$ & $\left(\mathrm{CH}_{3}\right)(\mathrm{COOH}),(\mathrm{ACH})_{6}$ & 298 & LLE & 10 & 0.00 & Backes et al. (1990) \\
\hline \multicolumn{8}{|c|}{ - water + ketone + ether systems - } \\
\hline $\begin{array}{ll}\text { 2-butanone, } & 2- \\
\text { butoxyethanol } & \end{array}$ & $\begin{array}{l}\mathrm{CH}_{\mathrm{n}}, \mathrm{CH}_{\mathrm{n}}^{[\mathrm{OH}]}, \mathrm{CH}_{\mathrm{n}} \mathrm{O}, \mathrm{OH} \\
\mathrm{CH}_{\mathrm{n}} \mathrm{CO}\end{array}$ & $\begin{array}{l}\left(\mathrm{CH}_{3}\right)\left(\mathrm{CH}_{2}\right)\left(\mathrm{CH}_{3} \mathrm{CO}\right) \\
\left(\mathrm{CH}_{3}\right)\left(\mathrm{CH}_{2}\right)_{3}\left(\mathrm{CH}_{2}^{[\mathrm{OH}]}\right)\left(\mathrm{CH}_{2} \mathrm{O}\right)(\mathrm{OH})\end{array}$ & 298 & LLE & 10 & 0.00 & Newman et al. (1949) \\
\hline
\end{tabular}


Table 1. Continued.

\begin{tabular}{|c|c|c|c|c|c|c|c|}
\hline Organic compounds & Org. main groups & Chemical formula (subgroups) & $T(\mathrm{~K})$ & Data type & $N_{d}$ & $w_{d}^{\text {init }}$ & Reference \\
\hline \multicolumn{8}{|c|}{ - water + ketone + ester systems -} \\
\hline \multicolumn{8}{|c|}{ - water + ketone + aromatic systems -} \\
\hline acetone, phenol & $\begin{array}{l}\mathrm{CH}_{\mathrm{n}}, \mathrm{CH}_{\mathrm{n}} \mathrm{CO}, \mathrm{ACH}_{\mathrm{n}} \\
\mathrm{ACOH}\end{array}$ & $\left(\mathrm{CH}_{3}\right)\left(\mathrm{CH}_{3} \mathrm{CO}\right),(\mathrm{ACH})_{5}(\mathrm{ACOH})$ & 323 & LLE & 24 & 1.00 & Mafra and Krähenbühl (2006) \\
\hline acetone, phenol & $\begin{array}{l}\mathrm{CH}_{\mathrm{n}}, \mathrm{CH}_{\mathrm{n}} \mathrm{CO}, \mathrm{ACH}_{\mathrm{n}} \\
\mathrm{ACOH}\end{array}$ & $\left(\mathrm{CH}_{3}\right)\left(\mathrm{CH}_{3} \mathrm{CO}\right),(\mathrm{ACH})_{5}(\mathrm{ACOH})$ & 333 & LLE & 22 & 1.00 & Mafra and Krähenbühl (2006) \\
\hline \multicolumn{8}{|c|}{ - water + ether + aromatic systems - } \\
\hline $\begin{array}{l}\text { 2-methoxy-2- } \\
\text { methylpropane, benzene }\end{array}$ & $\mathrm{CH}_{\mathrm{n}}, \mathrm{CH}_{\mathrm{n}} \mathrm{O}, \mathrm{ACH}_{\mathrm{n}}$ & $\left(\mathrm{CH}_{3}\right)_{3}(\mathrm{C})\left(\mathrm{CH}_{3} \mathrm{O}\right),(\mathrm{ACH})_{6}$ & 298 & LLE & 30 & 0.00 & Stephenson (1992) \\
\hline \multicolumn{8}{|c|}{ - water + ether + aldehyde systems - } \\
\hline diethyl ether, acetaldehyde & $\mathrm{CH}_{\mathrm{n}}, \mathrm{CH}_{\mathrm{n}} \mathrm{O}, \mathrm{CHO}$ & $\left(\mathrm{CH}_{3}\right)_{2}\left(\mathrm{CH}_{2}\right) \mathrm{CH}_{2} \mathrm{O},\left(\mathrm{CH}_{3}\right)(\mathrm{CHO})$ & 288 & LLE & 10 & 0.20 & Suska (1979) \\
\hline \multicolumn{8}{|c|}{ - water + ester + aromatic systems - } \\
\hline ethyl acetate, phenol & $\mathrm{CH}_{\mathrm{n}}, \mathrm{CCOO}, \mathrm{ACH}_{\mathrm{n}}, \mathrm{ACOH}$ & $\left(\mathrm{CH}_{3}\right)\left(\mathrm{CH}_{2}\right)\left(\mathrm{CH}_{3} \mathrm{COO}\right),(\mathrm{ACH})_{5}(\mathrm{ACOH})$ & 298 & LLE & 18 & 0.00 & Alvarez Gonzalez et al. (1986) \\
\hline 1-butyl acetate, phenol & $\mathrm{CH}_{\mathrm{n}}, \mathrm{CCOO}, \mathrm{ACH}_{\mathrm{n}}, \mathrm{ACOH}$ & $\left(\mathrm{CH}_{3}\right)\left(\mathrm{CH}_{2}\right)_{3}\left(\mathrm{CH}_{3} \mathrm{COO}\right),(\mathrm{ACH})_{5}(\mathrm{ACOH})$ & 298 & LLE & 32 & 0.00 & Takahashi et al. (1988) \\
\hline 1-butyl acetate, phenol & $\mathrm{CH}_{\mathrm{n}}, \mathrm{CCOO}, \mathrm{ACH}_{\mathrm{n}}, \mathrm{ACOH}$ & $\left(\mathrm{CH}_{3}\right)\left(\mathrm{CH}_{2}\right)_{3}\left(\mathrm{CH}_{3} \mathrm{COO}\right),(\mathrm{ACH})_{5}(\mathrm{ACOH})$ & 313 & LLE & 32 & 0.50 & Takahashi et al. (1988) \\
\hline \multicolumn{8}{|l|}{ - water-free systems - } \\
\hline ethanol, acetic acid & $\begin{array}{l}\mathrm{CH}_{\mathrm{n}}, \mathrm{CH}_{\mathrm{n}}^{[\text {alc-tail }]}, \mathrm{CH}_{\mathrm{n}}^{[\mathrm{OH}]}, \mathrm{OH}, \\
\mathrm{COOH}\end{array}$ & $\left(\mathrm{CH}_{3}^{[\text {alc-tail }]}\right)\left(\mathrm{CH}_{2}^{[\mathrm{OH}]}\right)(\mathrm{OH}),\left(\mathrm{CH}_{3}\right)(\mathrm{COOH})$ & $244-284$ & SLE(org) ${ }^{\mathrm{d}}$ & 13 & 0.20 & Carta and Dernini (1983) \\
\hline ethanol, acetic acid & $\begin{array}{l}\mathrm{CH}_{\mathrm{n}}, \mathrm{CH}_{\mathrm{n}}^{[\text {alc-tail }]}, \mathrm{CH}_{\mathrm{n}}^{[\mathrm{OH}]}, \mathrm{OH}, \\
\mathrm{COOH}\end{array}$ & $\left(\mathrm{CH}_{3}^{[\text {alc-tail }]}\right)\left(\mathrm{CH}_{2}^{[\mathrm{OH}]}\right)(\mathrm{OH}),\left(\mathrm{CH}_{3}\right)(\mathrm{COOH})$ & $241-289$ & $\operatorname{SLE}(\text { org })^{\mathrm{d}}$ & 22 & 0.20 & Pickering (1893) \\
\hline ethanol, acetic acid & $\begin{array}{l}\mathrm{CH}_{\mathrm{n}}, \mathrm{CH}_{\mathrm{n}}^{[\text {alc-tail }]}, \mathrm{CH}_{\mathrm{n}}^{[\mathrm{OH}]}, \mathrm{OH} \\
\mathrm{COOH}\end{array}$ & $\left(\mathrm{CH}_{3}^{[\text {alc-tail }]}\right)\left(\mathrm{CH}_{2}^{[\mathrm{OH}]}\right)(\mathrm{OH}),\left(\mathrm{CH}_{3}\right)(\mathrm{COOH})$ & $354-389$ & VLE & 12 & 0.10 & Reichl et al. (1998) \\
\hline ethanol, acetic acid & $\begin{array}{l}\mathrm{CH}_{\mathrm{n}}, \mathrm{CH}_{\mathrm{n}}^{[\text {alc-tail }]}, \mathrm{CH}_{\mathrm{n}}^{[\mathrm{OH}]}, \mathrm{OH}, \\
\mathrm{COOH}\end{array}$ & $\left(\mathrm{CH}_{3}^{[\text {alc-tail }]}\right)\left(\mathrm{CH}_{2}^{[\mathrm{OH}]}\right)(\mathrm{OH}),\left(\mathrm{CH}_{3}\right)(\mathrm{COOH})$ & $351-386$ & VLE & 16 & 0.10 & Hirata et al. (1975) \\
\hline ethanol, acetic acid & $\begin{array}{l}\mathrm{CH}_{\mathrm{n}}, \mathrm{CH}_{\mathrm{n}}^{[\text {alc-tail }]}, \mathrm{CH}_{\mathrm{n}}^{[\mathrm{OH}]}, \mathrm{OH}, \\
\mathrm{COOH}\end{array}$ & $\left(\mathrm{CH}_{3}^{[\text {alc-tail }]}\right)\left(\mathrm{CH}_{2}^{[\mathrm{OH}]}\right)(\mathrm{OH}),\left(\mathrm{CH}_{3}\right)(\mathrm{COOH})$ & 323 & VLE & 16 & 0.10 & Miyamoto et al. (2001) \\
\hline 1-propanol, acetic acid & $\begin{array}{l}\mathrm{CH}_{\mathrm{n}}, \mathrm{CH}_{\mathrm{n}}^{[\text {alc-tail }]}, \mathrm{CH}_{\mathrm{n}}^{[\mathrm{OH}]}, \mathrm{OH}, \\
\mathrm{COOH}\end{array}$ & $\begin{array}{l}\left(\mathrm{CH}_{3}^{\text {[alc-tail }]}\right)\left(\mathrm{CH}_{2}^{[\text {alc-tail }]}\right) \\
\left(\mathrm{CH}_{3}\right)(\mathrm{COOH})\end{array}$ & $254-287$ & SLE(org) $)^{d}$ & 13 & 0.20 & Pickering (1893) \\
\hline 1-propanol, acetic acid & $\begin{array}{l}\mathrm{CH}_{\mathrm{n}}, \mathrm{CH}_{\mathrm{n}}^{[\text {alc-tail }}, \mathrm{CH}_{\mathrm{n}}^{[\mathrm{OH}]}, \mathrm{OH}, \\
\mathrm{COOH}\end{array}$ & $\begin{array}{l}\left(\mathrm{CH}_{3}^{\text {[alc-tail }]}\right)\left(\mathrm{CH}_{2}^{[\text {[alc-tail }]}\right) \quad\left(\mathrm{CH}_{2}^{[\mathrm{OH}]}\right)(\mathrm{OH}), \\
\left(\mathrm{CH}_{3}\right)(\mathrm{COOH})\end{array}$ & $370-387$ & VLE & 14 & 1.00 & Rius et al. (1959) \\
\hline cyclohexanol, adipic acid & $\begin{array}{l}\mathrm{CH}_{\mathrm{n}}, \mathrm{CH}_{\mathrm{n}}^{[\text {alc-tail }]}, \mathrm{CH}_{\mathrm{n}}^{[\mathrm{OH}]}, \mathrm{OH} \\
\mathrm{COOH}\end{array}$ & $\left(\mathrm{CH}_{2}^{[\text {alc-tail }]}\right)_{5}\left(\mathrm{CH}^{[\mathrm{OH}]}\right)(\mathrm{OH}),\left(\mathrm{CH}_{2}\right)_{4}(\mathrm{COOH})_{2}$ & $299-352$ & SLE(org) $)^{d}$ & 12 & 0.10 & Lihua et al. (2007) \\
\hline ethanol, acetone & $\begin{array}{l}\mathrm{CH}_{n}, \mathrm{CH}_{n}^{[\text {alc-tail }]}, \mathrm{CH}_{\mathrm{n}}^{[\mathrm{OH}]}, \mathrm{OH}, \\
\mathrm{CH}_{\mathrm{n}} \mathrm{CO}\end{array}$ & $\left(\mathrm{CH}_{3}^{[\text {alc-tail }]}\right)\left(\mathrm{CH}_{2}^{[\mathrm{OH}]}\right)(\mathrm{OH}),\left(\mathrm{CH}_{3}\right)\left(\mathrm{CH}_{3} \mathrm{CO}\right)$ & $154-173$ & SLE & 6 & 0.20 & Sapgir (1929) \\
\hline ethanol, acetone & $\begin{array}{l}\mathrm{CH}_{n}, \mathrm{CH}_{\mathrm{n}}^{[\text {alc-tail }]}, \mathrm{CH}_{\mathrm{n}}^{[\mathrm{OH}]}, \mathrm{OH}, \\
\mathrm{CH}_{\mathrm{n}} \mathrm{CO}\end{array}$ & $\left(\mathrm{CH}_{3}^{[\text {alc-tail }]}\right)\left(\mathrm{CH}_{2}^{[\mathrm{OH}]}\right)(\mathrm{OH}),\left(\mathrm{CH}_{3}\right)\left(\mathrm{CH}_{3} \mathrm{CO}\right)$ & 344 & VLE & 9 & 1.00 & Lee and Hu (1995) \\
\hline ethanol, acetone & $\begin{array}{l}\mathrm{CH}_{\mathrm{n}}, \mathrm{CH}_{\mathrm{n}}^{[\text {alc-tail }]}, \mathrm{CH}_{\mathrm{n}}^{[\mathrm{OH}]}, \mathrm{OH}, \\
\mathrm{CH}_{\mathrm{n}} \mathrm{CO}\end{array}$ & $\left(\mathrm{CH}_{3}^{[\text {alc-tail }]}\right)\left(\mathrm{CH}_{2}^{[\mathrm{OH}]}\right)(\mathrm{OH}),\left(\mathrm{CH}_{3}\right)\left(\mathrm{CH}_{3} \mathrm{CO}\right)$ & 353 & VLE & 9 & 1.00 & Lee and Hu (1995) \\
\hline ethanol, acetone & $\begin{array}{l}\mathrm{CH}_{n}, \mathrm{CH}_{n}^{[\text {alc-tail }]}, \mathrm{CH}_{\mathrm{n}}^{[\mathrm{OH}]}, \mathrm{OH}, \\
\mathrm{CH}_{\mathrm{n}} \mathrm{CO}\end{array}$ & $\left(\mathrm{CH}_{3}^{[\text {alc-tail }]}\right)\left(\mathrm{CH}_{2}^{[\mathrm{OH}]}\right)(\mathrm{OH}),\left(\mathrm{CH}_{3}\right)\left(\mathrm{CH}_{3} \mathrm{CO}\right)$ & 363 & VLE & 9 & 1.00 & Lee and $\mathrm{Hu}$ (1995) \\
\hline ethanol, acetone & $\begin{array}{l}\mathrm{CH}_{\mathrm{n}}, \mathrm{CH}_{\mathrm{n}}^{[\text {alc-tail }]}, \mathrm{CH}_{\mathrm{n}}^{[\mathrm{OH}]}, \mathrm{OH}, \\
\mathrm{CH}_{\mathrm{n}} \mathrm{CO}\end{array}$ & $\left(\mathrm{CH}_{3}^{[\text {alc-tail }]}\right)\left(\mathrm{CH}_{2}^{[\mathrm{OH}]}\right)(\mathrm{OH}),\left(\mathrm{CH}_{3}\right)\left(\mathrm{CH}_{3} \mathrm{CO}\right)$ & 373 & VLE & 9 & 1.00 & Campbell et al. (1987) \\
\hline ethanol, acetone & $\begin{array}{l}\mathrm{CH}_{\mathrm{n}}, \mathrm{CH}_{\mathrm{n}}^{[\text {alc-tail }]}, \mathrm{CH}_{\mathrm{n}}^{[\mathrm{OH}]}, \mathrm{OH}, \\
\mathrm{CH}_{\mathrm{n}} \mathrm{CO}\end{array}$ & $\left(\mathrm{CH}_{3}^{[\text {alc-tail }]}\right)\left(\mathrm{CH}_{2}^{[\mathrm{OH}]}\right)(\mathrm{OH}),\left(\mathrm{CH}_{3}\right)\left(\mathrm{CH}_{3} \mathrm{CO}\right)$ & 398 & VLE & 11 & 1.00 & Campbell et al. (1987) \\
\hline ethanol, acetone & $\begin{array}{l}\mathrm{CH}_{\mathrm{n}}, \mathrm{CH}_{\mathrm{n}}^{[\text {alc-tail }]}, \mathrm{CH}_{\mathrm{n}}^{[\mathrm{OH}]}, \mathrm{OH} \\
\mathrm{CH}_{\mathrm{n}} \mathrm{CO}\end{array}$ & $\left(\mathrm{CH}_{3}^{[\text {alc-tail }]}\right)\left(\mathrm{CH}_{2}^{[\mathrm{OH}]}\right)(\mathrm{OH}),\left(\mathrm{CH}_{3}\right)\left(\mathrm{CH}_{3} \mathrm{CO}\right)$ & 423 & VLE & 10 & 1.00 & Campbell et al. (1987) \\
\hline ethanol, acetone & $\begin{array}{l}\mathrm{CH}_{\mathrm{n}}, \mathrm{CH}_{\mathrm{n}}^{[\text {alc-tail }]}, \mathrm{CH}_{\mathrm{n}}^{[\mathrm{OH}]}, \mathrm{OH}, \\
\mathrm{CH}_{\mathrm{n}} \mathrm{CO}\end{array}$ & $\left(\mathrm{CH}_{3}^{[\text {alc-tail }]}\right)\left(\mathrm{CH}_{2}^{[\mathrm{OH}]}\right)(\mathrm{OH}),\left(\mathrm{CH}_{3}\right)\left(\mathrm{CH}_{3} \mathrm{CO}\right)$ & $330-350$ & VLE & 9 & 1.00 & Amer et al. (1956) \\
\hline ethanol, 2-butanone & 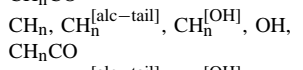 & $\begin{array}{l}\left(\mathrm{CH}_{3}^{\text {[alc-tail }]}\right)\left(\mathrm{CH}_{2}^{[\mathrm{OH}]}\right)(\mathrm{OH}) \\
\left(\mathrm{CH}_{3}\right)\left(\mathrm{CH}_{2}\right)\left(\mathrm{CH}_{3} \mathrm{CO}\right)\end{array}$ & 298 & VLE & 12 & 0.00 & Ohta et al. (1981) \\
\hline ethanol, 2-butanone & $\begin{array}{l}\mathrm{CH}_{\mathrm{n}}, \mathrm{CH}_{\mathrm{n}}^{[\text {alc-tail }]}, \mathrm{CH}_{\mathrm{n}}^{[\mathrm{OH}]}, \mathrm{OH} \\
\mathrm{CH}_{\mathrm{n}} \mathrm{CO}\end{array}$ & $\begin{array}{l}\left(\mathrm{CH}_{3}^{[\text {alc-tail] }}\right)\left(\mathrm{CH}_{2}^{[\mathrm{OH}]}\right)(\mathrm{OH}) \\
\left(\mathrm{CH}_{3}\right)\left(\mathrm{CH}_{2}\right)\left(\mathrm{CH}_{3} \mathrm{CO}\right)\end{array}$ & $308-314$ & VLE & 19 & 1.00 & Martínez et al. (2008) \\
\hline ethanol, 2-butanone & $\begin{array}{l}\mathrm{CH}_{\mathrm{n}}, \mathrm{CH}_{\mathrm{n}}^{[\text {alc-tail }]}, \mathrm{CH}_{\mathrm{n}}^{[\mathrm{OH}]}, \mathrm{OH}, \\
\mathrm{CH}_{\mathrm{n}} \mathrm{CO}\end{array}$ & 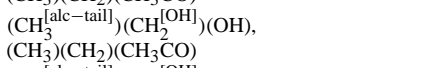 & $348-351$ & VLE & 19 & 1.00 & Martínez et al. (2008) \\
\hline ethanol, 2-butanone & $\begin{array}{l}\mathrm{CH}_{\mathrm{n}}, \mathrm{CH}_{\mathrm{n}}^{[\text {alc-tail }]}, \mathrm{CH}_{\mathrm{n}}^{[\mathrm{OH}]}, \mathrm{OH} \\
\mathrm{CH}_{\mathrm{n}} \mathrm{CO}\end{array}$ & $\begin{array}{l}\left(\mathrm{CH}_{3}^{\text {alc }- \text { tail }]}\right)\left(\mathrm{CH}_{2}^{[\mathrm{OH}]}\right)(\mathrm{OH}) \\
\left(\mathrm{CH}_{3}\right)\left(\mathrm{CH}_{2}\right)\left(\mathrm{CH}_{3} \mathrm{CO}\right)\end{array}$ & $347-352$ & VLE & 19 & 1.00 & Wen and Tu (2007) \\
\hline ethanol, 2-heptanone & $\begin{array}{l}\mathrm{CH}_{\mathrm{n}}, \mathrm{CH}_{\mathrm{n}}^{[\text {alc-tail }]}, \mathrm{CH}_{\mathrm{n}}^{[\mathrm{OH}]}, \mathrm{OH} \\
\mathrm{CH}_{\mathrm{n}} \mathrm{CO}\end{array}$ & $\begin{array}{l}\left(\mathrm{CH}_{3}^{[\text {alc-tail }]}\right)\left(\mathrm{CH}_{2}^{[\mathrm{OH}]}\right)(\mathrm{OH}) \\
\left(\mathrm{CH}_{3}\right)\left(\mathrm{CH}_{2}\right)_{4}\left(\mathrm{CH}_{3} \mathrm{CO}\right)\end{array}$ & $208-238$ & SLE(org) ${ }^{d}$ & 20 & 0.20 & Fiege et al. (1996) \\
\hline ethanol, 3-heptanone & $\begin{array}{l}\mathrm{CH}_{\mathrm{n}}, \mathrm{CH}_{\mathrm{n}}^{[\text {alc-tail }]}, \mathrm{CH}_{\mathrm{n}}^{[\mathrm{OH}]}, \mathrm{OH}, \\
\mathrm{CH}_{\mathrm{n}} \mathrm{CO}\end{array}$ & $\begin{array}{l}\left(\mathrm{CH}_{3}^{[\text {alc-tail }]}\right)\left(\mathrm{CH}_{2}^{[\mathrm{OH}]}\right)(\mathrm{OH}) \\
\left(\mathrm{CH}_{3}\right)_{2}\left(\mathrm{CH}_{2}\right)_{3}\left(\mathrm{CH}_{2} \mathrm{CO}\right)\end{array}$ & $204-236$ & SLE(org) ${ }^{\mathrm{d}}$ & 20 & 0.20 & Fiege et al. (1996) \\
\hline ethanol, 4-heptanone & $\begin{array}{l}\mathrm{CH}_{n}, \mathrm{CH}_{\mathrm{n}}^{[\text {alc-tail }]}, \mathrm{CH}_{\mathrm{n}}^{[\mathrm{OH}]}, \mathrm{OH}, \\
\mathrm{CH}_{\mathrm{n}} \mathrm{CO}\end{array}$ & $\begin{array}{l}\left(\mathrm{CH}_{3}^{[\text {alc-tail }]}\right)\left(\mathrm{CH}_{2}^{[\mathrm{OH}]}\right)(\mathrm{OH}) \\
\left(\mathrm{CH}_{3}\right)_{2}\left(\mathrm{CH}_{2}\right)_{3}\left(\mathrm{CH}_{3} \mathrm{CO}\right)\end{array}$ & $205-240$ & SLE(org) $)^{d}$ & 20 & 0.20 & Fiege et al. (1996) \\
\hline 1-hexanol, 2-octanone & $\begin{array}{l}\mathrm{CH}_{n}, \mathrm{CH}_{\mathrm{n}}^{[\text {alc-tail }]}, \mathrm{CH}_{\mathrm{n}}^{[\mathrm{OH}]}, \mathrm{OH} \\
\mathrm{CH}_{\mathrm{n}} \mathrm{CO}\end{array}$ & $\begin{array}{l}\left(\mathrm{CH}_{3}^{\text {[alc-tail }]}\right)\left(\mathrm{CH}_{2}^{[\text {alc-tail }]}\right)_{4}\left(\mathrm{CH}_{2}^{[\mathrm{OH}]}\right)(\mathrm{OH}), \\
\left(\mathrm{CH}_{3}\right)\left(\mathrm{CH}_{2}\right){ }_{5}\left(\mathrm{CH}_{3} \mathrm{CO}\right)\end{array}$ & $227-253$ & SLE(org) $)^{\mathrm{d}}$ & 20 & 0.20 & Abbas and Gmehling (2008) \\
\hline ethanol, diethyl ether & $\begin{array}{l}\mathrm{CH}_{n}, \mathrm{CH}_{\mathrm{n}}^{[\text {alc-tail }]}, \mathrm{CH}_{\mathrm{n}}^{[\mathrm{OH}]}, \mathrm{OH}, \\
\mathrm{CH}_{\mathrm{n}} \mathrm{O}\end{array}$ & $\begin{array}{l}\left(\mathrm{CH}_{3}^{[\text {[alc-tail }]}\right)\left(\mathrm{CH}_{2}^{[\mathrm{OH}]}\right)(\mathrm{OH}) \\
\left(\mathrm{CH}_{3}\right)_{2}\left(\mathrm{CH}_{2}\right) \mathrm{CH}_{2} \mathrm{O}\end{array}$ & $151-157$ & SLE(org) $)^{d}$ & 4 & 0.20 & Sapgir (1929) \\
\hline ethanol, diethyl ether & $\begin{array}{l}\mathrm{CH}_{\mathrm{n}}, \mathrm{CH}_{\mathrm{n}}^{[\text {alc-tail }]}, \mathrm{CH}_{\mathrm{n}}^{[\mathrm{OH}]}, \mathrm{OH}, \\
\mathrm{CH}_{\mathrm{n}} \mathrm{O}\end{array}$ & $\begin{array}{l}\left(\mathrm{CH}_{3}^{\text {[alc-tail] }}\right)\left(\mathrm{CH}_{2}^{[\mathrm{OH}]}\right)(\mathrm{OH}) \\
\left(\mathrm{CH}_{3}\right)_{2}\left(\mathrm{CH}_{2}\right) \mathrm{CH}_{2} \mathrm{O}\end{array}$ & $149-157$ & SLE(org) ${ }^{\mathrm{d}}$ & 13 & 0.20 & Lalande (1934) \\
\hline ethanol, diethyl ether & $\begin{array}{l}\mathrm{CH}_{\mathrm{n}}, \mathrm{CH}_{\mathrm{n}}^{[\text {alc-tail }]}, \mathrm{CH}_{\mathrm{n}}^{[\mathrm{OH}]}, \mathrm{OH}, \\
\mathrm{CH}_{\mathrm{n}} \mathrm{O}\end{array}$ & $\begin{array}{l}\left(\mathrm{CH}_{3}^{\text {[alc-tail] }}\right)\left(\mathrm{CH}_{2}^{[\mathrm{OH}]}\right)(\mathrm{OH}) \\
\left(\mathrm{CH}_{3}\right)_{2}\left(\mathrm{CH}_{2}\right) \mathrm{CH}_{2} \mathrm{O}\end{array}$ & $154-159$ & SLE(org) $)^{\mathrm{d}}$ & 2 & 0.20 & Sapgir (1929) \\
\hline
\end{tabular}


Table 1. Continued.

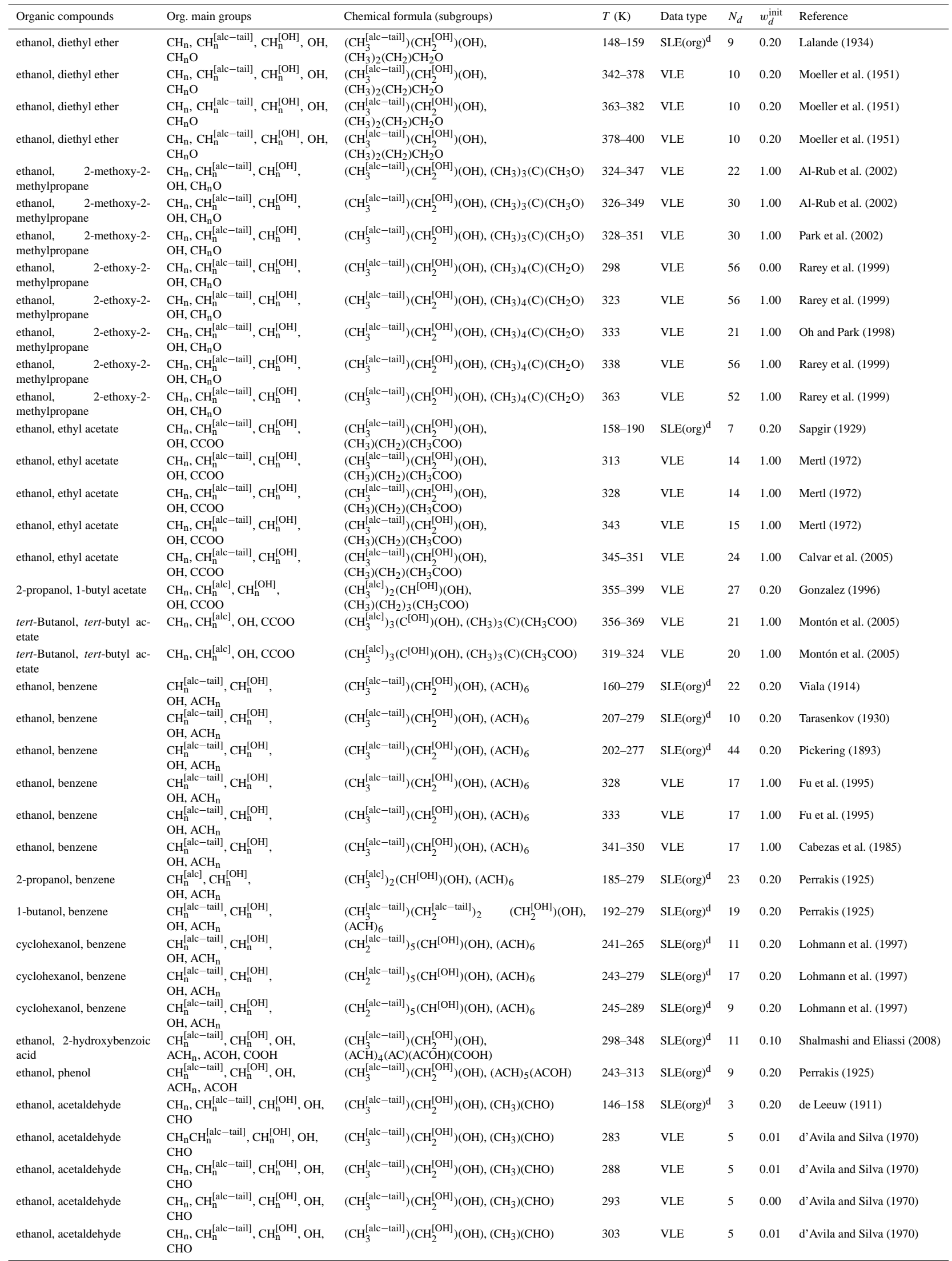


Table 1. Continued.

\begin{tabular}{|c|c|c|c|c|c|c|c|}
\hline Organic compounds & Org. main groups & Chemical formula (subgroups) & $T(\mathrm{~K})$ & Data type & $N_{d}$ & $w_{d}^{\text {init }}$ & Reference \\
\hline ethanol, acetaldehyde & $\begin{array}{l}\mathrm{CH}_{\mathrm{n}}, \mathrm{CH}_{\mathrm{n}}^{[\text {alc-tail }]}, \mathrm{CH}_{\mathrm{n}}^{[\mathrm{OH}]}, \mathrm{OH}, \\
\mathrm{CHO}\end{array}$ & $\left(\mathrm{CH}_{3}^{[\text {alc-tail }]}\right)\left(\mathrm{CH}_{2}^{[\mathrm{OH}]}\right)(\mathrm{OH}),\left(\mathrm{CH}_{3}\right)(\mathrm{CHO})$ & $302-350$ & VLE & 5 & 0.01 & Suska (1979) \\
\hline ethanol, butyraldehyde & $\begin{array}{l}\mathrm{CH}_{\mathrm{n}}, \mathrm{CH}_{\mathrm{n}}^{[\text {[alc-tail] }}, \mathrm{CH}_{\mathrm{n}}^{[\mathrm{OH}]}, \mathrm{OH}, \\
\mathrm{CHO}\end{array}$ & $\begin{array}{l}\left(\mathrm{CH}_{3}^{[\text {alc-tail }]}\right)\left(\mathrm{CH}_{2}^{[\mathrm{OH}]}\right)(\mathrm{OH}), \\
\left(\mathrm{CH}_{3}\right)\left(\mathrm{CH}_{2}\right)_{2}(\mathrm{CHO})\end{array}$ & 323 & VLE & 9 & 1.0 & Gmehling et al. (1988) \\
\hline ethanol, butyraldehyde & $\begin{array}{l}\mathrm{CH}_{\mathrm{n}}, \mathrm{CH}_{\mathrm{n}}^{[\text {[alc-tail }]}, \mathrm{CH}_{\mathrm{n}}^{[\mathrm{OH}]}, \mathrm{OH}, \\
\mathrm{CHO}\end{array}$ & $\begin{array}{l}\left(\mathrm{CH}_{3}^{\text {alc-tail }]}\right)\left(\mathrm{CH}_{2}^{[\mathrm{OH}]}\right)(\mathrm{OH}), \\
\left(\mathrm{CH}_{3}\right)\left(\mathrm{CH}_{2}\right)_{2}(\mathrm{CHO})\end{array}$ & 333 & VLE & 9 & 1.0 & Gmehling et al. (1988) \\
\hline ethanol, butyraldehyde & $\begin{array}{l}\mathrm{CH}_{\mathrm{n}}, \mathrm{CH}_{\mathrm{n}}^{[\text {[alc-tail] }}, \mathrm{CH}_{\mathrm{n}}^{[\mathrm{OH}]}, \mathrm{OH}, \\
\mathrm{CHO}\end{array}$ & $\begin{array}{l}\left(\mathrm{CH}_{3}^{[\text {[alc-tail }]}\right)\left(\mathrm{CH}_{2}^{[\mathrm{OH}]}\right)(\mathrm{OH}), \\
\left(\mathrm{CH}_{3}\right)\left(\mathrm{CH}_{2}\right)_{2}(\mathrm{CHO})\end{array}$ & 343 & VLE & 9 & 1.0 & Gmehling et al. (1988) \\
\hline ethanol, butyraldehyde & $\begin{array}{l}\mathrm{CH}_{\mathrm{n}}, \mathrm{CH}_{\mathrm{n}}^{[\text {alc-tail }]}, \mathrm{CH}_{\mathrm{n}}^{[\mathrm{OH}]}, \mathrm{OH}, \\
\mathrm{CHO}\end{array}$ & $\begin{array}{l}\left(\mathrm{CH}_{3}^{\text {[alc-tail }]}\right)\left(\mathrm{CH}_{2}^{[\mathrm{OH}]}\right)(\mathrm{OH}), \\
\left(\mathrm{CH}_{3}\right)\left(\mathrm{CH}_{2}\right)_{2}(\mathrm{CHO})\end{array}$ & 353 & VLE & 9 & 1.0 & Gmehling et al. (1988) \\
\hline ethanol, butyraldehyde & $\begin{array}{l}\mathrm{CH}_{\mathrm{n}}, \mathrm{CH}_{\mathrm{n}}^{[\text {[alc-tail] }}, \mathrm{CH}_{\mathrm{n}}^{[\mathrm{OH}]}, \mathrm{OH}, \\
\mathrm{CHO}\end{array}$ & $\begin{array}{l}\left(\mathrm{CH}_{3}^{\text {alc-tail }]}\right)\left(\mathrm{CH}_{2}^{[\mathrm{OH}]}\right)(\mathrm{OH}), \\
\left(\mathrm{CH}_{3}\right)\left(\mathrm{CH}_{2}\right)_{2}(\mathrm{CHO})\end{array}$ & $346-350$ & VLE & 15 & 1.0 & Gmehling et al. (1988) \\
\hline acetic acid, acetone & $\mathrm{CH}_{\mathrm{n}}, \mathrm{COOH}, \mathrm{CH}_{\mathrm{n}} \mathrm{CO}$ & $\left(\mathrm{CH}_{3}\right)(\mathrm{COOH}),\left(\mathrm{CH}_{3}\right)\left(\mathrm{CH}_{3} \mathrm{CO}\right)$ & 173 & SLE(org) ${ }^{\mathrm{d}}$ & 1 & 0.20 & Chesnokov (1969) \\
\hline acetic acid, acetone & $\mathrm{CH}_{\mathrm{n}}, \mathrm{COOH}, \mathrm{CH}_{\mathrm{n}} \mathrm{CO}$ & $\left(\mathrm{CH}_{3}\right)(\mathrm{COOH}),\left(\mathrm{CH}_{3}\right)\left(\mathrm{CH}_{3} \mathrm{CO}\right)$ & 244-284 & $\operatorname{SLE}(\text { org })^{\mathrm{d}}$ & 8 & 0.20 & Carta and Dernini (1983) \\
\hline acetic acid, acetone & $\mathrm{CH}_{\mathrm{n}}, \mathrm{COOH}, \mathrm{CH}_{\mathrm{n}} \mathrm{CO}$ & $\left(\mathrm{CH}_{3}\right)(\mathrm{COOH}),\left(\mathrm{CH}_{3}\right)\left(\mathrm{CH}_{3} \mathrm{CO}\right)$ & $245-283$ & SLE(org) ${ }^{d}$ & 5 & 0.20 & Carta and Dernini (1983) \\
\hline acetic acid, acetone & $\mathrm{CH}_{\mathrm{n}}, \mathrm{COOH}, \mathrm{CH}_{\mathrm{n}} \mathrm{CO}$ & $\left(\mathrm{CH}_{3}\right)(\mathrm{COOH}),\left(\mathrm{CH}_{3}\right)\left(\mathrm{CH}_{3} \mathrm{CO}\right)$ & $332-383$ & VLE & 10 & 1.00 & Othmer (1943) \\
\hline acetic acid, acetone & $\mathrm{CH}_{\mathrm{n}}, \mathrm{COOH}, \mathrm{CH}_{\mathrm{n}} \mathrm{CO}$ & $\left(\mathrm{CH}_{3}\right)(\mathrm{COOH}),\left(\mathrm{CH}_{3}\right)\left(\mathrm{CH}_{3} \mathrm{CO}\right)$ & 308 & VLE & 12 & 0.10 & Waradzin and Surovy (1975) \\
\hline acetic acid, acetone & $\mathrm{CH}_{\mathrm{n}}, \mathrm{COOH}, \mathrm{CH}_{\mathrm{n}} \mathrm{CO}$ & $\left(\mathrm{CH}_{3}\right)(\mathrm{COOH}),\left(\mathrm{CH}_{3}\right)\left(\mathrm{CH}_{3} \mathrm{CO}\right)$ & 318 & VLE & 11 & 0.10 & Waradzin and Surovy (1975) \\
\hline acetic acid, acetone & $\mathrm{CH}_{\mathrm{n}}, \mathrm{COOH}, \mathrm{CH}_{\mathrm{n}} \mathrm{CO}$ & $\left(\mathrm{CH}_{3}\right)(\mathrm{COOH}),\left(\mathrm{CH}_{3}\right)\left(\mathrm{CH}_{3} \mathrm{CO}\right)$ & 328 & VLE & 11 & 0.10 & Waradzin and Surovy (1975) \\
\hline acetic acid, 2-butanone & $\mathrm{CH}_{\mathrm{n}}, \mathrm{COOH}, \mathrm{CH}_{\mathrm{n}} \mathrm{CO}$ & $\left(\mathrm{CH}_{3}\right)(\mathrm{COOH}),\left(\mathrm{CH}_{3}\right)\left(\mathrm{CH}_{2}\right)\left(\mathrm{CH}_{3} \mathrm{CO}\right)$ & $242-290$ & SLE(org) ${ }^{\mathrm{d}}$ & 12 & 0.20 & Dallos et al. (1986) \\
\hline acetic acid, 2-butanone & $\mathrm{CH}_{\mathrm{n}}, \mathrm{COOH}, \mathrm{CH}_{\mathrm{n}} \mathrm{CO}$ & $\left(\mathrm{CH}_{3}\right)(\mathrm{COOH}),\left(\mathrm{CH}_{3}\right)\left(\mathrm{CH}_{2}\right)\left(\mathrm{CH}_{3} \mathrm{CO}\right)$ & $353-391$ & VLE & 40 & 1.00 & Fu et al. (1986) \\
\hline acetic acid, 2-butanone & $\mathrm{CH}_{\mathrm{n}}, \mathrm{COOH}, \mathrm{CH}_{\mathrm{n}} \mathrm{CO}$ & $\left(\mathrm{CH}_{3}\right)(\mathrm{COOH}),\left(\mathrm{CH}_{3}\right)\left(\mathrm{CH}_{2}\right)\left(\mathrm{CH}_{3} \mathrm{CO}\right)$ & $353-388$ & VLE & 22 & 0.00 & Xie et al. (2009) \\
\hline acetic acid, 2-butanone & $\mathrm{CH}_{\mathrm{n}}, \mathrm{COOH}, \mathrm{CH}_{\mathrm{n}} \mathrm{CO}$ & $\left(\mathrm{CH}_{3}\right)(\mathrm{COOH}),\left(\mathrm{CH}_{3}\right)\left(\mathrm{CH}_{2}\right)\left(\mathrm{CH}_{3} \mathrm{CO}\right)$ & 303 & VLE & 12 & 0.00 & Dallos et al. (1986) \\
\hline acetic acid, 2-butanone & $\mathrm{CH}_{\mathrm{n}}, \mathrm{COOH}, \mathrm{CH}_{\mathrm{n}} \mathrm{CO}$ & $\left(\mathrm{CH}_{3}\right)(\mathrm{COOH}),\left(\mathrm{CH}_{3}\right)\left(\mathrm{CH}_{2}\right)\left(\mathrm{CH}_{3} \mathrm{CO}\right)$ & 323 & VLE & 14 & 1.00 & Dallos et al. (1986) \\
\hline acetic acid, 2-butanone & $\mathrm{CH}_{\mathrm{n}}, \mathrm{COOH}, \mathrm{CH}_{\mathrm{n}} \mathrm{CO}$ & $\left(\mathrm{CH}_{3}\right)(\mathrm{COOH}),\left(\mathrm{CH}_{3}\right)\left(\mathrm{CH}_{2}\right)\left(\mathrm{CH}_{3} \mathrm{CO}\right)$ & 351 & VLE & 9 & 1.00 & Dallos et al. (1986) \\
\hline butanoic acid, acetone & $\mathrm{CH}_{\mathrm{n}}, \mathrm{COOH}, \mathrm{CH}_{\mathrm{n}} \mathrm{CO}$ & $\left(\mathrm{CH}_{3}\right)\left(\mathrm{CH}_{2}\right)_{2}(\mathrm{COOH}),\left(\mathrm{CH}_{3}\right)\left(\mathrm{CH}_{3} \mathrm{CO}\right)$ & $240-268$ & SLE(org) ${ }^{\mathrm{d}}$ & 12 & 0.20 & Proust and Fernandez (1986) \\
\hline butanoic acid, 2-butanone & $\mathrm{CH}_{\mathrm{n}}, \mathrm{COOH}, \mathrm{CH}_{\mathrm{n}} \mathrm{CO}$ & $\left(\mathrm{CH}_{3}\right)\left(\mathrm{CH}_{2}\right)_{2}(\mathrm{COOH}),\left(\mathrm{CH}_{3}\right)\left(\mathrm{CH}_{2}\right)\left(\mathrm{CH}_{3} \mathrm{CO}\right)$ & $240-268$ & SLE(org) ${ }^{d}$ & 12 & 0.20 & Proust and Fernandez (1986) \\
\hline butanoic acid, 2-butanone & $\mathrm{CH}_{\mathrm{n}}, \mathrm{COOH}, \mathrm{CH}_{\mathrm{n}} \mathrm{CO}$ & $\left(\mathrm{CH}_{3}\right)\left(\mathrm{CH}_{2}\right)_{2}(\mathrm{COOH}),\left(\mathrm{CH}_{3}\right)\left(\mathrm{CH}_{2}\right)\left(\mathrm{CH}_{3} \mathrm{CO}\right)$ & 343 & VLE & 9 & 1.00 & Rasmussen and Fredenslund (1977) \\
\hline butanoic acid, 2-butanone & $\mathrm{CH}_{\mathrm{n}}, \mathrm{COOH}, \mathrm{CH}_{\mathrm{n}} \mathrm{CO}$ & $\left(\mathrm{CH}_{3}\right)\left(\mathrm{CH}_{2}\right)_{2}(\mathrm{COOH}),\left(\mathrm{CH}_{3}\right)\left(\mathrm{CH}_{2}\right)\left(\mathrm{CH}_{3} \mathrm{CO}\right)$ & 353 & VLE & 10 & 1.00 & Rasmussen and Fredenslund (1977) \\
\hline acetic acid, diethyl ether & $\mathrm{CH}_{\mathrm{n}}, \mathrm{COOH}, \mathrm{CH}_{\mathrm{n}} \mathrm{O}$ & $\left(\mathrm{CH}_{3}\right)(\mathrm{COOH}),\left(\mathrm{CH}_{3}\right)_{2}\left(\mathrm{CH}_{2}\right) \mathrm{CH}_{2} \mathrm{O}$ & $207-289$ & $\operatorname{SLE}(\text { org })^{\mathrm{d}}$ & 40 & 0.20 & Pickering (1893) \\
\hline acetic acid, diethyl ether & $\mathrm{CH}_{\mathrm{n}}, \mathrm{COOH}, \mathrm{CH}_{\mathrm{n}} \mathrm{O}$ & $\left(\mathrm{CH}_{3}\right)(\mathrm{COOH}),\left(\mathrm{CH}_{3}\right)_{2}\left(\mathrm{CH}_{2}\right) \mathrm{CH}_{2} \mathrm{O}$ & 293-343 & VLE & 7 & 1.00 & Meehan and Murphy (1965) \\
\hline acetic acid, diethyl ether & $\mathrm{CH}_{\mathrm{n}}, \mathrm{COOH}, \mathrm{CH}_{\mathrm{n}} \mathrm{O}$ & $\left(\mathrm{CH}_{3}\right)(\mathrm{COOH}),\left(\mathrm{CH}_{3}\right)_{2}\left(\mathrm{CH}_{2}\right) \mathrm{CH}_{2} \mathrm{O}$ & $299-351$ & VLE & 7 & 1.00 & Meehan and Murphy (1965) \\
\hline acetic acid, diethyl ether & $\mathrm{CH}_{\mathrm{n}}, \mathrm{COOH}, \mathrm{CH}_{\mathrm{n}} \mathrm{O}$ & $\left(\mathrm{CH}_{3}\right)(\mathrm{COOH}),\left(\mathrm{CH}_{3}\right)_{2}\left(\mathrm{CH}_{2}\right) \mathrm{CH}_{2} \mathrm{O}$ & $304-360$ & VLE & 7 & 1.00 & Meehan and Murphy (1965) \\
\hline acetic acid, ethyl acetate & $\mathrm{CH}_{\mathrm{n}}, \mathrm{COOH}, \mathrm{CCOO}$ & $\left(\mathrm{CH}_{3}\right)(\mathrm{COOH}),\left(\mathrm{CH}_{3}\right)\left(\mathrm{CH}_{2}\right)\left(\mathrm{CH}_{3} \mathrm{COO}\right)$ & 323 & VLE & 9 & 1.00 & Miyamoto et al. (2001) \\
\hline $\begin{array}{l}\text { hexadecanoic acid } \\
\text { (palmitic acid), ethyl ac- } \\
\text { etate }\end{array}$ & $\mathrm{CH}_{\mathrm{n}}, \mathrm{COOH}, \mathrm{CCOO}$ & $\left(\mathrm{CH}_{3}\right)\left(\mathrm{CH}_{2}\right)_{14}(\mathrm{COOH}),\left(\mathrm{CH}_{3}\right)\left(\mathrm{CH}_{2}\right)\left(\mathrm{CH}_{3} \mathrm{COO}\right)$ & $243-273$ & SLE(org) $)^{d}$ & 4 & 0.20 & Kolb (1959) \\
\hline $\begin{array}{l}\text { octadecanoic acid } \\
\text { (stearic acid), ethyl acetate }\end{array}$ & $\mathrm{CH}_{\mathrm{n}}, \mathrm{COOH}, \mathrm{CCOO}$ & $\left(\mathrm{CH}_{3}\right)\left(\mathrm{CH}_{2}\right)_{16}(\mathrm{COOH}),\left(\mathrm{CH}_{3}\right)\left(\mathrm{CH}_{2}\right)\left(\mathrm{CH}_{3} \mathrm{COO}\right)$ & $253-283$ & SLE(org) ${ }^{\mathrm{d}}$ & 4 & 0.20 & Kolb (1959) \\
\hline acetic acid, benzene & $\mathrm{CH}_{\mathrm{n}}, \mathrm{COOH}, \mathrm{ACH}_{\mathrm{n}}$ & $\left(\mathrm{CH}_{3}\right)(\mathrm{COOH}),(\mathrm{ACH})_{6}$ & $264-289$ & $\operatorname{SLE}(\text { org })^{\mathrm{d}}$ & 20 & 0.20 & Roloff (1895) \\
\hline acetic acid, benzene & $\mathrm{CH}_{\mathrm{n}}, \mathrm{COOH}, \mathrm{ACH}_{\mathrm{n}}$ & $\left(\mathrm{CH}_{3}\right)(\mathrm{COOH}),(\mathrm{ACH})_{6}$ & $274-289$ & SLE(org) ${ }^{\mathrm{d}}$ & 8 & 0.20 & Roloff (1895) \\
\hline acetic acid, benzene & $\mathrm{CH}_{\mathrm{n}}, \mathrm{COOH}, \mathrm{ACH}_{\mathrm{n}}$ & $\left(\mathrm{CH}_{3}\right)(\mathrm{COOH}),(\mathrm{ACH})_{6}$ & 313 & VLE & 9 & 0.20 & Miyamoto et al. (2000) \\
\hline acetic acid, benzene & $\mathrm{CH}_{\mathrm{n}}, \mathrm{COOH}, \mathrm{ACH}_{\mathrm{n}}$ & $\left(\mathrm{CH}_{3}\right)(\mathrm{COOH}),(\mathrm{ACH})_{6}$ & $353-387$ & VLE & 15 & 1.00 & Haughton (1967) \\
\hline acetic acid, benzene & $\mathrm{CH}_{\mathrm{n}}, \mathrm{COOH}, \mathrm{ACH}_{\mathrm{n}}$ & $\left(\mathrm{CH}_{3}\right)(\mathrm{COOH}),(\mathrm{ACH})_{6}$ & $296-322$ & VLE & 12 & 1.00 & Carta et al. (1979) \\
\hline acetic acid, acetaldehyde & $\mathrm{CH}_{\mathrm{n}}, \mathrm{COOH}, \mathrm{CHO}$ & $\left(\mathrm{CH}_{3}\right)(\mathrm{COOH}),\left(\mathrm{CH}_{3}\right)(\mathrm{CHO})$ & $295-386$ & VLE & 33 & 1.00 & Shanghai-Inst. and Zhejiang (1978) \\
\hline acetic acid, butyraldehyde & $\mathrm{CH}_{\mathrm{n}}, \mathrm{COOH}, \mathrm{CHO}$ & $\left(\mathrm{CH}_{3}\right)(\mathrm{COOH}),\left(\mathrm{CH}_{3}\right)\left(\mathrm{CH}_{2}\right)_{2}(\mathrm{CHO})$ & 323 & VLE & 9 & 1.00 & Miyamoto et al. (2001) \\
\hline $\begin{array}{l}\text { propanoic acid, butyralde- } \\
\text { hyde }\end{array}$ & $\mathrm{CH}_{\mathrm{n}}, \mathrm{COOH}, \mathrm{CHO}$ & $\left(\mathrm{CH}_{3}\right)\left(\mathrm{CH}_{2}\right)(\mathrm{COOH}),\left(\mathrm{CH}_{3}\right)\left(\mathrm{CH}_{2}\right)_{2}(\mathrm{CHO})$ & 323 & VLE & 9 & 1.00 & Miyamoto et al. (2001) \\
\hline $\begin{array}{l}\text { acetone, } \\
\text { 2-methoxy-2- } \\
\text { methylpropane }\end{array}$ & $\mathrm{CH}_{\mathrm{n}}, \mathrm{CH}_{\mathrm{n}} \mathrm{CO}, \mathrm{CH}_{\mathrm{n}} \mathrm{O}$ & $\left(\mathrm{CH}_{3}\right)\left(\mathrm{CH}_{3} \mathrm{CO}\right),\left(\mathrm{CH}_{3}\right)_{3}(\mathrm{C})\left(\mathrm{CH}_{3} \mathrm{O}\right)$ & $322-326$ & VLE & 19 & 1.00 & Mejía et al. (2008) \\
\hline $\begin{array}{l}\text { 2-butanone, } \\
\text { ethoxyethanol }\end{array}$ & $\begin{array}{l}\mathrm{CH}_{n}, \mathrm{CH}_{\mathrm{n}}^{[\mathrm{OH}]}, \mathrm{CH}_{\mathrm{n}} \mathrm{CO}, \mathrm{CH}_{\mathrm{n}} \mathrm{O} \text {, } \\
\mathrm{OH}\end{array}$ & $\begin{array}{l}\left(\mathrm{CH}_{3}\right)\left(\mathrm{CH}_{2}\right)\left(\mathrm{CH}_{3} \mathrm{CO}\right) \\
\left(\mathrm{CH}_{3}\right)\left(\mathrm{CH}_{2}\right)\left(\mathrm{CH}_{2}^{[\mathrm{OH}]}\right)\left(\mathrm{CH}_{2} \mathrm{O}\right)(\mathrm{OH})\end{array}$ & 330 & VLE & 9 & 1.00 & Naumann and Wagner (1986) \\
\hline acetone, ethyl acetate & $\mathrm{CH}_{\mathrm{n}}, \mathrm{CH}_{\mathrm{n}} \mathrm{CO}, \mathrm{CCOO}$ & $\left(\mathrm{CH}_{3}\right)\left(\mathrm{CH}_{3} \mathrm{CO}\right),\left(\mathrm{CH}_{3}\right)\left(\mathrm{CH}_{2}\right)\left(\mathrm{CH}_{3} \mathrm{COO}\right)$ & $330-348$ & VLE & 16 & 1.00 & Subrahmanyam and Murty (1964) \\
\hline acetone, ethyl acetate & $\mathrm{CH}_{\mathrm{n}}, \mathrm{CH}_{\mathrm{n}} \mathrm{CO}, \mathrm{CCOO}$ & $\left(\mathrm{CH}_{3}\right)\left(\mathrm{CH}_{3} \mathrm{CO}\right),\left(\mathrm{CH}_{3}\right)\left(\mathrm{CH}_{2}\right)\left(\mathrm{CH}_{3} \mathrm{COO}\right)$ & $328-348$ & VLE & 16 & 1.00 & Gilburd et al. (1979) \\
\hline acetone, ethyl acetate & $\mathrm{CH}_{\mathrm{n}}, \mathrm{CH}_{\mathrm{n}} \mathrm{CO}, \mathrm{CCOO}$ & $\left(\mathrm{CH}_{3}\right)\left(\mathrm{CH}_{3} \mathrm{CO}\right),\left(\mathrm{CH}_{3}\right)\left(\mathrm{CH}_{2}\right)\left(\mathrm{CH}_{3} \mathrm{COO}\right)$ & $313-330$ & VLE & 12 & 1.00 & Gilburd et al. (1981) \\
\hline $\begin{array}{l}\text { acetone, octadecanoic acid } \\
\text { methyl ester (methyl } \\
\text { stearate) }\end{array}$ & $\mathrm{CH}_{\mathrm{n}}, \mathrm{CH}_{\mathrm{n}} \mathrm{CO}, \mathrm{CCOO}$ & $\left(\mathrm{CH}_{3}\right)\left(\mathrm{CH}_{3} \mathrm{CO}\right),\left(\mathrm{CH}_{3}\right)\left(\mathrm{CH}_{2}\right)_{16}\left(\mathrm{CH}_{3} \mathrm{COO}\right)$ & $265-311$ & SLE(org) ${ }^{\mathrm{d}}$ & 6 & 0.10 & Bailey et al. (1970) \\
\hline $\begin{array}{l}\text { acetone, octadecanoic acid } \\
\text { ethyl ester (ethyl stearate) }\end{array}$ & $\mathrm{CH}_{\mathrm{n}}, \mathrm{CH}_{\mathrm{n}} \mathrm{CO}, \mathrm{CCOO}$ & $\left(\mathrm{CH}_{3}\right)\left(\mathrm{CH}_{3} \mathrm{CO}\right),\left(\mathrm{CH}_{3}\right)_{2}\left(\mathrm{CH}_{2}\right)_{16}\left(\mathrm{CH}_{3} \mathrm{COO}\right)$ & $263-303$ & SLE(org) ${ }^{\mathrm{d}}$ & 5 & 0.20 & Bailey et al. (1970) \\
\hline acetone, benzene & $\mathrm{CH}_{\mathrm{n}}, \mathrm{CH}_{\mathrm{n}} \mathrm{CO}, \mathrm{ACH}_{\mathrm{n}}$ & $\left(\mathrm{CH}_{3}\right)\left(\mathrm{CH}_{3} \mathrm{CO}\right),(\mathrm{ACH})_{6}$ & 318 & VLE & 11 & 1.00 & Brown and Smith (1957) \\
\hline acetone, benzene & $\mathrm{CH}_{\mathrm{n}}, \mathrm{CH}_{\mathrm{n}} \mathrm{CO}, \mathrm{ACH}_{\mathrm{n}}$ & $\left(\mathrm{CH}_{3}\right)\left(\mathrm{CH}_{3} \mathrm{CO}\right),(\mathrm{ACH})_{6}$ & $330-348$ & VLE & 21 & 1.00 & Kurihara et al. (1998) \\
\hline 2-heptanone, benzene & $\mathrm{CH}_{\mathrm{n}}, \mathrm{CH}_{\mathrm{n}} \mathrm{CO}, \mathrm{ACH}_{\mathrm{n}}$ & $\left(\mathrm{CH}_{3}\right)\left(\mathrm{CH}_{2}\right)_{4}\left(\mathrm{CH}_{3} \mathrm{CO}\right),(\mathrm{ACH})_{6}$ & $228-279$ & SLE(org) ${ }^{\mathrm{d}}$ & 13 & 0.20 & Fiege et al. (1996) \\
\hline 2-heptanone, benzene & $\mathrm{CH}_{\mathrm{n}}, \mathrm{CH}_{\mathrm{n}} \mathrm{CO}, \mathrm{ACH}_{\mathrm{n}}$ & $\left(\mathrm{CH}_{3}\right)\left(\mathrm{CH}_{2}\right)_{4}\left(\mathrm{CH}_{3} \mathrm{CO}\right),(\mathrm{ACH})_{6}$ & $228-238$ & SLE(org) ${ }^{d}$ & 8 & 0.20 & Fiege et al. (1996) \\
\hline 3-heptanone, benzene & $\mathrm{CH}_{\mathrm{n}}, \mathrm{CH}_{\mathrm{n}} \mathrm{CO}, \mathrm{ACH}_{\mathrm{n}}$ & $\left(\mathrm{CH}_{3}\right)_{2}\left(\mathrm{CH}_{2}\right)_{3}\left(\mathrm{CH}_{2} \mathrm{CO}\right),(\mathrm{ACH})_{6}$ & $228-279$ & SLE(org) ${ }^{d}$ & 13 & 0.20 & Fiege et al. (1996) \\
\hline 3-heptanone, benzene & $\mathrm{CH}_{\mathrm{n}}, \mathrm{CH}_{\mathrm{n}} \mathrm{CO}, \mathrm{ACH}_{\mathrm{n}}$ & $\left(\mathrm{CH}_{3}\right)_{2}\left(\mathrm{CH}_{2}\right)_{3}\left(\mathrm{CH}_{2} \mathrm{CO}\right),(\mathrm{ACH})_{6}$ & $225-236$ & SLE (org) ${ }^{\mathrm{d}}$ & 8 & 0.20 & Fiege et al. (1996) \\
\hline 4-heptanone, benzene & $\mathrm{CH}_{\mathrm{n}}, \mathrm{CH}_{\mathrm{n}} \mathrm{CO}, \mathrm{ACH}_{\mathrm{n}}$ & $\left(\mathrm{CH}_{3}\right)_{2}\left(\mathrm{CH}_{2}\right)_{3}\left(\mathrm{CH}_{2} \mathrm{CO}\right),(\mathrm{ACH})_{6}$ & $227-241$ & SLE (org) ${ }^{\mathrm{d}}$ & 9 & 0.20 & Fiege et al. (1996) \\
\hline 4-heptanone, benzene & $\mathrm{CH}_{\mathrm{n}}, \mathrm{CH}_{\mathrm{n}} \mathrm{CO}, \mathrm{ACH}_{\mathrm{n}}$ & $\left(\mathrm{CH}_{3}\right)_{2}\left(\mathrm{CH}_{2}\right)_{3}\left(\mathrm{CH}_{2} \mathrm{CO}\right),(\mathrm{ACH})_{6}$ & $238-279$ & SLE (org) ${ }^{d}$ & 11 & 0.20 & Fiege et al. (1996) \\
\hline acetone, acetaldehyde & $\mathrm{CH}_{\mathrm{n}}, \mathrm{CH}_{\mathrm{n}} \mathrm{CO}, \mathrm{CHO}$ & $\left(\mathrm{CH}_{3}\right)\left(\mathrm{CH}_{3} \mathrm{CO}\right),\left(\mathrm{CH}_{3}\right)(\mathrm{CHO})$ & 296-326 & VLE & 8 & 0.20 & Tikhonova et al. (1970) \\
\hline acetone, propionaldehyde & $\mathrm{CH}_{\mathrm{n}}, \mathrm{CH}_{\mathrm{n}} \mathrm{CO}, \mathrm{CHO}$ & $\left(\mathrm{CH}_{3}\right)\left(\mathrm{CH}_{3} \mathrm{CO}\right),\left(\mathrm{CH}_{3}\right)\left(\mathrm{CH}_{2}\right)(\mathrm{CHO})$ & $322-329$ & VLE & 13 & 1.00 & Danciu (1970) \\
\hline $\begin{array}{l}\text { 2-methoxyethanol, methyl } \\
\text { acetate }\end{array}$ & $\begin{array}{l}\mathrm{CH}_{\mathrm{n}}, \mathrm{CH}_{\mathrm{n}}^{[\mathrm{OH}]}, \mathrm{OH}, \quad \mathrm{CH}_{\mathrm{n}} \mathrm{O}, \\
\text { CCOO }\end{array}$ & $\begin{array}{l}\left(\mathrm{CH}_{2}\right)\left(\mathrm{CH}_{2}^{[\mathrm{OH}]}\right)\left(\mathrm{CH}_{3} \mathrm{O}\right)(\mathrm{OH}) \\
\left(\mathrm{CH}_{3}\right)\left(\mathrm{CH}_{3} \mathrm{COO}\right)\end{array}$ & 298 & VLE & 9 & 0.00 & Martin et al. (1994) \\
\hline $\begin{array}{l}\text { 2-methoxyethanol, ethyl } \\
\text { acetate }\end{array}$ & $\begin{array}{l}\mathrm{CH}_{\mathrm{n}}, \quad \mathrm{CH}_{\mathrm{n}}^{[\mathrm{OH}]}, \mathrm{OH}, \quad \mathrm{CH}_{\mathrm{n}} \mathrm{O}, \\
\mathrm{CCOO}\end{array}$ & $\begin{array}{l}\left(\mathrm{CH}_{2}\right)\left(\mathrm{CH}_{2}^{[\mathrm{OH}]}\right)\left(\mathrm{CH}_{3} \mathrm{O}\right)(\mathrm{OH}), \\
\left(\mathrm{CH}_{3}\right)\left(\mathrm{CH}_{2}\right)\left(\mathrm{CH}_{3} \mathrm{COO}\right)\end{array}$ & 343 & VLE & 13 & 0.20 & Chandak et al. (1977) \\
\hline $\begin{array}{l}\text { 2-methoxyethanol, ethyl } \\
\text { acetate }\end{array}$ & $\begin{array}{l}\mathrm{CH}_{\mathrm{n}}, \quad \mathrm{CH}_{\mathrm{n}}^{[\mathrm{OH}]}, \mathrm{OH}, \quad \mathrm{CH}_{\mathrm{n}} \mathrm{O}, \\
\text { CCOO }\end{array}$ & $\begin{array}{l}\left(\mathrm{CH}_{2}\right)\left(\mathrm{CH}_{2}^{[\mathrm{OH}]}\right)\left(\mathrm{CH}_{3} \mathrm{O}\right)(\mathrm{OH}) \\
\left(\mathrm{CH}_{3}\right)\left(\mathrm{CH}_{2}\right)\left(\mathrm{CH}_{3} \mathrm{COO}\right)\end{array}$ & 353 & VLE & 12 & 0.20 & Chandak et al. (1977) \\
\hline
\end{tabular}


Table 1. Continued.

\begin{tabular}{|c|c|c|c|c|c|c|c|c|}
\hline Organic compounds & Org. main groups & Chemical formula (subgroups) & & $T(\mathrm{~K})$ & Data type & $N_{d}$ & $w_{d}^{\text {init }}$ & Reference \\
\hline 2-methoxyethanol, ethyl acetate & $\begin{array}{l}\mathrm{CH}_{\mathrm{n}}, \mathrm{CH}_{\mathrm{n}}^{[\mathrm{OH}]}, \mathrm{OH}, \mathrm{CH}_{\mathrm{n}} \mathrm{O}, \\
\mathrm{CCOO}\end{array}$ & $\begin{array}{l}\left(\mathrm{CH}_{2}\right)\left(\mathrm{CH}_{2}^{[\mathrm{OH}]}\right)\left(\mathrm{CH}_{3} \mathrm{O}\right)(\mathrm{OH}), \\
\left(\mathrm{CH}_{3}\right)\left(\mathrm{CH}_{2}\right)\left(\mathrm{CH}_{3} \mathrm{COO}\right)\end{array}$ & & $351-395$ & VLE & 14 & 0.20 & Chandak et al. (1977) \\
\hline 2-ethoxyethanol, methyl acetate & $\begin{array}{l}\mathrm{CH}_{\mathrm{n}}, \mathrm{CH}_{\mathrm{n}}^{[\mathrm{OH}]}, \mathrm{OH}, \mathrm{CH}_{\mathrm{n}} \mathrm{O}, \\
\mathrm{CCOO}\end{array}$ & $\begin{array}{l}\left(\mathrm{CH}_{3}\right)\left(\mathrm{CH}_{2}\right)\left(\mathrm{CH}_{2}^{[\mathrm{OH}]}\right)\left(\mathrm{CH}_{2} \mathrm{O}\right)(\mathrm{OH}), \\
\left(\mathrm{CH}_{3}\right)\left(\mathrm{CH}_{3} \mathrm{COO}\right)\end{array}$ & & 298 & VLE & 9 & 0.00 & Martin et al. (1994) \\
\hline 2-ethoxyethanol, ethyl acetate & $\begin{array}{l}\mathrm{CH}_{\mathrm{n}}, \mathrm{CH}_{\mathrm{n}}^{[\mathrm{OH}]}, \mathrm{OH}, \mathrm{CH}_{\mathrm{n}} \mathrm{O}, \\
\text { CCOO }\end{array}$ & $\begin{array}{l}\left(\mathrm{CH}_{3}\right)\left(\mathrm{CH}_{2}\right)\left(\mathrm{CH}_{2}^{[\mathrm{OH}]}\right)\left(\mathrm{CH}_{2} \mathrm{O}\right)(\mathrm{OH}), \\
\left(\mathrm{CH}_{3}\right)\left(\mathrm{CH}_{2}\right)\left(\mathrm{CH}_{3} \mathrm{COO}\right)\end{array}$ & & $351-402$ & VLE & 17 & 0.20 & Thorat and Nageshwar (1988) \\
\hline diethyl ether, benzene & $\mathrm{CH}_{\mathrm{n}}, \mathrm{CH}_{\mathrm{n}} \mathrm{O}, \mathrm{ACH}_{\mathrm{n}}$ & $\left(\mathrm{CH}_{3}\right)_{2}\left(\mathrm{CH}_{2}\right) \mathrm{CH}_{2} \mathrm{O},(\mathrm{ACH})_{6}$ & & $197-278$ & SLE(org) ${ }^{\mathrm{d}}$ & 37 & 0.20 & Pickering (1893) \\
\hline 2-butoxyethanol, benzene & $\begin{array}{l}\mathrm{CH}_{\mathrm{n}}, \mathrm{CH}_{\mathrm{n}} \mathrm{O}, \mathrm{CH}_{\mathrm{n}}^{[\mathrm{OH}]}, \mathrm{OH}, \\
\mathrm{ACH}_{\mathrm{n}}\end{array}$ & $\begin{array}{l}\left(\mathrm{CH}_{3}\right)\left(\mathrm{CH}_{2}\right)_{3}\left(\mathrm{CH}_{2}^{[\mathrm{OH}]}\right)\left(\mathrm{CH}_{2} \mathrm{O}\right)(\mathrm{OH}), \\
(\mathrm{ACH})_{6}\end{array}$ & & $217-279$ & SLE(org) ${ }^{d}$ & 18 & 0.20 & Negadi et al. (2006) \\
\hline 1-methoxy-2-propanol, benzene & $\begin{array}{l}\mathrm{CH}_{\mathrm{n}}, \mathrm{CH}_{\mathrm{n}}^{[\mathrm{OH}]}, \mathrm{OH}, \mathrm{CH}_{\mathrm{n}} \mathrm{O}, \\
\mathrm{ACH}_{\mathrm{n}}\end{array}$ & $\begin{array}{l}\left(\mathrm{CH}_{3}\right)\left(\mathrm{CH}_{2}\right)\left(\mathrm{CH}^{[\mathrm{OH}]}\right)\left(\mathrm{CH}_{3} \mathrm{O}\right)(\mathrm{OH}), \\
(\mathrm{ACH})_{6}\end{array}$ & & $220-279$ & SLE(org) ${ }^{\mathrm{d}}$ & 18 & 0.20 & Negadi et al. (2006) \\
\hline 2-ethoxyethanol, phenol & $\begin{array}{l}\mathrm{CH}_{\mathrm{n}}, \mathrm{CH}_{\mathrm{n}}^{[\mathrm{OH}]}, \mathrm{OH}, \mathrm{CH}_{\mathrm{n}} \mathrm{O}, \\
\mathrm{ACH}_{\mathrm{n}}, \mathrm{ACOH}\end{array}$ & $\begin{array}{l}\left(\mathrm{CH}_{3}\right)\left(\mathrm{CH}_{2}\right)\left(\mathrm{CH}_{2}^{[\mathrm{OH}]}\right)\left(\mathrm{CH}_{2} \mathrm{O}\right)(\mathrm{OH}), \\
(\mathrm{ACH})_{5}(\mathrm{ACOH})\end{array}$ & & 363 & VLE & 17 & 0.10 & Chylinski et al. (2001) \\
\hline 2-ethoxyethanol, phenol & $\begin{array}{l}\mathrm{CH}_{\mathrm{n}}, \mathrm{CH}_{\mathrm{n}}^{[\mathrm{OH}]}, \mathrm{OH}, \mathrm{CH}_{\mathrm{n}} \mathrm{O}, \\
\mathrm{ACH}_{\mathrm{n}}, \mathrm{ACOH}\end{array}$ & $\begin{array}{l}\left(\mathrm{CH}_{3}\right)\left(\mathrm{CH}_{2}\right)\left(\mathrm{CH}_{2}^{[\mathrm{OH}]}\right)\left(\mathrm{CH}_{2} \mathrm{O}\right)(\mathrm{OH}), \\
(\mathrm{ACH})_{5}(\mathrm{ACOH})\end{array}$ & & 373 & VLE & 17 & 0.10 & Chylinski et al. (2001) \\
\hline 2-ethoxyethanol, phenol & $\begin{array}{l}\mathrm{CH}_{\mathrm{n}}, \mathrm{CH}_{\mathrm{n}}^{[\mathrm{OH}]}, \mathrm{OH} \\
\mathrm{CH}_{\mathrm{n}} \mathrm{O}, \mathrm{ACH}_{\mathrm{n}} \\
\mathrm{ACOH}\end{array}$ & $\begin{array}{l}\left(\mathrm{CH}_{3}\right)\left(\mathrm{CH}_{2}\right)\left(\mathrm{CH}_{2}^{[\mathrm{OH}]}\right)\left(\mathrm{CH}_{2} \mathrm{O}\right) \\
(\mathrm{ACH})_{5}(\mathrm{ACOH})\end{array}$ & $(\mathrm{OH})$, & 383 & VLE & 17 & 0.10 & Chylinski et al. (2001) \\
\hline ethyl acetate, benzene & $\mathrm{CH}_{\mathrm{n}}, \mathrm{CCOO}, \mathrm{ACH}_{\mathrm{n}}$ & $\left(\mathrm{CH}_{3}\right)\left(\mathrm{CH}_{2}\right)\left(\mathrm{CH}_{3} \mathrm{COO}\right),(\mathrm{ACH})_{6}$ & & $350-353$ & VLE & 19 & 1.00 & Carr and Kropholler (1962) \\
\hline $\begin{array}{l}\text { ethyl acetate, 2-hydroxybenzoic } \\
\text { acid }\end{array}$ & $\begin{array}{l}\mathrm{CH}_{\mathrm{n}}, \mathrm{CCOO}, \mathrm{ACH}_{\mathrm{n}} \\
\mathrm{ACOH}, \mathrm{COOH}\end{array}$ & $\begin{array}{l}\left(\mathrm{CH}_{3}\right)\left(\mathrm{CH}_{2}\right)\left(\mathrm{CH}_{3} \mathrm{COO}\right) \\
(\mathrm{ACH})_{4}(\mathrm{AC})(\mathrm{ACOH})(\mathrm{COOH})\end{array}$ & & $298-348$ & SLE & 11 & 1.00 & Shalmashi and Eliassi (2008) \\
\hline methyl acetate, butyraldehyde & $\mathrm{CH}_{\mathrm{n}}, \mathrm{CCOO}, \mathrm{CHO}$ & $\left(\mathrm{CH}_{3}\right)\left(\mathrm{CH}_{3} \mathrm{COO}\right),\left(\mathrm{CH}_{3}\right)\left(\mathrm{CH}_{2}\right)_{2}(\mathrm{CHO})$ & & 313 & VLE & 15 & 1.00 & Radnai et al. (1987) \\
\hline methyl acetate, butyraldehyde & $\mathrm{CH}_{\mathrm{n}}, \mathrm{CCOO}, \mathrm{CHO}$ & $\left(\mathrm{CH}_{3}\right)\left(\mathrm{CH}_{3} \mathrm{COO}\right),\left(\mathrm{CH}_{3}\right)\left(\mathrm{CH}_{2}\right)_{2}(\mathrm{CHO})$ & & 323 & VLE & 15 & 1.00 & Radnai et al. (1987) \\
\hline benzene, butyraldehyde & $\mathrm{CH}_{\mathrm{n}}, \mathrm{ACH}_{\mathrm{n}}, \mathrm{CHO}$ & $(\mathrm{ACH})_{6},\left(\mathrm{CH}_{3}\right)\left(\mathrm{CH}_{2}\right)_{2}(\mathrm{CHO})$ & & 353 & VLE & 5 & 1.00 & Leu et al. (1989) \\
\hline benzene, butyraldehyde & $\mathrm{CH}_{\mathrm{n}}, \mathrm{ACH}_{\mathrm{n}}, \mathrm{CHO}$ & $(\mathrm{ACH})_{6},\left(\mathrm{CH}_{3}\right)\left(\mathrm{CH}_{2}\right)_{2}(\mathrm{CHO})$ & & 393 & VLE & 6 & 1.00 & Leu et al. (1989) \\
\hline
\end{tabular}

a Derived water activity data from differential scanning calorimetry (DSC) measurements at homogeneous freezing temperatures.

b M5 is a mixture of dicarboxylic acids consisting of the following: malic acid (2) + malonic acid (3) + maleic acid (4) + glutaric acid (5) + methylsuccinic acid (6).

c The chemical subgroup formulas of the M5 components are given in the table for the individual components, except for maleic acid,

for which the subgroup formula is as follows: $(\mathrm{CH}=\mathrm{CH})(\mathrm{COOH})_{2}$.

d SLE data where the equilibrium is with respect to an organic compound in a solid (crystalline) state.

e Derived water activity data from total pressure measurements, for more information we refer to Ganbavale et al. (2014).

${ }^{\mathrm{f}}$ Derived water activity data from electrodynamic balance (EDB) measurements, for more information we refer to Ganbavale et al. (2014).

composition. Consequently, at maximum two data points for each temperature level can be acquired for binary systems, corresponding to the points on the melting curves of the two components. However, most data sets collected provide only data for one component forming a solid in equilibrium with the remaining liquid solution. In many of these cases, hexagonal water ice is the solid phase. Since the temperature dependence of water activity $\left(a_{\mathrm{w}}\right)$ of aqueous solutions in equilibrium with ice is well known, an accurate determination of the activity coefficients $\left(\gamma_{\mathrm{w}}^{(x)}=\frac{a_{\mathrm{w}}}{x_{\mathrm{w}}}\right)$ of water as a function of solution composition and temperature using SLE data is possible. At SLE, the activity of water in a solution with organic mole fraction $x_{\text {org }}$ at thermodynamic equilibrium with ice, $a_{\mathrm{w}}^{\mathrm{SLE}}(T, p)$, is given by (Koop et al., 2000):

$a_{\mathrm{w}}^{\mathrm{SLE}}(T, p)=\exp \left[\frac{\mu_{\mathrm{w}}^{\mathrm{S}}(T, p)-\mu_{w}^{\circ, \mathrm{L}}(T, p)}{R T}\right]$

where $\mu_{\mathrm{w}}^{\mathrm{S}}(T)$ and $\mu_{w}^{\circ, \mathrm{L}}(T)$ are the pressure and temperature dependent chemical potentials of ice (superscript $S$ ) and pure liquid water (superscript $\circ, \mathrm{L}$ ), respectively. At ambient pressures, neglecting the pressure dependence of the liquids and solids is well justified.

$$
\begin{aligned}
\mu_{\mathrm{w}}^{\mathrm{S}}(T) & -\mu_{w}^{\circ, \mathrm{L}}(T)=210368+131.438 T \\
& -3.32373 \times 10^{6} T^{-1}-41729.1 \ln (T) .
\end{aligned}
$$

The parameterisation in Eq. (18) represents the thermodynamically consistent function for use with Eq. (17) valid at low (ambient) pressure in the temperature range $150<T<$ $273 \mathrm{~K}$ (Koop et al., 2000).

The activity of a dissolved organic component in equilibrium with its pure crystalline solid can be calculated using the following relationship (Prausnitz et al., 1986; Dománska et al., 2009):

$$
\begin{aligned}
\ln x_{i} \gamma_{i}^{\mathrm{SLE}} & =-\frac{\Delta h_{\mathrm{m}, i}}{R T}\left(1-\frac{T}{T_{\mathrm{m}, i}}\right)-\frac{\Delta h_{\mathrm{tr}, i}}{R T}\left(1-\frac{T}{T_{\mathrm{tr}, i}}\right) \\
& +\frac{\Delta c_{p, \mathrm{~m}, i}}{R}\left[\left(1-\frac{T}{T_{\mathrm{m}, i}}\right)+\ln \frac{T}{T_{\mathrm{m}, i}}\right],
\end{aligned}
$$

where $\Delta h_{\mathrm{m}, i}$ is the molar enthalpy of fusion (melting, subscript $\mathrm{m}), \Delta h_{\mathrm{tr}, i}$ is the molar enthalpy of a certain solid-solid phase transition, $\Delta c_{p, \mathrm{~m}, i}$ is the molar heat capacity change upon fusion at constant pressure, $T_{\text {tr }}$ is the solid-solid phase transition temperature and $T_{\mathrm{m}, i}$ the melting temperature of pure component $i$. The second term is only of significance 
Table 2. Matrix of AIOMFAC short-range group interaction parameters. Parameter values for $a(i, j)$ (units of K) are from the literature ${ }^{\mathrm{a}}$, $b(i, j)$ (units of $\mathrm{K})$, and $c(i, j)$ (dimensionless) are determined in this study.

\begin{tabular}{|c|c|c|c|c|c|c|c|c|c|c|}
\hline $\begin{array}{l}\text { group no. } \\
i \downarrow\end{array}$ & $\begin{array}{l}j \rightarrow \\
\text { main groups }\end{array}$ & & $\begin{array}{r}1 \\
\mathrm{CH}_{\mathrm{n}}\end{array}$ & $\begin{array}{r}2 \\
\mathrm{C}=\mathrm{C}\end{array}$ & $\begin{array}{r}3 \\
\mathrm{ACH}_{\mathrm{n}}\end{array}$ & $\begin{array}{r}7 \\
\mathrm{H}_{2} \mathrm{O}\end{array}$ & $\begin{array}{r}8 \\
\mathrm{ACOH}\end{array}$ & $\begin{array}{r}9 \\
\mathrm{CH}_{\mathrm{n}} \mathrm{CO}\end{array}$ & $\begin{array}{r}10 \\
\text { CHO[aldehyde] }\end{array}$ & $\begin{array}{r}11 \\
\mathrm{CCOO}\end{array}$ \\
\hline 1 & $\mathrm{CH}_{\mathrm{n}}$ & $\begin{array}{l}a(i, j): \\
b(i, j): \\
c(i, j):\end{array}$ & $\begin{array}{r}0.0 \\
0.0 \\
0.0\end{array}$ & $\begin{array}{r}8.6020 \times 10^{1} \\
0.0^{\mathrm{b}} \\
0.0^{\mathrm{b}}\end{array}$ & $\begin{array}{r}6.1130 \times 10^{1} \\
2.0000 \times 10^{2} \\
3.0257 \times 10^{-1}\end{array}$ & $\begin{array}{l}1.3180 \times 10^{3} \\
1.2481 \times 10^{3} \\
5.2720 \times 10^{0}\end{array}$ & $\begin{array}{l}1.3330 \times 10^{3} \\
1.3330 \times 10^{3} \\
3.1475 \times 10^{0}\end{array}$ & $\begin{array}{r}4.7640 \times 10^{2} \\
-4.7640 \times 10^{2} \\
1.9056 \times 10^{0}\end{array}$ & $\begin{array}{r}6.7700 \times 10^{2} \\
2.0000 \times 10^{2} \\
4.2165 \times 10^{-1}\end{array}$ & $\begin{array}{r}2.3210 \times 10^{2} \\
-2.0762 \times 10^{1} \\
9.2840 \times 10^{-1}\end{array}$ \\
\hline 2 & $\mathrm{C}=\mathrm{C}$ & $\begin{array}{l}a(i, j): \\
b(i, j): \\
c(i, j):\end{array}$ & $\begin{array}{r}-3.5360 \times 10^{1} \\
0.0^{\mathrm{b}} \\
0.0^{\mathrm{b}}\end{array}$ & $\begin{array}{l}0.0 \\
0.0 \\
0.0\end{array}$ & $\begin{array}{r}3.8810 \times 10^{1} \\
0.0^{\mathrm{b}} \\
0.0^{\mathrm{b}}\end{array}$ & $\begin{array}{r}2.7060 \times 10^{2} \\
0.0^{\mathrm{b}} \\
0.0^{\mathrm{b}}\end{array}$ & $\begin{array}{r}5.2610 \times 10^{2} \\
0.0^{\mathrm{b}} \\
0.0^{\mathrm{b}}\end{array}$ & $\begin{array}{r}1.8260 \times 10^{2} \\
0.0^{\mathrm{b}} \\
0.0^{\mathrm{b}}\end{array}$ & $\begin{array}{r}4.4880 \times 10^{2} \\
0.0^{\mathrm{b}} \\
0.0^{\mathrm{b}}\end{array}$ & $\begin{array}{r}3.7850 \times 10^{1} \\
0.0^{b} \\
0.0^{b}\end{array}$ \\
\hline 3 & $\mathrm{ACH}_{\mathrm{n}}$ & $\begin{array}{l}a(i, j): \\
b(i, j): \\
c(i, j):\end{array}$ & $\begin{array}{r}-1.1120 \times 10^{1} \\
1.9504 \times 10^{1} \\
8.0000 \times 10^{-1}\end{array}$ & $\begin{array}{r}3.4460 \times 10^{0} \\
0.0^{\mathrm{b}} \\
0.0^{\mathrm{b}}\end{array}$ & $\begin{array}{l}0.0 \\
0.0 \\
0.0\end{array}$ & $\begin{array}{l}9.0380 \times 10^{2} \\
9.0380 \times 10^{2} \\
2.3228 \times 10^{0}\end{array}$ & $\begin{array}{r}1.3290 \times 10^{3} \\
-1.3290 \times 10^{3} \\
-5.3160 \times 10^{0}\end{array}$ & $\begin{array}{r}2.5770 \times 10^{1} \\
-1.8718 \times 10^{2} \\
0.0^{b}\end{array}$ & $\begin{array}{r}3.4730 \times 10^{2} \\
-3.4730 \times 10^{2} \\
0.0^{b}\end{array}$ & $\begin{array}{r}5.9940 \times 10^{0} \\
0.0^{\mathrm{b}} \\
0.0^{\mathrm{b}}\end{array}$ \\
\hline 7 & $\mathrm{H}_{2} \mathrm{O}$ & $\begin{array}{l}a(i, j): \\
b(i, j): \\
c(i, j):\end{array}$ & $\begin{aligned} 3.0000 & \times 10^{2} \\
2.07691 & \times 10^{0} \\
-1.2000 & \times 10^{0}\end{aligned}$ & $\begin{array}{r}4.9610 \times 10^{2} \\
0.0^{\mathrm{b}} \\
0.0^{\mathrm{b}}\end{array}$ & $\begin{array}{r}3.6230 \times 10^{2} \\
-3.6230 \times 10^{2} \\
1.2682 \times 10^{0}\end{array}$ & $\begin{array}{r}0.0 \\
0.0 \\
0.0\end{array}$ & $\begin{array}{r}3.2450 \times 10^{2} \\
2.6456 \times 10^{2} \\
-1.2980 \times 10^{0}\end{array}$ & $\begin{array}{r}-1.9540 \times 10^{2} \\
4.5845 \times 10^{1} \\
-8.0000 \times 10^{-1}\end{array}$ & $\begin{array}{r}-1.1600 \times 10^{2} \\
-2.9366 \times 10^{1} \\
0.0^{b}\end{array}$ & $\begin{array}{r}7.2870 \times 10^{1} \\
-1.3512 \times 10^{1} \\
-8.0000 \times 10^{-1}\end{array}$ \\
\hline 8 & $\mathrm{ACOH}$ & $\begin{array}{l}a(i, j): \\
b(i, j): \\
c(i, j):\end{array}$ & $\begin{array}{r}2.7580 \times 10^{2} \\
2.7580 \times 10^{2} \\
9.7679 \times 10^{-1}\end{array}$ & $\begin{array}{r}2.1750 \times 10^{2} \\
0.0^{b} \\
0.0^{b}\end{array}$ & $\begin{array}{r}2.5340 \times 10^{1} \\
2.0000 \times 10^{2} \\
-4.9742 \times 10^{-1}\end{array}$ & $\begin{array}{r}-6.0180 \times 10^{2} \\
9.8265 \times 10^{1} \\
-2.0012 \times 10^{-1}\end{array}$ & $\begin{array}{r}0.0 \\
0.0 \\
0.0\end{array}$ & $\begin{array}{r}-3.5610 \times 10^{2} \\
3.5610 \times 10^{2} \\
0.0^{b}\end{array}$ & $\begin{array}{r}-2.7110 \times 10^{2} \\
0.0^{\mathrm{b}} \\
0.0^{\mathrm{b}}\end{array}$ & $\begin{array}{r}-4.4940 \times 10^{2} \\
3.5689 \times 10^{2} \\
0.0^{b}\end{array}$ \\
\hline 9 & $\mathrm{CH}_{\mathrm{n}} \mathrm{CO}$ & $\begin{array}{l}a(i, j): \\
b(i, j): \\
c(i, j):\end{array}$ & $\begin{array}{r}2.6760 \times 10^{1} \\
-5.0597 \times 10^{1} \\
-8.0000 \times 10^{-1}\end{array}$ & $\begin{array}{r}4.2920 \times 10^{1} \\
0.0^{\mathrm{b}} \\
0.0^{\mathrm{b}}\end{array}$ & $\begin{array}{r}1.4010 \times 10^{2} \\
-1.8962 \times 10^{1} \\
0.0^{b}\end{array}$ & $\begin{aligned} 4.7250 & \times 10^{2} \\
1.1460 & \times 10^{2} \\
-1.8900 & \times 10^{0}\end{aligned}$ & $\begin{array}{r}-1.3310 \times 10^{2} \\
2.0000 \times 10^{2} \\
0.0^{\mathrm{b}}\end{array}$ & $\begin{array}{l}0.0 \\
0.0 \\
0.0\end{array}$ & $\begin{array}{r}-3.7360 \times 10^{1} \\
0.0^{\mathrm{b}} \\
0.0^{\mathrm{b}}\end{array}$ & $\begin{array}{r}-2.1370 \times 10^{2} \\
2.1370 \times 10^{2} \\
0.0^{b}\end{array}$ \\
\hline 10 & $\mathrm{CHO}$ [aldehyde] & $\begin{array}{l}a(i, j): \\
b(i, j): \\
c(i, j):\end{array}$ & $\begin{aligned} & 5.0570 \times 10^{2} \\
& 5.0569 \times 10^{2} \\
&-2.0228 \times 10^{0}\end{aligned}$ & $\begin{array}{r}5.6300 \times 10^{1} \\
0.0^{b} \\
0.0^{b}\end{array}$ & $\begin{array}{r}2.3390 \times 10^{1} \\
-2.0000 \times 10^{2} \\
0.0^{b}\end{array}$ & $\begin{array}{r}4.8080 \times 10^{2} \\
4.8080 \times 10^{2} \\
0.0^{b}\end{array}$ & $\begin{array}{r}-1.5560 \times 10^{2} \\
0.0^{b} \\
0.0^{b}\end{array}$ & $\begin{array}{r}1.2800 \times 10^{2} \\
0.0^{\mathrm{b}} \\
0.0^{\mathrm{b}}\end{array}$ & $\begin{array}{r}0.0 \\
0.0 \\
0.0\end{array}$ & $\begin{array}{r}-1.1030 \times 10^{2} \\
0.0^{\mathrm{b}} \\
0.0^{\mathrm{b}}\end{array}$ \\
\hline 11 & $\mathrm{CCOO}$ & $\begin{array}{l}a(i, j): \\
b(i, j): \\
c(i, j):\end{array}$ & $\begin{array}{r}1.1480 \times 10^{2} \\
2.0000 \times 10^{2} \\
8.0000 \times 10^{-1}\end{array}$ & $\begin{array}{r}1.3210 \times 10^{2} \\
0.0^{\mathrm{b}} \\
0.0^{\mathrm{b}}\end{array}$ & $\begin{array}{r}8.5840 \times 10^{1} \\
0.0^{b} \\
0.0^{b}\end{array}$ & $\begin{array}{r}2.0080 \times 10^{2} \\
7.7173 \times 10^{1} \\
-8.0320 \times 10^{-1}\end{array}$ & $\begin{array}{r}-3.6720 \times 10^{1} \\
1.9950 \times 10^{2} \\
0.0^{b}\end{array}$ & $\begin{array}{r}3.7220 \times 10^{2} \\
-1.1627 \times 10^{2} \\
0.0^{b}\end{array}$ & $\begin{array}{r}1.8510 \times 10^{2} \\
0.0^{\mathrm{b}} \\
0.0^{\mathrm{b}}\end{array}$ & $\begin{array}{r}0.0 \\
0.0 \\
0.0\end{array}$ \\
\hline 13 & $\mathrm{CH}_{\mathrm{n}} \mathrm{O}$ [ether] & $\begin{array}{l}a(i, j): \\
b(i, j): \\
c(i, j):\end{array}$ & $\begin{array}{r}8.3360 \times 10^{1} \\
2.0000 \times 10^{2} \\
8.0000 \times 10^{-1}\end{array}$ & $\begin{array}{r}2.6510 \times 10^{1} \\
0.0^{\mathrm{b}} \\
0.0^{\mathrm{b}}\end{array}$ & $\begin{array}{r}5.2130 \times 10^{1} \\
-2.0000 \times 10^{2} \\
8.0000 \times 10^{-1}\end{array}$ & $\begin{array}{l}-3.1470 \times 10^{2} \\
-3.1470 \times 10^{2} \\
-1.2588 \times 10^{0}\end{array}$ & $\begin{array}{r}-1.7850 \times 10^{2} \\
0.0^{\mathrm{b}} \\
0.0^{\mathrm{b}}\end{array}$ & $\begin{array}{r}1.9110 \times 10^{2} \\
0.0^{\mathrm{b}} \\
0.0^{\mathrm{b}}\end{array}$ & $\begin{array}{r}-7.8380 \times 10^{0} \\
0.0^{\mathrm{b}} \\
0.0^{\mathrm{b}}\end{array}$ & $\begin{array}{r}4.6130 \times 10^{2} \\
-4.5906 \times 10^{2} \\
-9.6038 \times 10^{-1}\end{array}$ \\
\hline 65 & $\mathrm{COOH}$ & $\begin{array}{l}a(i, j): \\
b(i, j): \\
c(i, j):\end{array}$ & $\begin{array}{l}3.1530 \times 10^{2} \\
3.1530 \times 10^{2} \\
1.2612 \times 10^{0}\end{array}$ & $\begin{array}{r}1.2640 \times 10^{3} \\
0.0^{\mathrm{b}} \\
0.0^{\mathrm{b}}\end{array}$ & $\begin{array}{r}6.2320 \times 10^{1} \\
-4.4703 \times 10^{0} \\
8.0000 \times 10^{-1}\end{array}$ & $\begin{array}{r}-1.4588 \times 10^{2} \\
4.7584 \times 10^{1} \\
-8.0000 \times 10^{-1}\end{array}$ & $\begin{array}{r}-1.1000 \times 10^{1} \\
2.0000 \times 10^{2} \\
4.0284 \times 10^{-2}\end{array}$ & $\begin{array}{l}-2.9780 \times 10^{2} \\
-7.4212 \times 10^{1} \\
-1.1912 \times 10^{0}\end{array}$ & $\begin{array}{r}-1.6550 \times 10^{2} \\
2.0332 \times 10^{1} \\
8.0000 \times 10^{-1}\end{array}$ & $\begin{aligned} &-2.5630 \times 10^{2} \\
& 2.5630 \times 10^{2} \\
& 0.0^{b}\end{aligned}$ \\
\hline 66 & $\mathrm{CH}_{\mathrm{n}}^{[\mathrm{alc}]}$ & $\begin{array}{l}a(i, j): \\
b(i, j): \\
c(i, j):\end{array}$ & $\begin{array}{l}0.0^{\mathrm{c}} \\
0.0^{\mathrm{c}} \\
0.0^{\mathrm{c}}\end{array}$ & $\begin{array}{r}8.6020 \times 10^{1} \\
0.0^{b} \\
0.0^{b}\end{array}$ & $\begin{array}{r}6.1130 \times 10^{1} \\
2.0000 \times 10^{2} \\
3.0257 \times 10^{-1}\end{array}$ & $\begin{array}{l}1.8900 \times 10^{3} \\
1.8900 \times 10^{3} \\
7.5600 \times 10^{0}\end{array}$ & $\begin{array}{l}1.3330 \times 10^{3} \\
1.3330 \times 10^{3} \\
3.1475 \times 10^{0}\end{array}$ & $\begin{aligned} 4.7640 & \times 10^{2} \\
-4.7640 & \times 10^{2} \\
1.9056 & \times 10^{0}\end{aligned}$ & $\begin{array}{r}6.7700 \times 10^{2} \\
2.0000 \times 10^{2} \\
4.2165 \times 10^{-1}\end{array}$ & $\begin{array}{r}2.3210 \times 10^{2} \\
-2.0762 \times 10^{1} \\
9.2840 \times 10^{-1}\end{array}$ \\
\hline 67 & $\mathrm{CH}_{\mathrm{n}}^{\text {[alc-tail] }}$ & $\begin{array}{l}a(i, j): \\
b(i, j): \\
c(i, j):\end{array}$ & $\begin{array}{l}0.0^{\mathrm{c}} \\
0.0^{\mathrm{c}} \\
0.0^{\mathrm{c}}\end{array}$ & $\begin{array}{r}8.6020 \times 10^{1} \\
0.0^{b} \\
0.0^{b}\end{array}$ & $\begin{array}{r}6.1130 \times 10^{1} \\
2.0000 \times 10^{2} \\
3.0257 \times 10^{-1}\end{array}$ & $\begin{array}{l}1.3250 \times 10^{3} \\
1.3250 \times 10^{3} \\
2.9603 \times 10^{0}\end{array}$ & $\begin{array}{l}1.3330 \times 10^{3} \\
1.3330 \times 10^{3} \\
3.1475 \times 10^{0}\end{array}$ & $\begin{array}{r}4.7640 \times 10^{2} \\
-4.7640 \times 10^{2} \\
1.9056 \times 10^{0}\end{array}$ & $\begin{array}{r}6.7700 \times 10^{2} \\
2.0000 \times 10^{2} \\
4.2165 \times 10^{-1}\end{array}$ & $\begin{array}{r}2.3210 \times 10^{2} \\
-2.0762 \times 10^{1} \\
9.2840 \times 10^{-1}\end{array}$ \\
\hline 68 & $\mathrm{CH}_{\mathrm{n}}^{[\mathrm{OH}]}$ & $\begin{array}{l}a(i, j): \\
b(i, j): \\
c(i, j):\end{array}$ & $\begin{array}{l}0.0^{\mathrm{c}} \\
0.0^{\mathrm{c}} \\
0.0^{\mathrm{c}}\end{array}$ & $\begin{array}{r}8.6020 \times 10^{1} \\
0.0^{b} \\
0.0^{b}\end{array}$ & $\begin{array}{r}6.1130 \times 10^{1} \\
2.0000 \times 10^{2} \\
3.0257 \times 10^{-1}\end{array}$ & $\begin{array}{r}2.3140 \times 10^{3} \\
-2.1510 \times 10^{3} \\
-1.6464 \times 10^{0}\end{array}$ & $\begin{array}{l}1.3330 \times 10^{3} \\
1.3330 \times 10^{3} \\
3.1475 \times 10^{0}\end{array}$ & $\begin{array}{r}4.7640 \times 10^{2} \\
-4.7640 \times 10^{2} \\
1.9056 \times 10^{0}\end{array}$ & $\begin{array}{r}6.7700 \times 10^{2} \\
2.0000 \times 10^{2} \\
4.2165 \times 10^{-1}\end{array}$ & $\begin{array}{r}2.3210 \times 10^{2} \\
-2.0762 \times 10^{1} \\
9.2840 \times 10^{-1}\end{array}$ \\
\hline 69 & $\mathrm{OH}$ & $\begin{array}{l}a(i, j): \\
b(i, j): \\
c(i, j):\end{array}$ & $\begin{array}{r}1.5640 \times 10^{2} \\
2.0000 \times 10^{2} \\
-8.0000 \times 10^{-1}\end{array}$ & $\begin{array}{r}4.5700 \times 10^{2} \\
0.0^{\mathrm{b}} \\
0.0^{\mathrm{b}}\end{array}$ & $\begin{array}{r}8.9600 \times 10^{1} \\
2.0000 \times 10^{2} \\
8.0000 \times 10^{-1}\end{array}$ & $\begin{array}{r}2.7640 \times 10^{2} \\
2.7640 \times 10^{2} \\
-1.1056 \times 10^{0}\end{array}$ & $\begin{array}{r}-2.5970 \times 10^{2} \\
2.5970 \times 10^{2} \\
9.7596 \times 10^{-1}\end{array}$ & $\begin{array}{r}8.4000 \times 10^{1} \\
1.9847 \times 10^{2} \\
8.0000 \times 10^{-1}\end{array}$ & $\begin{array}{r}-2.0360 \times 10^{2} \\
-2.0360 \times 10^{2} \\
-1.2530 \times 10^{-1}\end{array}$ & $\begin{array}{r}1.0110 \times 10^{2} \\
2.0000 \times 10^{2} \\
8.0000 \times 10^{-1}\end{array}$ \\
\hline
\end{tabular}


Table 2. Continued.

\begin{tabular}{|c|c|c|c|c|c|c|c|c|}
\hline $\begin{array}{l}\text { group no. } \\
i \downarrow\end{array}$ & $\begin{array}{l}j \rightarrow \\
\text { main groups }\end{array}$ & & $\begin{array}{r}13 \\
\mathrm{CH}_{\mathrm{n}} \mathrm{O}[\text { ether] }\end{array}$ & $\begin{array}{r}65 \\
\mathrm{COOH}\end{array}$ & $\mathrm{CH}_{\mathrm{n}}^{\text {[alc] }}$ & $\mathrm{CH}_{\mathrm{n}}^{\text {[alc-tail] }}$ & $\mathrm{CH}_{\mathrm{n}}^{[\mathrm{OH}]}$ & $\begin{array}{r}69 \\
\mathrm{OH}\end{array}$ \\
\hline 1 & $\mathrm{CH}_{\mathrm{n}}$ & $\begin{array}{l}a(i, j): \\
b(i, j): \\
c(i, j):\end{array}$ & $\begin{array}{r}2.5150 \times 10^{2} \\
-1.7943 \times 10^{2} \\
5.5434 \times 10^{-1}\end{array}$ & $\begin{array}{l}6.6350 \times 10^{2} \\
6.6350 \times 10^{2} \\
2.6540 \times 10^{0}\end{array}$ & $\begin{array}{l}0.0^{\mathrm{c}} \\
0.0^{\mathrm{c}} \\
0.0^{\mathrm{c}}\end{array}$ & $\begin{array}{l}0.0^{\mathrm{c}} \\
0.0^{\mathrm{c}} \\
0.0^{\mathrm{c}}\end{array}$ & $\begin{array}{l}0.0^{\mathrm{c}} \\
0.0^{\mathrm{c}} \\
0.0^{\mathrm{c}}\end{array}$ & $\begin{array}{l}9.8650 \times 10^{2} \\
9.8650 \times 10^{2} \\
2.1976 \times 10^{0}\end{array}$ \\
\hline 2 & $\mathrm{C}=\mathrm{C}$ & $\begin{array}{l}a(i, j): \\
b(i, j): \\
c(i, j):\end{array}$ & $\begin{array}{r}2.1450 \times 10^{2} \\
0.0^{\mathrm{b}} \\
0.0^{\mathrm{b}}\end{array}$ & $\begin{array}{r}3.1890 \times 10^{2} \\
0.0^{\mathrm{b}} \\
0.0^{\mathrm{b}}\end{array}$ & $\begin{array}{r}-3.5360 \times 10^{1} \\
0.0^{\mathrm{b}} \\
0.0^{\mathrm{b}}\end{array}$ & $\begin{array}{r}-3.5360 \times 10^{1} \\
0.0^{\mathrm{b}} \\
0.0^{\mathrm{b}}\end{array}$ & $\begin{array}{r}-3.5360 \times 10^{1} \\
0.0^{\mathrm{b}} \\
0.0^{\mathrm{b}}\end{array}$ & $\begin{array}{r}5.2410 \times 10^{2} \\
0.0^{\mathrm{b}} \\
0.0^{\mathrm{b}}\end{array}$ \\
\hline 3 & $\mathrm{ACH}_{\mathrm{n}}$ & $\begin{array}{l}a(i, j): \\
b(i, j): \\
c(i, j):\end{array}$ & $\begin{array}{r}3.2140 \times 10^{1} \\
-1.2963 \times 10^{1} \\
8.0000 \times 10^{-1}\end{array}$ & $\begin{array}{r}5.3740 \times 10^{2} \\
-5.3740 \times 10^{2} \\
-1.4576 \times 10^{-1}\end{array}$ & $\begin{array}{r}-1.1120 \times 10^{1} \\
1.9504 \times 10^{1} \\
8.0000 \times 10^{-1}\end{array}$ & $\begin{array}{r}-1.1120 \times 10^{1} \\
1.9504 \times 10^{1} \\
8.0000 \times 10^{-1}\end{array}$ & $\begin{array}{r}-1.1120 \times 10^{1} \\
1.9504 \times 10^{1} \\
8.0000 \times 10^{-1}\end{array}$ & $\begin{array}{r}6.3610 \times 10^{2} \\
-6.3610 \times 10^{2} \\
2.5444 \times 10^{0}\end{array}$ \\
\hline 7 & $\mathrm{H}_{2} \mathrm{O}$ & $\begin{array}{l}a(i, j): \\
b(i, j): \\
c(i, j):\end{array}$ & $\begin{array}{r}5.4050 \times 10^{2} \\
3.5503 \times 10^{2} \\
-1.8480 \times 10^{0}\end{array}$ & $\begin{array}{r}-6.9290 \times 10^{1} \\
-1.4082 \times 10^{2} \\
-8.0000 \times 10^{-1}\end{array}$ & $\begin{array}{r}1.6230 \times 10^{2} \\
-2.0000 \times 10^{2} \\
-7.9977 \times 10^{-1}\end{array}$ & $\begin{array}{r}3.6210 \times 10^{2} \\
-1.7373 \times 10^{2} \\
--1.4484 \times 10^{0}\end{array}$ & $\begin{array}{r}-8.9710 \times 10^{1} \\
-2.0000 \times 10^{2} \\
-8.0000 \times 10^{-1}\end{array}$ & $\begin{array}{r}-1.5300 \times 10^{2} \\
1.0086 \times 10^{2} \\
-8.0000 \times 10^{-1}\end{array}$ \\
\hline 8 & $\mathrm{ACOH}$ & $\begin{array}{l}a(i, j): \\
b(i, j): \\
c(i, j):\end{array}$ & $\begin{array}{r}-1.6290 \times 10^{2} \\
0.0^{\mathrm{b}} \\
0.0^{\mathrm{b}}\end{array}$ & $\begin{array}{r}4.0890 \times 10^{2} \\
4.0890 \times 10^{2} \\
1.7894 \times 10^{-1}\end{array}$ & $\begin{array}{r}2.7580 \times 10^{2} \\
2.7580 \times 10^{2} \\
9.7679 \times 10^{-1}\end{array}$ & $\begin{array}{r}2.7580 \times 10^{2} \\
2.7580 \times 10^{2} \\
9.7679 \times 10^{-1}\end{array}$ & $\begin{array}{r}2.7580 \times 10^{2} \\
2.7580 \times 10^{2} \\
9.7679 \times 10^{-1}\end{array}$ & $\begin{array}{r}-4.5160 \times 10^{2} \\
4.5160 \times 10^{2} \\
1.8064 \times 10^{-1}\end{array}$ \\
\hline 9 & $\mathrm{CH}_{\mathrm{n}} \mathrm{CO}$ & $\begin{array}{l}a(i, j): \\
b(i, j): \\
c(i, j):\end{array}$ & $\begin{array}{r}-1.0360 \times 10^{2} \\
0.0^{\mathrm{b}} \\
0.0^{\mathrm{b}}\end{array}$ & $\begin{array}{r}6.6940 \times 10^{2} \\
-6.6935 \times 10^{2} \\
-2.6776 \times 10^{0}\end{array}$ & $\begin{array}{r}2.6760 \times 10^{1} \\
-50597 \times 10^{1} \\
-8.0000 \times 10^{-1}\end{array}$ & $\begin{array}{r}2.6760 \times 10^{1} \\
-5.0597 \times 10^{1} \\
-8.0000 \times 10^{-1}\end{array}$ & $\begin{array}{r}2.6760 \times 10^{1} \\
-50597 \times 10^{1} \\
-8.0000 \times 10^{-1}\end{array}$ & $\begin{array}{r}1.6450 \times 10^{2} \\
2.0000 \times 10^{2} \\
8.0000 \times 10^{-1}\end{array}$ \\
\hline 10 & $\mathrm{CHO}$ [aldehyde] & $\begin{array}{l}a(i, j): \\
b(i, j): \\
c(i, j):\end{array}$ & $\begin{array}{r}3.0410 \times 10^{2} \\
0.0^{\mathrm{b}} \\
0.0^{\mathrm{b}}\end{array}$ & $\begin{array}{r}4.9750 \times 10^{2} \\
-4.9750 \times 10^{2} \\
1.9900 \times 10^{0}\end{array}$ & $\begin{aligned} 5.0570 & \times 10^{2} \\
5.0569 & \times 10^{2} \\
-2.0228 & \times 10^{0}\end{aligned}$ & $\begin{aligned} 5.0570 & \times 10^{2} \\
5.0569 & \times 10^{2} \\
-2.0228 & \times 10^{0}\end{aligned}$ & $\begin{aligned} 5.0570 \times 10^{2} \\
5.0569 \times 10^{2} \\
-2.0228 \times 10^{0}\end{aligned}$ & $\begin{array}{r}5.2900 \times 10^{2} \\
-5.2900 \times 10^{2} \\
-2.1160 \times 10^{0}\end{array}$ \\
\hline 11 & $\mathrm{CCOO}$ & $\begin{array}{l}a(i, j): \\
b(i, j): \\
c(i, j):\end{array}$ & $\begin{array}{r}-2.3570 \times 10^{2} \\
-2.1770 \times 10^{2} \\
-9.4280 \times 10^{-1}\end{array}$ & $\begin{array}{r}6.6020 \times 10^{2} \\
-4.2917 \times 10^{2} \\
0.0^{b}\end{array}$ & $\begin{array}{r}1.1480 \times 10^{2} \\
2.0000 \times 10^{2} \\
8.0000 \times 10^{-1}\end{array}$ & $\begin{array}{r}1.1480 \times 10^{2} \\
2.0000 \times 10^{2} \\
8.0000 \times 10^{-1}\end{array}$ & $\begin{array}{r}1.1480 \times 10^{2} \\
2.0000 \times 10^{2} \\
8.0000 \times 10^{-1}\end{array}$ & $\begin{array}{r}2.4540 \times 10^{2} \\
2.4540 \times 10^{2} \\
9.8160 \times 10^{-1}\end{array}$ \\
\hline 13 & $\mathrm{CH}_{\mathrm{n}} \mathrm{O}$ [ether] & $\begin{array}{l}a(i, j): \\
b(i, j): \\
c(i, j):\end{array}$ & $\begin{array}{r}0.0 \\
0.0 \\
0.0\end{array}$ & $\begin{array}{r}6.6460 \times 10^{2} \\
-6.6460 \times 10^{2} \\
2.6560 \times 10^{0}\end{array}$ & $\begin{array}{r}8.3360 \times 10^{1} \\
2.0000 \times 10^{2} \\
8.0000 \times 10^{-1}\end{array}$ & $\begin{array}{r}8.3360 \times 10^{1} \\
2.0000 \times 10^{2} \\
8.0000 \times 10^{-1}\end{array}$ & $\begin{array}{r}8.3360 \times 10^{1} \\
2.0000 \times 10^{2} \\
8.0000 \times 10^{-1}\end{array}$ & $\begin{array}{r}2.3770 \times 10^{2} \\
2.3770 \times 10^{2} \\
9.5080 \times 10^{-1}\end{array}$ \\
\hline 65 & $\mathrm{COOH}$ & $\begin{array}{l}a(i, j): \\
b(i, j): \\
c(i, j):\end{array}$ & $\begin{array}{l}-3.3850 \times 10^{2} \\
-1.4574 \times 10^{2} \\
3.1930 \times 10^{-1}\end{array}$ & $\begin{array}{l}0.0 \\
0.0 \\
0.0\end{array}$ & $\begin{array}{l}3.1530 \times 10^{2} \\
3.1530 \times 10^{2} \\
1.2612 \times 10^{0}\end{array}$ & $\begin{array}{l}3.1530 \times 10^{2} \\
3.1530 \times 10^{2} \\
1.2612 \times 10^{0}\end{array}$ & $\begin{array}{l}3.1530 \times 10^{2} \\
3.1530 \times 10^{2} \\
1.2612 \times 10^{0}\end{array}$ & $\begin{array}{r}-1.0303 \times 10^{2} \\
2.0000 \times 10^{2} \\
8.0000 \times 10^{-1}\end{array}$ \\
\hline 66 & $\mathrm{CH}_{\mathrm{n}}^{[\text {alc }]}$ & $\begin{array}{l}a(i, j): \\
b(i, j): \\
c(i, j):\end{array}$ & $\begin{array}{r}2.5150 \times 10^{2} \\
-1.7943 \times 10^{2} \\
5.5434 \times 10^{-1}\end{array}$ & $\begin{array}{l}6.6350 \times 10^{2} \\
6.6350 \times 10^{2} \\
2.6540 \times 10^{0}\end{array}$ & $\begin{array}{l}0.0 \\
0.0 \\
0.0\end{array}$ & $\begin{array}{l}0.0^{\mathrm{c}} \\
0.0^{\mathrm{c}} \\
0.0^{\mathrm{c}}\end{array}$ & $\begin{array}{l}0.0^{\mathrm{c}} \\
0.0^{\mathrm{c}} \\
0.0^{\mathrm{c}}\end{array}$ & $\begin{array}{l}9.8650 \times 10^{2} \\
9.8650 \times 10^{2} \\
2.1976 \times 10^{0}\end{array}$ \\
\hline 67 & $\mathrm{CH}_{\mathrm{n}}^{[\text {[alc-tail }]}$ & $\begin{array}{l}a(i, j): \\
b(i, j): \\
c(i, j):\end{array}$ & $\begin{array}{r}2.5150 \times 10^{2} \\
-1.7943 \times 10^{2} \\
5.5434 \times 10^{-1}\end{array}$ & $\begin{array}{l}6.6350 \times 10^{2} \\
6.6350 \times 10^{2} \\
2.6540 \times 10^{0}\end{array}$ & $\begin{array}{l}0.0^{\mathrm{c}} \\
0.0^{\mathrm{c}} \\
0.0^{\mathrm{c}}\end{array}$ & $\begin{array}{l}0.0 \\
0.0 \\
0.0\end{array}$ & $\begin{array}{l}0.0^{\mathrm{c}} \\
0.0^{\mathrm{c}} \\
0.0^{\mathrm{c}}\end{array}$ & $\begin{array}{l}9.8650 \times 10^{2} \\
9.8650 \times 10^{2} \\
2.1976 \times 10^{0}\end{array}$ \\
\hline 68 & $\mathrm{CH}_{\mathrm{n}}^{[\mathrm{OH}]}$ & $\begin{array}{l}a(i, j): \\
b(i, j): \\
c(i, j):\end{array}$ & $\begin{array}{r}2.5150 \times 10^{2} \\
-1.7943 \times 10^{2} \\
5.5434 \times 10^{-1}\end{array}$ & $\begin{array}{l}6.6350 \times 10^{2} \\
6.6350 \times 10^{2} \\
2.6540 \times 10^{0}\end{array}$ & $\begin{array}{l}0.0^{\mathrm{c}} \\
0.0^{\mathrm{c}} \\
0.0^{\mathrm{c}}\end{array}$ & $\begin{array}{l}0.0^{\mathrm{c}} \\
0.0^{\mathrm{c}} \\
0.0^{\mathrm{c}}\end{array}$ & $\begin{array}{l}0.0 \\
0.0 \\
0.0\end{array}$ & $\begin{array}{l}9.8650 \times 10^{2} \\
9.8650 \times 10^{2} \\
2.1976 \times 10^{0}\end{array}$ \\
\hline 69 & $\mathrm{OH}$ & $\begin{array}{l}a(i, j): \\
b(i, j): \\
c(i, j):\end{array}$ & $\begin{array}{r}2.8060 \times 10^{1} \\
-1.2899 \times 10^{2} \\
-8.0000 \times 10^{-1}\end{array}$ & $\begin{array}{r}2.2439 \times 10^{2} \\
2.2439 \times 10^{2} \\
8.9756 \times 10^{-1}\end{array}$ & $\begin{array}{r}1.5640 \times 10^{2} \\
2.0000 \times 10^{2} \\
-8.0000 \times 10^{-1}\end{array}$ & $\begin{array}{r}1.5640 \times 10^{2} \\
2.0000 \times 10^{2} \\
-8.0000 \times 10^{-1}\end{array}$ & $\begin{array}{r}1.5640 \times 10^{2} \\
2.0000 \times 10^{2} \\
-8.0000 \times 10^{-1}\end{array}$ & $\begin{array}{l}0.0 \\
0.0 \\
0.0\end{array}$ \\
\hline
\end{tabular}

a The values of $a_{i, j}$ for $\mathrm{OH}, \mathrm{CH}_{\mathrm{n}}^{[\text {alc] }}, \mathrm{CH}_{\mathrm{n}}^{[\text {alc-tail] }}, \mathrm{CH}_{\mathrm{n}}^{[\mathrm{OH}]}$ interactions with $\mathrm{H}_{2} \mathrm{O}$ are taken from Marcolli and Peter (2005). The $a_{i, j}$ values for $\mathrm{COOH} \leftrightarrow \mathrm{H}_{2} \mathrm{O}$ group interactions are taken from Peng et al. (2001). For all other functional groups the $a_{i, j}$ values from the revised parameter set of Hansen et al. (1991) are used.

${ }^{\mathrm{b}}$ Main group interaction parameters $b_{i, j}$ and $c_{i, j}$ are set to zero since appropriate data to determine these interactions are missing.

${ }^{\mathrm{c}}$ Interaction parameters between different types of alkyl main groups $\left(\mathrm{CH}_{\mathrm{n}}, \mathrm{CH}_{\mathrm{n}}^{[\mathrm{alc}]}, \mathrm{CH}_{\mathrm{n}}^{[\text {alc-tail] }}\right.$, and $\left.\mathrm{CH}_{\mathrm{n}}^{[\mathrm{OH}]}\right)$ are set to zero. 
when there is a solid-solid phase transition (change of polymorphic form) between $T$ and $T_{\mathrm{m}, i}$. Note that if more than one solid-solid phase transition is present in the temperature range of interest, additional terms (of the form of the second term) need to be added in Eq. (19) to account for each of these phase changes. Equation (19) uses the simplification that the melting temperature and the triple point temperature of an organic compound are relatively close at atmospheric pressure. For obtaining activity coefficients from experimental data at given temperatures and mole fractions $\left(x_{\text {org }}, T\right)$, Eq. (19) can be solved for the SLE organic activity and/or activity coefficients. Pure component physicochemical properties such as $\Delta h_{\mathrm{m}, i}$ and $\Delta c_{p, \mathrm{~m}, i}$ are obtained from tabulated experimental data (Domalski and Hearing, 1996; Dean, 1999). We note that for certain organic compounds rather large uncertainties in the physicochemical property values used in Eq. (19) will translate into large uncertainties in the calculated SLE activity values, particularly when the target temperature is far from the melting point temperature at standard pressure. In this work, such uncertainties were assessed based on the comparison of derived activity values with activity values from other data types for the same system, and by means of preliminary model fits of AIOMFAC-P3. Affected data sets were either assigned a much lower weighting or zero weighting (removing the data set from the fit).

\subsection{Water activity measurements}

Water activity measurements were conducted for aqueous organic solutions with an Aqualab dew point water activity meter (Model 3TE, Decagon Devices, USA), which enables water activity measurements within the temperature range from $289-313 \mathrm{~K}$ for several concentrations at each temperature level. Water activity data for measured binary aqueous organic bulk solutions are tabulated in Tables A1-A8. Additional measurements of aqueous multi-functional organic solutions are provided in Ganbavale et al. (2014). Measured water activities were then used directly for the AIOMFAC-P3 parameter determination - with the exception of data within $\pm 10 \mathrm{~K}$ from $298 \mathrm{~K}$.

\subsection{Liquid-liquid equilibria data}

The equilibrium state between coexisting liquid phases is known as liquid-liquid equilibrium (LLE). Liquid-liquid equilibria are useful as a source of data for systems containing relatively hydrophobic organic compounds and water, with a miscibility gap that depends on temperature and mixture composition. In general, multi-component systems may form more than two phases (in binary systems at maximum two liquid phases may coexist). For salt-free aqueous organic systems with two coexisting liquid phases, usually one phase is an aqueous (water-rich) phase while the other is an organic-rich phase. Most available experimental LLE data has been measured relatively close to room temperature and is useful for a better description of the phase behaviour. However, for the purpose of our new parameterisation of AIOMFAC with regard to low temperatures far from room temperature, the LLE data tend to be less useful than, e.g. SLE data. We use the tie-line data from LLE measurements, which represents the composition of the two liquid phases in equilibrium at a certain temperature. A direct calculation and comparison of activities in coexisting phases is possible at experimental LLE compositions, i.e. measured mole fractions $x_{j}^{\alpha}$ and $x_{j}^{\beta}$ of the two liquid phases $\alpha$ and $\beta$ at equilibrium. According to the reference state definitions of AIOMFAC, different independent components $j$ should have the same activities in coexisting phases, i.e. $a_{j}^{(x), \alpha}=a_{j}^{(x), \beta}$. This data type can therefore be implemented in the model fit by minimising the relative differences between the activities of the components in the two liquid phases. We use the method introduced by Zuend et al. (2011) for the comparison of calculated relative activity deviations between the activities of components $j$ present in the two phases.

Furthermore, we also performed AIOMFAC-based predictions of the phase compositions at LLE using the method of Zuend and Seinfeld (2013), particularly for the graphical comparison of measured and predicted tie-line LLE data. To perform such predictions, an initial composition point is required from which a liquid-liquid equilibrium calculation is performed in order to determine whether the initial mixture composition is stable as a single homogeneous phase or whether two coexisting liquid phases represent the stable equilibrium state (according to the model) and what the compositions of the two phases are in the LLE case. An initial mixture composition with mole fraction $x_{j}^{\text {init }}$ of component $j$ on a unstable / metastable point of an experimental tie-line can be generated by the following:

$x_{j}^{\mathrm{init}}=\frac{1}{2}\left(x_{j}^{\alpha}+x_{j}^{\beta}\right)$.

Such LLE predictions from an initial composition are computationally more expensive than the relative activity difference calculations used in the model fit, yet offer a different view on the performance of the model for applications of phase separation / phase composition computations. For more details about the LLE computations with AIOMFAC we refer to Zuend et al. (2010) and Zuend and Seinfeld (2013); the specific method used for fitting LLE data based on relative activity deviations is described in more detail in Zuend et al. (2011).

\subsection{Vapour-liquid equilibria}

VLE data represent the temperature and pressure conditions where a liquid (mixture) and its vapour(s) (gas phase) are in equilibrium with each other. The VLE data are usually obtained by performing measurements either at isobaric or isothermal conditions. VLE data considered in the model include binary water + organic systems and binary 


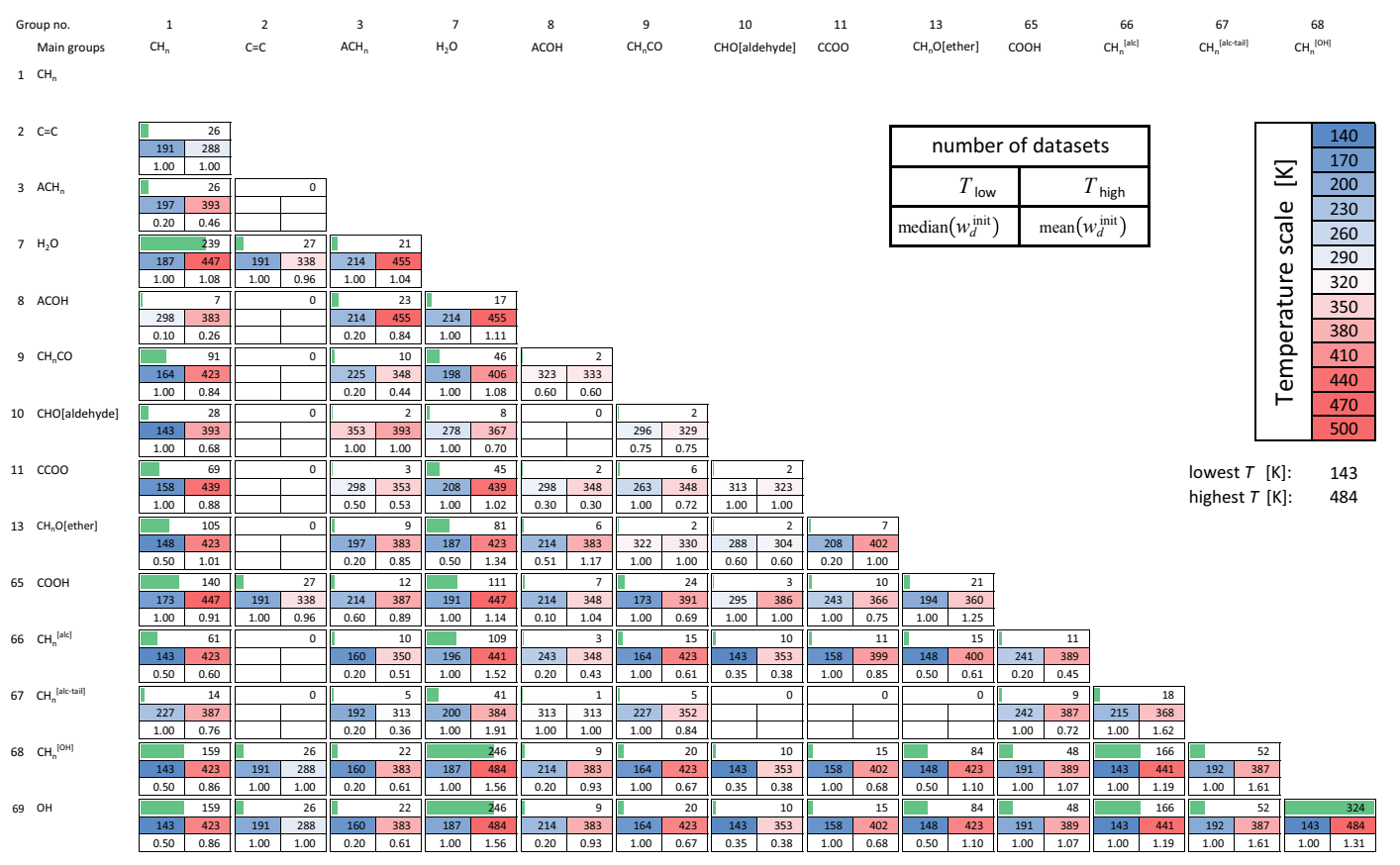

Figure 1. Database distribution for the water $\leftrightarrow$ organic and organic $\leftrightarrow$ organic interaction parameters. The table lists for each main group interaction pair at temperatures substantially different from the reference temperature, $T_{\ominus}=298.15 \mathrm{~K}$, i.e. per data set $d$ : $T_{d}$, low $<289 \mathrm{~K}$ or $T_{d, \text { high }}>307 \mathrm{~K}$, the following information. Top boxes: the total number of data sets available, visualised by the green bars. Middle boxes: the lowest temperature $\left(T_{\text {low }}\right.$ ) and the highest temperature $\left(T_{\text {high }}\right)$ (units of K) of the data points using a percentile-wise colour scale. Bottom boxes: the median and arithmetic mean of the assigned initial data set weighting values $\left(w_{d}^{\text {init }}\right)$ of the data sets involved.

data for water-free organic (1) + organic (2) systems. Since isobaric measurements are usually conducted at $1 \mathrm{~atm}(=$ $101.325 \mathrm{kPa}$ ) pressure by measuring the boiling point temperature, they typically provide data at relatively high temperatures. In order to be used for the model parameterisation, the composition of the liquid in terms of mole fraction $x_{j}$ of each component $j$, the composition of the gas phase in terms of mole fraction $y_{j}$ and the total pressure $p$ of the gas phase have to be stated or need to be derived from the data source. VLE data provide the composition dependence of activity coefficients. Assuming that the gas phase can be treated as an ideal gas mixture, activity coefficients of the components in the solution can be calculated by modified Raoult's law:

$\gamma_{j}^{(x)}=\frac{p_{j}}{p_{j}^{\circ}(T) x_{j}}, \quad p_{j}=y_{j} p$.

Here, $p_{j}$ is the partial pressure of component $j$, and $p_{j}^{\circ}(T)$ is the pure liquid component saturation vapour pressure calculated at the measurement temperatures using the Antoine equation with coefficients from the Landolt-Börnstein database (Dykyj et al., 2000), from Yaws et al. (2005) or, in some cases, the $p_{j}^{\circ}(T)$ are directly available from the reference of the experimental VLE data. Except for monocarboxylic acids such as formic, acetic, and propionic acid, which exhibit significant gas phase association (dimers, trimers), assuming an ideal gas mixture for the total pressure and temperature ranges of the data is acceptable. Other ex- ceptions include certain diols and triols, e.g. glycerol, which show moderate non-ideality in the gas phase, requiring fugacity corrections. For mono-alcohols, fugacity corrections of the gas phase did not lead to substantial changes in activity coefficients, due to the form of Eq. (21) (where the ratio of partial pressure and saturation vapour pressure, both similarly affected by association effects, cancel most of the non-ideality effects), and were typically ignored. For glycerol, fugacity corrections were not applied; instead, given the large amount of data sets covering functional groups of alcohols, the glycerol VLE data sets were assigned a very small weighting, essentially excluding their influence on the parameter fit. To account for the gas-phase dimerisation of carboxylic acids we obtain the monomer partial pressures using the dimerisation equilibrium coefficients from Tsonopoulos and Prausnitz (1970). The procedure for calculating experimental activity coefficients using this dimerization correction is described in more detail in Zuend et al. (2011).

\section{Objective function and model parameter estimation}

Organic-organic and organic-water main group interactions are parameterised in the SR part of AIOMFAC. The model parameter determination procedure involves simultaneous fitting of the various group interaction parameters to available thermodynamic phase equilibria data (see the database 
overview in Fig. 1). In order to ensure inter-comparability of different thermodynamic quantities and with due consideration of the various aspects of uncertainty in measurements and the group-contribution concept of the model, we use the following general objective function $\left(F_{\mathrm{obj}}\right)$, subject to minimisation (Zuend et al., 2011):

$$
F_{\mathrm{obj}}=\sum_{d} \sum_{u} w_{d, u}\left[\frac{Q_{d, u}^{\mathrm{calc}}-Q_{d, u}^{\mathrm{ref}}}{\left|Q_{d, u}^{\mathrm{ref}}\right|+Q_{d, u}^{\mathrm{tol}}}\right]^{2}
$$

Here, $w_{d, u}$ is the weighting value of a data point and the sums cover all data points $u$ in all data sets $d$ considered. $Q_{d, u}^{\text {ref }}$ is a reference quantity, directly determined from experiments (e.g. a measured water activity value at a certain $T$ and $x_{\mathrm{w}}$ ) or derived from measurements by means of thermodynamic relations, e.g. SLE water activity on the ice melting curve at a specific temperature. $Q_{d, u}^{\text {calc }}$ represents the corresponding quantity calculated with the model at the given conditions. $Q_{d, u}^{\text {tol }}$ is a tolerance quantity ( $>0$ ) which represents the measurement uncertainty or model sensitivity and has the same units as $Q_{d, u}^{\text {ref }}$. During the iterative fitting of the model parameters, we use the AIOMFAC model (with the so far fitted parameter set at that iteration step) to calculate the model activity sensitivity with respect to an assumed representative uncertainty in absolute mixture composition, a mole fraction tolerance set to $x^{\text {tol }}=0.01$. We refer to Zuend et al. (2011) (their Sect. 3.3) for a detailed description of how the model sensitivity is calculated. We use the AIOMFAC model to calculate the effect of a tiny change in composition on the activity coefficients of the different mixture components by means of a total molar derivative. Technically, this is done by scaling and summation of the partial derivatives of the activity coefficients at a given solution composition by means of finite differences in molar composition (Eq. 10 of Zuend et al., 2011).

\subsection{Data set weighting and temperature range}

Both experimental uncertainties and model deficiencies need to be considered while determining the main group interaction parameters. The measured experimental quantities have some level of random and systematic errors, which may also depend on mixture composition, rendering some data points more reliable than others. This is considered during the parameter estimation procedure by giving appropriate weighting to the data sets and by data point-specific tolerance quantities computed in parallel from the model sensitivities as the iterative model fit progresses. With the aim of reducing a disproportionate influence of data sets with a large number of data points, as well as preventing an immoderate high weighting of data sets with a small number of data points, Zuend et al. (2011) propose a simplified procedure of assigning individual weighting to data sets on the basis of data type and number of data points $N_{d}$ in a data set:

$w_{d, u}= \begin{cases}w_{d}^{\text {init }} & \text { if } N_{d} \leq \eta \\ w_{d}^{\text {init }} \times \frac{\eta}{N_{d}} & \text { if } N_{d}>\eta,\end{cases}$

where $w_{d}^{\text {init }}$ is an initial weighting of data set $d$ on the basis of its temperature range, data type, and, in certain cases, additional expert judgement of its reliability. $\eta$ is a characteristic number of data points per data set. The weighting of individual data points that are part of large data sets can be reduced by multiplication with $\eta / N_{d}$. In this work, we keep $\eta=10$ as in Zuend et al. (2011). Initial weightings assigned to data sets for the model fit are given in Table 1.

With the goal of fitting the AIOMFAC model parameters for a better description of activities at (low/high) temperatures far from room temperature, a set of rules was applied to assign initial weightings based on data type and the temperature range covered. Low temperature $a_{\mathrm{w}}$ data were assigned an initial weighting $w_{d}^{\text {init }}=5.0$ while the SLE organic activity (SLE(org)) data sets (i.e. SLE data where an organic compound forms the solid in equilibrium with the liquid solution) are given an initial weighting of $w_{d}^{\text {init }}=0.2$ because of the lower reliability of deriving solute activities using Eq. (19) compared to calculating water activities with Eq. (17). Relying on the water activity parameterisation of homogeneous freezing temperatures in aqueous solutions (Koop and Zobrist, 2009), freezing point depressions were also used as data source for parameter fitting. The $a_{\mathrm{w}}$ from differential scanning calorimetry (DSC) measurements at homogeneous freezing temperatures $\left(T_{\mathrm{hom}}\right)$ are assigned $w_{d}^{\text {init }}=1.0$ (considering some uncertainties associated with the $T_{\text {hom }}$ determination from DSC measurements). The weighting of all types of data sets close to room temperature (289-307 K) are set to zero to keep AIOMFAC unchanged around room temperature and guarantee consistency with functional groups that were not included in the new three-parameter temperature-dependence parameterisation. The LLE and VLE data sets are assigned an initial weighting of $w_{d}^{\text {init }}=1.0$. However, data sets showing large scatter or inconsistencies with other comparable data (direct comparison of measurements or comparable via the thermodynamic relations underpinning AIOMFAC) were given lower or even zero weightings. To obtain parameters representing the best simultaneous description of all phase equilibria, thermodynamically inconsistent data have been excluded from the parameter fitting process (but only after test runs and a careful data quality review).

For determining the set of main group interaction parameters, i.e. the set of $b_{m, n}$ and $c_{m, n}$ values, where $m, n$ represent all combinations of different main groups, we use a set of selective criteria by considering the temperature range of available experimental data. These criteria are separately applied to each group interaction pair as follows: the $b_{m, n}$ values are determined only if: $\Delta T_{\text {low }}=\left|T_{\text {low }}-T_{\ominus}\right|$ or $\Delta T_{\text {high }}=\left|T_{\text {high }}-T_{\ominus}\right|>40 \mathrm{~K}$ and $\Delta T=\left|T_{\text {low }}-T_{\text {high }}\right|>$ 
$40 \mathrm{~K}$, where $T_{\text {low }}$ and $T_{\text {high }}$ are the lowest and highest temperatures covered by the data points involved (see Fig. 1) and $T_{\ominus}=298.15 \mathrm{~K}$ is the reference temperature. Similarly, the $c_{m, n}$ parameters for the main groups are determined only if $\Delta T_{\text {low }}>80 \mathrm{~K}$ or $\Delta T_{\text {high }}>80 \mathrm{~K}$ and if $\Delta T>80 \mathrm{~K}$. In addition, we set numeric bounds on the values of the fitted parameters, described below. The three terms on the right hand side of Eq. (16) contain parameters of different thermodynamical meaning (see Eq. 15) and different magnitude. The terms containing $a_{m, n}$ and $b_{m, n}$ are associated with changes of molar enthalpy over a certain temperature difference, while $c_{m, n}$ is related to changes in the molar heat capacity at constant pressure (hence, accounting for the change of the change of enthalpy with temperature). These thermodynamic quantities tend to be of different magnitude. In the temperature range of interest here, molar heat capacity changes (units of $\mathrm{J} \mathrm{mol}^{-1} \mathrm{~K}^{-1}$ ) are roughly 2 to 3 orders of magnitude smaller in value than changes in molar enthalpy (units of $\mathrm{J} \mathrm{mol}^{-1}$ ). Hence, the expected values and set numeric bounds on the parameters $b_{m, n}$ and $c_{m, n}$ are quite different for these reasons. Symmetric parameter bounds for permissible values of $b_{m, n}$ are set to $\pm \max \left[a_{m, n}, 200\right]$, while the numerical limits on $c_{m, n}$ are set to $\pm \max \left[4 \times 10^{-3} \times \max \left(a_{m, n}, 200\right)\right]$.

With the implementation of these parameter bounds and based on the reduced set of experimental data fulfilling the selection criteria, 150 new short-range interaction parameters were determined simultaneously for 14 functional main groups. As described in Zuend et al. (2011), due to the high dimensionality, and nonlinear coupling of the fit parameters, the minimisation problem is a challenging task for any global optimisation method. For the parameter optimisation, it is sufficient to find a "good" local minimum, which may not be the global minimum. As a part of data quality control and to avoid that a few data sets dominate the parameter optimisation due to potential numerical issues or other reasons, such as inconsistent data sets and outliers, a large number of trial parameter optimisation runs were carried out. To solve the parameter optimisation problem, we use the formulation introduced by Zuend et al. (2011) by using a combination of algorithms to solve the parameter optimisation problem. First, a best-of-random differential evolution (BoRDE) algorithm (Lin et al., 2011) is used to explore the parameter space and to broadly locate a minimum of $F_{\mathrm{obj}}$. Second, the global trust region method BOBYQA (Bound Optimization BY Quadratic Approximation) of Powell (2009) is applied to further refine the solution. Finally, the downhill simplex algorithm by Nelder and Mead (1965) is used to fully converge to the minimum. More details are given in Zuend et al. (2011).

During trial runs, the contributions of the individual data sets to the objective function value (Eq. 22) were used to identify potential inconsistencies among data sets, errors in data calculations and conversion or the implementation in the model. This allowed us to establish a high level of data quality, correct mistakes (e.g. typing errors) and compare thermo- dynamic data from different types of experiments and references for general consistency. Table 2 provides the final values of the determined organic main group interaction parameters. For comparison and completeness, the values of $a_{m, n}$ parameters, which were preserved in the new AIOMFAC parameterisation are listed as well. All main group interaction parameters $b_{m, n}$ and $c_{m, n}$, for which the database does not satisfy our criteria concerning temperature range and data availability, are set to zero.

\section{Results and discussion}

The new temperature dependence parameterisation is applied to aqueous organic and water-free organic solutions covering a wide concentration and temperature range. As discussed in Sect. 4.1, the database and therefore the values of $F_{\text {obj }}$ (Eq. 22) evaluated with both AIOMFAC-P3 and AIOMFAC-P1 for comparison, do not include data sets with data points exclusively near room temperature $(298 \pm 10 \mathrm{~K})$. In this section, we compare the model performance of the new AIOMFAC-P3 version, with AIOMFAC-P1 (original AIOMFAC version) based on overall quantitative measures followed by a discussion of a selection of aqueous organic mixtures and water-free organic mixtures.

The new AIOMFAC-P3 parameterisation for the temperature dependence of activity coefficients shows an overall improvement of $28 \%$ in terms of $F_{\text {obj }}$ in comparison to AIOMFAC-P1 (542 data sets involved). As stated earlier, AIOMFAC-P1 uses the temperature-dependence expression of standard UNIFAC and represents the AIOMFAC performance using only $a_{m, n}$ interaction parameters. The AIOMFAC-P3 model version uses all the three parameters i.e. $a_{m, n}, b_{m, n}$ and $c_{m, n}$, where applicable, with our new expression for the temperature dependence of group interactions.

For the purpose of evaluating the improvement of the new parameterisation it is of interest to compare the performance of the two AIOMFAC model versions for different subsets of the database covering separately low and high temperature ranges and certain aspects of the complexity of molecular structures involved ("monofunctional" vs. "multi-functional" organic components). We define the value $F_{\text {obj, low- } T \text { calcu- }}$ lated as the objective function value based on Eq. (22) when exclusively considering data sets with $T_{\text {high }}<274 \mathrm{~K}$. That is, the subset of the database including only data sets containing data points with a maximum temperature below $274 \mathrm{~K}$ (at least $25 \mathrm{~K}$ below the reference temperature of $298 \mathrm{~K}$ ). This serves to represent the low-temperature range in our comparison. Analogously, to represent the high-temperature range (at least $25 \mathrm{~K}$ above $298 \mathrm{~K}$ ), we define $F_{\text {obj, high- } T}$ by exclusively considering the data sets with $T_{\text {low }}>322 \mathrm{~K}$. The minimum distance of $25 \mathrm{~K}$ from the reference temperature was chosen such that there is (i) a clear difference between the low and high temperature ranges considered, yet that (ii) still 

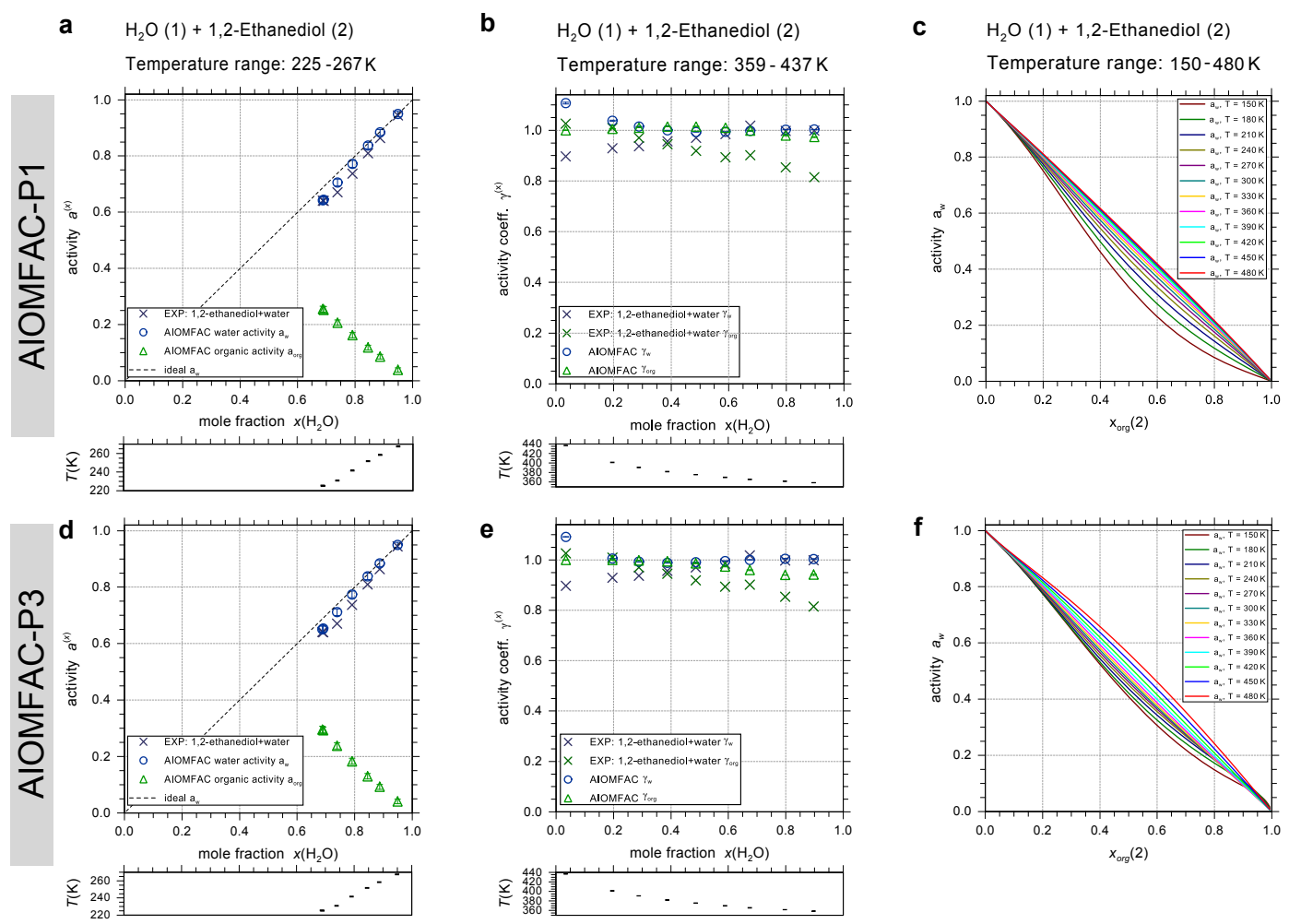

Figure 2. SLE and VLE measurements for water (1) + 1,2-ethanediol (2) solutions and corresponding calculations of AIOMFAC-P1 (a-c) or AIOMFAC-P3 (d-f). The coloured curves in (c, f) represent the temperature dependence of water activities predicted for the range 150-480 K. (a, d) Low temperature experimental SLE data (crosses) are compared with the predictions for water activity at the same compositions and temperatures (blue circles). Predictions of the corresponding organic activities are shown as well (green triangles). The dashed line represents the hypothetical water activity of an ideal mixture. The error bars represent the model sensitivity to a composition variation by $x^{\text {tol }}=0.01$. Panels (b, e) show the model predictions of the activity coefficients compared to VLE data covering temperatures significantly higher than room temperature. The temperatures of the individual data points are given in the boxes below the main panels. Experimental data: Ott et al. (1972) and Gmehling and Onken (2003a).

many data sets are included in the comparison (especially given that low-temperature SLE-derived water activity data usually starts at the melting point temperature of pure waterice). Based on this distinction into low and high temperature ranges and from the evaluation of $F_{\mathrm{obj}}$, low- $T$ and $F_{\mathrm{obj}}$, high- $T$ with both AIOMFAC versions, it is found that AIOMFAC$\mathrm{P} 3$ improves similarly in both temperature ranges relative to AIOMFAC-P1. For the low-temperature range the improvement of AIOMFAC-P3 is $37 \%$ (152 data sets involved; $F_{\text {obj, low- }}($ AIOMFAC-P3 $\left.)=10.207\right)$, while for the hightemperature range the improvement is $37 \%$ (223 data sets involved; $F_{\mathrm{obj}}$, high- $T$ (AIOMFAC-P3) $\left.=37.554\right)$. The better improvement in the lower and higher temperature ranges compared to the overall improvement (of $28 \%$ ) is not surprising. This is simply because the two additional fit parameters in AIOMFAC-P3 have a relatively small effect on the model performance in the $\pm 25 \mathrm{~K}$ range around the reference temperature. Therefore, the AIOMFAC-P3 improvement relative to AIOMFAC-P1 is better when the data sets covering the temperature range close to room temperature are excluded from the comparison (to clarify: these data are not excluded from the AIOMFAC-P3 fit - except for data sets within the $10 \mathrm{~K}$ margin around $298 \mathrm{~K}$, which are also not considered in the overall model performance comparison).

We further differentiate the low and high temperature subsets of the database each into two classes of (i) data sets containing monofunctional organic compounds only and (ii) data sets containing at least one multi-functional organic compound. The terminology applied here is to call an organic compound "monofunctional" when its molecular structure contains only one oxygen-bearing subgroup (e.g. phenol, 2-butanol, or palmitic acid), while glycerol, sucrose, 2-ethoxyethanol, glutaric acid, vanillylmandelic acid, and resorcinol are examples for multi-functional compounds included in our database (see Table 1). Despite this terminology, in AIOMFAC/UNIFAC the compounds termed monofunctional here are typically also composed of several types of subgroups (e.g. different $\mathrm{CH}_{n}$ and $\mathrm{ACH}_{n}$ groups in addition to an oxygen-bearing subgroup). Multifunctional oxygenated compounds are often found as major contributors to the total mass of the organic aerosol fraction (e.g. Hallquist et al., 2009). The results from 
a $\quad \mathrm{H}_{2} \mathrm{O}(1)+$ Acetic acid (2) Temperature range: $249-272 \mathrm{~K}$
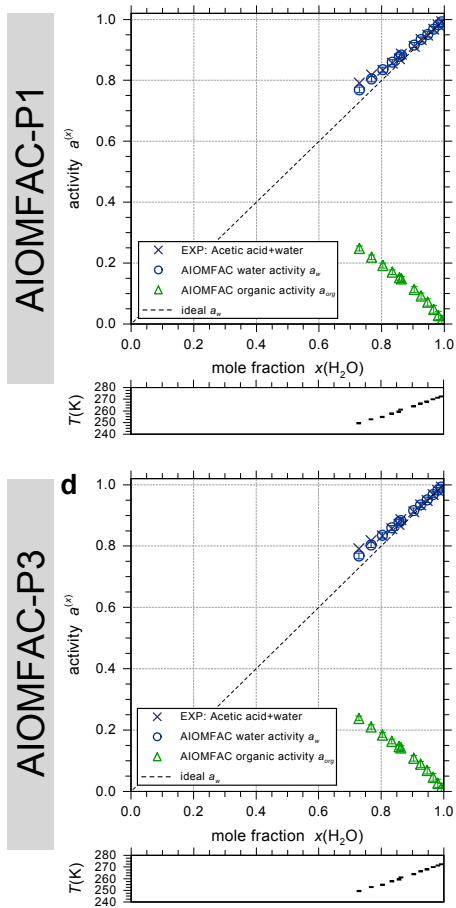

b

$\mathrm{H}_{2} \mathrm{O}(1)+$ Acetic acid (2) Temperature range: $373-386 \mathrm{~K}$
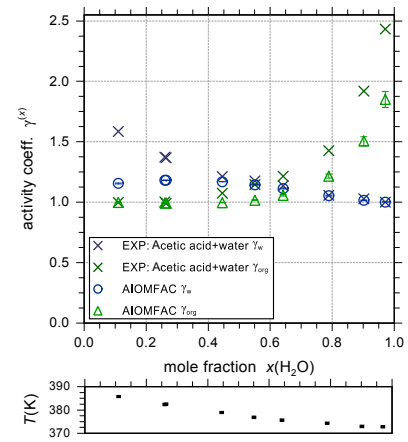

e

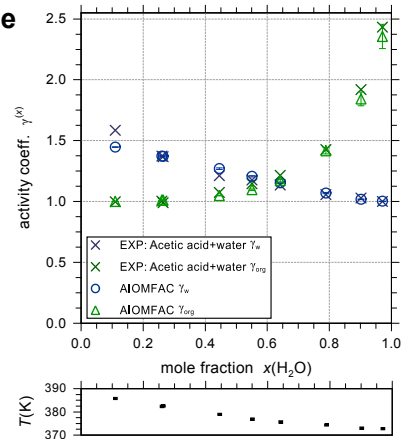

$\mathrm{H}_{2} \mathrm{O}(1)+$ Acetic acid (2)

Temperature range: $150-480 \mathrm{~K}$

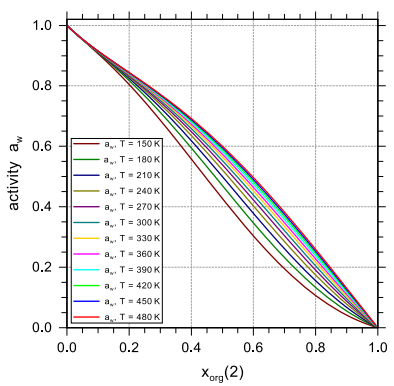

f

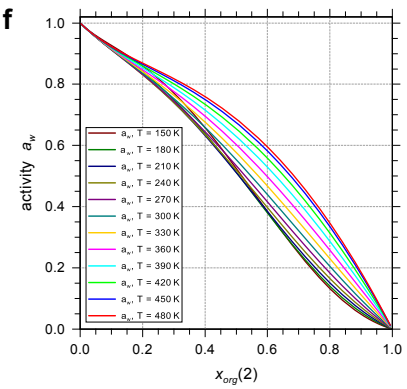

Figure 3. SLE and VLE measurements for water (1) + acetic acid (2) solutions and corresponding calculations of AIOMFAC-P1 (a-c) or AIOMFAC-P3 (d-f). The coloured curves in (c,f) represent the temperature dependence of water activities predicted for the range 150-480 K. (a, d) Low temperature experimental SLE data (crosses) are compared with the predictions for water activity at the same compositions and temperatures (blue circles). Predictions of the corresponding organic activities are shown as well (green triangles). The dashed line represents the hypothetical water activity of an ideal mixture. The error bars represent the model sensitivity to a composition variation by $x^{\text {tol }}=0.01$. Panels $(\mathbf{b}, \mathbf{e})$ show the model predictions of the activity coefficients compared to VLE data covering temperatures significantly higher than room temperature. The temperatures of the individual data points are given in the boxes below the main panels. Experimental data: Faucon (1910) and Narayana et al. (1985).

the evaluation with these subclasses in terms of average improvement of AIOMFAC-P3 compared to AIOMFACP1 for the low-temperature range are as follows: $55 \%$ (46 data sets; $F_{\text {obj, low- } T \text {, mono }}($ AIOMFAC-P3) $=5.197)$ for the subset of data sets with monofunctional compounds and $-7 \%\left(106\right.$ data sets; $F_{\text {obj, low- } T \text {, multi }}($ AIOMFAC-P3 $)=$ $5.010)$, i.e. a decline in agreement, in the case of data sets with multi-functional compounds. For the hightemperature range the average improvement is as follows: $35 \%$ (162 data sets; $F_{\text {obj, high- } T \text {, mono }}($ AIOMFAC-P3 $)=$ 31.237 ) for monofunctional and $43 \%$ (61 data sets; $F_{\text {obj, high- } T \text {,multi }}($ AIOMFAC-P3) $=6.317)$ for the data sets involving multi-functional compounds. Note that these percentages reflect a weighted average improvement of AIOMFAC-P3 (weighting depends on the $w_{d}^{\text {init }}$ values). There are some data sets for which the AIOMFAC-P3 parameterisation shows an improvement over AIOMFAC-P1 and in return there are some data sets for which the AIOMFAC-P1 parameterisation shows better agreement. In the case of the low-temperature range comparisons for the subset of data sets containing monofunctional compounds, AIOMFAC-P3 leads to improvement in the case of 34 data sets vs. a decline in the case of 12 data sets. For the low-temperature range subset of data sets containing multi-functional compounds, AIOMFAC-P3 leads to improvement in case of 50 data sets but to decline in case of 56 data sets.

Thus, while this evaluation shows that AIOMFAC-P3 leads to improvement with the experimental data considering the whole database, as well as for the subsets of low and high temperature ranges, the new parameterisation does also lead to a decline in agreement for a number of data sets with respect to the performance of the original AIOMFACP1 parameterisation. This is is partly due to the nature of applying a global parameter optimisation aiming at the simultaneous improvement of the weighted model-measurement deviations based on Eq. (22), which entails the possibility for reduced agreement for some systems as long as the overall model-measurement agreement increases. Moreover, the AIOMFAC-P1 parameterisation shows already good agreement with a part of the experimental data sets at low and high temperatures. For this fraction of data sets any changes in model prediction due to a new parameterisation may eas- 
a

$\mathrm{H}_{2} \mathrm{O}(1)+$ Malonic acid (2) Temperature range: $262-273 \mathrm{~K}$
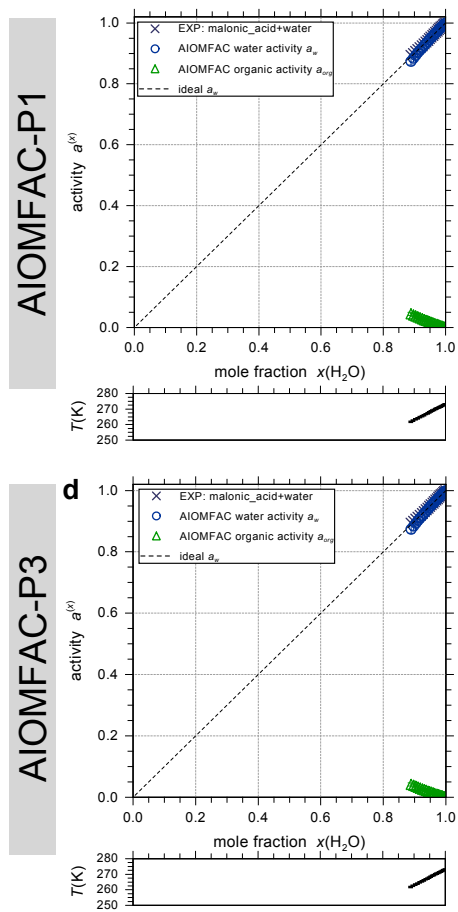

b

$\mathrm{H}_{2} \mathrm{O}(1)+$ Malonic acid (2) Temperature range: $278-338 \mathrm{~K}$
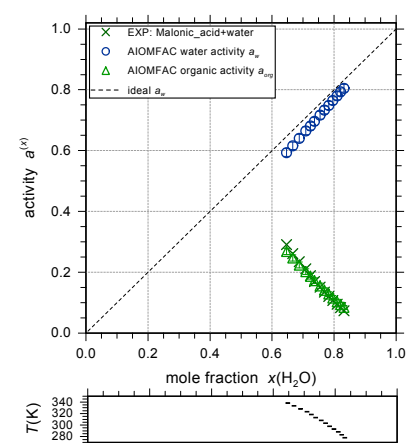

e

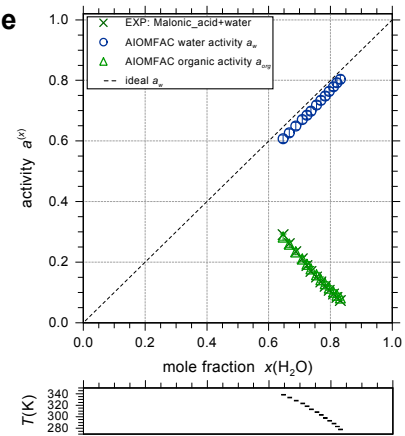

C

$\mathrm{H}_{2} \mathrm{O}(1)+$ Malonic acid (2)

Temperature range: $150-480 \mathrm{~K}$

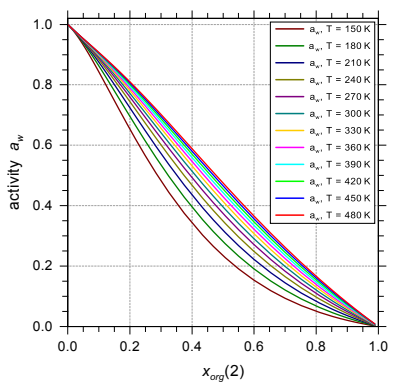

f

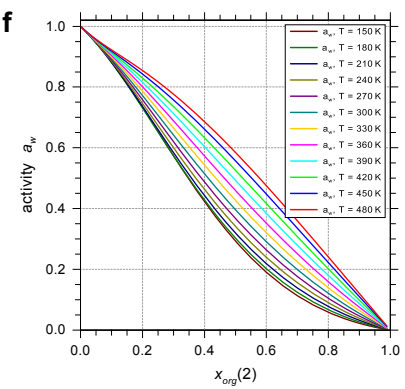

Figure 4. SLE measurements for water (1) + malonic acid (2) solutions and corresponding calculations of AIOMFAC-P1 (a-c) or AIOMFAC-P3 (d-f). Panels (c, f) show the temperature dependence of water activities predicted for the range 150-480 K. (a, d) Low temperature experimental SLE data (crosses) are compared with the predictions for water activity at the same compositions and temperatures (blue circles). Predictions of the corresponding organic activities are shown as well (green triangles) while (b, e) show analogous SLE data for the malonic acid melting curve. The error bars represent the model sensitivity to a composition variation by $x^{\text {tol }}=0.01$. The dashed line represents the hypothetical water activity of an ideal mixture. The temperatures of the individual data points are given in the boxes below the main panels. Experimental data: Braban et al. (2003) and Apelblat and Manzurola (1987).

ily lead to a decline rather than an improved agreement with experimental data. However, as long as the changes in the model predictions are small for these systems, a decline in agreement relative to AIOMFAC-P1 could still mean that AIOMFAC-P3 performs well. Nevertheless, there are also data sets for which both AIOMFAC parameterisations show relatively large discrepancies (e.g. the water + 2-butoxyethanol system further discussed below). For such systems, additional improvements of the AIOMFAC model are conceivable - either by a new fit of certain $a_{m, n}$ interaction parameters (kept untouched in this work) or by introduction of additional (special) subgroups that help to account for the effects of certain intra- and inter-molecular subgroupsubgroup interactions (e.g. intramolecular hydrogen-bonding among oxygenated functional groups in close proximity). A thorough evaluation of these options and improvement of AIOMFAC in this direction is the topic of future work.

In the following, the two AIOMFAC parameterisations are compared for a selection of aqueous organic mixtures and water-free organic mixtures. Again, it should be noted that the model was not just fitted to the selection of data sets shown; rather the figures show a few examples, and the AIOMFAC-P3 model is, of course, based on the simultaneous optimisation of all fit parameters to the complete database. For each individual system, a specific fit of either AIOMFAC-P1 or -P3 (to that data set only) would represent those data better, but is not the goal of a versatile groupcontribution model.

\subsection{Aqueous organic mixtures}

Figure 2 shows the comparison of aqueous 1,2-ethanediol solutions using the AIOMFAC-P1 and AIOMFAC-P3 models. Panels $(\mathrm{a}-\mathrm{c})$ represent the AIOMFAC-P1 performance while panels $(\mathrm{d}-\mathrm{f})$ represent the corresponding AIOMFAC-P3 results. The low-temperature SLE data (a and d) are well represented by both AIOMFAC-P1 and AIOMFAC-P3. The hightemperature VLE data are better represented by AIOMFACP3 in comparison to AIOMFAC-P1 even though both models show deviations from the experimental VLE-derived activity coefficients at low and high mole fraction of water. Panels (c) and (f) show predicted water activities covering the full concentration space from pure water to pure organic for 12 different temperature levels between $150 \mathrm{~K}$ and $480 \mathrm{~K}$. In 
a $\quad \mathrm{H}_{2} \mathrm{O}(1)+2$-Butanone (2) Temperature range: $198-273 \mathrm{~K}$
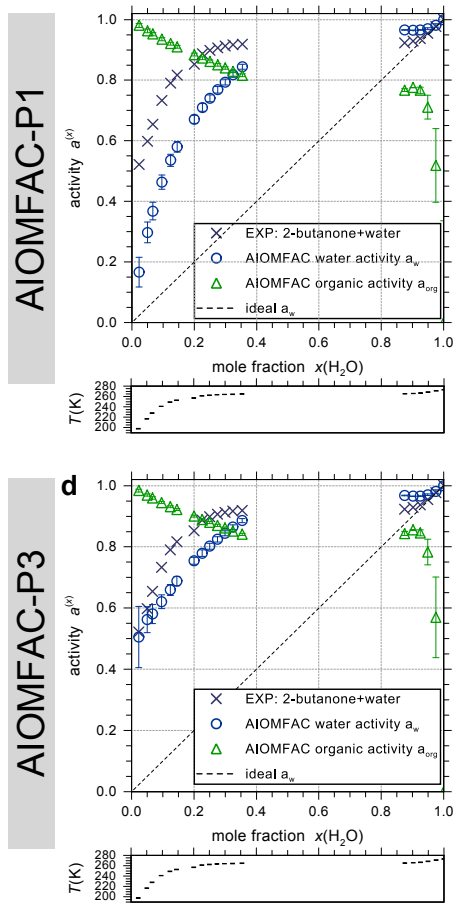

b

$\mathrm{H}_{2} \mathrm{O}(1)+2$-Butanone (2)
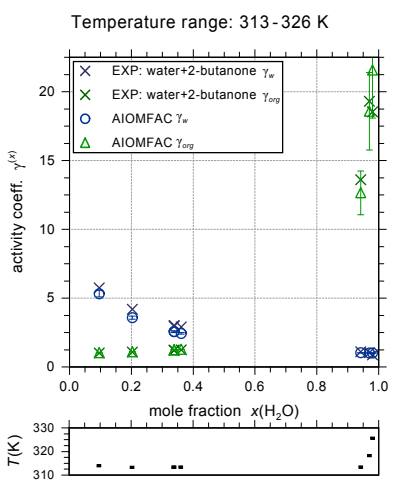

e

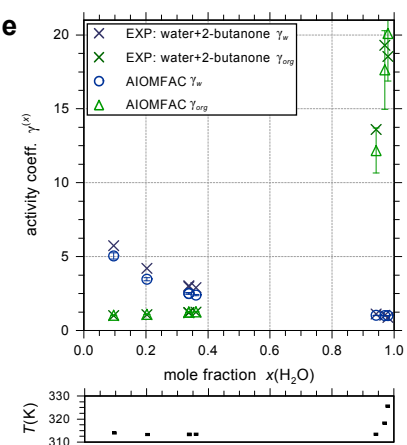

$\mathrm{H}_{2} \mathrm{O}(1)+2$-Butanone (2)

Temperature range: $150-480 \mathrm{~K}$

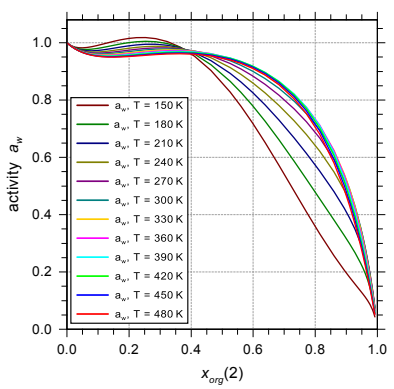

f

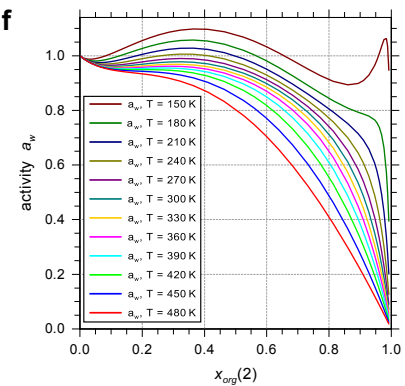

Figure 5. SLE and VLE measurements for water (1) + 2-butanone (2) solutions and corresponding calculations of AIOMFAC-P1 (a-c) or AIOMFAC-P3 (d-f). Panels (c, f) show the temperature dependence of water activities predicted for the range 150-480 K. (a, d) Low temperature experimental SLE data (crosses) are compared with the predictions for water activity at the same compositions and temperatures (blue circles). Predictions of the corresponding organic activities are shown as well (green triangles). The error bars represent the model sensitivity to a composition variation by $x^{\text {tol }}=0.01$. The dashed line represents the hypothetical water activity of an ideal mixture. Panels (b, e) show the model predictions of the activity coefficients compared to VLE data covering temperatures significantly higher than room temperature. The temperatures of the individual data points are given in the boxes below the main panels. Experimental data: Lohmann et al. (1997) and Gmehling et al. (1981).

comparison to AIOMFAC-P1, the resulting temperature dependence from low to high $x_{\text {org }}$ is slightly smaller in the case of the AIOMFAC-P3 parameterisation (f).

Figure 3 compares the model performance of AIOMFACP1 and AIOMFAC-P3 for SLE and VLE experimental data of aqueous acetic acid systems. The SLE data are well represented by both AIOMFAC-P1 and AIOMFAC-P3 (Fig. 3a and d). At higher temperatures, covered by VLE data, the AIOMFAC-P3 prediction is clearly in better agreement with the experimental data than the AIOMFAC-P1 calculation. The AIOMFAC-P1 parameterisation tends to underestimate the activity coefficients of water and acetic acid, particularly at high and low mole fractions of water. The extended description of the temperature dependence of activity coefficients in AIOMFAC-P3 allows a relatively good representation of observations at low and high temperatures, while AIOMFAC-P1 shows quite large deviations at higher temperatures. The temperature dependence predictions for the temperature range $150-480 \mathrm{~K}$ are given in panels (c, f). AIOMFAC-P1 predicts less pronounced temperature dependence at higher temperatures in the range 330-480 K.
This steeper slope of changes in water activity with temperature seems to be necessary to reproduce both VLE and SLE data for this system and other systems containing compounds with functional groups in common with the acetic acid + water system.

Figure 4 shows measured SLE data for the malonic acid + water system and its comparison with the predictions from AIOMFAC-P1 and AIOMFAC-P3. In the panels a and $\mathrm{c}$, water is the component in equilibrium with ice (hence the data describes the ice melting curve at different $T$ and mixture composition), while panels (b) and (e) show analogous data for the malonic acid melting curve. The temperature ranges are slightly different, with the highest temperature in the plots referring to the melting temperatures of the pure component or the SLE at the highest concentration of the organic component, respectively. The predicted $a_{\mathrm{w}}$ shows slight deviations from the experimental data towards lower water activities for both AIOMFAC-P1 and AIOMFAC-P3 $(\mathrm{a}, \mathrm{d})$. The predicted $a_{\text {org }}$ (in a range close to and above room temperature) is well represented in both AIOMFAC-P1 and AIOMFAC-P3 (b, e). No VLE data are available for aqueous 

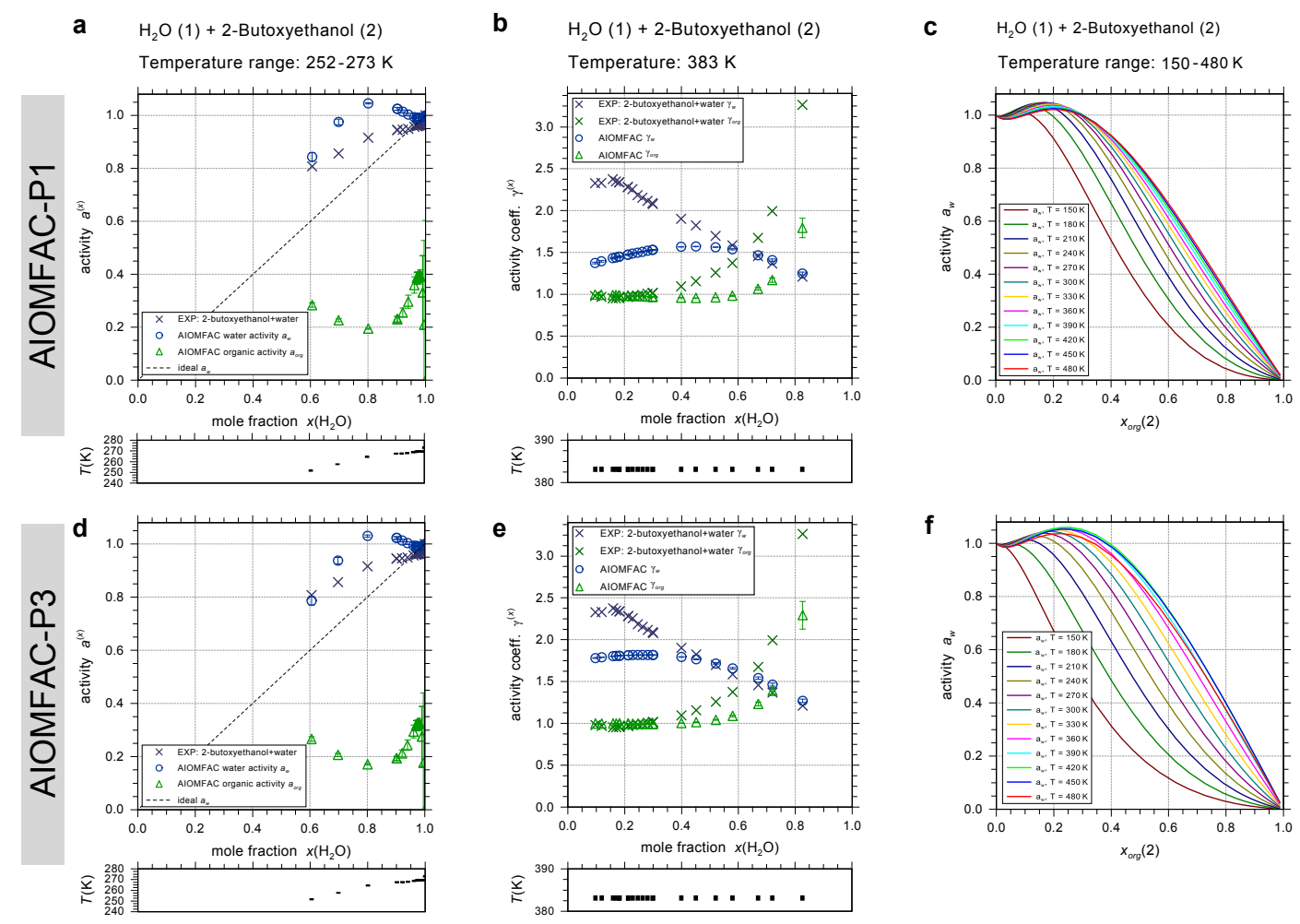

Figure 6. SLE and VLE measurements for 2-butoxyethanol + water solutions and corresponding calculations of AIOMFAC-P1 (a-c) or AIOMFAC-P3 (d-f). Panels (c, f) show the temperature dependence of water activities predicted for the range 150-480 K. (a, d) Low temperature experimental SLE data (crosses) are compared with the predictions for water activity at the same compositions and temperatures (blue circles). Predictions of the corresponding organic activities are shown as well (green triangles). The error bars represent the model sensitivity to a composition variation by $x^{\text {tol }}=0.01$. The dashed line represents the hypothetical water activity of an ideal mixture. Panels (b, e) show the model predictions of the activity coefficients compared to VLE data covering temperatures significantly higher than room temperature. The temperatures of the individual data points are given in the boxes below the main panels. Experimental data: Koga et al. (1994) and Schneider and Wilhelm (1959).

malonic acid at higher temperatures and hence could not be compared. AIOMFAC-P3 predicts a larger temperature dependence in comparison to AIOMFAC-P1, the latter shows a relatively small temperature dependence of water activity at higher temperatures (c, f).

Figure 5 shows an example of a binary system consisting of water and 2-butanone with a miscibility gap present over a large temperature and composition range. Both model parameterisations show deviations from the SLE data (a, d). However, the AIOMFAC-P3 parameterisation clearly reduces the deviations from the experimental data in comparison to AIOMFAC-P1, the latter showing deviations up to $>0.3$ in $a_{\mathrm{w}}$ at low water contents. On the other hand, at the higher temperatures covered by VLE data, both AIOMFAC-P1 (b) and AIOMFAC-P3 (e) are in good agreement with the experimental data. A miscibility gap is also predicted by both AIOMFAC parameterisations, although the width and temperature range of the predicted phase separations differ between the model results. According to the AIOMFAC-P3 prediction, a phase separation occurs in the temperature range 150 to $\sim 390 \mathrm{~K}$. The composition space where a liquid-liquid phase separation occurs is indicated in such diagrams by drawing a horizontal line (parallel to the abscissa) from one of the local minima of $a_{\mathrm{w}}$ to the point where it intersects with the $a_{\mathrm{w}}$ curve at a different $x_{\mathrm{org}}$ value. For example, for the green $a_{\mathrm{w}}$ curve at $T=180 \mathrm{~K}$, liquid-liquid phase separation is predicted at $a_{\mathrm{w}}=0.98$ in the composition range between $x_{\text {org }}$ (2-butanone) $=0.05$ and $x_{\text {org }}(2$-butanone $)=0.61$. A miscibility gap is also found in the experiments and is the reason why in panels $(\mathrm{a}, \mathrm{b}, \mathrm{d}, \mathrm{e})$ there are no data points in the mole fraction range $0.35<x$ $\left(\mathrm{H}_{2} \mathrm{O}\right)<0.85$.

Figure 6 shows the model-measurement comparison for aqueous 2-butoxyethanol. AIOMFAC-P1 and AIOMFAC-P3 show similar performance. Both models are not in good agreement with the experimental data. Contrary to the experimental data, both AIOMFAC-P1 and AIOMFAC-P3 pre$\operatorname{dict} a_{\mathrm{w}}>1$ in a certain composition range and both models predict a liquid-liquid phase separation over a wide range of temperatures, explaining the reason for deviations in predicted water activity shown in (a) and (d) (see also local minima in $a_{\mathrm{w}}$ curves of panels $\mathrm{c}, \mathrm{f}$ ). Note that the model 
a

Cyclohexanol (1) + Adipic acid (2)

Temperature range: $299-352 \mathrm{~K}$
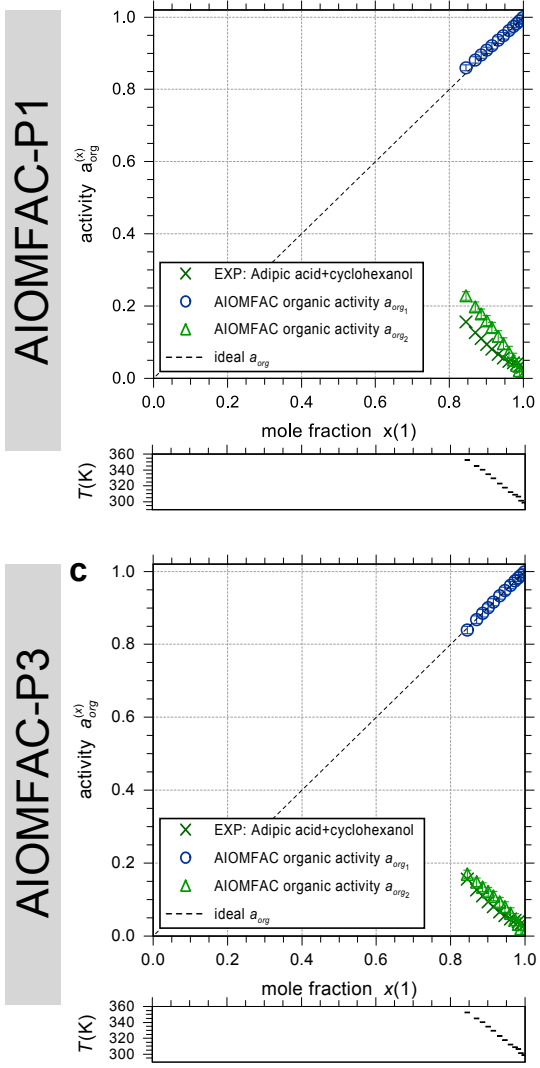

b

Cyclohexanol (1) + Adipicacid (2)

Temperature range: $150-480 \mathrm{~K}$

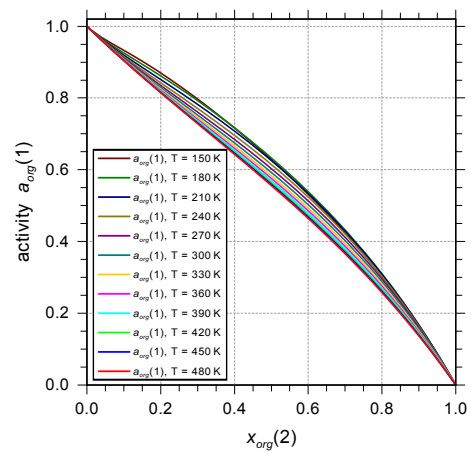

d

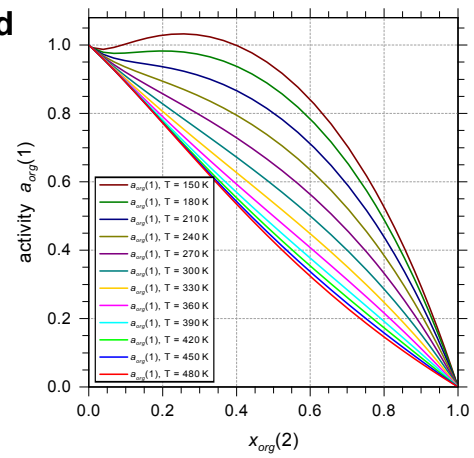

Figure 7. SLE measurements for cyclohexanol (1) + adipic acid (2) solutions and corresponding calculations of AIOMFAC-P1 (a, b) or AIOMFAC-P3 (c, d). Panels (b, d) represent the temperature dependence predictions from AIOMFAC-P1 and AIOMFAC-P3 for the temperature range 150-480 K. (a, c) SLE of adipic acid shown vs. mole fraction of cyclohexanol (component 1). The error bars represent the model sensitivity to a composition variation by $x^{\text {tol }}=0.01$. The dashed line is the ideal solution curve for component 1 . The temperatures of the individual data points are given in the boxes below the main panels. Experimental data: Lihua et al. (2007).

predictions in these figures do not include phase separation computations on purpose, since the experimental data are for a homogeneous single phase (no phase separation found experimentally) so are the model calculations here. Also, at higher temperatures the activity coefficients of both water and 2-butoxyethanol show deviation from experimental data (b, e). AIOMFAC-P3 shows a larger temperature dependence over the entire temperature range in comparison to AIOMFAC-P1 (c, f). The observed disagreement between both models and the experimental data is mainly due to the fact that there is already a clear discrepancy between AIOMFAC (both versions) and the experimental data near room temperature. Since there is already disagreement at the reference temperature $(298.15 \mathrm{~K})$, the new model parameters for improved temperature dependence $\left(b_{m, n}\right.$ and $\left.c_{m, n}\right)$ cannot (and should not) remove this model-measurement discrepancy. This system illustrates that a re-parameterisation of certain $a_{m, n}$ group interaction parameters may be necessary to improve AIOMFAC for this and similar systems.

\subsection{Binary organic mixtures}

Figure 7 shows the model-measurement comparison for SLE data of the water-free mixture of cyclohexanol + adipic acid. The AIOMFAC-P3 prediction is in better agreement with the experimental data than AIOMFAC-P1, which shows a positive deviation at lower mole fractions of component 1 (cyclohexanol). In this binary system, the AIOMFAC-P3 parameterisation leads to a relatively large temperature dependence of the activity of cyclohexanol, $a_{\mathrm{org}}(1)$, (panel d). In addition, with that parameterisation a phase separation occurs at lower $x_{\text {org }}(2)$ values for temperatures below $\sim 180 \mathrm{~K}$. However, no phase separation is expected at higher temperatures, more relevant in the troposphere. AIOMFAC-P1 on the other hand shows a much smaller temperature dependence (b) and does not predict a phase separation in the range shown.

Measurements for water-free binary organic mixtures of ethanol+acetone are shown in Fig. 8. The AIOMFAC-P3 predictions of the activities of acetone are in a very good 
Ethanol (1) + Acetone (2) Temperature range: $164-173 \mathrm{~K}$
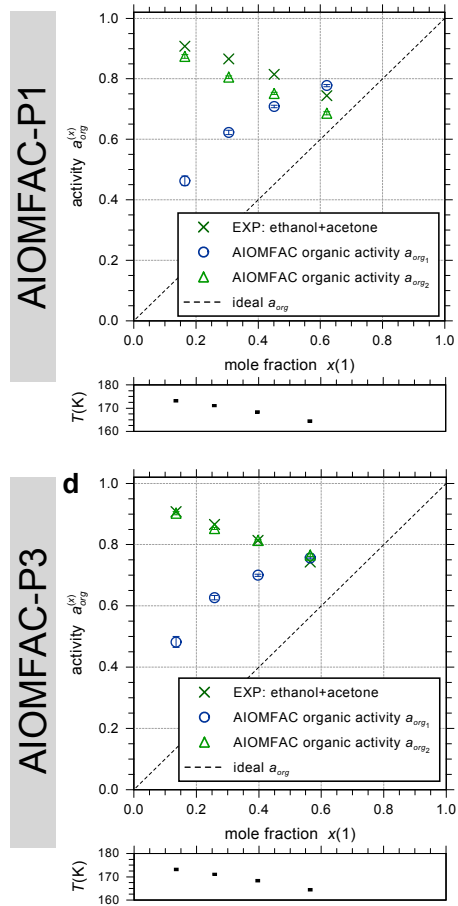

b

Ethanol (1) + Acetone (2) Temperature range: $330-350 \mathrm{~K}$
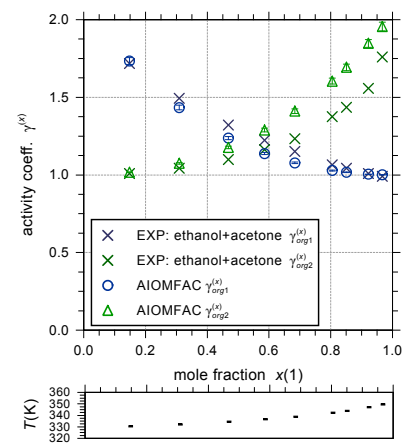

e

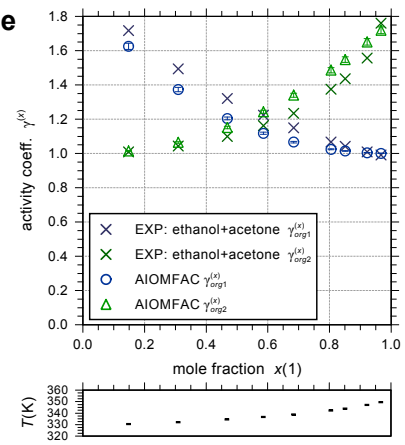

Ethanol (1) + Acetone (2)

Temperature range: $150-480 \mathrm{~K}$

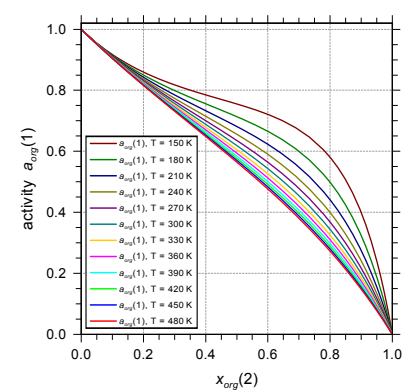

f

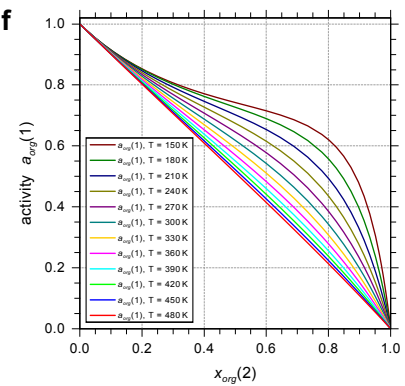

Figure 8. SLE and VLE measurements for ethanol (1) + acetone (2) solutions and corresponding calculations of AIOMFAC-P1 (a-c) or AIOMFAC-P3 (d-f). Panels (c, f) show the temperature dependence as predicted by AIOMFAC-P1 and AIOMFAC-P3 for the temperature range $150-480 \mathrm{~K}$. (a, d) Low temperature experimental SLE data (crosses), shown as mole fraction of ethanol, x(1), vs. activity $\left(a_{\text {org2 }}^{(x)}\right)$ of acetone. The error bars represent the model sensitivity to a composition variation by $x^{\text {tol }}=0.01$. The dashed line is the ideal solution curve for component 1 . Panels $(\mathbf{b}, \mathbf{e})$ show the model predictions of the activity coefficients compared to VLE data covering temperatures significantly higher than room temperature. The temperatures of the individual data points are given in the boxes below the main panels. Experimental data: Sapgir (1929) and Amer et al. (1956).

agreement with the experimental SLE-derived data (d), while AIOMFAC-P1 shows larger deviations from the experimental data at these low temperatures (a). At high temperatures, the VLE data for both AIOMFAC-P1 and AIOMFAC-P3 show similar results $(b, e)$, with slightly larger deviations of $\gamma_{\mathrm{org}_{2}}^{(x)}$ (activity coefficient of component 2, i.e. acetone) in the predictions of AIOMFAC-P1. At temperatures higher than $300 \mathrm{~K}$ both AIOMFAC-P1 and AIOMFAC-P3 show a much smaller temperature dependence than for the range below room temperature.

Figure 9 shows a similar example for ethanol+3heptanone mixtures. The prediction from AIOMFAC-P3 is in relatively good agreement with the experimental SLE data, showing less deviations in 3-heptanone activities than the results from the AIOMFAC-P1 calculations. Achieving better agreement with the new (AIOMFAC-P3) parameterisation requires a larger temperature dependence of the organic activities, particularly towards lower temperatures (d).

Figure 10 shows the binary ethanol + diethyl ether system, where experimental data are available for a temperature range spanning more than $200 \mathrm{~K}$ : from $149 \mathrm{~K}$ up to $378 \mathrm{~K}$. Of course, additional data from other systems of our database are also affecting the main group interaction parameters that are necessary to describe this system with AIOMFAC-P3. Both models describe the diethyl ether activity derived from SLE at low temperatures quite well (a and d). AIOMFACP3 shows slight overprediction of the diethyl ether activity in the range $0.15<x$ (ethanol) $<0.6$, while AIOMFAC-P1 tends to underpredict the experimental data. In contrast, at higher temperatures ( $~ 350$ to $380 \mathrm{~K})$ covered by experimental VLE data (b and e), the predicted activity coefficients $\gamma_{\text {org }}^{(x)}$ (diethyl ether) at high mole fractions of ethanol (component 1) both by AIOMFAC-P1 and AIOMFAC-P3 are not in good agreement with the VLE experimental data. The main reason for the observed deviations is due to inaccurately predicted activity coefficients at infinite dilution (i.e. when one of the compounds is present only as a tiny mole fraction in the solution) of the two organic compounds at these temperatures. At infinite dilution conditions the activity coefficients are dominated by subgroup volume and surface area properties in the UNIFAC/AIOMFAC model, so that the activity coefficient values are largely unaffected by the new main 
a

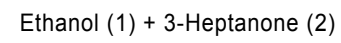

Temperature range: $204-236 \mathrm{~K}$
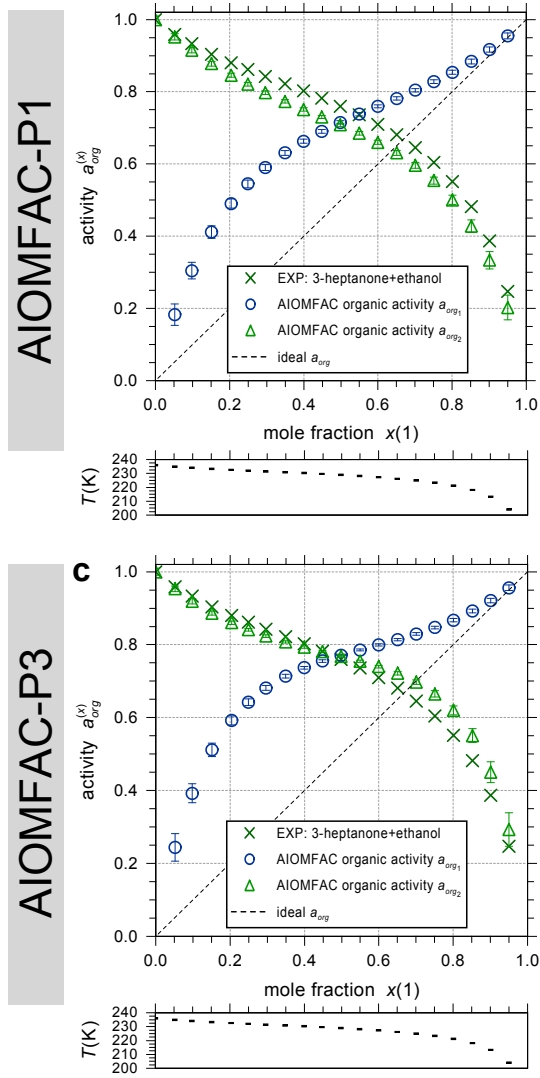

b

Ethanol (1) + 3-Heptanone (2)

Temperature range: $150-480 \mathrm{~K}$

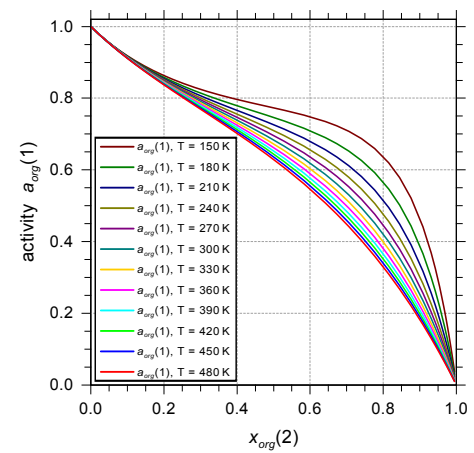

d

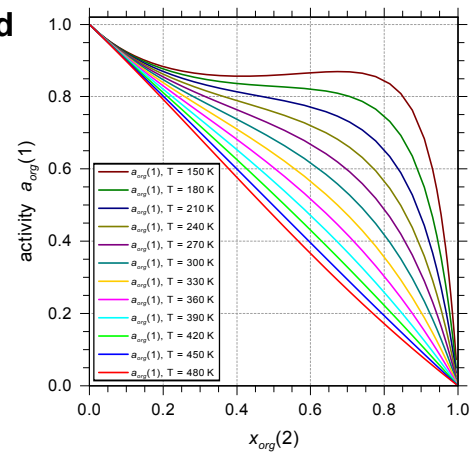

Figure 9. SLE measurements for ethanol (1) + 3-heptanone (2) solutions and corresponding calculations of AIOMFAC-P1 (a, b) or AIOMFAC-P3 (c, d). Panels (b, d) show the temperature dependence predictions from AIOMFAC-P1 and AIOMFAC-P3 for the temperature range 150-480 K. The SLE data in (a, c) show the composition (mole fraction of ethanol) against activity of 3-heptanone. The error bars represent the model sensitivity to a composition variation by $x^{\text {tol }}=0.01$. The dashed line is the ideal solution curve for component 1 . Experimental data: Fiege et al. (1996).

group interaction parameterisation of AIOMFAC-P3 in comparison to AIOMFAC-P1. As is visible from panels (c) and (f), particularly at $x$ (diethyl ether) $>0.4$, the temperature dependence of ethanol activities predicted by AIOMFAC-P3 is larger than the original one in AIOMFAC-P1. The example of this system shows that it is not always possible to achieve good model predictions for the full temperature range with the new treatment of temperature dependence in AIOMFAC. For further improvements, other model parts, such as the lattice constant $(z)$, which is not really a constant, may need to be considered for the introduction of additional, physically meaningful temperature dependent parameterisations.

\subsection{Scope and limitations of the new parameterisation}

The thermodynamic model AIOMFAC has been developed based on modified versions of UNIFAC and LIFAC, with the aim to establish a versatile activity coefficient model for atmospheric applications. The new parameterisation of the model aims at improving AIOMFAC predictions particularly at lower temperatures of atmospheric relevance. Deviations between the experimental data and model predictions from the new AIOMFAC-P3 version are associated with either the inaccuracy of the measurements, the lack of data to better cover and parameterise the model for a wide composition and temperature range, or limitations of the AIOMFAC expressions and the group contribution method. Own measurements were performed for selected aqueous organic systems at low temperatures and at temperatures around room temperature, which were used together with experimental data from the literature for parameterising the model over a wider temperature range.

While an extensive database was compiled to allow for an improvement of the AIOMFAC model with respect to its performance at temperatures substantially lower and higher than room temperature, there are still limitations present in the general coverage of the extended temperature range by experimental data. The complexity of organic molecules 


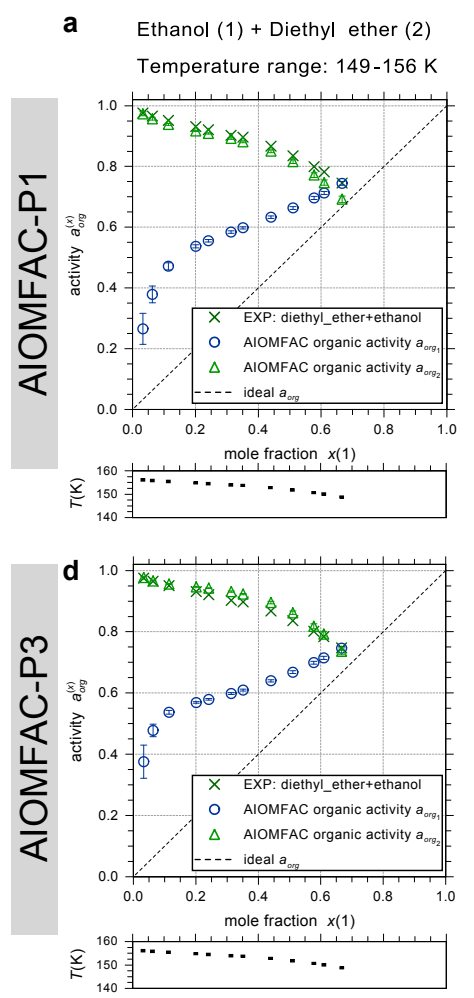

b

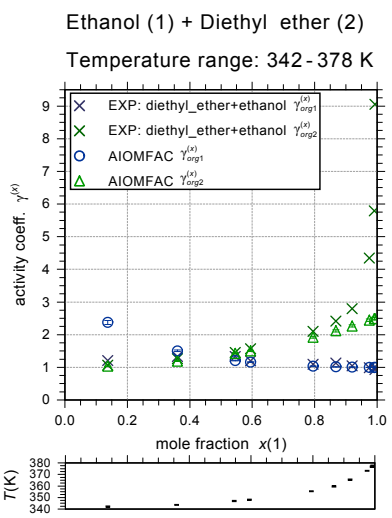

e

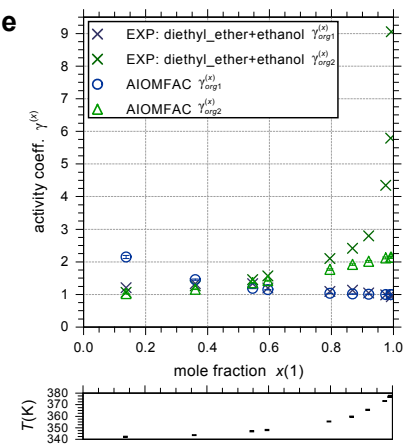

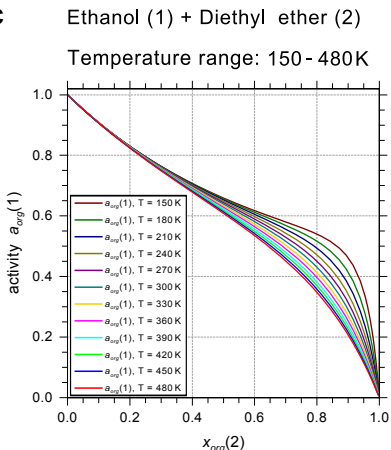

f

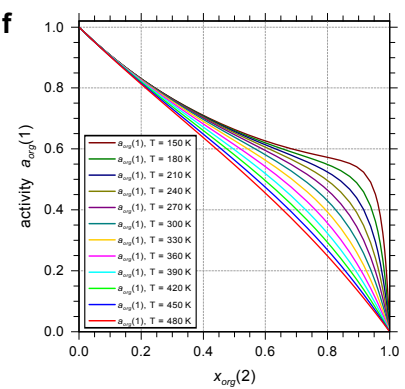

Figure 10. SLE and VLE measurements for ethanol (1) + diethyl ether (2) solutions and corresponding calculations of AIOMFAC-P1 (a-c) or AIOMFAC-P3 (d-f). Panels (c, f) show the temperature dependence of the ethanol activity, as predicted by AIOMFAC-P1 and AIOMFAC-P3 for the temperature range 150-480 K. (a, d) Experimental SLE data (crosses) compared with model predictions (triangles) for the activity of diethyl ether in the very low temperature range 149 to $156 \mathrm{~K}$. The dashed line is the ideal solution curve for component 1 . Panels (b, e) show the model predictions of the activity coefficients compared to VLE data covering temperatures significantly higher than room temperature. The temperatures of the individual data points are given in the boxes below the main panels. The error bars represent the model sensitivity to a composition variation by $x^{\text {tol }}=0.01$. Experimental data: Lalande (1934) and Moeller et al. (1951).

in terms of their physical and chemical properties such as size, shape and combinations of functional groups, particularly the number and proximity of oxygen-bearing functionalities, are important factors that influence the quality of AIOMFAC predictions. Of interest for atmospheric aerosol systems are data sets containing multi-functional organic compounds, such as sugar-like compounds, di- and polycarboxylic acids, hydroxylated ketones and functionalized aromatic compounds. We have added many experimental thermodynamic equilibrium data sets that cover systems containing such compounds (e.g. sorbitol, 1,2,7,8octanetetrol, sucrose, raffinose, citric acid, malonic acid, 2isopropoxyethanol, vanillylmandelic acid, etc.; see Table 1 for a complete list), yet overall the database remains dominated by small, monofunctional organic compounds. This possibly limits the accuracy of AIOMFAC-P3 for predicting activity coefficients in multi-component systems containing multi-functional, high-molecular mass species. This is a disadvantage not just for AIOMFAC-P3 or this work, but generally rooted in the very limited amount of experimental data covering such systems (especially at temperatures much lower/higher than $298 \mathrm{~K}$ ). Due to this, the accuracy of
AIOMFAC predictions is expected to decrease with increasing complexity of organic compounds.

As discussed in more detail at the beginning of Sect. 5, it is found that the new temperature dependence parameterisation shows particular improvement for the majority of systems containing monofunctional compounds both in the low and high temperature ranges (the temperature ranges at least $25 \mathrm{~K}$ above/below $298 \mathrm{~K}$ ). In case of the mixtures containing multi-functional compounds present in our database, the improvement for the low-temperature range in comparison to AIOMFAC-P1 is more diverse: for about half of the data sets a small improvement is found while for the other half a reduced agreement is the result of the parameter optimisation. For some systems containing multi-functional compounds, the model-measurement agreement is not good with either AIOMFAC version (e.g. in case of the aqueous 2butoxyethanol system shown in Fig. 6). For such systems and corresponding main group interactions, further improvement of AIOMFAC may only be achieved by a refitting of certain $a_{m, n}$ interaction parameters involved.

It is known that the standard UNIFAC parameterisation and model expressions need to be modified for aque- 
ous oligomer/polymer solutions to achieve good modelmeasurement agreement (e.g. Ninni et al., 1999). This has been done in previous work targeting specific types of polymers, including the introduction of specific UNIFAC groups fitted to experimental data exclusively of such polymer systems (e.g. Ninni et al., 1999). The introduced AIOMFACP3 parameterisation has as purpose general applicability, as is the case for AIOMFAC-P1. Therefore, it is expected that the AIOMFAC model may perform rather poorly when used for predicting activity coefficients in oligomer/polymer solutions. For such systems, the application of specifically fitted models is recommended. However, the new AIOMFAC parameterisation provides a tool to predict activity coefficients with better overall accuracy than the previous version and offers the versatility of a group-contribution method for the prediction of activity coefficients in complex mixtures containing many tens to thousands of individual components.

\section{Conclusions}

An improved temperature dependence parameterisation of aqueous organic and water-free organic mixtures is presented for the thermodynamic group contribution model AIOMFAC. A comprehensive database of experimental thermodynamic equilibria data is established by collecting and carefully validating different data types covering a wide temperature and concentration range. In addition, new measurements that have been performed for selected aqueous organic systems, at room temperature and below, were also included in the database. The database is used to determine new AIOMFAC group interaction parameters for organic main groups of atmospheric relevance: carboxyl, hydroxyl, ketone, aldehyde, ether, ester, alkyl, aromatic carbonalcohol, and aromatic hydrocarbons. The parameter fitting procedure involved the simultaneous determination of 150 interaction parameters for the 14 main groups. Thus, the new temperature dependence parameterisation allows to calculate activity coefficients and their temperature dependence for a wide variety of organic and water-free mixtures. In general, the new AIOMFAC parameterisation achieves good agreement with a large number of experimental data sets. In the case of some organic systems, lack of experimental data to constrain the activity coefficients is a major limitation. Further improvements of the AIOMFAC model description of these systems and by that, the interactions of the functional groups involved, will require additional measurements over a wide temperature and concentration range. In addition, larger discrepancies in model-measurement agreement were found in particular for some of the systems containing multi-functional organic compounds. The affected systems were typically also poorly represented at room temperature. Further investigations will be needed to thoroughly address these issues and achieve better performance of AIOMFAC in such cases over the full temperature range of interest.
The performance of the AIOMFAC parameterisation is typically better for systems containing relatively small organic compounds and substantial deviations may occur in mixtures when molecules of high structural complexity such as highly oxygenated compounds or molecules of high molecular mass (e.g. oligomers and polymers) prevail. The improved AIOMFAC model can be used to better account for the temperature dependence of activity coefficients relevant in predictions related to atmospheric ice nucleation and gas-particle partitioning in multi-component systems. 


\section{Appendix A}

Bulk water activities, $a_{\mathrm{w}}$, were measured for aqueous organic solutions using an AquaLab water activity meter (Model 3TE, Decagon devices, USA). The instrument applies the chilled mirror technology to determine the dewpoint temperature of air equilibrated with the aqueous solution being measured which is then translated to water activity. The instrument's infrared thermometry indicates the sample temperature, which is then considered in the determination of water activity. So, accurate measurements are not dependent on precise thermal equilibrium at a set temperature level. The internal temperature control allows to perform measurements under stable temperature from 289$313 \mathrm{~K}$. The standard sample block with a specified error of \pm 0.003 in $a_{\mathrm{w}}$ (absolute range) was used for most experiments. For the more volatile polyols (2,5-hexanediol, 1,2,6hexanetriol, and glycerol) the volatile sample block available using a hygroscopic polymer sensor to detect the equilibrium relative humidity of air in the headspace above the sample was used to perform measurements. The stated error for the volatile sample block is \pm 0.015 in $a_{\mathrm{w}}$ (absolute range). Instrument offset is frequently corrected and the performance of the sample block was controlled and readjusted with reference samples. All measurements were performed at $289-313 \mathrm{~K}$. The substances were purchased from Sigma-Aldrich in the best available purity. The following solutes were investigated: glycerol (Sigma, > 99\%), 2,5-hexanediol (Fluka, > $97 \%$ ), 1,2,6-hexanetriol (Fluka, $>95 \%$ ), 1,2,7,8-octanetetrol (Fluka, > $97 \%$ ), 2,2,6,6tetrakis(hydroxymethyl)cyclohexanol (Aldrich, 97\%), DL4-hydroxy-3-methoxy mandelic acid ("vanillylmandelic acid"; Sigma, > $95 \%$ ), raffinose (Sigma, > $98 \%$ ). The substances were used without further purification. The water/polyol mixtures were prepared by mass percent with MilliQ water using an analytical balance. Each solution was measured at least three times at each temperature. Water activity data for these aqueous solutions are tabulated in Appendix Tables A1-A8. 
Table A1. Bulk water activity $\left(a_{\mathrm{w}}\right)$ measurements ${ }^{\mathrm{a}}$ of water $(1)+$ glycerol (2) solutions at three different temperatures. Solution compositions are given in mole fraction of the organic component (2), $x_{2}$.

\begin{tabular}{cccc}
\hline$x_{2}$ & $\begin{array}{c}a_{\mathrm{W}} \\
(T=289.15 \mathrm{~K})\end{array}$ & $\begin{array}{c}a_{\mathrm{W}} \\
(T=298.15 \mathrm{~K})\end{array}$ & $\begin{array}{c}a_{\mathrm{W}} \\
(T=313.15 \mathrm{~K})\end{array}$ \\
\hline 0.01769 & 0.976 & 0.980 & 0.980 \\
0.03477 & 0.964 & 0.964 & 0.970 \\
0.05128 & 0.956 & 0.953 & 0.955 \\
0.06721 & 0.937 & 0.935 & 0.940 \\
0.08263 & 0.916 & 0.920 & 0.920 \\
0.09754 & 0.896 & 0.895 & 0.910 \\
0.11199 & 0.872 & 0.875 & 0.883 \\
0.12595 & 0.854 & 0.862 & 0.864 \\
0.13950 & 0.838 & 0.841 & 0.856 \\
0.15263 & 0.823 & 0.826 & 0.833 \\
0.16960 & 0.802 & 0.802 & 0.815 \\
0.22685 & 0.728 & 0.732 & 0.739 \\
0.31338 & 0.622 & 0.628 & 0.628 \\
0.43896 & 0.492 & 0.491 & 0.497 \\
0.63774 & 0.297 & 0.298 & 0.299 \\
\hline
\end{tabular}

${ }^{\mathrm{a}}$ The accuracy of the water activity measurements is specified as \pm 0.015 (absolute range) in $a_{\mathrm{W}}$.

Table A2. Bulk water activity $\left(a_{\mathrm{W}}\right)$ measurements ${ }^{\mathrm{a}}$ of water $(1)+2,5$-hexanediol (2) solutions at three different temperatures. Solution compositions are given in mole fraction of the organic component (2), $x_{2}$.

\begin{tabular}{cccc}
\hline$x_{2}$ & $\begin{array}{c}a_{\mathrm{W}} \\
(T=289.15 \mathrm{~K})\end{array}$ & $\begin{array}{c}a_{\mathrm{W}} \\
(T=298.15 \mathrm{~K})\end{array}$ & $\begin{array}{c}a_{\mathrm{W}} \\
(T=313.15 \mathrm{~K})\end{array}$ \\
\hline 0.0167 & 0.971 & 0.978 & 0.975 \\
0.0365 & 0.974 & 0.978 & 0.973 \\
0.0616 & 0.943 & 0.955 & 0.972 \\
0.0934 & 0.917 & 0.937 & 0.953 \\
0.1325 & 0.897 & 0.912 & 0.933 \\
0.1790 & 0.882 & 0.894 & 0.912 \\
0.2734 & 0.825 & 0.849 & 0.860 \\
0.3607 & 0.781 & 0.790 & 0.804 \\
0.5630 & 0.605 & 0.620 & 0.618 \\
\hline
\end{tabular}

a The accuracy of the water activity measurements is specified as \pm 0.015 (absolute range) in $a_{\mathrm{w}}$.

Table A3. Bulk water activity $\left(a_{\mathrm{w}}\right)$ measurements ${ }^{\mathrm{a}}$ of water $(1)+1,2,6$-hexanetriol (2) solutions at three different temperatures. Solution compositions are given in mole fraction of the organic component (2), $x_{2}$.

\begin{tabular}{cccc}
\hline$x_{2}$ & $\begin{array}{c}a_{\mathrm{W}} \\
(T=289.15 \mathrm{~K})\end{array}$ & $\begin{array}{c}a_{\mathrm{W}} \\
(T=298.15 \mathrm{~K})\end{array}$ & $\begin{array}{c}a_{\mathrm{W}} \\
(T=313.15 \mathrm{~K})\end{array}$ \\
\hline 0.014 & 0.975 & 0.988 & 0.985 \\
0.032 & 0.957 & 0.974 & 0.973 \\
0.055 & 0.944 & 0.962 & 0.966 \\
0.080 & 0.919 & 0.934 & 0.943 \\
0.114 & 0.890 & 0.895 & 0.909 \\
0.171 & 0.834 & 0.847 & 0.853 \\
0.216 & 0.784 & 0.802 & 0.802 \\
0.340 & 0.664 & 0.673 & 0.681 \\
0.539 & 0.456 & 0.458 & 0.465 \\
\hline
\end{tabular}

a The accuracy of the water activity measurements is specified as \pm 0.015 (absolute range) in $a_{\mathrm{w}}$. 
Table A4. Bulk water activity $\left(a_{\mathrm{w}}\right)$ measurements ${ }^{\mathrm{a}}$ of water $(1)+1,2,7,8$-octanetetrol $(2)$ solutions at three different temperatures. Solution compositions are given in mole fraction of the organic component (2), $x_{2}$.

\begin{tabular}{cccc}
\hline$x_{2}$ & $\begin{array}{c}a_{\mathrm{W}} \\
(T=289.15 \mathrm{~K})\end{array}$ & $\begin{array}{c}a_{\mathrm{W}} \\
(T=298.15 \mathrm{~K})\end{array}$ & $\begin{array}{c}a_{\mathrm{W}} \\
(T=313.15 \mathrm{~K})\end{array}$ \\
\hline 0.0109 & 0.987 & 0.988 & 0.993 \\
0.0245 & 0.976 & 0.977 & 0.981 \\
0.0407 & 0.963 & 0.965 & 0.969 \\
0.0650 & 0.944 & 0.946 & 0.953 \\
0.0878 & 0.927 & 0.924 & 0.933 \\
0.1329 & 0.877 & 0.887 & 0.901 \\
0.1890 & 0.803 & 0.817 & 0.837 \\
0.2911 & 0.605 & 0.643 & 0.667 \\
\hline
\end{tabular}

a The accuracy of the water activity measurements is specified as \pm 0.015 (absolute range) in $a_{\mathrm{w}}$.

Table A5. Bulk water activity $\left(a_{\mathrm{w}}\right)$ measurements ${ }^{\mathrm{a}}$ of water $(1)+2,2,6,6$-tetrakis(hydroxymethyl)-cyclohexanol (2) solutions at three different temperatures. Solution compositions are given in mole fraction of the organic component (2), $x_{2}$.

\begin{tabular}{cccc}
\hline$x_{2}$ & $\begin{array}{c}a_{\mathrm{W}} \\
(T=289.15 \mathrm{~K})\end{array}$ & $\begin{array}{c}a_{\mathrm{W}} \\
(T=298.15 \mathrm{~K})\end{array}$ & $\begin{array}{c}a_{\mathrm{W}} \\
(T=313.15 \mathrm{~K})\end{array}$ \\
\hline 0.0999 & 0.990 & 0.992 & 0.993 \\
0.1943 & 0.987 & 0.982 & 0.990 \\
0.3029 & 0.973 & 0.974 & 0.979 \\
0.3963 & 0.961 & 0.964 & 0.968 \\
0.5010 & 0.929 & 0.938 & 0.942 \\
0.6000 & 0.900 & 0.909 & 0.916 \\
0.6519 & 0.881 & 0.887 & 0.895 \\
0.7065 & 0.821 & 0.828 & 0.840 \\
\hline
\end{tabular}

a The accuracy of the water activity measurements is specified as \pm 0.015 (absolute range) in $a_{\mathrm{w}}$.

Table A6. Bulk water activity $\left(a_{\mathrm{w}}\right)$ measurements ${ }^{\mathrm{a}}$ of water $(1)+$ vanillylmandelic acid (2) solutions at three different temperatures. Solution compositions are given in mole fraction of the organic component (2), $x_{2}$.

\begin{tabular}{cccc}
\hline$x_{2}$ & $\begin{array}{c}a_{\mathrm{W}} \\
(T=289.15 \mathrm{~K})\end{array}$ & $\begin{array}{c}a_{\mathrm{W}} \\
(T=298.15 \mathrm{~K})\end{array}$ & $\begin{array}{c}a_{\mathrm{W}} \\
(T=313.15 \mathrm{~K})\end{array}$ \\
\hline 0.0102 & 0.997 & 0.999 & 0.996 \\
0.0354 & 0.981 & 0.987 & 0.985 \\
0.0844 & 0.963 & 0.965 & 0.965 \\
0.1201 & 0.940 & 0.945 & 0.949 \\
0.1712 & 0.891 & 0.898 & 0.906 \\
0.2107 & 0.851 & 0.857 & 0.860 \\
\hline
\end{tabular}

a The accuracy of the water activity measurements is specified as \pm 0.015 (absolute range) in $a_{\mathrm{w}}$. 
Table A7. Bulk water activity $\left(a_{\mathrm{w}}\right)$ measurements ${ }^{\mathrm{a}}$ of water $(1)+$ raffinose (2) solutions at three different temperatures. Solution compositions are given in mole fraction of the organic component (2), $x_{2}$.

\begin{tabular}{cccc}
\hline$x_{2}$ & $\begin{array}{c}a_{\mathrm{W}} \\
(T=289.15 \mathrm{~K})\end{array}$ & $\begin{array}{c}a_{\mathrm{W}} \\
(T=298.15 \mathrm{~K})\end{array}$ & $\begin{array}{c}a_{\mathrm{W}} \\
(T=313.15 \mathrm{~K})\end{array}$ \\
\hline 0.0089 & 0.993 & 0.993 & 0.992 \\
0.0232 & 0.967 & 0.969 & 0.973 \\
0.0364 & 0.938 & 0.944 & 0.948 \\
0.0507 & 0.910 & 0.913 & 0.917 \\
0.0781 & & 0.835 & \\
\hline
\end{tabular}

a The accuracy of the water activity measurements is specified as \pm 0.003 (absolute range) in $a_{\mathrm{W}}$.

Table A8. Bulk water activity $\left(a_{\mathrm{W}}\right)$ measurements ${ }^{\mathrm{a}}$ of water $(1)+$ sucrose $^{2}$ ) solutions at three different temperatures. Solution compositions are given in mole fraction of the organic component (2), $x_{2}$.

\begin{tabular}{cccc}
\hline$x_{2}$ & $\begin{array}{c}a_{\mathrm{w}} \\
(T=289.15 \mathrm{~K})\end{array}$ & $\begin{array}{c}a_{\mathrm{W}} \\
(T=298.15 \mathrm{~K})\end{array}$ & $\begin{array}{c}a_{\mathrm{W}} \\
(T=313.15 \mathrm{~K})\end{array}$ \\
\hline 0.0104 & 0.992 & 0.992 & 0.998 \\
0.0162 & 0.981 & 0.988 & 0.992 \\
0.0230 & 0.977 & 0.977 & 0.985 \\
0.0306 & 0.965 & 0.971 & 0.977 \\
0.0394 & 0.952 & 0.955 & 0.963 \\
0.0487 & 0.938 & 0.939 & 0.946 \\
0.0606 & 0.906 & 0.914 & 0.922 \\
0.0732 & 0.883 & 0.888 & 0.893 \\
\hline
\end{tabular}

a The accuracy of the water activity measurements is specified as \pm 0.003 (absolute range) in $a_{\mathrm{w}}$. 
Acknowledgements. This work was supported by the Swiss National Foundation, project 200020-125151 and by the CCES projects IMBALANCE and OPTIWARES funded by the ETH Domain.

Edited by: B. Ervens

\section{References}

Abbas, R. and Gmehling, J.: Vapour-liquid equilibria, azeotropic data, excess enthalpies, activity coefficients at infinite dilution and solid-liquid equilibria for binary alcohol-ketone systems, Fluid Phase Equilibr., 267, 119-126, 2008.

Ablett, S., Izzard, M., and Lillford, P.: Differential scanning calorimetric study of frozen sucrose and glycerol solutions, J. Chem. Soc. Faraday T., 88, 789-794, doi:10.1039/FT9928800789, 1992.

Abrams, D. S. and Prausnitz, J. M.: Statistical thermodynamics of liquid mixtures: a new expression for the excess Gibbs energy of partly or completely miscible systems, AIChE J., 21, 116-128, 1975.

Ahlers, J.: Measurement, modeling and evaluation of solidliquid phase equilibria, Frankfurt/Main: DECHEMA Deutsche Gesellschaft für Chemisches Apparatewesen, 126 pp., 1998.

Al-Muhtaseb, S. and Fahim, M.: Phase equilibria of the ternary system water/acetic acid/2-pentanol, Fluid Phase Equilibr., 123, 189-203, 1996.

Al-Rub, F., Abdel-Jabbar, N., Darwish, N., and Ghanem, H.: Vaporliquid equilibrium data for the 2-methoxy-2-methylpropane (MTBE)-ethanol, MTBE-ethanol-calcium chloride, and MTBE-ethanol-copper chloride, Separ. Sci. Technol., 37, 1911-1926, doi:10.1081/SS-120003051, 2002.

Alexander, D.: The solubility of benzene in water, J. Phys. Chem., 63, 1021-1022, doi:10.1021/j150576a608, 1959.

Alpert, P. A., Aller, J. Y., and Knopf, D. A.: Ice nucleation from aqueous $\mathrm{NaCl}$ droplets with and without marine diatoms, Atmos. Chem. Phys., 11, 5539-5555, doi:10.5194/acp-11-55392011, 2011.

Álvarez, V., Mattedi, S., Iglesias, M., Gonzalez-Olmos, R., and Resa, J.: Phase equilibria of binary mixtures containing methyl acetate, water, methanol or ethanol at $101.3 \mathrm{kPa}$, Phys. Chem. Liq., 49, 52-71, 2011.

Alvarez Gonzalez, J., Macedo, E., Soares, M., and Medina, A.: Liquid-liquid equilibria for ternary systems of water-phenol and solvents: data and representation with models, Fluid Phase Equilibr., 26, 289-302, 1986.

Amer, H., Paxton, R., and Winkle, M.: Methanol-ethanol-acetone, Ind. Eng. Chem., 48, 142-146, doi:10.1021/ie50553a041, 1956.

Apelblat, A. and Manzurola, E.: Solubility of oxalic, malonic, succinic, adipic, maleic, malic, citric, and tartaric acids in water from 278.15 to 338.15 K, J. Chem. Thermodyn., 19, 317-320, 1987.

Apelblat, A. and Manzurola, E.: Solubility of ascorbic, 2furancarboxylic, glutaric, pimelic, salicylic, and o-phthalic acids in water from 279.15 to $342.15 \mathrm{~K}$, and apparent molar volumes of ascorbic, glutaric, and pimelic acids in water at $298.15 \mathrm{~K}, \mathrm{~J}$. Chem. Thermodyn., 21, 1005-1008, 1989.

Arce, A., Blanco, A., Souza, P., and Vidal, I.: Liquid-liquid equilibria of the ternary mixtures water + propanoic acid + methyl ethyl ketone and water + propanoic acid + methyl propyl ketone, J. Chem. Eng. Data, 40, 225-229, doi:10.1021/je00017a047, 1995.

Arich, G. and Tagliavini, G.: Isotherme di equilibrio liquidovapore nel sistema acqua-acido acetico, La Ricerca scientifica, 28, 2493-2500, 1958.

Backes, H., Jing Jun, M. A., Bender, E., and Maurer, G.: Interfacial tensions in binary and ternary liquid-liquid systems, Chem. Eng. Sci., 45, 275-286, 1990.

Bailey, A., Harris, J., and Skau, E.: Solubilities of some normal saturated and unsaturated long-chain fatty acid methyl esters in acetone, n-Hexane, Toluene, and 1, 2-Dichloroethane, J. Chem. Eng. Data, 15, 583-585, doi:10.1021/je60047a027, 1970.

Beyer, K., Friesen, K., Bothe, J., and Palet, B.: Phase diagrams and water activities of aqueous dicarboxylic acid systems of atmospheric importance, J. Phys. Chem. A, 112, 11704-11713, doi:10.1021/jp805985t, 2008.

Blond, G., Simatos, D., Catté, M., Dussap, C., and Gros, J.: Modeling of the water-sucrose state diagram below $0{ }^{\circ} \mathrm{C}$, Carbohyd. Res., 298, 139-145, 1997.

Boese, A. B., Fink, C., Goodman, H., Hillenbrand, E., Holden, R., Jones, W., Livrngood, S., Rector, P. R., Schultze, H., Smyth, H., Tamplin, W., and Toussaint, W.: Chapters 1-16, in: Glycols, edited by: Curme, G. O. and Johnston, F., vol. 114, New York, NY: Reinhold, 389 pp., 1953.

Bomshtein, A. L. and Trofimov, A., Kudakina, E., and Serafimov, L.: Vapor-liquid phase equilibrium in binary systems formed by water, carboxylic acids $\mathrm{C}_{2}-\mathrm{C}_{4}$ and esters, Deposited Doc. Oniitekhim, 1-12, 1983.

Bonner, O. and Breazeale, W.: Osmotic and activity coefficients of some nonelectrolytes, J. Chem. Eng. Data, 10, 325-327, doi:10.1021/je60027a007, 1965.

Borisova, I., Erlykina, M., Vatskova, V., Sokolov, N., and Mikhailov, V.: Vapor-liquid equilibrium in the diethyl ether - water system at atmospheric pressure, Deposited Doc. Oniitekhim, 1-9, 1983.

Bower, V. and Robinson, R.: Isopiestic vapor pressure measurements of the ternary system: sorbitol-sodium chloride-water at $25^{\circ}$, J. Phys. Chem., 67, 1540-1541, doi:10.1021/j100801a033, 1963.

Braban, C., Carroll, M., Sarah, A., and Abbatt, J.: Phase transitions of malonic and oxalic acid aerosols, J. Phys. Chem. A, 107, 6594-6602, doi:10.1021/jp034483f, 2003.

Brown, I. and Smith, F.: Liquid-vapor equilibria VIII. The systems acetone + benzene and Acetone + carbon tetrachloride at $45^{\circ} \mathrm{C}$, Aust. J. Chem., 10, 423-428, 1957.

Cabezas, J., Arranz, J., and Berrueta, J.: Binary Vapor-liquid equilibrium of benzene-alcohol systems, Rev. Roum. Chim., 30, 903908, 1985.

Calvar, N., Dominguez, A., and Tojo, J.: Vapor-liquid equilibria for the quaternary reactive system ethyl acetate + ethanol + water + acetic acid and some of the constituent binary systems at $101.3 \mathrm{kPa}$, Fluid Phase Equilibr., 235, 215-222, 2005.

Campbell, A., Kartzmark, E., and Gieskes, J.: Vapor-liquid equilibria, densities, and refractivities in the system acetic acidchloroform-water at $25^{\circ} \mathrm{C}$, Can. J. Chem., 41, 407-429, 1963.

Campbell, S., Wilsak, R., and Thodos, G.: Vapor-liquid equilibrium measurements for the ethanol-acetone system at 372.7 , 
397.7, and 422.6 K, J. Chem. Eng. Data, 32, 357-362, doi:10.1021/je00049a021, 1987.

Carr, A. and Kropholler, H.: Vapor liquid equilibria at atmospheric pressure. binary systems of ethyl acetate-benzene, ethyl acetatetoluene, and ethyl acetate-p-xylene, J. Chem. Eng. Data, 7, 2628, doi:10.1021/je60012a007, 1962.

Carta, R. and Dernini, S.: Solubility of solid acetic acid in liquid organic solvents, J. Chem. Eng. Data, 28, 328-330, doi:10.1021/je00033a013, 1983.

Carta, R., Dernini, S., and De Santis, R.: Activity coefficients from solid-liquid and vapor-liquid equilibriums of some associated solutions, J. Chem. Eng. Data, 24, 100-103, doi:10.1021/je60081a025, 1979.

Carvoli, G. and Delogu, P.: Phase equilibria of the quaternary system acetic acid-ethylene glycol monoethyl ether acetate-ethylene glycol monoethyl ether-water, Fluid Phase Equilibr., 25, 91-105, 1986.

Chan, M., Choi, M., Ng, N., and Chan, C.: Hygroscopicity of watersoluble organic compounds in atmospheric aerosols: Amino acids and biomass burning derived organic species, Environ. Sci. Technol., 39, 1555-1562, doi:10.1021/es0495841, 2005.

Chandak, B., Nageshwar, G., and Mene, P.: Excess enthalpy, volume, and Gibbs free energy and viscosity of ethyl acetatemethyl cellosolve mixtures, J. Chem. Eng. Data, 22, 137-141, doi:10.1021/je60073a022, 1977.

Chapoy, A., Anderson, R., Haghighi, H., Edwards, T., and Tohidi, B.: Can n-propanol form hydrate?, Ind. Eng. Chem. Res., 47, 1689-1694, 2008.

Chesnokov, V.: Uber die Stabilität der Komplexe von Dimethylsulfoxyd mit Essigsäure in Anwesenheit von Carbamid, Acetamid und Aceton, Zh. Obshch. Khim., 39, 1437-1442, 1969.

Chiavone-Filho, O., Proust, P., and Rasmussen, P.: Vapor-liquid equilibria for glycol ether + water systems, J. Chem. Eng. Data, 38, 128-131, doi:10.1021/je00009a031, 1993.

Cho, T., Ochi, K., and Kojima, K.: Measurement of vapor-liquid equilibrium for systems with limited miscibility, Fluid Phase Equilibr., 11, 137-152, 1983.

Choi, J., Park, D., and Rhim, J.: Prediction of ternary Liquid-liquid equilibria using the NRTL and the UNIQUAC Models, Korean J. Chem. Eng., 3, 141-151, 1986.

Chylinski, K., Fras, Z., and Malanowski, S.: Vapor-Liquid Equilibrium in Phenol + 2-Ethoxyethanol at 363.15 to $383.15 \mathrm{~K}$, J. Chem. Eng. Data, 46, 29-33, doi:10.1021/je0001072, 2001.

Clarke, E. C. W. and Glew, D. N.: Evaluation of thermodynamic functions from equilibrium constants, T. Faraday Soc., 62, 539$547,1966$.

Clendenning, K.: Production and properties of 2,3-butanediol. XI. Evaluation of levo-2,3-butanediol as a non-volatile antifreeze compound, Can. J. Res. Sect., 24, 249-271, 1946.

Coles, K. and Popper, F.: Vapor-liquid equilibria. ethylene oxideacetaldehyde and ethylene oxide-water systems, Ind. Eng. Chem., 42, 1434-1438, doi:10.1021/ie50487a046, 1950.

Colombo, A., Battilana, P., Ragaini, V., Bianchi, C., and Carvoli, G.: Liquid-liquid equilibria of the ternary systems water + acetic acid + ethyl acetate and water + acetic acid + isophorone $(3,5$, 5-trimethyl-2-cyclohexen-1-one), J. Chem. Eng. Data, 44, 3539, doi:10.1021/je9702910, 1999.
Compernolle, S. and Müller, J.-F.: Henry's law constants of diacids and hydroxy polyacids: recommended values, Atmos. Chem. Phys., 14, 2699-2712, doi:10.5194/acp-14-2699-2014, 2014.

Compernolle, S., Ceulemans, K., and Müller, J.-F.: Influence of non-ideality on condensation to aerosol, Atmos. Chem. Phys., 9, 1325-1337, doi:10.5194/acp-9-1325-2009, 2009.

Correa, J. M., Arce, A., Blanco, A., and Correa, A.: Liquid-liquid equilibria of the system water + acetic acid + methyl ethyl ketone at several temperatures, Fluid Phase Equilibr., 32, 151-162, 1987.

Dakshinamurty, P., Rao, G. J., and Rao, C. V.: Vapour-liquid equilibria in the system water-propionic acid, J. Appl. Chem., 11, 226-228, 1961.

Dallos, A., Laszlo-Parragi, M., Ratkovics, F., Hahn, G., Kalali, H., and Kohler, F.: The thermodynamics of the system acetic acid 2-butanone, Z. Chem., 26, 35-36, 1986.

Danciu, E.: Echilibrul lichid-vapori. IV. Binarele sistemului ternar: propenoxid - aldehida propionica - acetona in condetii izobare, Rev. Chim. Bucharest., 21, 149-157, 1970.

d'Avila, S. and Silva, R.: Isothermal vapor-liquid equilibrium data by total pressure method. systems acetaldehyde-ethanol, acetaldehyde-water, and ethanol-water, J. Chem. Eng. Data, 15, 421-424, doi:10.1021/je60046a010, 1970.

de Leeuw, H.: About the system acetaldehyde - ethanol, Z. Phys. Chem. Stoch. Ve., 77, 284-314, 1911.

De Oliveira, L. and Aznar, M.: (Liquid + liquid) equilibrium of $\{$ water + phenol + (1-butanol, or 2-butanol, or tert-butanol) $\}$ systems, J. Chem. Thermodyn., 42, 1379-1385, 2010.

Dean, J. A.: Langes Handbook of Chemistry, 15th Edn., McGrawHill Companies, Inc., New York, USA, 6.1-6.158, 1999.

Domalski, E. S. and Hearing, E. D.: Heat capacities and entropies of organic compounds in the condensed phase. volume III, J. Phys. Chem. Ref. Data, 25, 523 pp. 1996.

Dománska, U., Morawski, P., and Piekarska, M.: Solid-liquid phase equilibria of 1-decanol and 1-dodecanol with fragrance raw materials based on cyclohexane, J. Chem. Eng. Data, 54, 12711276, 2009.

Dykyj, J., V., Kuska, V., and Seprakova, M.: Physical properties of ethylene glycol and its derivatives. I. Solidification points of solutions of ethylene glycols, Chem. Zvesti, 10, 193-203, 1956.

Dykyj, J., Svoboda, J., Wilhoit, R. C., Frenkel, M., and Hall, K. R.: Organic compounds, $\mathrm{C}_{1}$ to $\mathrm{C}_{57}$. Part 1., in: Landolt-Börnstein - Group IV Physical Chemistry Numerical Data and Functional Relationships in Science and Technology, edited by: Hall, K. R., vol. 20B, Vapor Pressure and Antoine Constants for Oxygen Containing Organic Compounds, SpringerMaterials - The Landolt-Börnstein Database, 14-110, doi:10.1007/10688583_3, 2000.

Escobedo-Alvarado, G. and Sandler, S.: Vapor-liquid equilibrium of two aqueous systems that exhibit liquid-liquid phase separation, J. Chem. Eng. Data, 44, 319-322, doi:10.1021/je980228q, 1999.

Esquível, M. and Bernardo-Gil, M.: Liquid-liquid equilibria for the systems water-alcohols-acetic acid, Fluid Phase Equilibr., 57, 307-316, 1990.

Fandary, M., Aljimaz, A., Al-Kandary, J., and Fahim, M.: Liquid-liquid equilibria for the system water + ethanol + ethyl tert-butyl ether, J. Chem. Eng. Data, 44, 1129-1131, doi:10.1021/je980253w, 1999. 
Fang, W., Liu, G., Wang, L., Zhang, X., Mi, Z., Zhang, S., and Yin, Y.: Liquid-liquid equilibria for the ternary system water + methyl isobutyl ketone + tert-butyl alcohol at several temperatures, J. Chem. Eng. Data, 53, 466-470, doi:10.1021/je700554u, 2008.

Faucon, M.: Recherches sur les melanges d'eau et d'acides gras, Ann. Chim. Phys., 19, 70-152, 1910.

Ferino, I., Marongiu, B., Monaci, R., Solinas, V., and Torrazza, S.: Thermodynamic properties of aqueous non-electrolyte mixtures. enthalpy of mixing and liquid-liquid equilibrium of water + aliphatic aldehyde mixtures, Thermochim. Acta, 65, 157168, 1983.

Fernández-Torres, M., Gomis-Yagües, V., Ramos-Nofuentes, M., and Ruiz-Beviá, F.: The influence of the temperature on the liquid-liquid equilibrium of the ternary system 1-pentanolethanol-water, Fluid Phase Equilibr., 164, 267-273, 1999.

Fiege, C., Joh, R., Petri, M., and Gmehling, J.: Solid-liquid equilibria for different heptanones with benzene, cyclohexane, and ethanol, J. Chem. Eng. Data, 41, 1431-1433, doi:10.1021/je960140h, 1996.

Fredenslund, A., Jones, R. L., and Prausnitz, J. M.: Groupcontribution estimation of activity coefficients in nonideal liquid mixtures, AIChE J., 21, 1086-1099, 1975.

Fu, H., Cheng, G., and Han, S.: Vapor-liquid equilibrium for the systems containing association component. Ii. Acetic acid and water; acetic acid and 2-butanone; acetic acid and 2-pentanone; acetic acid and acetic acid propyl ester, Huaxue Gongcheng, 6, 56-61, 1986.

Fu, H., Mo, X., Han, S., and Li, H.: Study on isothermal vaporliquid equilibrium for ethanol-benzene, chloroform-benzene and ethanol-chhloroform binary systems, Shiyou Xuebao Shiyou Jiagong, 11, 87-92, 1995.

Ganbavale, G., Marcolli, C., Krieger, U. K., Zuend, A., Stratmann, G., and Peter, T.: Experimental determination of the temperature dependence of water activities for a selection of aqueous organic solutions, Atmos. Chem. Phys., 14, 9993-10012, doi:10.5194/acp-14-9993-2014, 2014.

Gaube, J., Hammer, S., and Pfennig, A.: A new equilibrium cell with variable cell volume for static vapour-pressure measurements, Fluid Phase Equilibr., 123, 245-257, 1996.

Gilburd, M., Yurkevich, B., and Polytanskaya, T.: Liquid-vapor phase equilibrium in an acetaldehyde-propylene oxide system, J. Appl. Chem.-USSR, 52, 2247-2249, 1979.

Gilburd, M., Politanskaya, T., and Yurkevich, B.: Liquid-vapor phase equilibrium in the systems ethyl acetate-acetone-allyl alcohol, and ethyl acetate-acetic acid under reduced pressure, J. Appl. Chem.-USSR, 54, 938-940, 1981.

Gmehling, J.: From UNIFAC to modified UNIFAC to PSRK with the help of DDB, Fluid Phase Equilibr., 107, 1-29, 1995.

Gmehling, J.: Potential of thermodynamic tools (group contribution methods, factual data banks) for the development of chemical processes, Fluid Phase Equilibr., 210, 161-173, 2003.

Gmehling, J.: Present status and potential of group contribution methods for process development, J. Chem. Thermodyn., 41, 731-747, 2009.

Gmehling, J. and Onken, U.: Vapor-Liquid Equilibrium Data Collection: Volume I, Part 1-Aqueous-Organic Systems, Chemistry Data Series, Frankfurt/Main: DECHEMA Deutsche Gesellschaft für Chemisches Apparatewesen, 1977.
Gmehling, J. and Onken, U.: Vapor-Liquid Equilibrium Data Collection: Volume I, Part 1c(in conjunction with part 1d)Aqueous Systems (Supplement 3), Chemistry Data Series, Frankfurt/Main: DECHEMA Deutsche Gesellschaft für Chemisches Apparatewesen, 2003a.

Gmehling, J. and Onken, U.: Vapor-Liquid Equilibrium Data Collection: Volume I, Part 1d(in conjunction with part 1c)Aqueous Systems (Supplement 4), Chemistry Data Series, Frankfurt/Main: DECHEMA Deutsche Gesellschaft für Chemisches Apparatewesen, 2003b.

Gmehling, J., Onken, U., and Arlt, W.: Vapor-Liquid Equilibrium Data Collection: Volume I, Part 1a-Aqueous-Organic Systems(Supplement 1), Chemistry Data Series, Frankfurt/Main: DECHEMA Deutsche Gesellschaft für Chemisches Apparatewesen, 1981.

Gmehling, J., Onken, U., and Rarey-Nies, J. R.: Vapor-Liquid Equilibrium Data Collection: Volume I, Part 1b-Aqueous Systems (Supplement 2), Chemistry Data Series, Frankfurt/Main: DECHEMA Deutsche Gesellschaft für Chemisches Apparatewesen, 1988.

Gmehling, J., Li, J., and Schiller, M.: A modified UNIFAC model. 2. Present parameter matrix and results for different thermodynamic properties, Ind. Eng. Chem. Res., 32, 178-193, doi:10.1021/ie00013a024, 1993.

Gmehling, J., Lohmann, J., Jakob, A., Li, J., and Joh, R.: A modified UNIFAC (Dortmund) model. 3. Revision and extension, Ind. Eng. Chem. Res., 37, 4876-4882, doi:10.1021/ie980347z, 1998.

Gmehling, J., Wittig, R., Lohmann, J., and Joh, R.: A modified UNIFAC (Dortmund) model. 4. Revision and extension, Ind. Eng. Chem. Res., 41, 1678-1688, doi:10.1021/ie0108043, 2002.

Gmehling, J., Kolbe, B., Kleiber, M., and Rarey, J.: Chemical Thermodynamics for Process Simulation, Wiley-VCH, Weinheim, 2012.

Gomis-Yagües, V., Ruíz-Beviá, F., Ramos-Nofuentes, M., and Fernández-Torres, M.: The influence of the temperature on the liquid-liquid equilibrium of the ternary system 1-butanol-1propanol-water, Fluid Phase Equilibr., 149, 139-145, 1998.

Gonzalez, E.: Vapor-liquid equilibria at $101.32 \mathrm{kPa}$ mixtures of butyl esters and propan-2-ol., J. Chem. Eng. Jpn., 29, 294-299, 1996.

Griswold, J. and Wong, S.: Phase-equilibria of the actone-methanolwater system from $100^{\circ} \mathrm{C}$ into the critical region, Chem. Eng. Symp. Ser., 48, 18-34, 1952.

Hallquist, M., Wenger, J. C., Baltensperger, U., Rudich, Y., Simpson, D., Claeys, M., Dommen, J., Donahue, N. M., George, C., Goldstein, A. H., Hamilton, J. F., Herrmann, H., Hoffmann, T., Iinuma, Y., Jang, M., Jenkin, M. E., Jimenez, J. L., Kiendler-Scharr, A., Maenhaut, W., McFiggans, G., Mentel, Th. F., Monod, A., Prévôt, A. S. H., Seinfeld, J. H., Surratt, J. D., Szmigielski, R., and Wildt, J.: The formation, properties and impact of secondary organic aerosol: current and emerging issues, Atmos. Chem. Phys., 9, 5155-5236, doi:10.5194/acp-9-51552009, 2009.

Hansen, H. K., Rasmussen, P., Fredenslund, A., Schiller, M., and Gmehling, J.: Vapor-liquid-equilibria by UNIFAC group contribution. 5. Revision and extension, Ind. Eng. Chem. Res., 30, 2352-2355, 1991.

Haughton, C.: V-I equilbria of benzene and acetic acid, Br. Chem. Eng., 12, 1102-1103, 1967. 
Hill, A.: The mutual solubility of liquids. I. The mutual solubility of ethyl ether and water. II. The solubility of water in benzene, J. Am. Chem. Soc., 45, 1143-1155, doi:10.1021/ja01658a007, 1923.

Hirata, M. and Hoshino, D.: A study on vapor-liquid equilibria based on the theory of solution of groups model, Asahi Garasu Kogyo Gijutsu Shoreikai Kenkyu Hokoku, 41, 115-122, 1982.

Hirata, M., Ohe, S., and Nagahama, K.: Computer Aided Data Book of Vapor-Liquid Equilibria, Elsevier Science \& Technology, Elsevier Scientific Publishing Comp., Tokio-Amsterdam-OxfordNew York, 933 pp., 1975.

Ito, T. and Yoshida, F.: Vapor-liquid equilibria of water-lower fatty acid systems: water-formic acid, water acetic acid and waterpropionic acid, J. Chem. Eng. Data, 8, 315-320, 1963.

Jacobson, M., Hansson, H., Noone, K., and Charlson, R.: Organic atmospheric aerosols: review and state of the science, Rev. Geophys., 38, 267-294, doi:10.1029/1998RG000045, 2000.

Jakob, A.: DETHERM database, DECHEMA e.V. (unpublished data), Universität Oldenburg, 1994.

Jakob, A., Grensemann, H., Lohmann, J., and Gmehling, J.: Further development of modified UNIFAC (Dortmund): revision and extension 5, Ind. Eng. Chem. Res., 45, 7924-7933, doi:10.1021/ie060355c, 2006.

Jaoui, M., Achard, C., and Rogalski, M.: Solubility as a function of temperature of selected chlorophenols and nitrophenols in aqueous solutions containing electrolytes or surfactants, J. Chem. Eng. Data, 47, 297-303, doi:10.1021/je0102309, 2002.

Kanno, H., Miyata, K., Tomizawa, K., and Tanaka, H.: Additivity rule holds in supercooling of aqueous solutions, J. Phys. Chem. A, 108, 6079-6082, doi:10.1021/jp048676u, 2004.

Kanno, H., Soga, M., and Kajiwara, K.: Linear relation between $T_{H}$ (homogeneous ice nucleation temperature) and $T_{\mathrm{m}}$ (melting temperature) for aqueous solutions of sucrose, trehalose, and maltose, Chem. Phys. Lett., 443, 280-283, 2007.

Keyes, D.: Liquid-vapor composition curves of acetic acid and water at subatmospheric pressures, Ind. Eng. Chem., 25, 569-569, doi:10.1021/ie50281a026, 1933.

Kim, J. and Park, D.: Liquid-liquid equilibrium for the ternary systems of solvents + water + propionic acid at $25^{\circ} \mathrm{C}$ and atmospheric pressure, Korean J. Chem. Eng., 22, 256-263, 2005.

Kliment, V., Fried, V., and Pick, J.: Gleichgewicht Flüssigkeitdampf XXXII I.Systeme Butylacetat-Phenol und wasser-Phenol, Collect. Czech. Chem. C., 29, 2008-2015, 1964.

Knight, W. S.: Thermodynamics of Aqueous Solutions of Alcohols and P-dioxane, PhD Thesis, Princeton University, 179 pp., 1962.

Knopf, D. and Rigg, Y.: Homogeneous ice nucleation from aqueous inorganic/organic particles representative of biomass burning: water activity, freezing temperatures, nucleation rates, J. Phys. Chem. A, 115, 762-773, doi:10.1021/jp109171g, 2011.

Koga, Y., Tanaka, T., Atake, T., Westh, P., and Hvidt, A.: Mixing schemes and liquid-solid phase diagram in the water-rich region of aqueous 2-butoxyethanol., B. Chem. Soc. Jpn., 67, 23932397, 1994.

Kolb, D.: Solubilities of fatty acids in selected organic solvents at low temperatures, Diss. Abstr., 20, 82-86, 1959.

Koop, T.: Homogeneous ice nucleation in water and aqueous solutions, Z. Phys. Chem., 218, 1231-1258, doi:10.1524/zpch.218.11.1231.50812, 2004.
Koop, T. and Zobrist, B.: Parameterizations for ice nucleation in biological and atmospheric systems, Phys. Chem. Chem. Phys., 11, 10839-10850, 2009.

Koop, T., Luo, B., Tsias, A., and Peter, T.: Water activity as the determinant for homogeneous ice nucleation in aqueous solutions, Nature, 406, 611-614, doi:10.1038/35020537, 2000.

Koop, T., Bookhold, J., Shiraiwa, M., and Pöschl, U.: Glass transition and phase state of organic compounds: dependency on molecular properties and implications for secondary organic aerosols in the atmosphere, Phys. Chem. Chem. Phys., 13, 19238-19255, doi:10.1039/C1CP22617G, 2011.

Krieger, U. K., Marcolli, C., and Reid, J. P.: Exploring the complexity of aerosol particle properties and processes using single particle techniques, Chem. Soc. Rev., 41, 6631-6662, 2012.

Kurihara, K., Hori, H., and Kojima, K.: Vapor-liquid equilibrium data for acetone + methanol + benzene, chloroform + methanol + benzene, and constituent binary systems at $101.3 \mathrm{kPa}$, J. Chem. Eng. Data, 43, 264-268, doi:10.1021/je970231u, 1998.

Lalande, A.: Equilibres entre Phases Condensèes dans le système eau-alcool-ether, J. Chim. Phys. PCB, 31, 583-609, 1934.

Larsen, B., Rasmussen, P., and Fredenslund, A.: A modified UNIFAC group-contribution model for prediction of phase equilibria and heats of mixing, Ind. Eng. Chem. Res., 26, 2274-2286, doi:10.1021/ie00071a018, 1987.

Lee, M. and $\mathrm{Hu}, \mathrm{C}$. .: Isothermal vapor-liquid equilibria for mixtures of ethanol, acetone, and diisopropyl ether, Fluid Phase Equilibr., 109, 83-98, 1995.

Lerici, C., Piva, M., and ROSA, M.: Water activity and freezing point depression of aqueous solutions and liquid foods, J. Food Sci., 48, 1667-1669, 2006.

Letcher, T., Redhi, G., Radloff, S., and Domanska, U.: Liquidliquid equilibria for mixtures of butanal + an alkanol + water at 298.15 K, J. Chem. Eng. Data, 41, 707-712, 1996.

Leu, A., Chen, C., and Robinson, D.: Vapor-liquid equilibrium in selected binary systems, AIChE Sym. S., 85, 11-16, 1989.

Lienhard, D., Bones, D., Zuend, A., Krieger, U., Reid, J., and Peter, T.: Measurements of thermodynamic and optical properties of selected aqueous organic and organic-inorganic mixtures of atmospheric relevance, J. Phys. Chem. A, 116, 9954-9968, doi:10.1021/jp3055872, 2012.

Lihua, F., Peisheng, M., and Zhengle, X.: Measurement and correlation for solubility of adipic acid in several solvents, Chinese J. Chem. Eng., 15, 110-114, 2007.

Lin, C., Qing, A., and Feng, Q.: A new differential mutation base generator for differential evolution, J. Global Optim., 49, 69-90, 2011.

Lin, H., Tien, H., Hone, Y., and Lee, M.: Solubility of selected dibasic carboxylic acids in water, in ionic liquid of $\left[\mathrm{B}_{\operatorname{mim}}\right]\left[\mathrm{BF}_{4}\right]$, and in aqueous $\left[\mathrm{B}_{\text {mim }}\right]\left[\mathrm{BF}_{4}\right]$ solutions, Fluid Phase Equilibr., 253, 130-136, 2007.

Lintomen, L., Pinto, R., Batista, E., Meirelles, A., and Maciel, M.: Liquid-liquid equilibrium of the water + citric acid + short chain alcohol + tricaprylin system at 298.15 K, J. Chem. Eng. Data, 46, 546-550, doi:10.1021/je000226h, 2001.

Loehe, J. R., Van Ness, H. C., and Abbott, M. M.: Vapor/liquid/liquid equilibrium. Total-pressure data and GE for water/methyl acetate at $50{ }^{\circ} \mathrm{C}$, J. Chem. Eng. Data, 28, 405-407, doi:10.1021/je00034a017, 1983. 
Lohmann, J., Joh, R., and Gmehling, J.: Solid-liquid equilibria of viscous binary mixtures with alcohols, J. Chem. Eng. Data, 42, 1170-1175, doi:10.1021/je9700683, 1997.

Lohmann, J., Joh, R., and Gmehling, J.: From UNIFAC to modified UNIFAC (Dortmund), Ind. Eng. Chem. Res., 40, 957-964, doi:10.1021/ie0005710, 2001.

Lohmann, U. and Feichter, J.: Global indirect aerosol effects: a review, Atmos. Chem. Phys., 5, 715-737, doi:10.5194/acp-5-7152005, 2005.

Maffia, M. and Meirelles, A.: Water activity and $\mathrm{pH}$ in aqueous polycarboxylic acid systems, J. Chem. Eng. Data, 46, 582-587, doi:10.1021/je0002890, 2001.

Mafra, M. and Krähenbühl, M.: Liquid-liquid equilibrium of (water + acetone) with cumene or $\alpha$-methylstyrene or phenol at temperatures of (323.15 and 333.15) K, J. Chem. Eng. Data, 51, $753-$ 756, 2006

Marcolli, C. and Krieger, U. K.: Phase changes during hygroscopic cycles of mixed organic/inorganic model systems of tropospheric aerosols, J. Phys. Chem. A, 110, 1881-1893, doi:10.1021/jp0556759, 2006.

Marcolli, C. and Peter, Th.: Water activity in polyol/water systems: new UNIFAC parameterization, Atmos. Chem. Phys., 5, 15451555, doi:10.5194/acp-5-1545-2005, 2005.

Marcolli, C., Luo, B. P., Peter, Th., and Wienhold, F. G.: Internal mixing of the organic aerosol by gas phase diffusion of semivolatile organic compounds, Atmos. Chem. Phys., 4, 25932599, doi:10.5194/acp-4-2593-2004, 2004.

Martin, M., Cocero, M., and Mato, R.: Vapor-liquid equilibrium data at $298.15 \mathrm{~K}$ for binary systems containing methyl acetate or methanol with 2-methoxyethanol or 2-ethoxyethanol, J. Chem. Eng. Data, 39, 535-537, doi:10.1021/je00015a030, 1994.

Martínez, N., Lladosa, E., Burguet, M., and Montón, J.: Isobaric vapour-liquid equilibria for binary systems of 2-butanone with ethanol, 1-propanol, and 2-propanol at 20 and $101.3 \mathrm{kPa}$, Fluid Phase Equilibr., 270, 62-68, 2008.

May, W., Wasik, S., Miller, M., Tewari, Y., Brown-Thomas, J., and Goldberg, R.: Solution thermodynamics of some slightly soluble hydrocarbons in water, J. Chem. Eng. Data, 28, 197-200, doi:10.1021/je00032a021, 1983.

Meehan, G. and Murphy, N.: A new correlation for binary systems with an associating component, Chem. Eng. Sci., 20, 757-769, 1965.

Mejía, A., Segura, H., Cartes, M., Cifuentes, L., and Flores, M.: Phase equilibria and interfacial tensions in the systems methyl tert-butyl ether + acetone + cyclohexane, methyl tertbutyl ether + acetone and methyl tert-butyl ether + cyclohexane, Fluid Phase Equilibr., 273, 68-77, 2008.

Mertl, I.: Liquid-vapor equilibrium. IL. Phase equilibria in the ternary system ethyl acetate-ethanol-water, Collect. Czech. Chem. C., 37, 366-374, 1972.

Miao, X., Zhang, H., Wang, T., and He, M.: Liquid-liquid equilibria of the ternary system water + acetic acid + methyl tert-butyl ether, J. Chem. Eng. Data, 52, 789-793, doi:10.1021/je060409p, 2007.

Miyamoto, S., Nakamura, S., Iwai, Y., and Arai, Y.: Measurement of isothermal vapor-liquid equilibria for hydrocarbon + monocarboxylic acid binary systems by a flow-type apparatus, J. Chem. Eng. Data, 45, 857-861, doi:10.1021/je000015c, 2000 .
Miyamoto, S., Nakamura, S., Iwai, Y., and Arai, Y.: Measurement of isothermal vapor-liquid equilibria for binary and ternary systems containing monocarboxylic acid, J. Chem. Eng. Data, 46, 12251230, doi:10.1021/je0003849, 2001.

Miyata, K. and Kanno, H.: Supercooling behavior of aqueous solutions of alcohols and saccharides, J. Mol. Liq., 119, 189-193, 2005.

Moeller, W., Englund, S., Tsui, T. K., and Othmer, D.: Compositions of vapors from boiling solutions - equilibria under pressure of systems: ethyl ether-ethyl alcohol and ethyl ether-water-ethyl alcohol, Ind. Eng. Chem., 43, 711-717, doi:10.1021/ie50495a039, 1951.

Montón, J., Munoz, R., Burguet, M., and Torre, J.: Isobaric vapor-liquid equilibria for the binary systems isobutyl alcohol + isobutyl acetate and tert-butyl alcohol + tert-butyl acetate at 20 and 101.3 kPa, Fluid Phase Equilibr., 227, 19-25, 2005.

Mozzhukhin, A., Mitropolśkaya, V., Serafimov, L., and Torubarov, A.: Liquid-vapor phase equilibrium in binary mixtures of some oxygen-containing products at $760 \mathrm{~mm} \mathrm{Hg}$, Zh. Fiz. Khim., 41, 116-119, 1967.

Mun, S. and Lee, H.: Vapor-liquid equilibria of the water +1 , 3-propanediol and water +1 , 3-propanediol + lithium bromide systems, J. Chem. Eng. Data, 44, 1231-1234, 1999.

Murphy, D., Cziczo, D., Froyd, K., Hudson, P., Matthew, B., Middlebrook, A., Peltier, R., Sullivan, A., Thomson, D., and Weber, R.: Single-particle mass spectrometry of tropospheric aerosol particles, J. Geophys. Res., 111, D23S32, doi:10.1029/2006JD007340, 2006.

Narayana, A., Naik, S., and Rath, P.: Salt effect in isobaric vaporliquid equilibria of acetic acid-water system, J. Chem. Eng. Data, 30, 483-485, doi:10.1021/je00042a035, 1985.

Naumann, D. and Wagner, H.: Vapour-liquid equilibria of several mixtures containing 2-butanone, 2-butenal, ethoxyethanol, and toluene at $330.15 \mathrm{~K}, \mathrm{~J}$. Chem. Thermodyn., 18, 81-87, 1986.

Negadi, L., Wilken, M., and Gmehling, J.: Solid-liquid equilibria for binary organic systems containing 1-methoxy-2-propanol and 2-butoxy ethanol, J. Chem. Eng. Data, 51, 1873-1876, 2006.

Nelder, J. A. and Mead, R.: A simplex method for function minimization, Comput. J., 7, 308-313, 1965.

Newman, M., Hayworth, C., and Treybal, R.: Dehydration of aqueous methyl ethyl ketone-equilibrium data for extractive distillation and solvent extraction, J. Chem. Eng. Data, 41, 2039-2043, doi:10.1021/je00009a031, 1949.

Ninni, L. Camargo, M. S., and Meirelles, A. J. A.: Water activity in poly(ethylene glycol) aqueous solutions, Thermochim. Acta, 328, 169-176, 1999.

Oh, J. and Park, S.: Isothermal vapor-liquid equilibria at $333.15 \mathrm{~K}$ and excess molar volumes at $298.15 \mathrm{~K}$ of ethyl tert-butyl ether $($ ETBE $)+$ alcoh-1-ol $\left(\mathrm{C}_{1}-\mathrm{C}_{4}\right)$ mixtures, J. Chem. Eng. Data, 43, 1009-1013, doi:10.1021/je980089c, 1998.

Ohta, T., Koyabu, J., and Nagata, I.: Vapor-liquid equilibria for the ternary ethanol-2-butanone-benzene system at 298.15 K, Fluid Phase Equilibr., 7, 65-73, 1981.

Okamoto, B., Wood, R., and Thompson, P.: Freezing points of aqueous alcohols. free energy of interaction of the $\mathrm{CHOH}, \mathrm{CH}_{2}$, $\mathrm{CONH}$ and $\mathrm{C}=\mathrm{C}$ functional groups in dilute aqueous solutions, $\mathrm{J}$. Chem. Soc. Faraday T., 74, 1990-2007, 1978. 
Olsen, J., Brunjes, A., and Olsen, J.: Freezing and flow points for glycerol, prestone, denatured alcohol, and methanol, Ind. Eng. Chem., 22, 1315-1317, 1930.

Omar, W. and Ulrich, J.: Solid liquid equilibrium, metastable zone, and nucleation parameters of the oxalic acid-water system, Cryst. Growth Des., 6, 1927-1930, doi:10.1021/cg060112n, 2006.

Othmer, D.: Composition of vapors from boiling binary solutions, Ind. Eng. Chem., 35, 614-620, doi:10.1021/ie50401a018, 1943.

Othmer, D., Chudgar, M., and Levy, S.: Binary and ternary systems of acetone, methyl ethyl ketone, and water, Ind. Eng. Chem., 44, 1872-1881, doi:10.1021/ie50512a042, 1952.

Othmer, D. F. and Benenati, R.: Composition of vapors from boiling binary solutions, Ind. Eng. Chem., 37, 299-303, doi:10.1021/ie50423a024, 1945.

Ott, J., Goates, J., and Lamb, J.: Solid-liquid phase equilibria in water + ethylene glycol, J. Chem. Thermodyn., 4, 123-126, 1972.

Pankow, J.: Gas/particle partitioning of neutral and ionizing compounds to single and multi-phase aerosol particles. 1. Unified modeling framework, Atmos. Environ., 37, 3323-3333, 2003.

Park, S., Han, K., and Gmehling, J.: Vapor-liquid equilibria and excess properties for methyl tert-butyl ether (MTBE) containing binary systems, Fluid Phase Equilibr., 200, 399-409, 2002.

Paterno, E. and Ampola, G.: Sul massimo abassamento nella temperatura di congelamento dei miscugli, Gazz. Chim. Ital., 27, 481-536, 1897.

Peng, C., Chan, M. N., and Chan, C. K.: The hygroscopic properties of dicarboxylic and multifunctional acids: measurements and UNIFAC predictions, Environ. Sci. Technol., 35, 4495-4501, doi:10.1021/es0107531, 2001.

Perrakis, N.: The physical properties of liquid double mixtures in the neighborhood of the critical state of miscibility, J. Chim. Phys., 22, 280-305, 1925.

Pickering, S.: LXXI. A study of the properties of some strong solutions, J. Chem. Soc., 63, 998-1027, 1893.

Powell, M.: The BOBYQA algorithm for bound constrained optimization without derivatives, Cambridge NA Report NA2009/06, University of Cambridge, Cambridge, 39 pp., 2009.

Prausnitz, J., Lichtenthaler, R., and de Azevedo, E.: Molecular Thermodynamics of Fluid-phase Equilibria, 2nd edn., PrenticeHall Inc., Englewood Cliffs, New Jersey, USA, 600 pp., 1986.

Proust, P. and Fernandez, J.: Experimental solid-liquid equilibria of binary mixtures of organic compounds, Fluid Phase Equilibr., 29, 265-272, 1986.

Pushin, N. and Glagoleva, A.: CCCXXXVI I. The equilibrium in systems composed of water and alcohols: methyl alcohol, pinacone, glycerol, and erythritol, J. Chem. Soc., 121, 2813-2822, 1922.

Raatikainen, T. and Laaksonen, A.: Application of several activity coefficient models to water-organic-electrolyte aerosols of atmospheric interest, Atmos. Chem. Phys., 5, 2475-2495, doi:10.5194/acp-5-2475-2005, 2005.

Radnai, G., Rasmussen, P., and Fredenslund, A.: Vapor-liquid equilibrium data for binary mixtures contianing aldehydes and esters and for the mixture 1,1,2 tricholoro-1,2,2 trifluoroethane plus nhexane, AIChE Sym. S., 83, 70-79, 1987.

Rarey, J., Horstmann, S., and Gmehling, J.: Vapor-liquid equilibria and vapor pressure data for the systems ethyl tert-butyl ether + ethanol and ethyl tert-butyl ether + water, J. Chem. Eng. Data, 44, 532-538, doi:10.1021/je980229i, 1999.
Rasmussen, P. and Kierkegaard, L., and Fredenslund, A.: Vaporliquid equilibria, in: Ketone - Organic Acid Mixtures, Chemical Engineering with Per Soltoft, Copenhagen, Frankfurt/Main: DECHEMA Deutsche Gesellschaft für Chemisches Apparatewesen, 129-139, 1977.

Reichl, A., Daiminger, U., Schmidt, A., Davies, M., Hoffmann, U., Brinkmeier, C., Reder, C., and Marquardt, W.: A non-recycle flow still for the experimental determination of vapor-liquid equilibria in reactive systems, Fluid Phase Equilibr., 153, 113134, 1998

Rius, A., Otero, J., and Macarron, A.: Equilibres liquide-vapeur de mélanges binaires donnant une réaction chimique: systémes méthanol-acide acétique; éthanol-acide acétique; n-propanolacide acétique; n-butanol-acide acétique, Chem. Eng. Sci., 10, 105-111, doi:10.1016/0009-2509(59)80029-4, 1959.

Roloff, M.: Beiträge zur Kenntnis der Kryohydrate, Z. Phys. Chem.Leipzig, 17, 325-356, 1895.

Ross, H.: Cryoscopic studies-concentrated solutions of hydroxy compounds, Ind. Eng. Chem., 46, 601-610, doi:10.1021/ie50531a054, 1954.

Ruegg, M. and Blanc, B.: The water activity of honey and related sugar solutions, Lebensm. Wiss. Technol., 14, 1-6, 1981.

Ruiz Bevia, F., Prats Rico, D., Gomis Yagües, V., and Varo Galvañ, P.: Quaternary liquid-liquid equilibrium: water-acetic acid1-butanol-n-butyl acetate at $25^{\circ} \mathrm{C}$, Fluid Phase Equilibr., 18, 171-183, 1984.

Sanz, M., Blanco, B., Beltrán, S., Cabezas, J., and Coca, J.: Vapor liquid equilibria of binary and ternary systems with water, 1, 3-propanediol, and glycerol, J. Chem. Eng. Data, 46, 635-639, 2001.

Sapgir, S.: Studies on the theory of concentrated solutions. VI I. Application of thermal analysis to binary mixtures consisting of organic substances with very low melting points, B. Soc. Chim. Belg., 38, 392-408, 1929.

Saxena, P. and Hildemann, L. M.: Water absorption by organics: survey of laboratory evidence and evaluation of UNIFAC for estimating water activity, Environ. Sci. Technol., 31, 3318-3324, 1997.

Scatchard, G. and Wilson, G.: Vapor-liquid equilibrium. XIII. the system water-butyl glycol from 5 to $85^{\circ}$, J. Am. Chem. Soc., 86, 133-137, doi:10.1021/ja01056a004, 1964.

Schneider, G. and Wilhelm, G.: Vapor-liquid equilibrium of the system water - 2-butoxyethanol, Z. Phys. Chem. Neue Fol., 20, 219-232, 1959.

Schreinemakers, F.: Dampfdrucke binärer und ternärer Gemische, Z. Phys. Chem., 35, 459-479, 1900.

Sebastiani, E. and Lacquaniti, L.: Acetic acid-water system thermodynamical correlation of vapor-liquid equilibrium data, Chem Eng. Sci., 22, 1155-1162, 1967.

Sei, T. and Gonda, T.: Melting point of ice in aqueous saccharide solutions, J. Cryst. Growth, 293, 110-112, 2006.

Senol, A.: Phase equilibria for ternary liquid systems of (water + carboxylic acid or alcohol +1 -hexanol) at $T=293.15 \mathrm{~K}$ : modelling considerations, J. Chem. Thermodyn., 36, 1007-1014, 2004.

Shalmashi, A. and Eliassi, A.: Solubility of salicylic acid in water, ethanol, carbon tetrachloride, ethyl acetate, and xylene, J. Chem. Eng. Data, 53, 199-200, 2008. 
Shanghai-Inst. and Zhejiang, U.: Measurement of vapor-liquid equilibrium for the systems in the industrial preparation of acetic acid (I), Huaxue.Gongcheng, 1, 75-85, 1978.

Shiraiwa, M., Zuend, A., Bertram, A., and Seinfeld, J. H.: Gasparticle partitioning of atmospheric aerosols: interplay of physical state, non-ideal mixing and morphology, Phys. Chem. Chem. Phys., 15, 11441-11453, doi:10.1039/C3CP51595H, 2013.

Sólimo, H., Bonatti, C., Zurita, J., and Gramajo de Doz, M.: Liquidliquid equilibria for the system water + propionic acid +1butanol at $303.2 \mathrm{~K}$. effect of addition of sodium chloride, Fluid Phase Equilibr., 137, 163-172, 1997.

Song, M., Marcolli, C., Krieger, U. K., Zuend, A., and Peter, T.: Liquid-liquid phase separation and morphology of internally mixed dicarboxylic acids/ammonium sulfate/water particles, Atmos. Chem. Phys., 12, 2691-2712, doi:10.5194/acp-12-26912012, 2012.

Soujanya, J., Satyavathi, B., and Vittal Prasad, T.: Experimental (vapour + liquid) equilibrium data of (methanol + water), (water + glycerol) and (methanol + glycerol) systems at atmospheric and sub-atmospheric pressures, J. Chem. Thermodyn., 42, 621624, 2010.

Stephenson, R.: Mutual solubilities: water-ketones, water-ethers, and water-gasoline-alcohols, J. Chem. Eng. Data, 37, 80-95, doi:10.1021/je00005a024, 1992.

Stephenson, R. and Stuart, J.: Mutual binary solubilities: wateralcohols and water-esters, J. Chem. Eng. Data, 31, 56-70, doi:10.1021/je00043a019, 1986.

Subrahmanyam, V. and Murty, P.: Vapour-liquid equilibria: systems:(1) acetone-ethyl acetate;(2) acetone-n-propyl acetate, J. Appl. Chem., 14, 500-502, doi:10.1002/jctb.5010141106, 1964.

Suska, J.: Phase-equilibria in binary-systems containing acetaldehyde, Collect. Czech. Chem. C., 44, 1852-1856, 1979.

Takahashi, S., Otake, K., Takahashi, T., and Iguchi, A.: Liquidliquid equilibria of phenol-water-solvent systems, Kagaku Kogaku Ronbunshu, 14, 531-535, 1988.

Tapper, M., Engelhardt, C., Schecter, B. A., Fried, V., and Zudkevitch, D.: Vapor-liquid equilibrium and liquid-liquid equilibrium of the n-butanal-water system, AIChE Sym. S., 81, 136$141,1985$.

Tarasenkov, D.: Freezing temperature of mixtures of benzene with toluene, alcohol and gasoline, Zh. Prikl. Khim., 3, 153-155, 1930.

Thorat, R. and Nageshwar, G.: Excess thermodynamic properties and isobaric vapor-liquid equilibrium data for the binary system: ethyl acetate-2-ethoxy ethanol, J. Inst. Eng. India Chem. Eng. Div., 68, 59-63, 1988.

Tikhonova, N., Timofeev, V., Serafimov, L., and Kupriyanova, Z.: Vapor-liquid equilibrium in the ternary system acetaldehyde Acetone - vinyl acetate at atmospheric pressure, Izv. Vuz Khim. Kh. Tekh., 13, 349-351, 1970.

Tiryaki, A., Gürüz, G., and Orbey, H.: Liquid-liquid equilibria of ternary systems of water + acetone and $\mathrm{C}_{5}$ and $\mathrm{C}_{8}$ alcohols at 298, 303 and 308 K, Fluid Phase Equilibr., 94, 267-280, 1994.

Tochigi, K., Goto, T., Kurita, S., and Kojima, K.: Activity coefficients for water plus phenol system, J. Chem. Eng. Jpn., 30, 1116-1119, doi:10.1252/jcej.30.1116, 1997.

Tsonopoulos, C. and Prausnitz, J. M.: Fugacity coefficients in vapor-phase mixtures of water and carboxylic acids, Chem. Eng. J., 1, 273-278, doi:10.1016/0300-9467(70)85014-6, 1970.
Udovenko, V. V. and Aleksandrova, L.: Vapor pressure in threecomponent systems. IV. Formic acid - benzene - water system, Russ. J. Phys. Ch., 37, 25-27, 1963.

Viala, M.: Etude thermique et cryoscopique des melanges de benzene et dálcool ethylique, B. Soc. Chim. Fr., 15, 5-11, 1914.

Virtanen, A., Joutsensaari, J., Koop, T., Kannosto, J., Yli-Pirilä, P., Leskinen, J., Mäkelä, J., Holopainen, J., Pöschl, U., Kulmala, M., Worsnop, D. R., and Laaksonen, A.: An amorphous solid state of biogenic secondary organic aerosol particles, Nature, 467, 824827, doi:10.1038/nature09455, 2010.

Wang, L., Cheng, Y., Xiao, X., and Li, X.: Liquid-liquid equilibria for the ternary systems acetic acid + water + butyl acetate and acetic acid + water + 2-methyl propyl acetate at $304.15 \mathrm{~K}$, 332.15 K, and 366.15 K, J. Chem. Eng. Data, 52, 1255-1257, doi:10.1021/je6005746, 2007.

Waradzin, W. and Surovy, J.: Equilibrium data of the liquid-vapor systems containing acetone, vinyl acetate, crotonaldehyde, and acetic acid. I. experimental data for isothermal binary systems processed by means of the Wilson Equation, Chem. Zvesti, 29, 783-794, 1975.

Webb, T. and Lindsley, C.: The cryoscopic study of certain aliphatic alcohols, J. Am. Chem. Soc., 56, 874-878, 1934.

Weidlich, U. and Gmehling, J.: A modified UNIFAC model. 1. Prediction of VLE, $h^{E}$, and $\gamma^{\infty}$, Ind. Eng. Chem. Res., 26, 13721381, doi:10.1021/ie00067a018, 1987.

Wen, C. and Tu, C.: Vapor-liquid equilibria for binary and ternary mixtures of ethanol, 2-butanone, and 2, 2, 4-trimethylpentane at 101.3 kPa, Fluid Phase Equilibr., 258, 131-139, 2007.

Williams, R. and Carnahan, D.: Fracture faces and other interfaces as ice nucleation sites, Cryobiology, 27, 479-482, 1990.

Wright, E. and Akhtar, B.: Soluble surface films of short-chain monocarboxylic acids on organic and aqueous substrates, J. Chem. Soc. B, 151-157, doi:10.1039/J29700000151, 1970.

Xie, Q., Wan, H., Han, M., and Guan, G.: Investigation on isobaric vapor-liquid equilibrium for acetic acid + water + methyl ethyl ketone + isopropyl acetate, Fluid Phase Equilibr., 280, 120-128, 2009.

Yan, W. D., Topphoff, M., Rose, C., and Gmehling, J.: Prediction of vapor-liquid equilibria in mixed-solvent electrolyte systems using the group contribution concept, Fluid Phase Equilibr., 162, 97-113, 1999.

Yaws, C. L., Narasimhan, P. K., and Gabbula, C.: Yaws' Handbook of Antoine Coefficients for Vapor Pressure [electronic resource], Knovel, available at: http://app.knovel.com/hotlink/toc/id:kpYHACVPEH/ yaws-handbook-antoine/yaws-handbook-antoine (last access: 18 August 2013), 2005.

Young, F.: D-glucose-water phase diagram, J. Phys. Chem., 61, 616-619, doi:10.1021/j150551a023, 1957.

Zardini, A. A., Sjogren, S., Marcolli, C., Krieger, U. K., Gysel, M., Weingartner, E., Baltensperger, U., and Peter, T.: A combined particle trap/HTDMA hygroscopicity study of mixed inorganic/organic aerosol particles, Atmos. Chem. Phys., 8, 55895601, doi:10.5194/acp-8-5589-2008, 2008.

Zobrist, B., Marcolli, C., Pedernera, D. A., and Koop, T.: Do atmospheric aerosols form glasses?, Atmos. Chem. Phys., 8, 52215244, doi:10.5194/acp-8-5221-2008, 2008. 
Zou, M. and Prausnitz, J.: Vapor-liquid and liquid-liquid equilibria in binary aqueous systems, J. Chem. Eng. Data, 32, 34-37, doi:10.1021/je00047a009, 1987.

Zuend, A. and Seinfeld, J. H.: Modeling the gas-particle partitioning of secondary organic aerosol: the importance of liquidliquid phase separation, Atmos. Chem. Phys., 12, 3857-3882, doi:10.5194/acp-12-3857-2012, 2012.

Zuend, A. and Seinfeld, J. H.: A practical method for the calculation of liquid-liquid equilibria in multicomponent organicwater-electrolyte systems using physicochemical constraints, Fluid Phase Equilibr., 337, 201-213, 2013.

Zuend, A., Marcolli, C., Luo, B. P., and Peter, T.: A thermodynamic model of mixed organic-inorganic aerosols to predict activity coefficients, Atmos. Chem. Phys., 8, 4559-4593, doi:10.5194/acp8-4559-2008, 2008.
Zuend, A., Marcolli, C., Peter, T., and Seinfeld, J. H.: Computation of liquid-liquid equilibria and phase stabilities: implications for RH-dependent gas/particle partitioning of organic-inorganic aerosols, Atmos. Chem. Phys., 10, 7795-7820, doi:10.5194/acp10-7795-2010, 2010.

Zuend, A., Marcolli, C., Booth, A. M., Lienhard, D. M., Soonsin, V., Krieger, U. K., Topping, D. O., McFiggans, G., Peter, T., and Seinfeld, J. H.: New and extended parameterization of the thermodynamic model AIOMFAC: calculation of activity coefficients for organic-inorganic mixtures containing carboxyl, hydroxyl, carbonyl, ether, ester, alkenyl, alkyl, and aromatic functional groups, Atmos. Chem. Phys., 11, 9155-9206, doi:10.5194/acp11-9155-2011, 2011. 